University of Louisville

ThinkIR: The University of Louisville's Institutional Repository

$12-2020$

\title{
Modeling and analysis for unit cell size, material anisotropy and material imperfection effects of cellular structures fabricated by powder bed fusion additive manufacturing.
}

Yan Wu

University of Louisville

Follow this and additional works at: https://ir.library.louisville.edu/etd

Part of the Manufacturing Commons, and the Metallurgy Commons

\section{Recommended Citation}

Wu, Yan, "Modeling and analysis for unit cell size, material anisotropy and material imperfection effects of cellular structures fabricated by powder bed fusion additive manufacturing." (2020). Electronic Theses and Dissertations. Paper 3545.

https://doi.org/10.18297/etd/3545

This Doctoral Dissertation is brought to you for free and open access by ThinkIR: The University of Louisville's Institutional Repository. It has been accepted for inclusion in Electronic Theses and Dissertations by an authorized administrator of ThinkIR: The University of Louisville's Institutional Repository. This title appears here courtesy of the author, who has retained all other copyrights. For more information, please contact thinkir@louisville.edu. 
MODELING AND ANALYSIS FOR UNIT CELL SIZE, MATERIAL ANISOTROPY AND

MATERIAL IMPERFECTION EFFECTS OF CELLULAR STRUCTURES FABRICATED

BY POWDER BED FUSION ADDITIVE MANUFACTURING

\author{
By \\ Yan $\mathrm{Wu}$ \\ B.E., Wuhan University of Technology, 2012 \\ M.E., Huazhong University of Science and Technology, 2015
}

\begin{abstract}
A Dissertation
Submitted to the Faculty of the

J. B. Speed School of Engineering of the University of Louisville in Partial Fulfillment of the Requirements

for the Degree of

Doctor of Philosophy

In Industrial Engineering

Department of Industrial Engineering

University of Louisville

Louisville, Kentucky
\end{abstract}

December 2020 
Copyright 2020 by Yan Wu

All rights reserved 

MODELING AND ANALYSIS FOR UNIT CELL SIZE, MATERIAL ANISOTROPY AND

MATERIAL IMPERFECTION EFFECTS OF CELLULAR STRUCTURES FABRICATED

BY POWDER BED FUSION ADDITIVE MANUFACTURING

By

Yan $\mathrm{Wu}$

B.E., Wuhan University of Technology, 2012

M.E., Huazhong University of Science and Technology, 2015

A Dissertation Approved on

November 27, 2020

by the following Dissertation Committee:

Dr. Li Yang (Chair)

Dr. Kevin Chou

Dr. Thomas Berfield

Dr. Thomas Starr

Dr. Erin Gerber 


\section{DEDICATION}

This dissertation is dedicated to my parents

Mr. Wenqing Wu and Mrs. Xiaoqiong Zhang

And

My wife Mrs. Ying Zhou

for all the love and support. 


\section{ACKNOWLEDGMENTS}

I wish to express my sincere appreciation to my supervisor, Dr. Li Yang, whose expertise was invaluable in formulating the research questions and methodology in the additive manufacturing and light weight design area. His insightful feedback pushed me to sharpen my thinking and brought my work to a higher level. Without his persistent help, the goal of this research would not have been realized.

I want to thank other committee members, Dr. Kevin Chou, Dr. Thomas Starr, Dr. Erin Gerber and Dr. Thomas Berfield, for their advice and support during this project. I have great respect and admiration for each one of you and truly appreciate your participation in this project.

I would also like to take this opportunity to thank all my colleagues and friends, Shanshan Zhang, Hadi Miyanaji, Yuan Zhang, Austin Lassell, Chris O’Neil. It is my great pleasure to have them around. Special thanks to Tim Gornet, Ed Tackett, Gary Graf, Sean Dobson, who help me with various equipment and systems and perform experiments for me.

I wish to acknowledge the support and great love of my family, my wife, Ying; my mother, Xiaoqiong; and my father, Wenqing. They kept me going on and this work would not have been possible without their input. 


\begin{abstract}
MODELING AND ANALYSIS FOR UNIT CELL SIZE, MATERIAL ANISOTROPY AND

MATERIAL IMPERFECTION EFFECTS OF CELLULAR STRUCTURES FABRICATED

BY POWDER BED FUSION ADDITIVE MANUFACTURING
\end{abstract}

Yan $\mathrm{Wu}$

November 27, 2020

Cellular structures are networks of interconnected struts or walls with porosities and are widely found in many natural load-bearing structures such as plants and bones. Cellular structures offer unique functional characteristics such as high stiffness to weight ratio, tailorable heat transfer coefficient, and enhanced mechanical energy absorption, which makes them highly attractive in various engineering disciplines such as biomedical implants, electrodes, heat exchangers, and lightweight structures. There exists an abundance of literatures that have investigated various mechanical properties of various cellular structures such as Poisson's ratio, elastic modulus, ultimate strength, yield strength, and failure characteristics. Based on the classic cellular structure model, these mechanical properties are highly dependent on both the relative density and the topologies of the unit cell designs. Cellular structures with higher relative densities generally exhibit higher overall mechanical properties. In addition, there also exist multiple general design rules for cellular unit cell topology designs, such as nodal connectivity-based deformation mechanism and re-entrant auxetic mechanism. However, currently most theoretical knowledge for cellular structures are established based on infinite pattern sizes, i.e. infinite 
numbers of unit cells along all principal symmetry directions. On the other hand, for the cellular structures with finite sizes that are commonly designed in real-world applications, in addition to relative density and cell topology, the cellular pattern size effects, which are introduced by the non-ideal boundary conditions, also plays important roles in determining the overall mechanical characteristics of the cellular structure. As a result, many equations and conclusions from the classic Ashby and Gibson models cannot be directly applied to these finite-size cellular structures, which significantly limits the designability of cellular structures for various dimension-limited applications. Besides, due to the complex geometry of cellular structures, additive manufacturing (AM) processes have been considered as the only practically viable option for their fabrication, which introduce various manufacturing-related design variables with material properties such as material anisotropy and material imperfection. In order to adequately design for cellular structures realized by AM processes, a modeling approach that enables comprehensive analysis of all these factors are desirable.

In this work, an analytical model framework was established for the analysis of mechanical characteristics of the finite-size cellular structure with imperfect local material properties. The model was verified by the experimental results for both mechanical properties and cellular fracture failure propagation patterns with samples fabricated via powder bed fusion (PBF) process. The results showed that the models could not only provide good predictions to both average mechanical properties and their variabilities, but also adequately capture the effects of the finite pattern size effects and local material heterogeneity effects. Based on the established model, the topology-material-mechanical properties of the finite-size AM cellular structures were investigated in detail. More 
specifically, the effects of pattern size-topology, material anisotropy and the material imperfections were studied systematically. Various new insights were obtained, including:

1. Based on the modeling analysis, the effects of size and topology on the tensile failure behavior of multiple representative cellular structures (2D auxetic, 2D diamond, 2D triangular1 and 2D triangular2) under various geometry design conditions (including cell topology, cell size and number of unit cells) were systematically investigated. It was found that the $2 \mathrm{D}$ bending-dominated structures with lower nodal connectivity (number of struts that meet in joints) (2D auxetic and 2D diamond) exhibited a relatively progressive crack propagation pattern, while the $2 \mathrm{D}$ stretching-dominated structures with higher nodal connectivity (2D triangular1 and 2D triangular2) appear to exhibit rather catastrophic brittle fracture failure. During the failure fracture propagation, the energy absorption of the 2D stretching-dominated structures were significantly higher than that of the 2D bendingdominated structures. Moreover, for all cellular designs, the tensile failure behaviors tend to converge to more consistent patterns when the cellular structure pattern sizes increase beyond certain thresholds that are dependent on the cellular topology designs.

2. The material anisotropy effects, which are characteristic to AM processes, were explored through both analytical modeling analysis and experiments on three representative 3D cellular structures (auxetic, BCC and octahedral). The established models were verified via experimentation with samples fabricated by electron beam PBF (EB-PBF) process using Ti6Al4V as material, using the material anisotropy information established experimentally using single struts with different build orientations $\left(0^{\circ}, 15^{\circ}, 30^{\circ}\right.$, $45^{\circ}, 60^{\circ}, 75^{\circ}$ and $90^{\circ}$ ). The predicted mechanical properties of the Ti6Al4V cellular structures showed good agreement with experimental results. It was shown that both the 
strength and elastic modulus anisotropy of the materials affect the strength of the cellular structures, which must be determined based on the topology design. In addition, the material anisotropy-topology effects on cellular structures of varying cellular pattern sizes were also investigated in order to quantify the pattern size effects. It was also found that the pattern size effects and the material anisotropy effects can be decoupled during the design of the mechanical properties of these cellular structures.

3. The local material property fluctuation caused by the material imperfection is another important factor to consider for adequate design of AM cellular structures. The local material and feature imperfections affect the overall structural properties of cellular structures and are typically unavoidable with the current AM process technologies. Three representative 2D cellular designs including auxetic, diamond and triangular structures were modeled and analyzed based on the established model, which allows for the implementation of heterogeneous material imperfection at full-scale cellular structure level. The material property imperfection was represented by 3 levels of variabilities $(2 \%, 5 \%$ and $10 \%)$ for both elastic modulus and strength, defined at local cellular element level. Experimental verification using Ti6Al4V cellular structures fabricated via laser PBF (LPBF) process demonstrated the potential of the established model in providing accurate predictions to the mechanical property variability of the cellular structures. In addition, the results also revealed new insights into the topology-material imperfection coupling relationships for the cellular structures. 


\section{TABLE OF CONTENTS}

PAGE

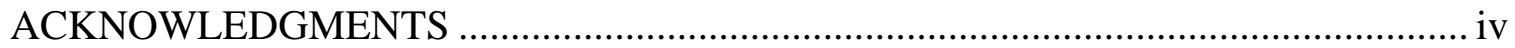

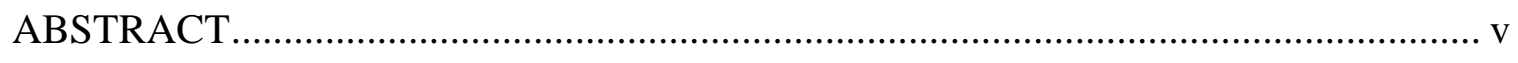

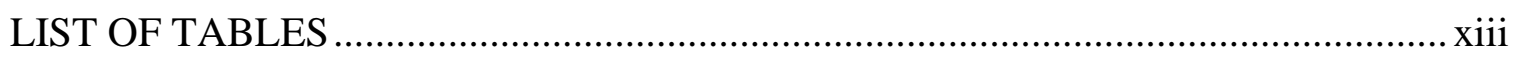

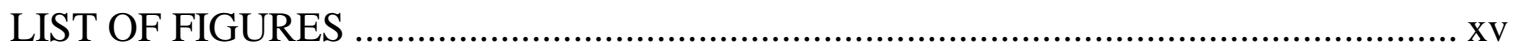

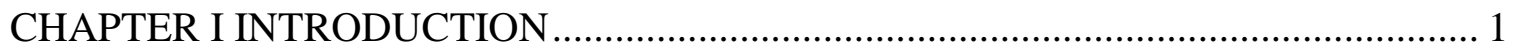

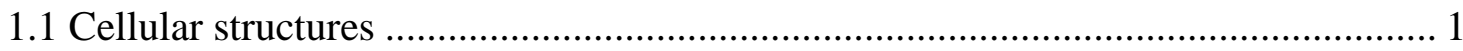

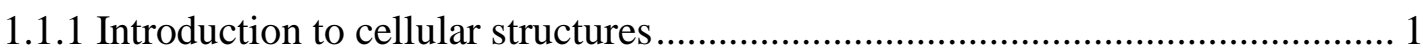

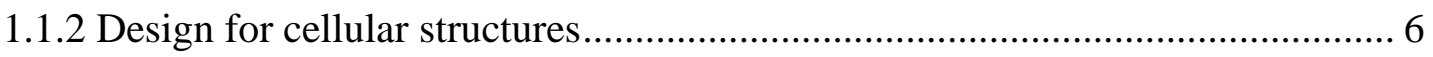

1.1.3 Mechanical properties of cellular structures ................................................... 12

1.2 Modeling and Manufacturing of Cellular Structures ............................................ 19

1.2.1 Modeling approaches of mechanical properties of cellular structures .............. 19

1.2.2 Additive manufacturing of cellular structure ..................................................... 26

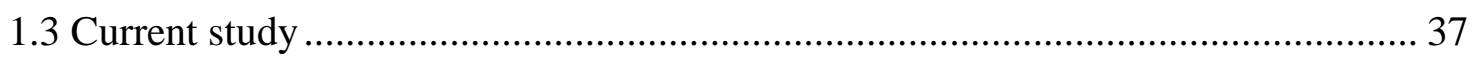

CHAPTER II MODELING AND CHARACTERIZATION OF CELLULAR

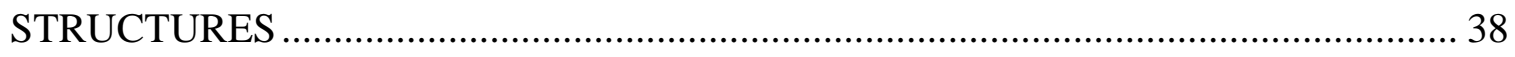

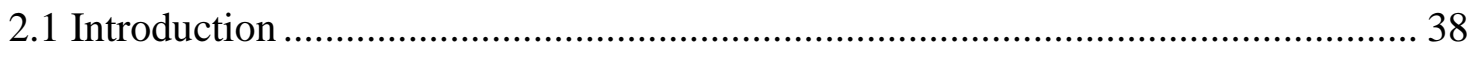


2.2 Analytical modeling of cellular structures

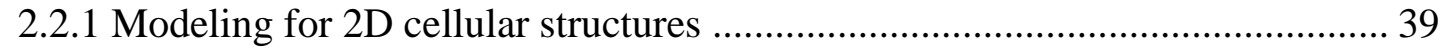

2.2.2 Modeling for 3D cellular structures ....................................................... 44

2.3 Mechanical and failure characteristics of cellular structures ................................54

2.3.1 Mechanical properties of cellular structures ............................................. 54

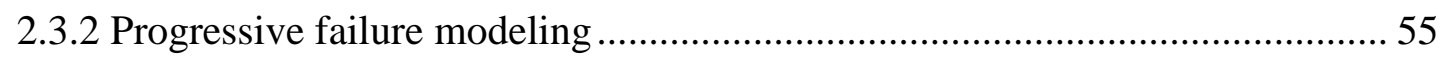

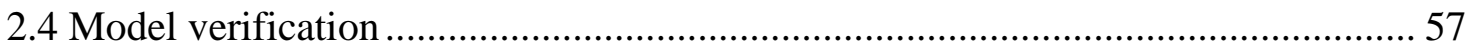

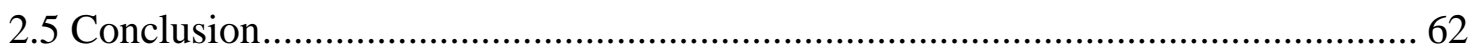

CHAPTER III THE EFFECT OF UNIT CELL SIZE AND TOPOLOGY ON TENSILE

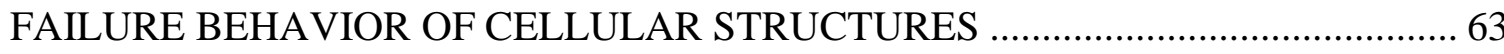

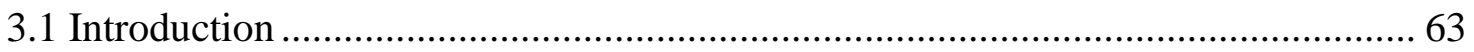

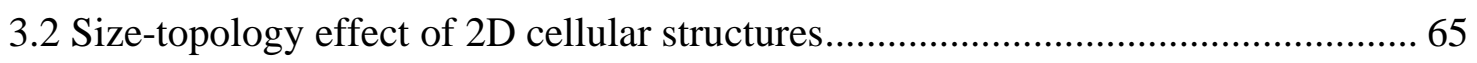

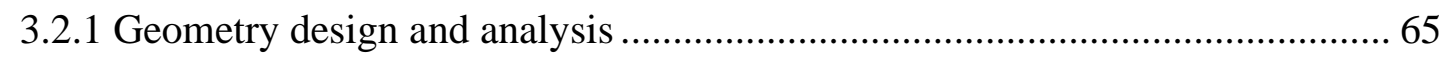

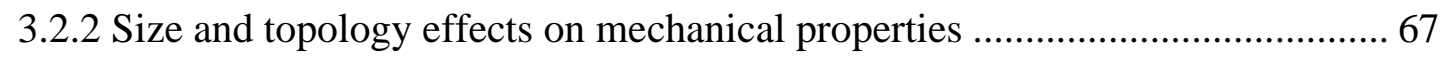

3.2.3 Size and topology effects on tensile failure responses .............................. 76

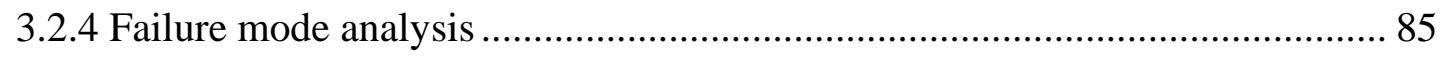

3.3 Size-topology effect of 3D cellular structures.............................................. 90

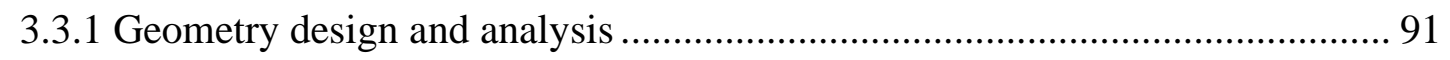

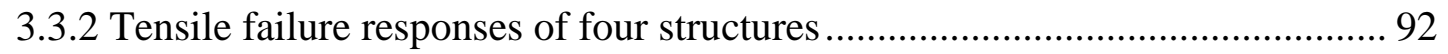


3.3.3 Tensile strength, Young's modulus and energy absorption analysis of four structures 94

3.3.4 Tensile failure pattern of four structures 96

3.4 Conclusions 100

CHAPTER IV MODELING AND ANALYSIS OF MATERIAL ANISOTROPYTOPOLOGY EFFECTS OF 3D CELLULAR STRUCTURES FABRICATED BY POWDER BED FUSION ADDITIVE MANUFACTURING 102

4.1 Introduction 102

4.2 Cellular structure designs 103

4.3 Evaluation of material anisotropy of the strut with different angles 105

4.4 Effect of the material anisotropy-topology on structural mechanical properties .. 109 4.4.1 Effect of the material anisotropy on structural mechanical properties 109

4.4.2 Effect of the pattern size on the anisotropic structural mechanical properties 119 4.5 Experimental verification 128

4.6 Conclusions 131

CHAPTER V MODELING OF THE EFFECT OF LOCAL MATERIAL

IMPERFECTION TO THE STRUCTURAL MECHANICAL PROPERTY

VARIABILITY OF 2D FINITE-SIZE CELLULAR STRUCTURES 133

5.1 Introduction 133

5.2 Modeling for cellular structures with material property variability 136 
5.3 Effect of material property variability on the mechanical properties of cellular structures

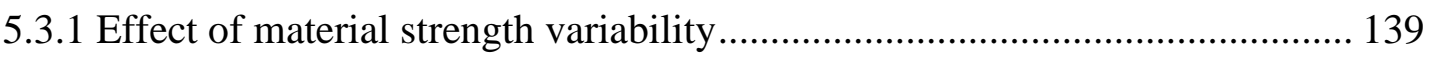

5.3.2 Effect of material modulus variability....................................................... 155

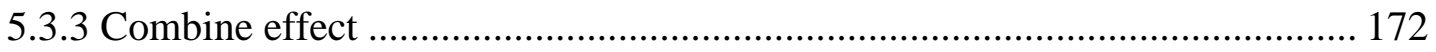

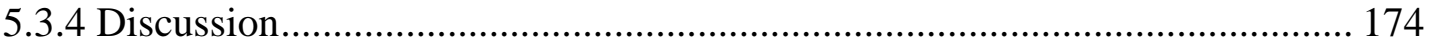

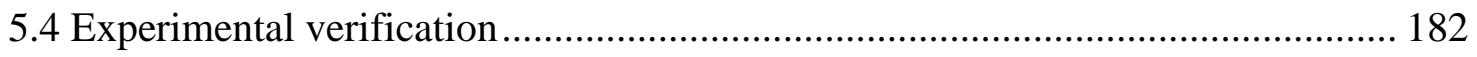

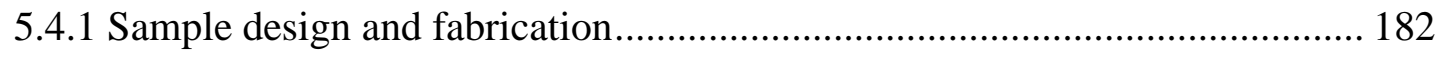

5.4.2 Mechanical properties of cellular structures........................................... 184

5.4.3 Failure response of cellular structures ..................................................... 187

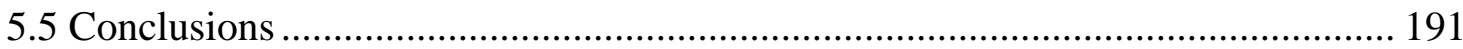

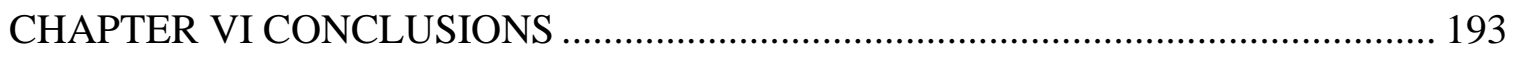

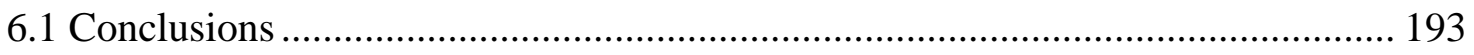

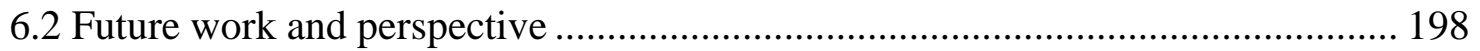

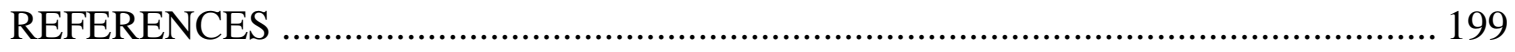

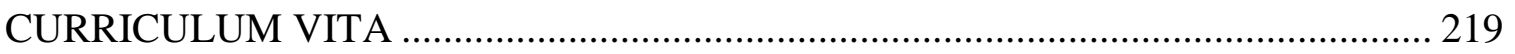




\section{LIST OF TABLES}

Table 1. 1 Tensile properties for Ti6Al4V parts built with directed energy deposition (DED), L-PBF and EB-PBF. 32

Table 2. 1 Comparison of the analytical prediction and the actual experimental results . 60 Table 3. 1 Relative density (RD, \%) under design parameters for different designs ....... 66

Table 3. 2 The Young's modulus and tensile strength versus relative density ................. 73

Table 3. 3 Scaling relationships among the stepwise Young's modulus and tensile strength for two bending-dominated structures 75

Table 4. 1 Experimental mechanical properties of the single struts with different orientations. 108

Table 4. 2 Relative density for different structures with different angles 111

Table 4. 3 Relative density-normalized compressive strength and Young's modulus of cellular designs with different strut orientations under isotropy (I) and anisotropy (A) conditions..... 111

Table 4. 4 Different factors for the strength/modulus anisotropy ratio of BCC structures 115

Table 4. 5 Different factors for the strength/modulus anisotropy ratio of auxetic structures

Table 4. 6 Different factors for the strength/modulus anisotropy ratio of octahedral structures 118

Table 4. 7 Average strength/modulus anisotropy ratio of different structures 122 
Table 4. 8 Design parameters for the different cellular structures .............................. 128

Table 5. 1 Details of the design parameters of each structure .................................... 137

Table 5. 2 Average tensile strength and Young's modulus of auxetic structures with different unit cell numbers and wall strength variabilities at each failure step ............. 146 Table 5. 3 Average tensile strength and Young's modulus of diamond structures with different unit cell numbers and wall strength variabilities at each failure step ............. 148 Table 5. 4 Average tensile strength and Young's modulus of auxetic structures with different unit cell numbers and wall modulus variabilities at each failure step............. 165 Table 5. 5 Average tensile strength and Young's modulus of diamond structures with different unit cell numbers and wall strength variabilities at each failure step ............. 166 Table 5. 6 Tensile strength (MPa) for different structures under different wall strength variability $(\sigma s)$ when considering the first four identical walls................................ 177

Table 5. 7 Design parameters of each fabricated cellular structure ............................. 183 Table 5. 8 Analytical predictions and experimental results of the mechanical properties of

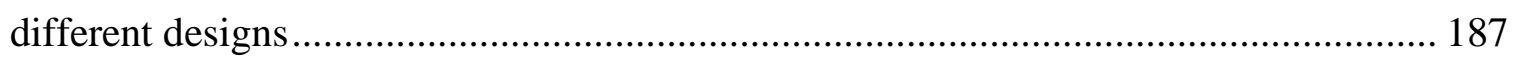




\section{LIST OF FIGURES}

Fig. 1. 1 Examples of several metal foams. The Cymat (a), Alporas (b) and ERG Duocel (c) foams are made from aluminum alloys by particle decomposition (Cymat), gas melt injection (Alporas), and pressure casting (ERG Duocel). The nickel based Incofoam (d) is made by an electroless deposition process. [21] ............................................................ 2

Fig. 1. 2 Five samples of prismatic cellular topologies (a) hexagonal honeycomb used as core material for sandwich panel constructions. (b) triangulated, (c) square, (d)Kagomé, (e)

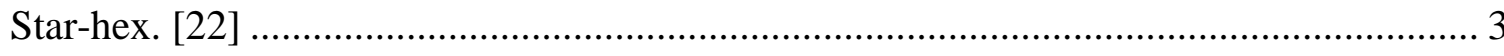

Fig. 1. 3 Cellular truss topologies investigated. All have been made by investment casting. The tetrahedral (b) and pyramidal (d) trusses have also been fabricated by the folding of perforated sheet. In (b), (d) and (e) the cellular truss structure is bounded by solid face

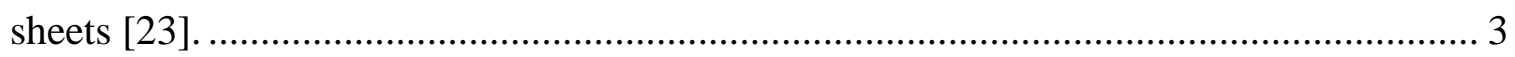

Fig. 1.4 (a) A mechanism; (b) a structure [26] ......................................................... 5

Fig. 1. 5 Schematic of a primitive-based method [28] .................................................. 7

Fig. 1. 6 A unit cell generated using an implicit surface based method [28] .................... 8

Fig. 1. 7 Topology optimization of (a) unit cell and (b) the cellular structures [36] ......... 9

Fig. 1. 8 Octet truss cellular structure using direct patterning method [37] ................... 10

Fig. 1. 9 Uniform cellular structure and conformal cellular structure [38]..................... 11

Fig. 1. 10 Cellular structure obtained by topology optimization method [39] ................ 11

Fig. 1. 11 General compressive behavior of cellular structures during elastic deformation,

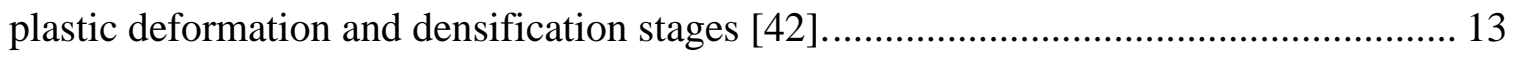


Fig. 1. 12 (a) Schematic drawings of the unit cells used for manufacturing of porous structures; (b) samples specimens from the different types of porous structures.[54] ..... 17

Fig. 1. 13 Deformation of cellular structures under compression: (a) BCC structure [67];

(b) auxetic structure

Fig. 1. 14 Comparison of reported experimental compressive strength (A) and modulus (B) data with predictions of the Gibson-Ashby model [78] ......................................... 23 Fig. 1. 15 (a) Auxetic cellular structure; (b) Loading condition of a unit cell; (c) Loading of the simplified structure [79] 24

Fig. 1. 16 3D tetrahedral elements compared with beam elements: (a) 3D solid mesh using 19,830 elements and $2 \mathrm{~h} 44 \mathrm{~min}$ computational time, (b) one-dimensional beam mesh using 160 elements and $51 \mathrm{~s}$ computational time [86] 26

Fig. 1. 17 Layout of L-PBF system (https://www.additively.com/en/learn-about/lasermelting) 27

Fig. 1. 18 Layout of EB-PBF system (https://www.additively.com/en/learn-about/electronbeam-melting) 28

Fig. 1. 19 Schematic view of the thermal behavior during EB-PBF process for different orientations: vertical (a), oblique (b) and horizontal (c). Purple arrows indicate the thermal flux density and direction [93]. 29

Fig. 1. 20 Comparison of upward and downward-facing surface fabricated by L-PBF [78] 30

Fig. 1. 21 Staircase effect depending on angle [95] 31

Fig. 1. 22 Summary of the tools for the prediction of the effective stiffness of the struts. A designed strut (blue) is produced by the EB-PBF process. The manufactured strut (green) 
is smaller than the designed one. A geometrical (orange) and a numerical (red) equivalent cylinder are extracted to predict the stiffness of the produced strut. [92]........................ 35

Fig. 1. 23 Strut size of the parts made by EB-PBF. [110] ............................................. 35

Fig. 1. 24 Finite element models: (A) numerical models with as-designed geometry, (B) numerical models with distributed geometric imperfections, showing magnified radius variations for each strut, where horizontal struts appear thicker than vertical and diagonal

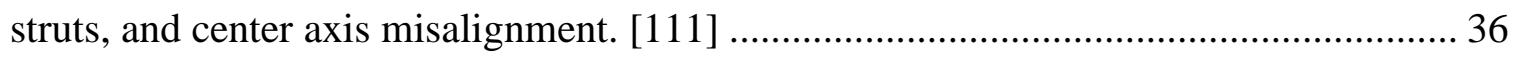

Fig. 2.1 (a)The global coordinate system (xoy) and local coordinate system (xoy) of the 2D beam; (b) A 2D cellular structure with loading conditions. 39

Fig. 2.2 (a) The force equilibrium of the beam; (b) The shear force distributed along the beam; (c) the moment distributed along the beam; (d) The normal stress caused by moment; (e) The normal stress caused by axial force; (f) The shear stress caused by shear force.. 43 Fig. 2. 3 3D Beam element with nodal displacement and nodal loadings 45 Fig. 2. 4 (a)The 3D beam under the global coordinate system; (b) Direction cosines associated with the $x$ axis; (c) Determination local y axis 47 Fig. 2. 5 Special cases of the transformation matrix (a) The local $x$ is in the same direction as the global $\mathrm{z}$; (b) The local $\mathrm{x}$ is in the opposite direction as the global $\mathrm{z}$ 49 Fig. 2. 6 The force components of a 3D strut 49

Fig. 2.7 (a) The normal stress caused by the moment $M y$; (b) The normal stress caused by the moment $M z$; (c) The normal stress caused by axial force $F x$; (d) The shear stress caused by the shear force $F y$; (e)The shear stress caused by the shear force $F z$; (f) The shear stress caused by the torsional moment $M x$; 50

Fig. 2. 8 The traction vectors illustrated on a box element (nine stress components) ...... 52 
Fig. 2. 9 (a) Cellular structure under uniaxial compression loading; (b) loadings on top nodes and bottom nodes .................................................................................. 55

Fig. 2. 10 Demonstration of progressive failure process under tensile loading............... 57

Fig. 2. 11 The detailed design parameters of diamond structure ................................ 57

Fig. 2. 12 Comparisons between experimental and analytical results (strain-stress curve, tensile failure patterns): (a) $2 \times 2$ diamond structure; (b) $3 \times 3$ diamond structure.............. 59

Fig. 2. 13 Displacement and load curves of three random samples ............................. 62

Fig. 3. 1 (a) $3 \mathrm{D}$ view of the $2 \mathrm{D}$ cellular structure; essential parameters of (b) auxetic structure; (c) diamond structure; (d) triangular1 structure; and (e) triangular2 structure. 66 Fig. 3. 2 Stress-strain curves for different cellular structures under different parameters 68 Fig. 3. 3 Tensile strength/ Young's modulus versus design parameters of four types of

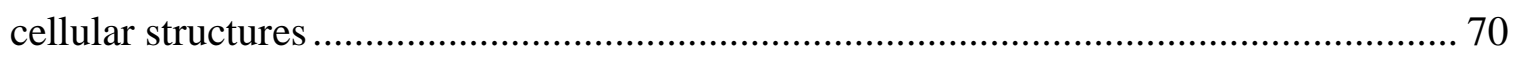

Fig. 3. 4 Averaged stepwise failure characteristics for different design parameters: Stepwise tensile strength (a) and stepwise Young's modulus (b) analysis of 2D auxetic structures; Stepwise tensile strength (c) and stepwise Young's modulus (d) analysis of 2D

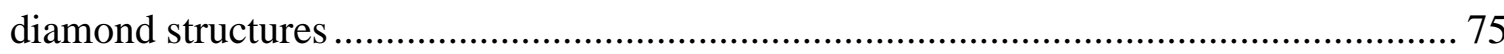

Fig. 3. 5 Energy absorption/ Normalized energy absorption versus design parameters of

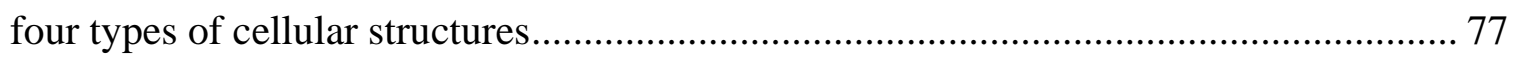

Fig. 3. 6 The tensile failure patterns under different design parameters of $2 \mathrm{D}$ auxetic

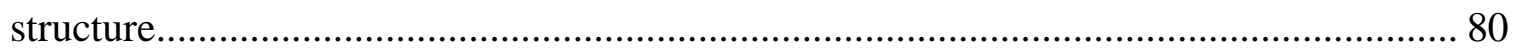
Fig. 3. 7 The tensile failure patterns under different design parameters of 2D diamond structure. 82 
Fig. 3. 8 The tensile failure patterns under different design parameters of 2D triangular1 structure. 83

Fig. 3. 9 The tensile failure patterns under different design parameters of 2D triangular2 structure 84

Fig. 3. 10 The boundary wall A and middle wall B for (a) 2D auxetic, (b) 2D diamond, (c) 2D triangular1 and (d) 2D triangular2 structure and the corresponding ideal structure unit cell: (e) 2D auxetic, (f) 2D diamond, (g) 2D triangular1 and (h) 2D triangular2 86

Fig. 3. 11 The comparison of the normalized principal stresses of wall A and wall B for four structures 88

Fig. 3. 12 Forces and stresses analysis of the of wall $A$ and wall $B$ of $2 \mathrm{D}$ triangular1 structure: (a)Axial force analysis;(b) Shear force analysis;(c) Bending moment analysis; (d) Normal stress analysis; (e) Shear stress analysis 90

Fig. 3.13 (a) BCC; (b) Octahedral; (c) Octet-truss; (d) Auxetic 92

Fig. 3. 14 The strain-stress curves of four structures with different unit cell numbers: (a) BCC; (b) Octahedral; (c) Octet-truss; (d) Auxetic.. 93

Fig. 3. 15 Size effects on the normalized tensile strength (a), normalized Young's modulus (b) and normalized energy absorption (c) of four structures 95

Fig. 3. 16 Fracture patterns of BCC structures with different unit cell numbers 97

Fig. 3. 17 Fracture patterns of octahedral structures with different unit cell numbers ..... 98

Fig. 3. 18 Fracture patterns of octet-truss structures with different unit cell numbers .... 99

Fig. 3. 19 Fracture patterns of auxetic structures with different unit cell numbers 100

Fig. 4. 1 Cellular structures and unit cells: (a) auxetic; (b) BCC; (c) octahedral 104 
Fig. 4. 2 (a) Design and (b)fabrication of the single strut with different angles, and (c) sample surface of struts with different angles produced by EB-PBF process 107

Fig. 4.3 (a) Strain-stress curve of the single struts with different angles; (b) The relationship between the strut angle and relative strength and modulus (normalized by the solid strength and modulus) 108

Fig. 4. 4 Different cellular designs with different strut angles varying from $15^{\circ}$ to $75^{\circ} 110$ Fig. 4. 5 The strength anisotropy ratio of cellular structures under different strut angles 112

Fig. 4. 6 The modulus anisotropy ratio of cellular structures under different strut angles 112

Fig. 4. 7 Different strut types within each type of cellular unit cell (red indicates ones that are subjected to anisotropic material effect, assuming consistent build orientation ( $z$ ). (a) auxetic structure with vertical and inclined struts. (b) BCC structure with inclined struts. (c) octahedral structure with horizontal and inclined struts. 113

Fig. 4. 8 The relationship between relative density-normalized compressive strength/Young's modulus and unit cell numbers of different cellular structures: (a-c) normalized strength of three structures; (d-f) normalized modulus of three structures.. 120 Fig. 4. 9 The stress distribution and structural deformation of the auxetic structures $\left(45^{\circ}\right.$ and $15^{\circ}$ ) with different pattern sizes 124

Fig. 4. 10 The stress distribution and structural deformation of the octahedral structures $\left(45^{\circ}\right.$ and $\left.60^{\circ}\right)$ with different pattern sizes 125

Fig. 4. 11 Failure patterns of the auxetic structures with different pattern sizes for both isotropic and anisotropic materials 126 
Fig. 4. 12 Failure patterns of the octahedral structures with different pattern sizes for both isotropic and anisotropic materials 127

Fig. 4. 13 Fabricated EB-PBF samples 129

Fig. 4. 14 Compressive strength comparison between the predicted strength and experiment strength. 130

Fig. 4. 15 Elastic modulus comparison between the predicted strength and experiment strength. 130

Fig. 5. 1 The designed cellular structures 137

Fig. 5. 2 Strain-stress curves of auxetic structures with different unit cell numbers and wall strength variability:(a,b,c,d) Auxetic 5×5; (e,f,g,h)Auxetic 8×8; (i,j,k,1)Auxetic 10×10 140 Fig. 5. 3 Strain-stress curves of diamond structures with different unit cell numbers and wall strength variability:(a,b,c,d) Diamond 5×5; (e,f,g,h) Diamond 8×8; (i,j,k,l) Diamond $10 \times 10$ 141

Fig. 5. 4 Strain-stress curves of triangular structures with different unit cell numbers and wall strength variability:(a,b,c,d) Triangular $5 \times 5$; (e,f,g,h) Triangular $8 \times 8$; (i,j,k,l) Triangular $10 \times 10$ 142

Fig. 5. 5 Tensile strength calculation results for different designs with material strength variability . 143

Fig. 5. 6 The relationships between the material strength variability and average (a, b c) / standard deviation $(\mathrm{d}, \mathrm{e}, \mathrm{f}))$ of the tensile strengths of the structures for different designs 144

Fig. 5. 7 Energy absorption variability of different designs 150 
Fig. 5. 8 The relationships between the wall strength variability levels and mean $(\mathrm{a}, \mathrm{b} \mathrm{c})$ / standard deviation $(\mathrm{d}, \mathrm{e}, \mathrm{f})$ of cellular energy absorption of different designs 151

Fig. 5. 9 The failure patterns of the auxetic structures with different unit cell numbers and wall strength variabilities 152

Fig. 5. 10 The failure patterns of the diamond structures with different unit cell numbers and wall strength variabilities 153

Fig. 5. 11 The failure patterns of the triangular structures with different unit cell numbers and wall strength variabilities 155

Fig. 5. 12 Strain-stress curves of auxetic structures with different unit cell numbers and wall modulus variabilities:(a,b,c,d) Auxetic 5×5; (e,f,g,h)Auxetic 8×8; (i,j,k,l)Auxetic $10 \times 10$ 157

Fig. 5. 13 Strain-stress curves of auxetic structures with different unit cell numbers and wall modulus variabilities:(a,b,c,d) Diamond 5×5; (e,f,g,h) Diamond 8×8; (i,j,k,l) Diamond $10 \times 10$ 158

Fig. 5. 14 Strain-stress curves of auxetic structures with different unit cell numbers and wall modulus variabilities:(a,b,c,d) Triangular 5×5; (e,f,g,h) Triangular $8 \times 8$; (i,j,k,l) Triangular $10 \times 10$ 159

Fig. 5. 15 Tensile strength calculation results for different designs with material modulus variability 160

Fig. 5. 16 Elastic modulus calculation results for different designs with material modulus variability 161

Fig. 5. 17 The relationships between the material modulus variability and mean (a, b, c) / standard deviation $(\mathrm{d}, \mathrm{e}, \mathrm{f})$ ) of the structural tensile strengths for different designs 163 
Fig. 5. 18 The relationships between the material elastic modulus variability and mean (a, b, c) / standard deviation (d, e, f)) of the elastic modulus for different designs 164

Fig. 5. 19 Energy absorption distribution of different designs 168

Fig. 5. 20 The relationships between the wall strength variability levels and mean (a, b c) / standard deviation ( $d, e, f)$ of cellular energy absorption of different designs 169

Fig. 5. 21 The failure patterns of the auxetic structures with different unit cell numbers and wall modulus variability 170

Fig. 5. 22 The failure patterns of the diamond structures with different unit cell numbers and wall modulus variability. 171

Fig. 5. 23 The failure patterns of the triangular structures with different unit cell numbers and wall modulus variability. 172

Fig. 5. 24 The averaged mechanical properties of different cellular structures with wall strength and elastic modulus variabilities 173

Fig. 5. 25 Relationships between wall strength variability, wall modulus variability and tensile strength for different designs 174

Fig. 5. 26 Probability density curves of tensile strength under different wall strength variability $(\sigma s)$ for different structures considering the first four identical walls 176 Fig. 5. 27 (a)The symetric conditon of cellular structure; (b,c,d) stress levels of top seven critial walls and the correponding locations of difference structures 178 Fig. 5. 28 Probability density curves of critical nominal stress of Wall\#1 and Wall \#2 under different wall strength variability $(\sigma s)$ for different structures 179 Fig. 5. 29 The relationship between the included criticality indices and the average strength reduction 181 
Fig. 5. 30 The failure initiation distribution of different cellular types under different wall strength variability levels: (a,b,c) auxetic structures; (d,e,f) diamond structures; (g,h,i)

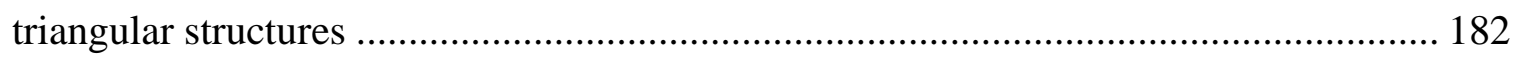
Fig. 5. 31 Experimental verification using Ti6Al4V samples fabricated via EOSINT M270: (a). Fabricated samples; (b). Stress-strain curves from tensile coupons ........................ 184 Fig. 5. 32 Strain-stress curves of (a)auxetic structures, (b) diamond structure; and (c)

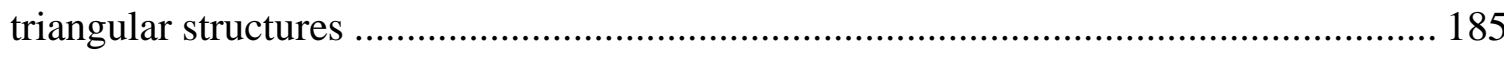

Fig. 5. 33 Strain-stress curves of (a)auxetic structures, (b) diamond structure; and (c)

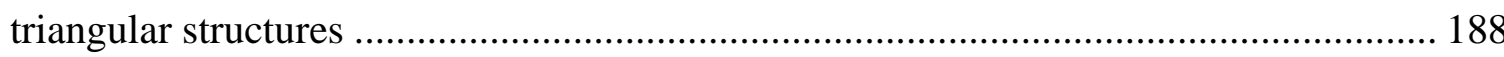

Fig. 5. 34 Boundary conditions of different structures: (a) auxetic structure; (b) diamond

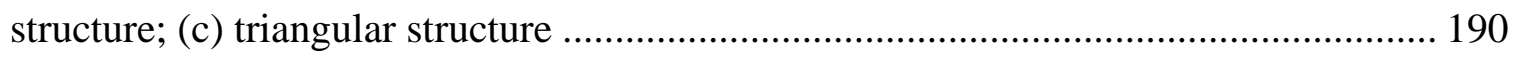
Fig. 5. 35 Simulation results for the perfect (a) auxetic, (b) diamond and (c) triangular

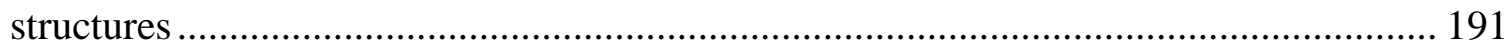




\section{CHAPTER I}

\section{INTRODUCTION}

\subsection{Cellular structures}

\subsubsection{Introduction to cellular structures}

Cellular structures, which are often defined as networks of interconnected struts or walls, exhibit many attractive combinations of mechanical and physical properties that are exploited in engineering designs such as structural sandwich panels[1][2][3][4][5][6][7], impact energy absorbers and protective structures [8][9][10], thermal insulators [11][12], catalyst and biomedical structures $[13][14][15][16][17][18][19]$.

Depending on their topological regularity, cellular structures can be categorized into stochastic and non-stochastic periodic cellular structures [20]. The stochastic cellular structures usually have a random distribution of open or closed voids [21]. Fig. 1.1 shows some examples of stochastic cellular structures. For the Non-stochastic periodic structures, they typically exhibit 2D or 3D topological periodicity (i.e. repeating and ordered unit cells). 2D cellular structures are usually constructed by interconnected thin walls. Fig. 1.2 shows some common 2D cellular structures. Among these examples is the honeycomb structure, which is well known for its efficient 2D load bearing capability, but is not optimal for many loading conditions, as are the cases for most other prismatic-type 2D cellular designs. In comparison, 3D periodic cellular structures (Fig. 1.3) are constructed by either interconnected walls or struts with geometrical periodicity that can usually be represented 
by 3D unit cells. 3D cellular structure designs are generally seen as having superior design abilities for multi-functionality and multi-objective designs compared to stochastic and 2D prismatic-type cellular structures. Various literature has demonstrated the design of 3D periodic cellular structures for various mechanical performance objectives such energy absorption, ultimate strength, elastic modulus and controlled buckling, as well as other objectives such as specific surface area and heat transfer coefficient.
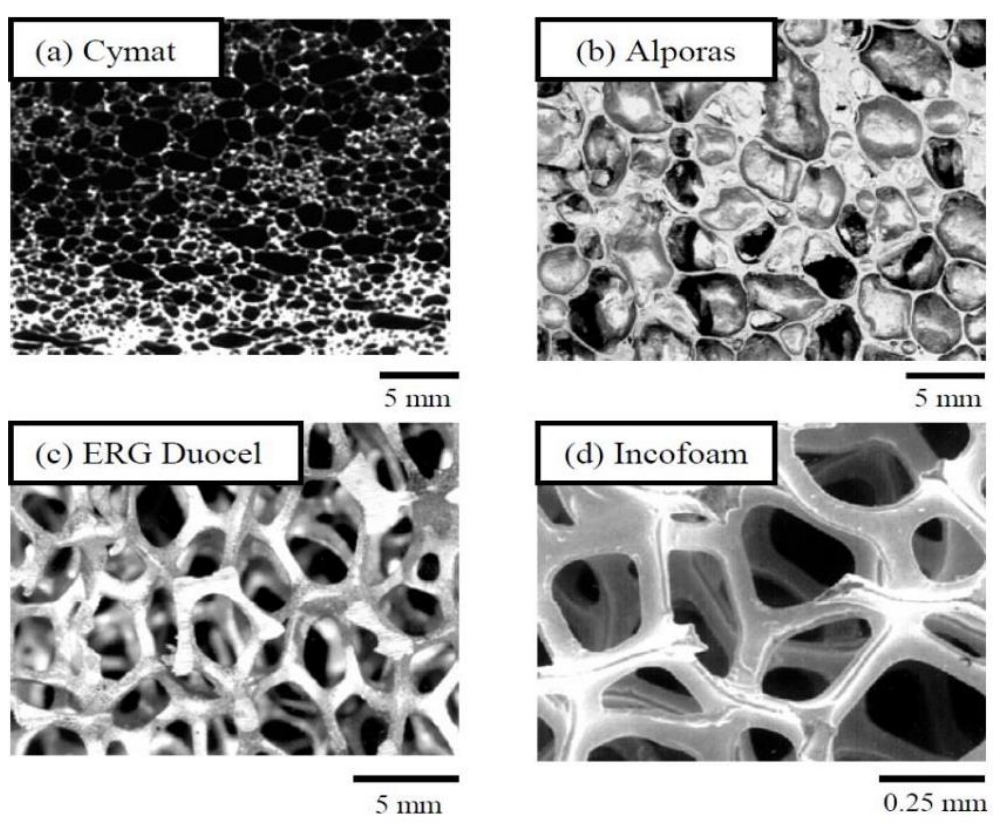

Fig. 1. 1 Examples of several metal foams. The Cymat (a), Alporas (b) and ERG Duocel (c) foams are made from aluminum alloys by particle decomposition (Cymat), gas melt injection (Alporas), and pressure casting (ERG Duocel). The nickel based Incofoam (d) is made by an electroless deposition process. [21] 


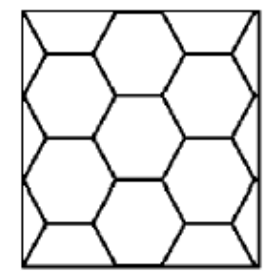

(a)

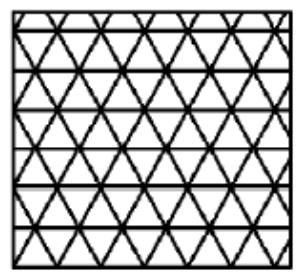

(b)

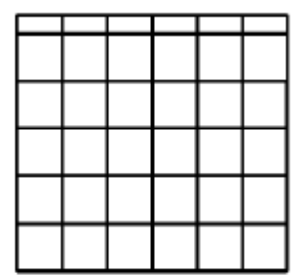

(c)

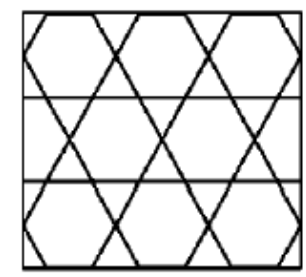

(d)

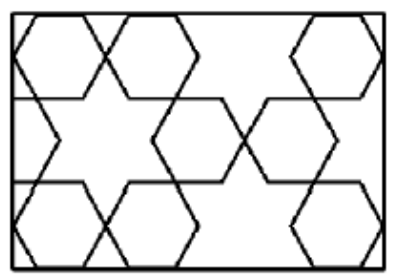

(e)

Fig. 1.2 Five samples of prismatic cellular topologies (a) hexagonal honeycomb used as core material for sandwich panel constructions. (b) triangulated, (c) square, (d)Kagomé, (e) Star-hex. [22]

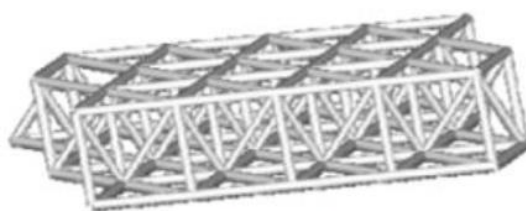

(a) OCTET TRUSS

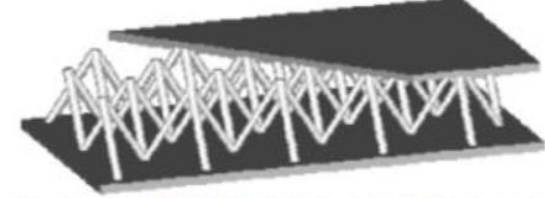

(b) TETRAHEDRAL LATTICE TRUSS

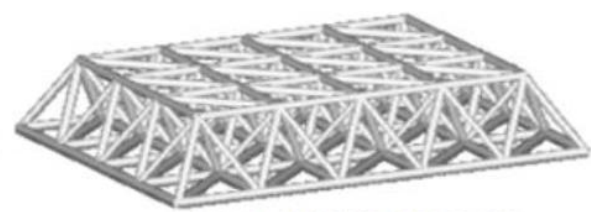

(c) LATTICE BLOCK

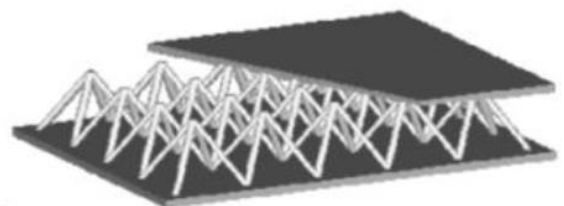

(d) PYRAMIDAL LATTICE TRUSS

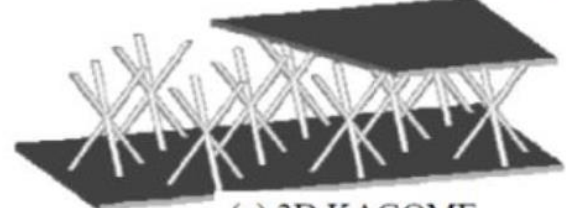

(e) 3D KAGOME

Fig. 1.3 Cellular truss topologies investigated. All have been made by investment casting. The tetrahedral (b) and pyramidal (d) trusses have also been fabricated by the folding of perforated sheet. In (b), (d) and (e) the cellular truss structure is bounded by solid face sheets [23]. 
Apart from stochastic and non-stochastic classification, cellular structures could also be categorized by design rules. For example, based on Maxwell's criterion, the cellular structures can also be either stretching-dominated or bending-dominated. These characteristics can significantly affect its elastic modulus and compliance, and therefore can facilitate the cellular structure design selection. For example, Gibson and Ashby demonstrated that the strength of metal and polymer foams scales as $\bar{\rho}^{1.5}$ when the cell (microstructure) walls are governed by bending [24]. $\bar{\rho}$ is the relative material density of foams compared with solid material. However, the strength of foams scales as $\bar{\rho}$ when their cell walls are governed by stretching. At low relative density levels, e.g. $\bar{\rho}=0.1$, a stretching-dominated structure is about three times as strong as a bending-dominated structure [25].

In order to determine whether a cellular structure is stretching-dominated or bending-dominated, the cellular structures will be treated as a connected set of pin-jointed struts by the following argument. Consider the pin-jointed frames shown in Fig. 1.4. The frame in Fig. 1.4(a) is a mechanism. When loaded, the struts rotate about the joints and the frame collapses; it has neither stiffness nor strength. The triangulated frame shown in Fig. 1.4(b) is a structure: when loaded the struts support axial loads, tensile in some, compressive in others. Thus, the deformation is stretching-dominated and the frame collapses by stretching of the struts. Suppose now that the joints of both frames are "frozen" to prevent free rotation of the struts. On loading the frame shown in Fig. 1.4(a), the struts can no longer rotate. The applied load induces bending moments at the frozen joints, and these cause the struts to bend. This represents the characteristics with most foam structures. However, "freezing" the joints of the triangulated structure has little effect on its 
macroscopic stiffness or strength; although the struts exhibit certain bending, the frame is still stretching-dominated, and the collapse load is dictated mainly by the axial strength of the struts [26].

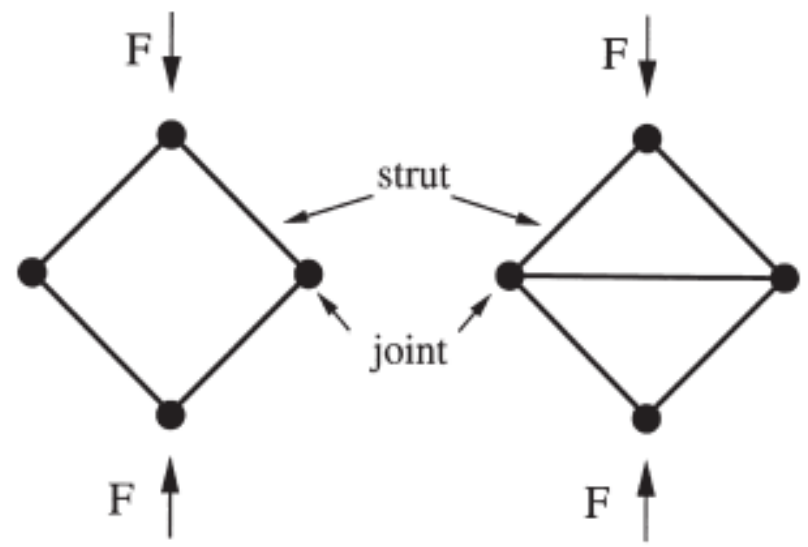

(a)

Fig. 1. 4 (a) A mechanism; (b) a structure [26]

To decide whether a cellular structure is a stretching-dominated structure and bending-dominated structure, Maxwell's criterion [27] is employed. Maxwell suggested an algebraic rule setting out the condition for a pin-jointed frame of $b$ struts and $\mathrm{j}$ frictionless joints to be both statically and kinematically determinate i.e. to be rigid. For 2D and 3D frames, the criteria are:

$$
\begin{aligned}
& 2 D: b=2 j-3 \\
& 3 D: b=3 j-6
\end{aligned}
$$

Using Maxwell's criterion, Deshpande et al. [26] derived that a sufficient condition for the deformation of a periodic structure to be stretching-dominated is that its unit cell (microstructure) consisting of $\mathrm{b}$ struts and $\mathrm{j}$ frictionless joints satisfies Maxwell's criterion for static determinacy shown in Equation (1.3) and Equation (1.4)[25].

2-D lightweight structure: $b-2 j+3 \geq 0$ 
3-D lightweight structure: $b-3 j+6 \geq 0$

Removing struts can transform a stretching-dominated structure into a bendingdominated structure. If a cellular structure satisfies Equation (1.5) or Equation (1.6), the structure will be a bending-dominated structure.

2-D lightweight structure: $b-2 j+3<0$

3-D lightweight structure: $b-3 j+6<0$

Equation (1.3-1.6) provide a design guideline for the selection of cellular structures based on the number of joints and nodes. However, for the more detailed mechanical properties such as tensile/compressive strength, Young's modulus and energy absorption, more specific models are still need.

\subsubsection{Design for cellular structures}

Different types of cellular structures have different and unique characteristics, which can reflect and determine the performance of the whole cellular structures. Therefore, understanding the design of cellular structures are the basic research elements. In order for a cellular structure to be fully defined, the unit cell must be fully characterized in terms of fully describing the structure design and the method of generation. Therefore, the design of a cellular structure includes both unit cell design and pattern design.

\subsubsection{Unit cell design}

A unit cell is the smallest element that make up and characterize the whole cellular structure. It can be designed by using the following three methods: primitive based method, implicit surface based method and topology optimization method [28].

The primitive based method [29][30][31] is a straightforward approach relying on Boolean operations of simple geometric primitives. As shown in Fig. 1.5, the cubic unit 
cell (Fig. 1.5 (b)) is created by Boolean subtraction (Fig. 1.5 (a)) using a cube as the base object and a concentric sphere as the subtractor. The truss-like unit cell (Fig. 1.5 (d)) is created firstly by taking Boolean union of four diagonal oriented cylinders and then by taking Boolean intersection with a cube (Fig. 1.5 (c)).

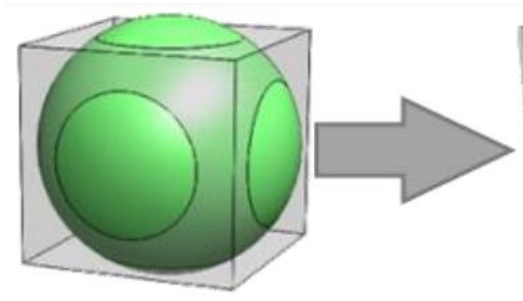

(a)

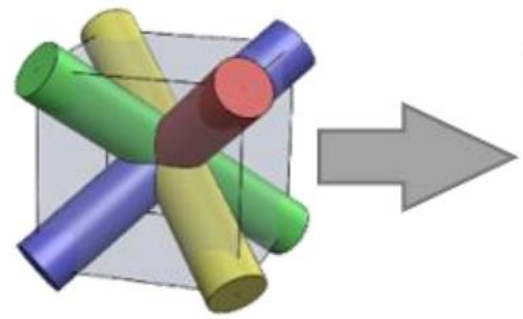

(c)

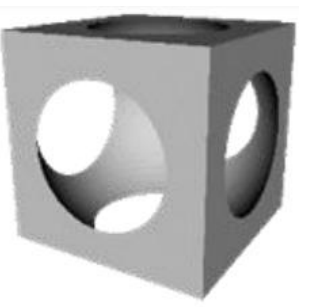

(b)

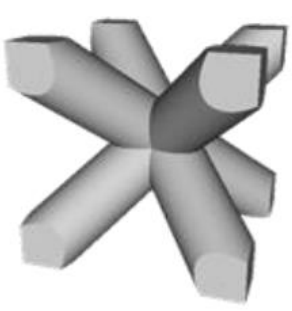

(d)

Fig. 1. 5 Schematic of a primitive-based method [28]

The implicit surface based method [32][33][34][35], in which the surface of the unit cell is defined by mathematical equations, is also an effective approach in the unit cell design. This method uses implicit equations to represent the surface of a unit cell in 3D space. Equation $F(x, y, z)=0$ defines a set of zeros of a function of three coordinates, which determines an array of points that are located on the surface. For example, Fig. 1.6 illustrates a unit cell architecture and its corresponding equation. The porosity of a unit cell refers to the volume fraction of the pores in a unit cell, which significantly influences on the overall mechanical properties. When using a primitive-based method to create the unit cell, porosity is correlated with the dimensions of the primitives, which is sometimes 
inconvenient to adjust. In contrast, a highlighted flexibility enabled by the implicit surface based method is such that the porosity can be parametrically controlled by specifying different terms in the equation.

$$
\begin{aligned}
& \mathrm{F}(x, y, z) \\
& =\cos (2 \pi x)+\cos (2 \pi y)+\cos (2 \pi z) \\
& +a(\cos (2 \pi x) \cos (2 \pi y) \\
& +\cos (2 \pi y) \cos (2 \pi z) \\
& +\cos (2 \pi z) \cos (2 \pi x))+b=0
\end{aligned}
$$

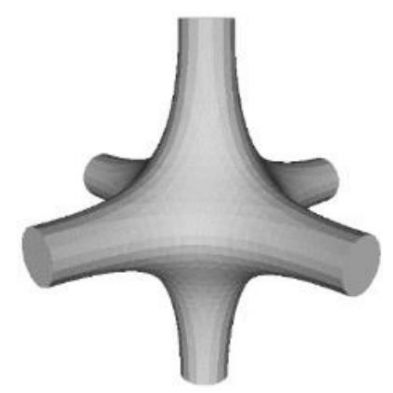

Fig. 1.6 A unit cell generated using an implicit surface based method [28]

The topology optimization method is a mathematical method that optimizes material layout within a given design space, for a given set of loads, boundary conditions and constraints with the goal of maximizing the performance of the system. Topology optimization method are widely used to obtain the cellular structures. By optimizing the design parameters and geometries of the unit cells, topology optimization effectively obtains cellular structures with specific performance. Fig. 1.7 shows an example of topology optimization for generating a unit cell [36]. First, the constraint load was applied to the structure within the design domain and then the topology structure of unit cells under that constraint conditions was optimized. Finally, the cellular structures were formed shown in Fig. 1.7. 


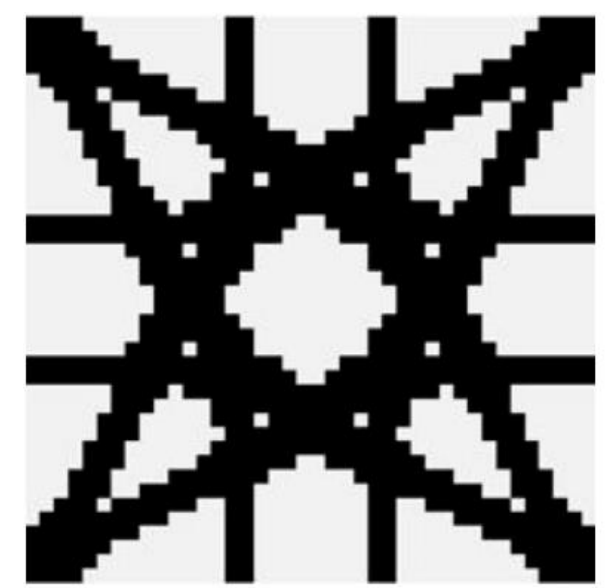

(a)

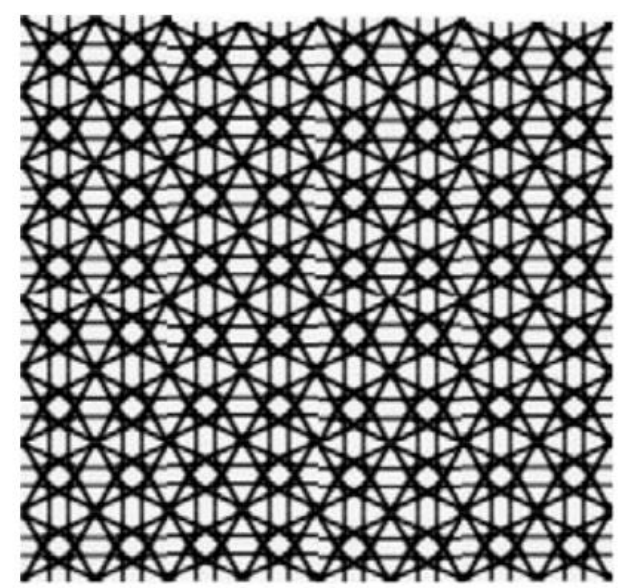

(b)

Fig. 1.7 Topology optimization of (a) unit cell and (b) the cellular structures [36]

\subsubsection{Pattern design}

Once the unit cell type is chosen, the pattern design can further determine the mechanical properties of the cellular structures. Pattern design refers to the way in which the unit cells are repeated in the 3D space. Generally, there are three ways that a unit cell can be arranged in space: direct patterning, conformal patterning, and topology optimization.

For the direct patterning method, the cellular structures are generated by repeating the unit cell in three dimensions along $x, y$, and $z$ axis. For example, Fig. 1.8 shows a $4 \times 4 \times 4$ octet truss cellular structure using the direct patterning method. There are four unit cells in each direction. The direct patterning method can be used for the unit cell created by primitive based method, implicit surface based method and topology optimization method. 


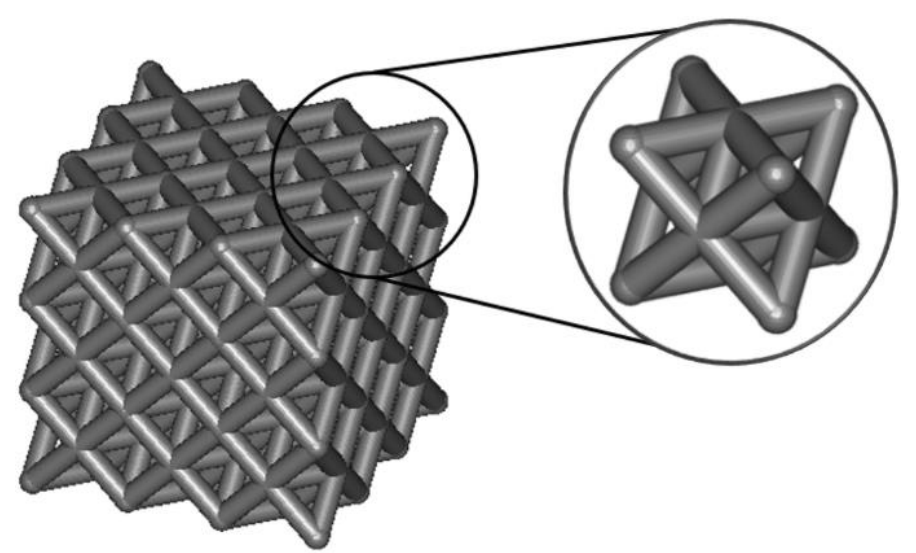

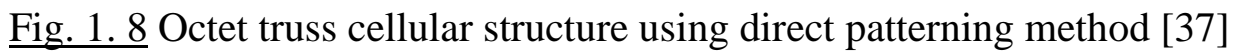

For the conformal patterning method, the unit cells follow a certain surface or volume to obtain the required mechanical properties. The number of unit cells can also be conformed to fit the shape of a design space. Fig. 1.9 shows a comparison between the cellular structures generated by both direct patterning and conformal patterning method. When using the direct patterning method (shown in the left two pictures in Fig. 1.9), the boundary of the design space has numerous unconnected struts, which might decrease the mechanical properties of the design. Besides, these unconnected struts might increase the difficulty for the predictions of its mechanical properties. When the conformal patterning method is adopted, the number and shape of the unit cell can be adjusted to best fit the design space (shown in the right two pictures in Fig. 1.9). 


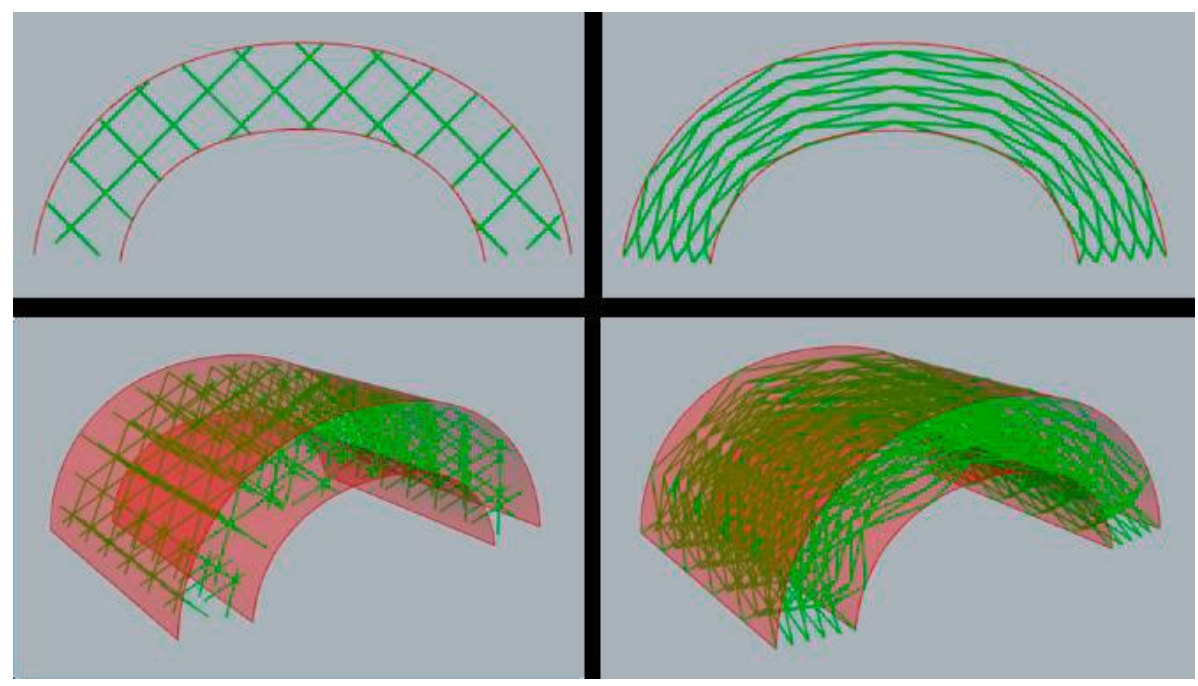

Fig. 1.9 Uniform cellular structure and conformal cellular structure [38]

Using the topology optimization method, the number and size of the unit cell can also be optimized to achieve better cellular performance. Generally, the distribution of the unit cells is guided based on the stress field characteristics under applied loadings. Fig. 1.10 shows an example of the cellular structures generated by the topology optimization method. Unit cells with different porosities are utilized as material voxels to replace the intermediate density obtained from the unpenalized Solid Isotropic Material with Penalization (SIMP) approach to achieve better performance and structural continuity.

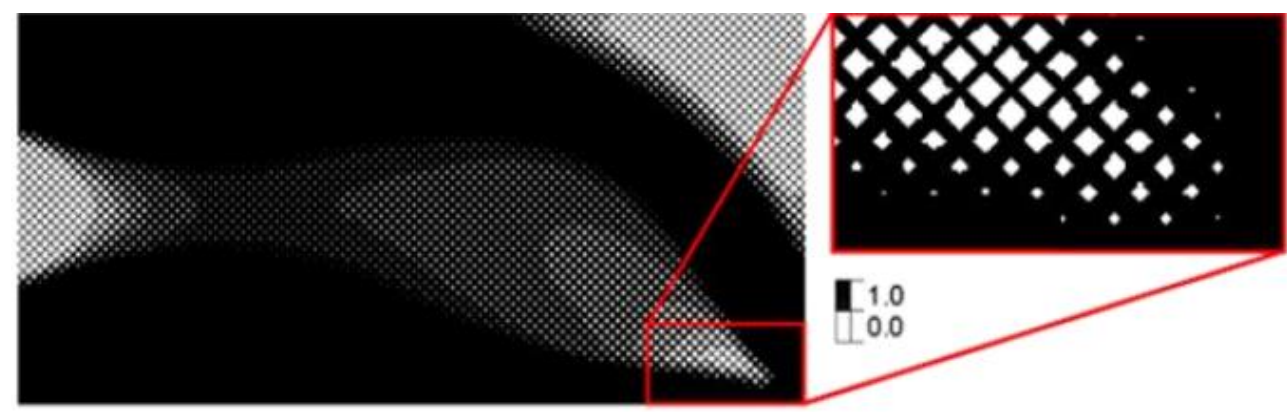

Fig. 1.10 Cellular structure obtained by topology optimization method [39] 


\subsubsection{Mechanical properties of cellular structures}

Similar to the continuous bulk materials [40], most commonly used terms are also applied to cellular structures such as tensile/compressive strength, Young's modulus, Poison's ratio and energy absorption. It was generally recognized that these mechanical properties are highly dependent on both the relative density and the topologies of the unit cell designs. Relative density, which is defined as the ratio between the space occupied by the solid materials and the geometric bounding volume (GBV), usually influences the mechanical properties of the cellular structures following the classic Gibson-Ashby relationships [40]. Cellular structures with higher relative densities generally exhibit higher overall mechanical properties. On the other hand, the unit cell topology-property relationships are more complicated, although there also exist some design rules in the literature. For example, based on the joint connectivity, cellular structures can be categorized into stretch-dominated and bending-dominated types [41][26], which is discussed in Section 1.1.1. In general, the elastic modulus and strength of a stretchingdominated cellular structure scales linearly as a function of the relative density, as opposed to those of a bending-dominated structure that scale exponentially with the relative density. In addition, the toughness of a bending-dominated structure is expected to be larger than that of a stretching-dominated structure with similar relative density.

\subsubsection{Compressive characteristics}

The general deformation behavior of cellular structures can be divided into three discreet stages (Fig. 1.11): linear elastic deformation; plastic deformation and densification [42]. During elastic deformation, the material response is linear elastic with a modulus proportional to the structure material's elastic modulus. Once the elastic limit is reached, 
plastic deformation begins as cells begin to yield, buckle or fracture. For bendingdominated structures deformation continues with a generally constant stress, referred to as the plateau stress, whereas the stress required for further deformation might oscillate in stretch-dominated structures. Once cell components deform enough that contact with other components occurs, constraining further deformation, the densification strain is reached, and densification begins as stress steeply increases.

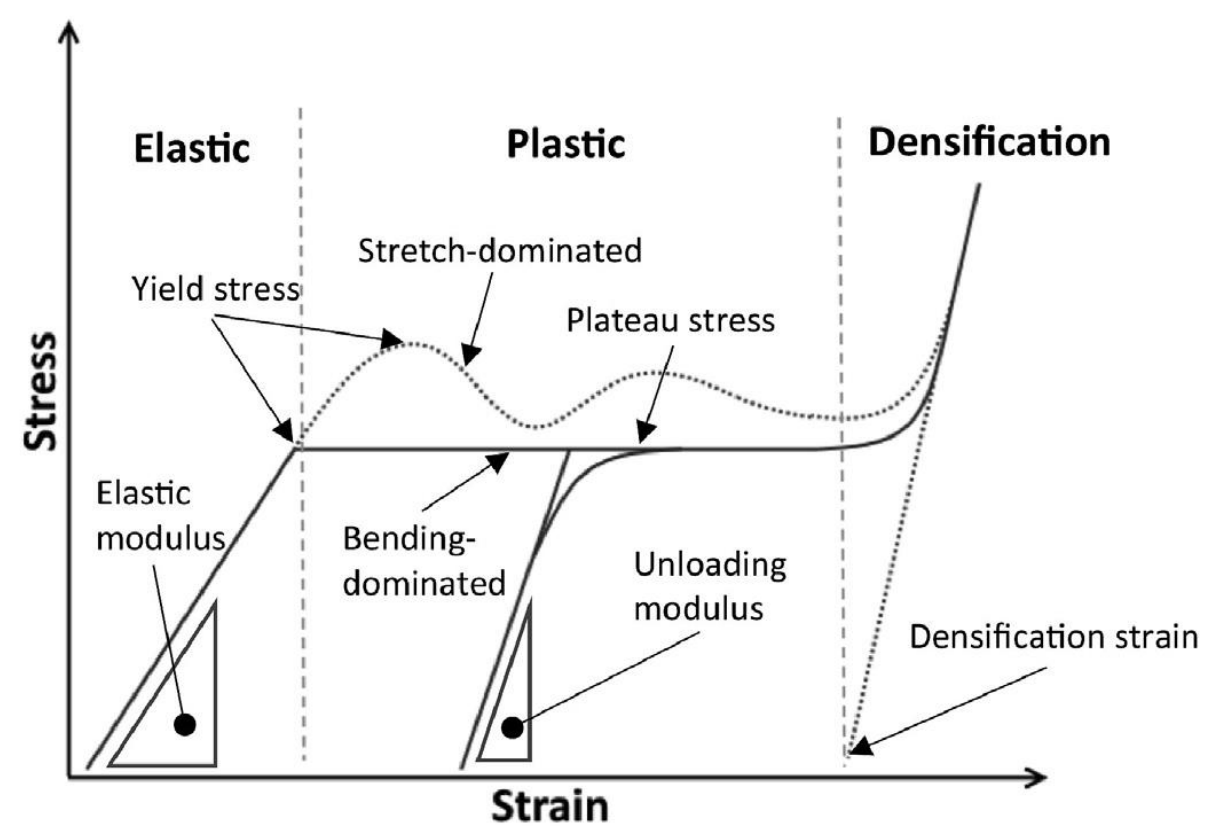

Fig. 1.11 General compressive behavior of cellular structures during elastic deformation, plastic deformation and densification stages [42].

Considering the large design space of the cellular structures, the general compressive deformation behaviors discussed above are not always representative and accurate for all the cellular designs. The type of unit cells, unit cell geometries, materials, process parameters and heat treatments also play an important role in determining the compressive performance of cellular structures. 
1. Unit cell topology: Numerous studies have been conducted to investigate the compressive performance of cellular structures with different unit cell topologies such as face-centered cubic [43], rhombic dodecahedron [44][45], cubic [46][47] and diamond [48][49]. Shi, X. et al. [50] investigated the novel triply periodic minimal surface cellular structures with four unit cell types (gyroid, diamond and primitive) through experiments laser PBF (L-PBF) process and simulations. It was found that gyroid and diamond structures all exhibit shear failure and bending-torsion coupled dominated deformation. The deformation mode of primitive structures is stretch-dominated and characterized by layer-by-layer collapse. Gyroid structures exhibit the high energy absorption among the four triply periodic minimal surface (TPMS) cellular structures due to the high densification strain and plateau stress. Liu, X. et al. [51] explored the geometrical features dominating deformation behaviors and their associated compressive properties of three different AlSi10Mg cellular structures fabricated by laser powder bed fusion. The truncated octahedron- unit cell (TO) cellular structures exhibited highest stiffness and plateau stress among the studied cellular structures. The body centered cubic-unit cell (BCC) and TO cellular structures experienced the formation of shear bands with stress drops, while the hexagon-unit cell (Hexa) cellular structure behaved in a continuous deformation and flat plateau region. The Hexa cellular structure densified at a smaller strain than the BCC and TO cellular structures, due to high density of the struts in the compressive direction. Static and high-speed indentation tests revealed that the TO and Hexa exhibited small strain rate dependence of the compressive strength, whereas the BCC structure showed significant strain rate dependence. Among the cellular structures in their study, the TO structure exhibited the highest energy absorption capacity comparable to previously reported cellular 
structures. Even for the cellular structures with same unit cells, local design modifications may also lead to a different compressive property. Polyamide BCC cellular structure with reinforcing strut demonstrated superior modulus and plateau strength than those without reinforcing strut but possessed significant mechanical anisotropy with a change in the loading direction. The same structure designed with graded density exhibit different deformation behavior. BCC cellular structure with graded density had been found to have distinct deformation mechanism as compared to the design with uniform density [52].

2. Materials type: The effect of intrinsic material properties on the properties of the cellular structures is generally straightforward. Materials with higher strength and modulus usually correspond to have higher strength and modulus with the cellular structures. For the cellular structures with brittle materials, the plastic deformation stage is always shorter compared with that of the same structures with ductile materials. On the other hand, with AM cellular structures the behaviors of materials might deviate from those of the traditionally made ones. Merkt, S. et al. [43] investigated the compressive properties of cellular structure with different materials (TiAl6V4 and 316L). It was found that TiAl6V4 cellular structures show brittle behavior and low energy absorption capabilities compared to the more ductile-behaving $316 \mathrm{~L}$ cellular structures.

\subsubsection{Fatigue performance}

The fatigue performance of cellular structures is critical for many high-value technical applications such as biomedical implant and aerospace components which are subject to stringent limits associated with cyclic loading [53]. The fatigue behavior of cellular structures subject to dynamic loading can be broken into three stages: in Stage 1 strain increases rapidly; in Stage 2 cumulative strain remains approximately constant for 
around 104 to 106 cycles; and, in Stage 3 cumulative strain increases exponentially, eventuating in the rapid failure of the specimen [53].

Studies have shown that material type, the relative density and cell topology of cellular structures could influence their fatigue properties [54][55]. Ahmadi, S. M. et al. [56] found that the material type was far more important than the topological design in determining the normalized fatigue strength of cellular meta-biomaterials by investigating Co-Cr, Ti6Al4V, tantalum, and pure titanium materials. This is the opposite to the findings for the quasi-static mechanical properties of the same meta-biomaterials. For a given material, the cell topology of cellular structures plays a significant role for the predictions of cellular fatigue performances. Yavari, S. A. et al.[54] investigated the laser powder bed fusion porous titanium biomaterials with cube, diamond, and truncated cuboctahedron unit cells found that the type of unit cell (Fig. 1.12) and porosity both strongly influenced the fatigue lives of the cellular biomaterials. The cellular biomaterials based on the cube unit cell exhibited the longest fatigue life followed by the ones based on truncated cuboctahedron and diamond unit cells. For the effect of the relative density on the cellular fatigue properties, overall the cellular structures with higher relative density could sustain larger loads for a larger number of cycles than those with lower relative density [53]. 
a

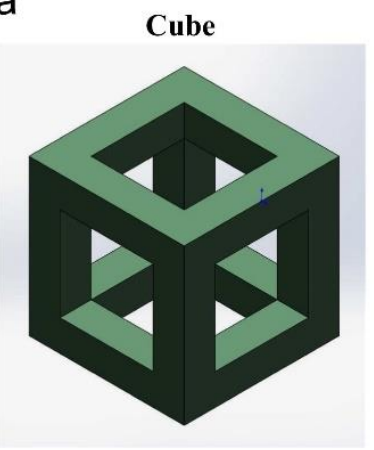

b

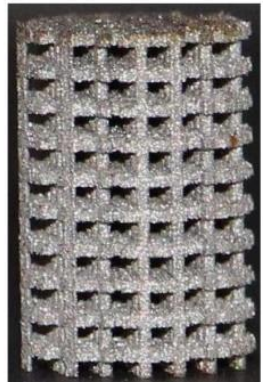

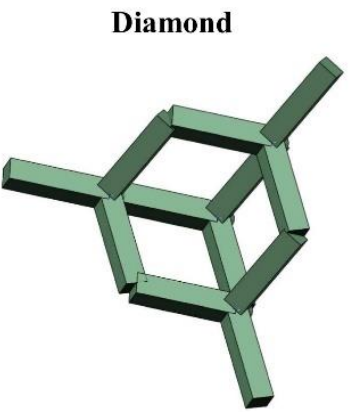

Truncated cuboctahedron
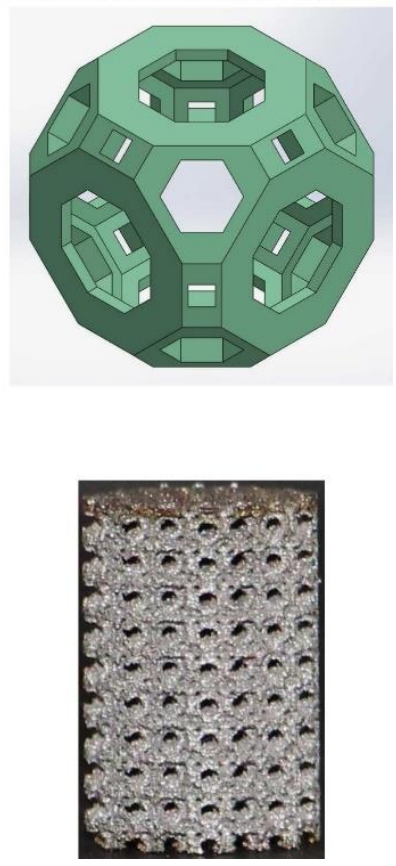

Fig. 1. 12 (a) Schematic drawings of the unit cells used for manufacturing of porous structures; (b) samples specimens from the different types of porous structures.[54]

The material type of the cellular structures also affects their fatigue properties. Titanium alloys, a popular choice for orthopedic applications, have been widely used for the fabrication of cellular structures. Studies show that the fatigue performance of Titanium cellular structure is comparable to aluminum foams [57][58][59][60]. The endurance limit of Ti6Al4V scaffolds is about $0.1-0.25$ of their yield strength. This value is less than the fatigue strength of bulk titanium alloy which is about 0.4 of yield strength [58]. This discrepancy could be contributed to the unpolished surface of struts, notch sensitivity of titanium alloy, significant porosity in struts, residual stress, and microstructure. Due to the complex geometry of the cellular structures, the mechanical treatment to improve fatigue strength (such as shot peening) is typically not practical. Instead, the heat treatment is always utilized. Leuders et al. [61] showed that hot isostatic pressing treatment could 
considerably increase the fatigue life of Ti6Al4V scaffolds. There also exists some other approaches to improve the fatigue properties of cellular structures such as stress relieving (SR), hot isostatic pressing (HIP) and chemical etching (CE) [62].

Although it is understood that both the relative density and cell topology of cellular structures affect their fatigue performance, many other factors such as manufacturing defects and cell size make the study of fatigue behavior of cellular structures remain an unexplored field [63].

\subsubsection{Failure characteristics}

Similar with the compressive and fatigue properties, the failure response of cellular structures is also highly related to their cell topology and relative density. Unlike the bulk materials, the cellular structures consist of struts or walls. The cellular structures may still have the loading capacity even after the failure of one or more struts/walls. Combining the design freedom of unit cells, the cellular structures may have various failure responses.

Amirkhani et al. [64] characterized the failure mechanism of scaffolds with different nodal connectivities (number of struts that meet in joints) and different unit cell geometries (cubic and trigonal). They found that the scaffolds with cubic unit cells and nodal connectivities of 3 and 4 showed an elastic-plastic deformation after yielding while the scaffolds with trigonal unit cells and nodal connectivities of 4 and 6 exhibited brittle behavior in the absence of pore deformation. The design rules for cellular topology selection for failure behaviors are less developed, and most of the existing knowledge is built upon specific cellular topologies. Geng et al. [65] investigated three different 3D geometries (the rhombic dodecahedron and two types of BCC cellular structures) under quasi-static tensile load by finite element method and the simulations, and they found that 
the dodecahedron cellular structures exhibit progressive fracture propagation behaviors, while for two BCC cellular structures the fracture was catastrophic. Fleck et al. [66] explored the fracture toughness of elastic-brittle 2D cellular structures by the finite element method for three periodic topologies: the regular hexagonal honeycomb, the Kagome cellular and the regular triangular honeycomb. Their study revealed that the Kagome cellular had an unusually high fracture toughness compared with the other two structures, which is due to the presence of an elastic zone of bending emanating from the crack tip into a remote stretching field. It was also observed that the hexagonal and triangular honeycombs were flaw-sensitive, while the Kagome structure was damage tolerant.

\subsection{Modeling and Manufacturing of Cellular Structures}

\subsubsection{Modeling approaches of mechanical properties of cellular structures}

Unlike the solid materials, the cellular structures are not fully dense with different topologies. Consider a tensile bar specimen of solid material under tension, its stress-strain response can be easily described which do not account for complex geometrical features. However, the cellular structures are connected by struts or wall with porosities, which lead to non-uniform local stress and strain characteristics. Besides, the cellular structures also have variable distribution of bending, stretching and shear in the connecting members, which makes it less straightforward to predict their mechanical responses. Furthermore, there exists large number of topologies for the cellular structure designs. Different cellular topologies usually exhibit different mechanical properties. For example, the BCC cellular structures exhibit a diagonal deformation pattern during compression, while the auxetic structures show a negative Poison's ratio, shown in Fig. 1.13. 

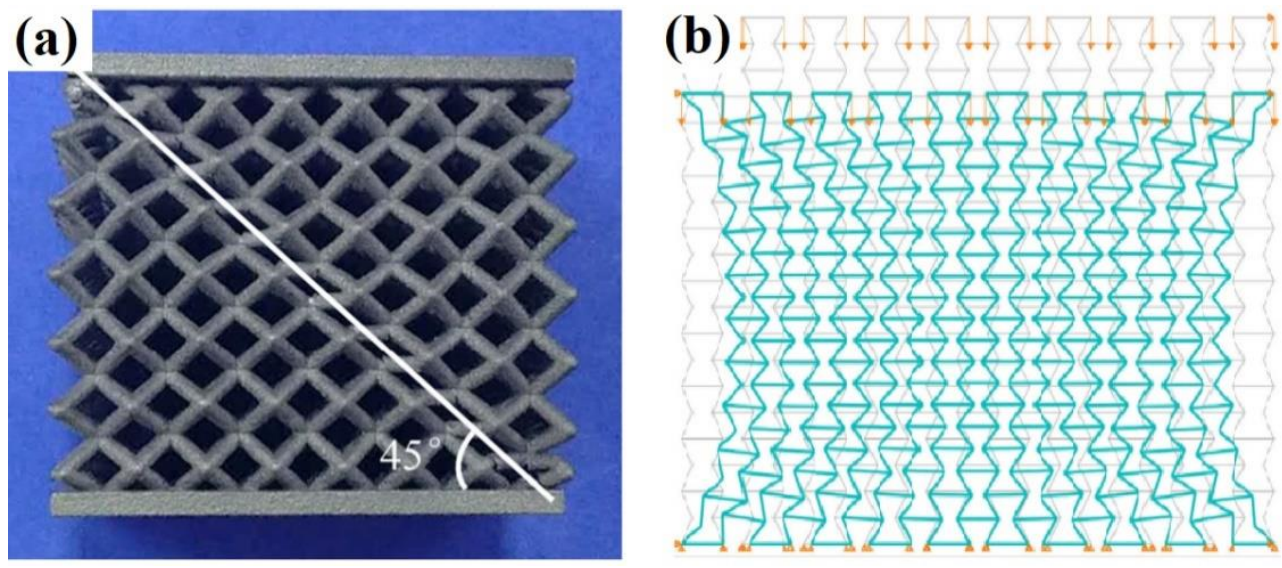

Fig. 1.13 Deformation of cellular structures under compression: (a) BCC structure [67];

(b) auxetic structure

In real-world applications, due to the limited design space, the cellular structures that are commonly designed with finite cell sizes. In addition to relative density and cell topology, the size effect, which is introduced by the boundary conditions, is another important design factor. It is common in the field of cellular structure modeling to extract an "effective" property - a property that represents a homogenized behavior without explicitly modeling the cellular detail. This is an elegant concept but introduces some practical challenges in implementation. Inherent to the assumption is that these properties, such as modulus or strength, are constant at every material point. However, in reality these properties are strongly dependent on the number of unit cells involved in the experimental characterization process. When considering the size effects, each individual unit cell and strut/wall will behave different with the other one, which makes the analytical modeling more complex. Besides, the cellular structure properties are also influenced by the boundary conditions. For example, under compression test, tensile test or fracture test, there exists physical contact between the platen and the specimen, which creates a local constraining effect at the top and bottom that is different from that on the cells closer the 
center. Although this is tied to the size effect discussed above, as when a cellular structure have large number cells in the axial direction, the contribution of the boundary effect tends to reduce, it should still be considered a separate effect for two reasons: Firstly, it raises the question of how best to design the interface for the cellular samples: whether the cellular section of the sample should be fabricated together with the grip section of the sample. Secondly, it raises the question of how best to model the interface, especially if one is seeking to match analysis results to experimentally observed behaviors.

The Gibson-Ashby [68] model is the most commonly accepted model for the prediction of the properties of cellular structures. Based on the Gibson-Ashby model, the mechanical properties of the cellular structures can be described using the structure's relative density and the raw material properties, shown in Equation (1.7)-(1.10). $E^{*}, \sigma^{*}$ and $\rho^{*}$ are the modulus, strength and density of cellular structures. $E_{S}, \sigma_{S}$ and $\rho_{S}$ are the modulus, strength and density of raw materials. $C$ is a constant which is dependent on the unit cell topology and is derived from experimental results. This model defines exponent values depending on whether the structure exhibits bending-dominated or stretchingdominated behavior, and coefficients are derived from experimental results for given unit cell topologies.

$$
\begin{array}{ll} 
& \frac{E^{*}}{E_{s}}=C\left(\frac{\rho^{*}}{\rho_{s}}\right)^{2} \\
& \frac{\sigma^{*}}{\sigma_{s}}=C\left(\frac{\rho^{*}}{\rho_{s}}\right)^{1.5} \\
& \frac{E^{*}}{E_{s}}=C\left(\frac{\rho^{*}}{\rho_{s}}\right) \\
\text { Stretching-dominated structure: } & \frac{\sigma^{*}}{\sigma_{s}}=C\left(\frac{\rho^{*}}{\rho_{s}}\right)
\end{array}
$$


Large amounts of literature have been conducted based on this model $[69][70][71][72]$. Yan et al. investigated the manufacturability and performance of advanced and lightweight stainless steel cellular structures fabricated via L-PBF

and observed increases in both modulus and strength of cellular structures with increased relative density to be consistent with expectations of the Gibson-Ashby model [73]. Zargarian et al. utilized the Gibson-Ashyb model to predict the effects of relative density, bulk material fatigue properties and cell geometry to the fatigue performance of cellular structures fabricated by L-PBF [53].

However, significant discrepancies between experimental results and theoretical predications have also been observed by several researchers [75][76][77] due to the high flexibility of the unit cell topology designs which leads to different $C$ value in Equation (1.7)-(1.10). Maconachie, T. et al. [78] summarized the extensive experimental data and compared them with the predicted data using the Gibson-Ashby model. It was found that the $C$ value in the Gibson-Ashby model for metallic open-celled bending-dominated cellular structures to be in the range of [0.1-4] and [0.1-1] for modulus and strength respectively, shown in Fig. 1.14. From Fig. 1.14, one can see some data points do not match well with the predicted values, even though most of the results were found to fall within the predicted range. Besides, all these data points are only valid for specific design parameters. It is unclear whether the Gibson-Ashby model would be applicable to all geometry design scenarios. However, the Gibson-Ashby model can still be useful in the early stages of design for providing an approximate evaluation of different cellular designs. 

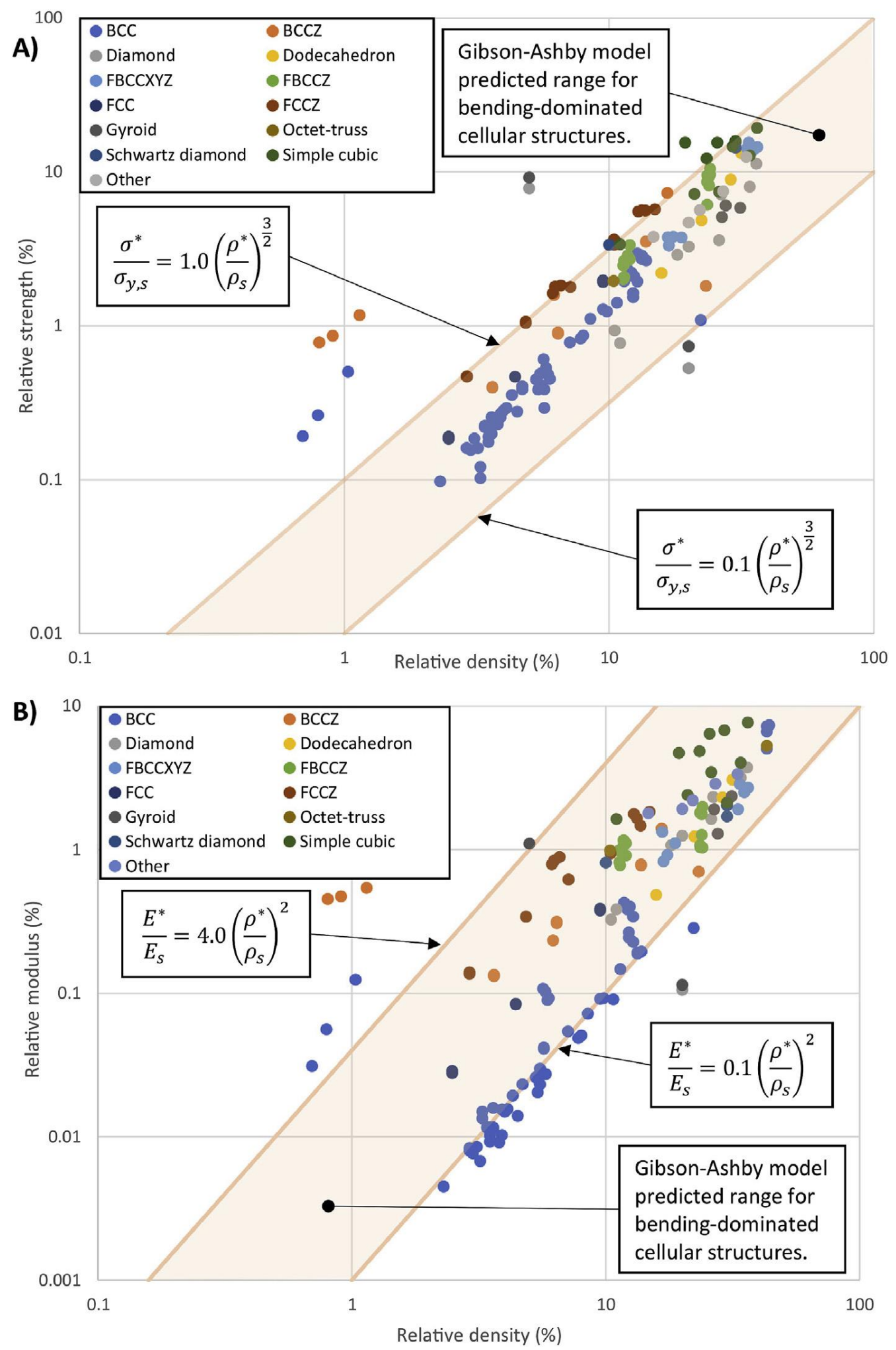

Fig. 1. 14 Comparison of reported experimental compressive strength (A) and modulus (B) data with predictions of the Gibson-Ashby model [78]. 
In many cases, although the relative density-based model could provide an initial design evaluation for the mechanical properties of cellular structures, additional modeling tools are still needed for specific topology designs. The unit cell model approach characterizes the cellular structures based on the unit cell geometry instead of the whole cellular structure. The basic concept of unit cell approach is shown in Fig. 1.15. The mechanical analysis of the periodic cellular structures is simplified to the modeling of a unit cell (Fig. 1.15(b)) with periodicity boundary conditions. Within the unit cell, the symmetry condition can be utilized to further simplify the unit cell into connected struts subjected to boundary loading conditions (Fig. 1.15(c)).

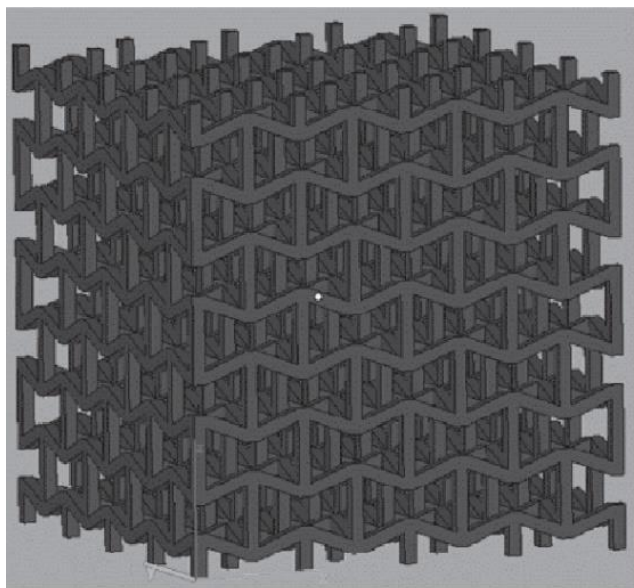

(a)

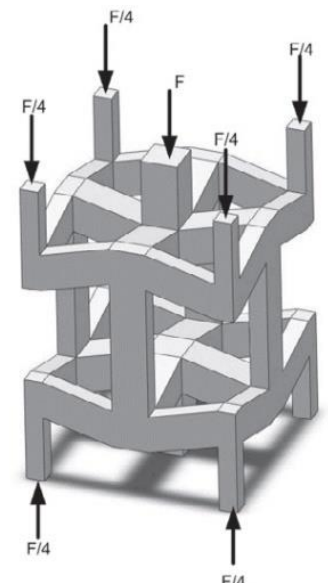

(b)

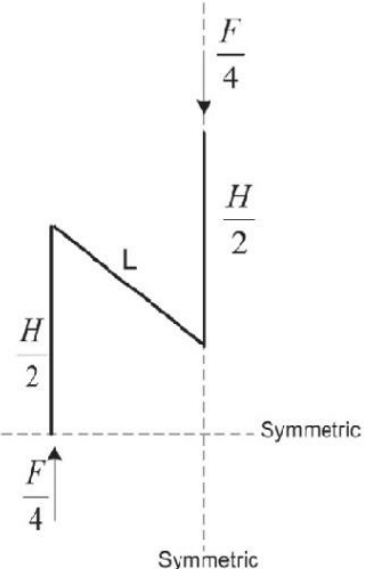

(c)

Fig. 1. 15 (a) Auxetic cellular structure; (b) Loading condition of a unit cell; (c) Loading of the simplified structure [79]

Using the unit cell model approach, the mechanical properties for cellular structures are investigated extensively by researchers [49][80][81][82]. This approach, while promising, is beset with some challenges as well: its assumption is that the cellular structure can be presented by one unit cell. For the cellular structures with infinite or larger number of unit cells, the size and boundary effect discussed above can be ignored. Then the 
mechanical behavior of cellular structure can be represented using unit cell. However, due to the limited design space, the size of the cellular structures is always relatively small, consequently, the boundary conditions could potentially exert significantly effects to the cellular deformation. This results in the differential unit cells behaviors, especially for the boundary layers and internal layers.

The development of AM technology provides more freedom for designers to create cellular structures with complex geometries and versatile functions, whose properties could be difficult to simulate with unit cell model approach. Alternatively, full-scale Finite Element Analysis (FEA) has the capability of estimating the mechanical performance of complex structures. Recently, FE modeling of cellular structures has attracted extensive attentions from researchers and has been implemented to investigate the mechanical performance of cellular structures [83][84][85]. The cellular structure is generally modeled using 2D beam elements or 3D solid elements, shown in Fig. 1.16. For the cellular structures which are connected by struts or walls, the beam element model could be utilized to model the heterogeneous cellular structures by means of varying mechanical properties, which could be caused by factors such as irregular strut thickness. An advantage of this approach is the small computational cost. However, as the 2D beam element only represent the connectivity of the structure and the equivalent mechanical properties of the cellular struts or walls, the mechanical characteristics of the joints could not be fully captured. In addition, some of the local imperfections within individual struts and walls also could not be captured. Another alternative is the 3D solid element-based approach, which tends to be more accurate when the length-to-thickness ratio (i.e. aspect ratio) of the cellular struts/walls are smaller. Compared to the beam elements, the 3D solid elements usually 
require much larger amount of mesh elements to guarantee the accuracy of the results, which corresponds to higher computational cost. On the other hand, the 3D solid element can often capture the stress-strain characteristics of the joint details as well as local imperfections of struts and walls.

(a)
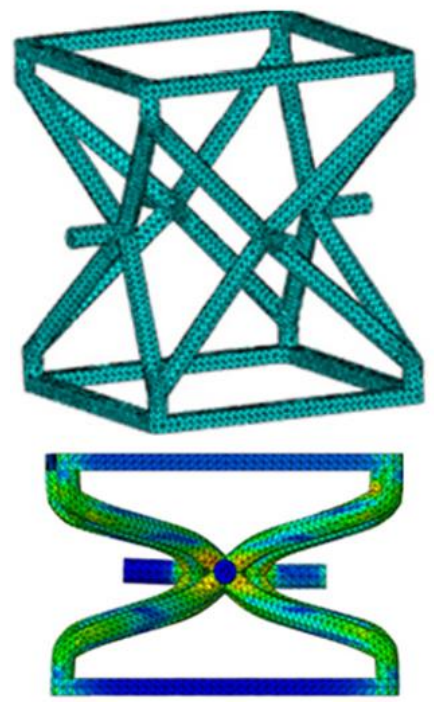

(b)
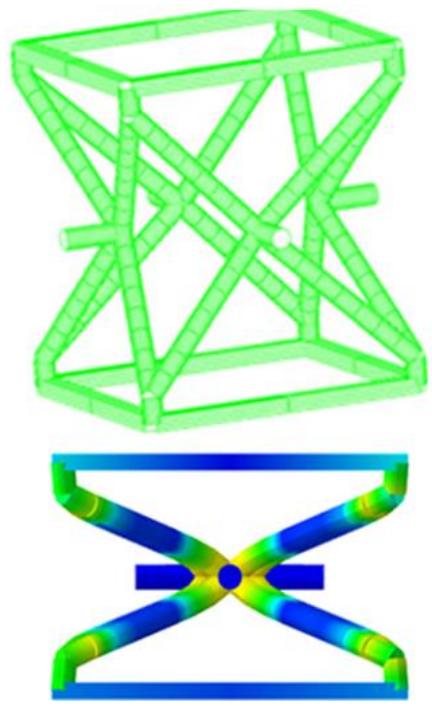

Fig. 1. 16 3D tetrahedral elements compared with beam elements: (a) 3D solid mesh using 19,830 elements and $2 \mathrm{~h} 44 \mathrm{~min}$ computational time, (b) one-dimensional beam mesh using 160 elements and $51 \mathrm{~s}$ computational time [86]

\subsubsection{Additive manufacturing of cellular structure}

\subsubsection{Additive manufacturing process}

AM process enables the fabrication of strong, lightweight structures with geometry that is unachievable by traditional manufacturing methods, including complex cellular structures. Among all the AM processes, the PBF processes such as L-PBF and electron beam PBF (EB-PBF) are commonly employed to fabricate metal cellular structures. 
A laser melting machine (shown in Fig. 1.17) distributes a layer of metal powder onto a build platform, which is melted by a laser (or multiple lasers). The build platform will then be lowered by a layer thickness and the next layer of metal powder will be coated on top. By repeating the process of coating powder and melting where needed, the parts are built up layer by layer in the powder bed at the room temperature. The technology manufactures parts in standard metals with high density (above 99\%) and good mechanical properties (comparable to traditional production technologies). A constantly widening set of standard metals is available. Parts can be further processed as any welding part.

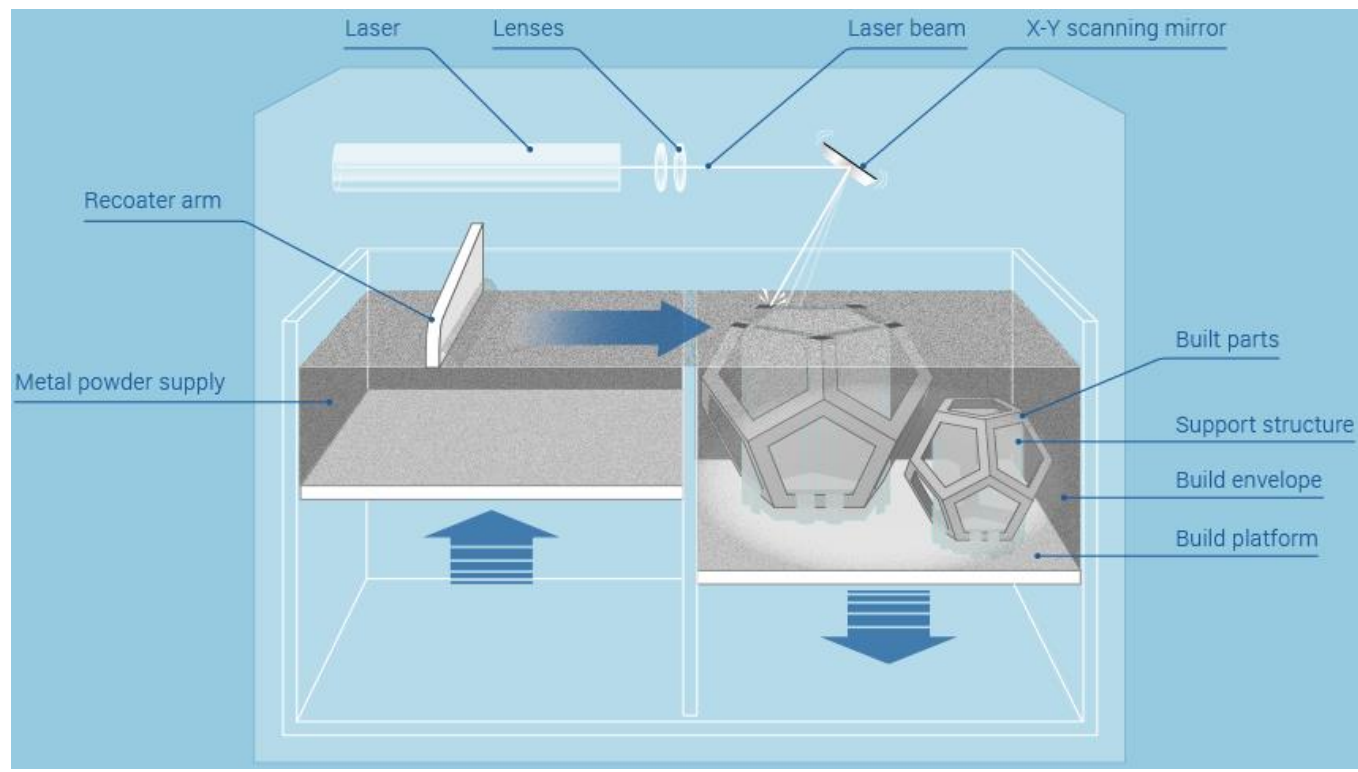

Fig. 1. 17 Layout of L-PBF system (https://www.additively.com/en/learn-about/lasermelting)

EB-PBF (Fig. 1.18) is similar to laser melting but working with an electron beam instead of a laser. The machine distributes a layer of metal powder onto a build platform, which is melted by the electron beam. This powder bed method produces fully dense metal parts directly from metal powder with characteristics of the target material. The EB-PBF machine reads data from a 3D CAD model and lays down successive layers of powdered 
material. These layers are melted together utilizing a computer-controlled electron beam. In this way it builds up the parts. The process takes place under vacuum, which makes it more capable of processing reactive materials with a high affinity for oxygen, e.g. titanium. The process is known to operate at higher temperatures (commercial system up to $1000{ }^{\circ} \mathrm{C}$ ), which can lead to differences in thermal characteristics of material in process as well as the resulting microstructure. This high temperature can also help to reduce the thermal residual stresses of the parts.

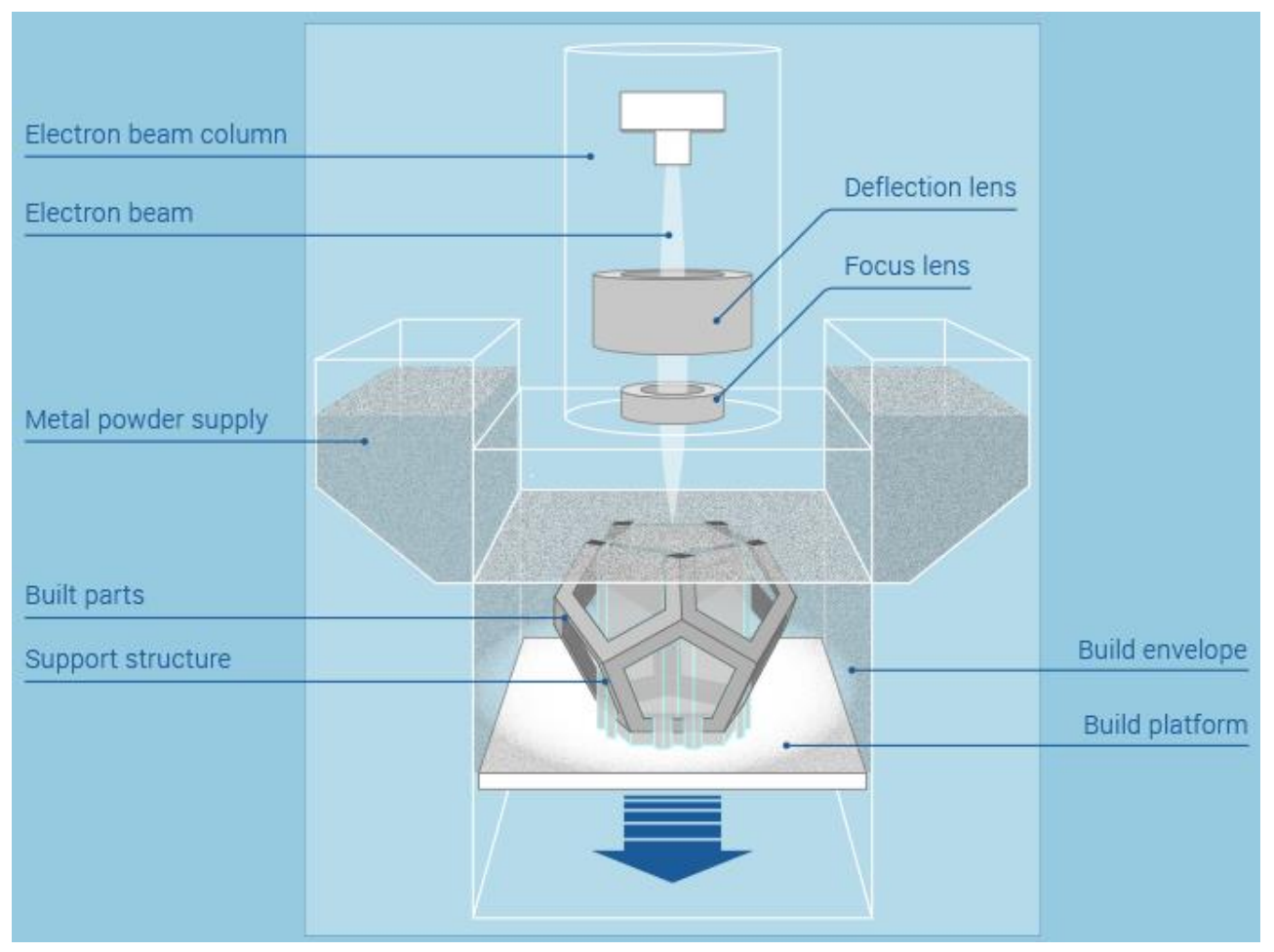

Fig. 1.18 Layout of EB-PBF system (https://www.additively.com/en/learn-about/electronbeam-melting)

\subsubsection{Defects of Additive Manufactured cellular structures}

With AM process currently recognized as arguably the most capable technologies for cellular structure fabrication, a good amount of works have been dedicated to the 
characterization of AM cellular structures such as manufacturing qualities and overall structural properties [87][88][89][90].

Due to the complex geometry of the cellular structures, they usually require support-free fabrication. However, for some cellular structures with overhanging surfaces or small inclined strut angles, the AM process may still encounter manufacturability issues. This minimum inclination angle is a function of processing parameters, material type and powder characteristics, and is often taken to be close to $45^{\circ}$ for bulk features [91]. Yan et al. [73] found that struts with an inclination angle $<30^{\circ}$ could not be manufactured by LPBF due to serious distortion. Cansizoglu et al. [92] investigated the properties of Ti6Al4V non-stochastic cellular structures with different build orientations using EB-PBF process, and suggested that the minimum orientation angle of $20^{\circ}$ is needed for the struts to ensure adequate structural integrity. Besides, the surface qualities of the cellular strut with different angles are also different. When fabricating inclined struts, the downward-facing surfaces tend to exhibit more severe surface powder attachment (e.g. partial sintering) issue in comparison to the upward-facing surface (Fig. 1.19 and Fig. 1.20). Increasing the inclination angle of downward facing surfaces reduces this effect [91].

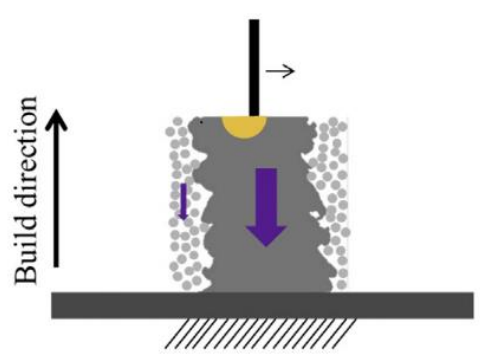

(a)

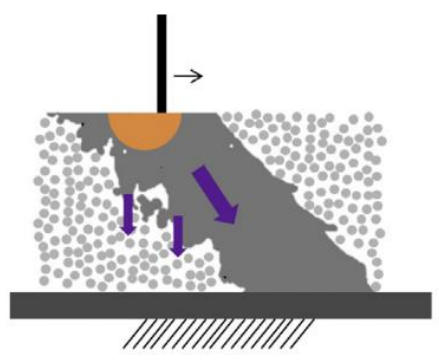

(b)

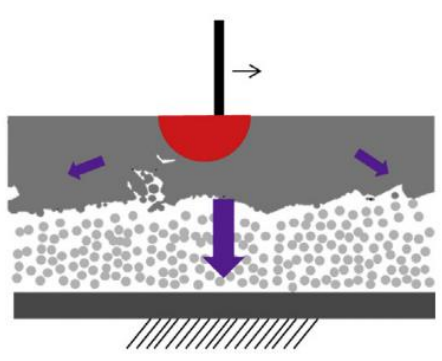

(c)

Fig. 1.19 Schematic view of the thermal behavior during EB-PBF process for different orientations: vertical (a), oblique (b) and horizontal (c). Purple arrows indicate the thermal flux density and direction [93]. 


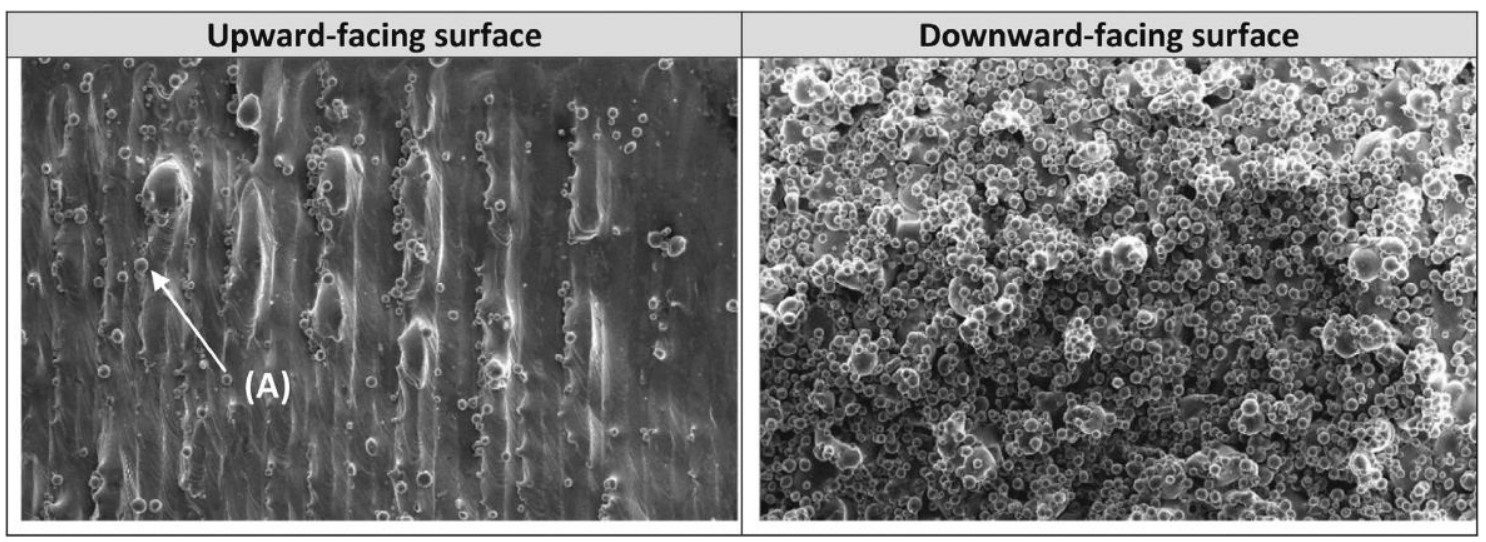

Fig. 1.20 Comparison of upward and downward-facing surface fabricated by L-PBF [78]

Since the struts within the cellular structure are often orientated at different angles, apart from their manufacturability, another important factor that becomes significant is material anisotropy. Owing to the intrinsic layerwise process nature, most AM processes produce materials with anisotropic material properties as well as anisotropic defects (e.g. surface roughness and texture) [94][95]. For examples, RubenWauthle et al. [63] explored the effect of build orientation on the microstructure and mechanical properties of laser powder bed fusion Ti6Al4V cellular structures, and observed significant decrease in mechanical strengths for samples that are built diagonally compared with the samples that are built vertically; Alsalla et al. [96] explored the effect of the build orientation on the fracture toughness of 316L stainless steel cellular structures using PBF process. The results showed that the fracture toughness of $316 \mathrm{~L}$ stainless steel cellular structure was 4.3 $\mathrm{MPa} \cdot \mathrm{m} 0.5$ in vertical (build) direction samples and 3.3 MPa $\mathrm{m} 0.5$ in horizontal directions, which was due to the reduced strength of struts oriented along horizontal direction. The staircase effect (Fig. 1.21) also partially contributes to the build orientation related defects and property variability. As shown in Fig.1.21, due to the layer-wise fabrication process, angled surfaces are fabricated with ridges of dimensions comparable to the layer thickness, 
which reduces the conformity between intended and fabricated geometries. Surface quality is reduced, necessitating post-processing procedures. Furthermore, the cellular structure has larger number of internal struts or walls which cannot be easily post-processed for defect reduction.
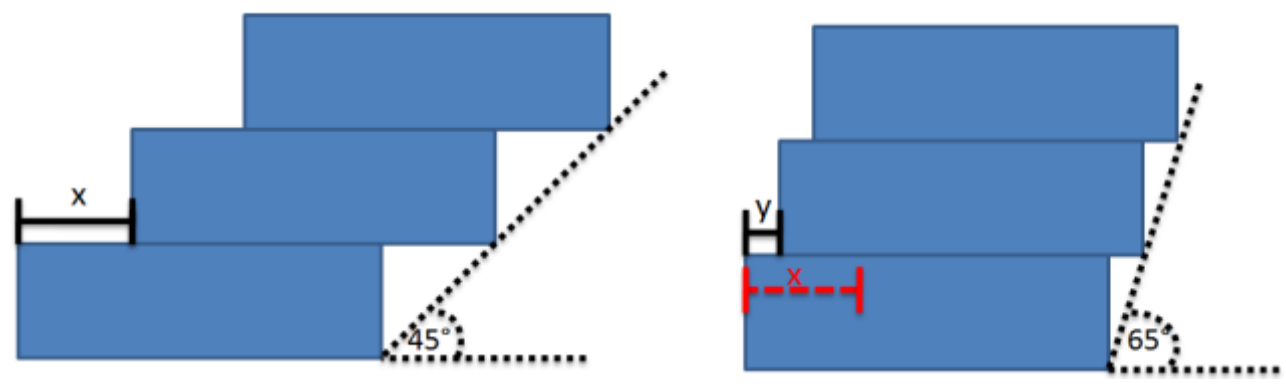

Fig. 1.21 Staircase effect depending on angle [95]

Various factors in the AM process can lead to geometrical variability of the asfabricated cellular structure from the CAD designs, such as solidification shrinkage, attachment of unmelted particles or waviness and roughness of struts. As these factors are mostly intrinsic, the mechanical properties of the as-fabricated struts almost always exhibit certain levels of variability. The variability of mechanical properties of the individual walls and struts will significantly affect the mechanical and failure response of the cellular structures. For example, with PBF process, due to the intrinsic material quality stochasticity and process instability, the fabricated materials tend to exhibit various types of surface and internal defects [97][98][99][100][101]. Table 1.1 provides a summary of the tensile test properties obtained on Ti6A14V bulk samples manufactured by directed energy deposition, L-PBF and EB-PBF. Compared with the bulk materials, the cellular structures usually have relatively smaller dimensions (sample thickness), which might lead to a higher property variability. Therefore, the property comparison (Table 1.1) of the bulk material among 
different AM processes can also provide a reference for the cellular features when using different AM processes. The longitudinal and horizontal orientations in the table indicate samples extracted in parallel and perpendicular directions to the building direction ( $\mathrm{Z}$ axis), respectively. It can be concluded from the table that there exists the mechanical property variability of the additive manufactured sample caused the defects, which cannot be avoided.

$\underline{\text { Table 1. } 1}$ Tensile properties for Ti6A14V parts built with directed energy deposition (DED), L-PBF and EB-PBF.

\begin{tabular}{|c|c|c|c|c|c|}
\hline Process & Sample orientation & $\begin{array}{l}\text { Ultimate strength } \\
\qquad \mathrm{MPa}\end{array}$ & $\begin{array}{l}\text { Yield strength } \\
\qquad \mathrm{MPa}\end{array}$ & $\begin{array}{c}\text { Elongation } \\
\%\end{array}$ & Ref. \\
\hline \multirow[t]{2}{*}{ DED } & Longitudinal & $761-821$ & $522-523$ & - & [102] \\
\hline & Horizontal & $902-923$ & $881-906$ & $\sim 6.4$ & [102] \\
\hline \multirow[t]{3}{*}{ L-PBF } & Longitudinal & $1040-1062$ & $664-802$ & $11.3-12.7$ & {$[103]$} \\
\hline & Horizontal & $1035 \pm 29$ & $910 \pm 9.9$ & $3.3 \pm 0.76$ & [104] \\
\hline & - & $960 \pm 2$ & $850 \pm 6$ & $6.8 \pm 0.5$ & [105] \\
\hline EB-PBF & Longitudinal & $851 \pm 19$ & $812 \pm 12$ & $3.6 \pm 0.9$ & [106] \\
\hline
\end{tabular}




\begin{tabular}{|c|c|c|c|}
\hline Horizontal & $833 \pm 22$ & $783 \pm 15$ & $2.7 \pm 0.4$ \\
\hline- & $790 \pm 10$ & $740 \pm 10$ & $2.2 \pm 0.3$ \\
\hline
\end{tabular}

With cellular structures, these defects could play more pronounced roles in determining the mechanical properties of the struts and walls, due to their reduced dimensions that become more comparable to the characteristic dimensions of some of the defects. Dallago, M. et at. [107] carries out in-depth investigations on the effect of the number and severity of defects on the mechanical properties using Micro X-ray computed tomography combined with the finite elements method, and it was found that both the elastic modulus and the fatigue resistance resulted strongly correlated with the number and severity of defects. Moreover, they concluded that predictions of the mechanical properties based only on the as-designed geometry were shown not to be accurate. Many other literatures also found that these process-induced defects significantly affect the mechanical response of the cellular structures [93][108][109]. This makes the pure analytical modeling less accurate when the defects information is missing. Therefore, it becomes significantly important to characterize the defects and incorporate them into cellular model.

\subsubsection{Modeling for the defects}

One commonly used approach for the analysis of the effect of defects with AM cellular structures is to incorporate equivalent properties to the modeling of struts/walls in mechanical analysis. The struts/walls are characterized first to obtain the equivalent properties. In characterizing the overall properties of the PBF cellular struts, some of the 
extrinsic defects of the struts could be accounted for by treating the struts with defects as defect-free struts with equivalent "intrinsic" material characteristics.

M. Suard et al. [93] introduced mechanically equivalent diameter of single struts for the stiffness prediction of octet-truss cellular structures produced by EB-PBF. Single struts with $1 \mathrm{~mm}$ diameter and different orientations with respect to the build direction were fabricated by EB-PBF and characterized for geometrical characteristics using X-ray tomography. Two equivalent diameters (Fig. 1.22) were defined from both inscribed cylinders from the tomographic images and numerical simulation results based on the stiffness of the reconstructed strut geometries. This method was found to yield good agreement with the experimental results on overall structural stiffness. Park et al. [108][109] proposed a similar approach that took the variability of the metal deposition of struts thickness into account in the mechanical property computation of cellular struts fabricated by PBF. Additionally, in the modeling of a re-entrant auxetic cellular structures by Yang et al. [110], a geometry-based effective strut size (Fig. 1.23) was introduced to compensate for the surface defects and joint effects of the mechanical properties of the structures. 


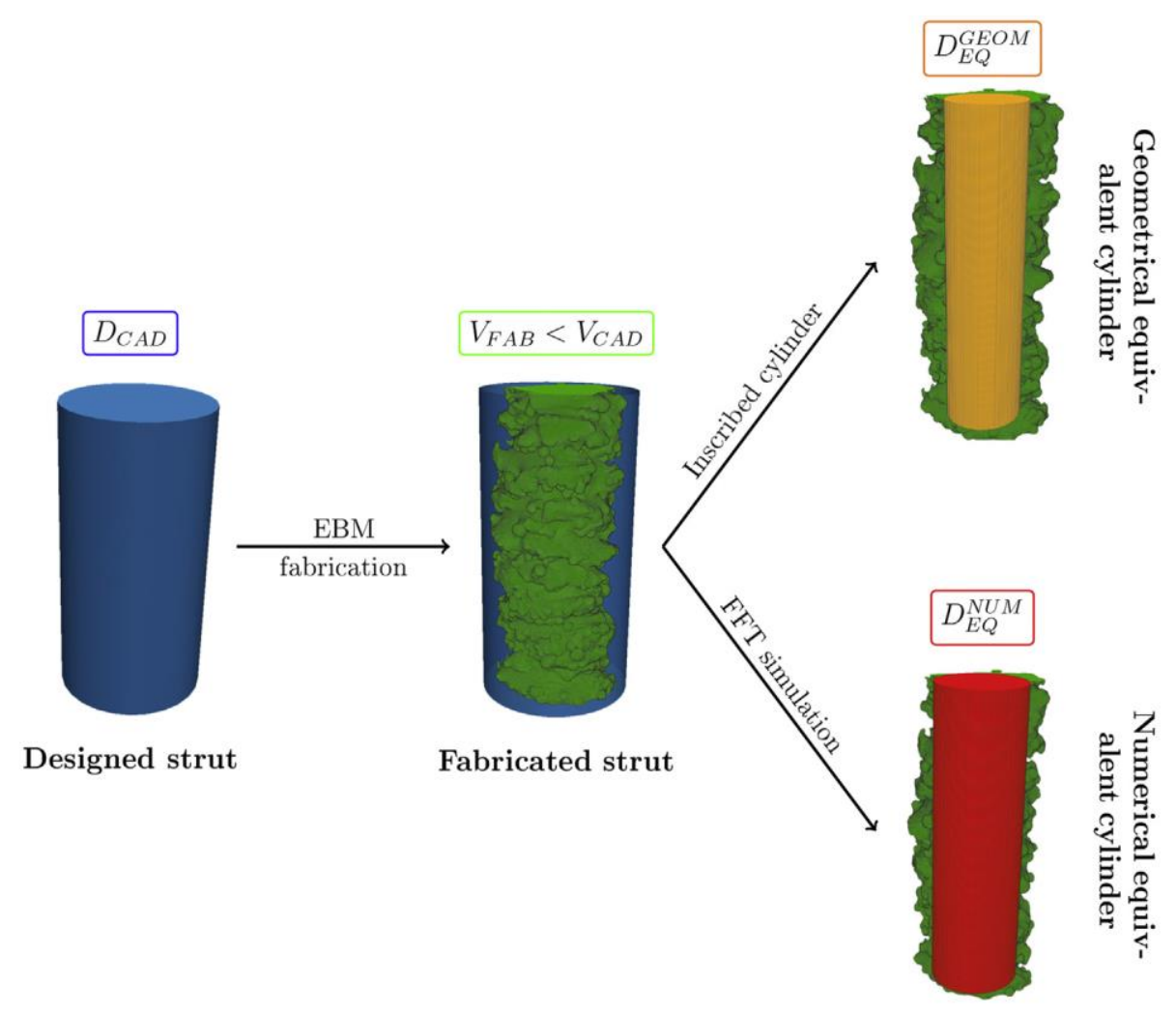

Fig. 1.22 Summary of the tools for the prediction of the effective stiffness of the struts. A designed strut (blue) is produced by the EB-PBF process. The manufactured strut (green) is smaller than the designed one. A geometrical (orange) and a numerical (red) equivalent cylinder are extracted to predict the stiffness of the produced strut. [93]

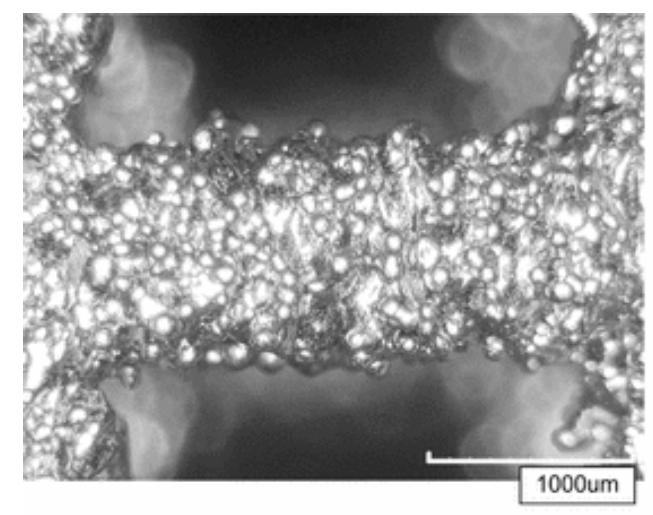

(a) Surface of a strut

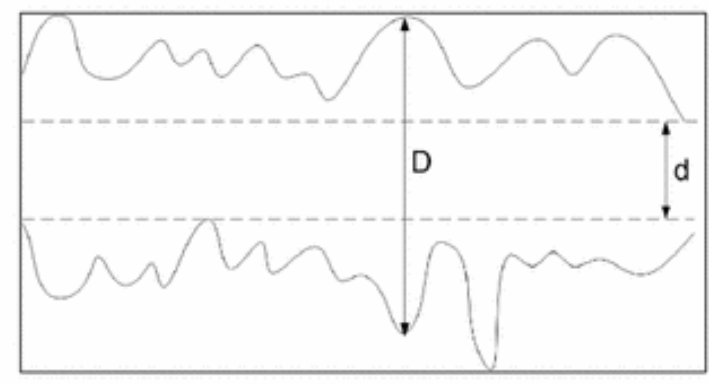

(b) Evaluation of the effective strut size

Fig. 1.23 Strut size of the parts made by EB-PBF. [110] 
Another commonly used method for the defect analysis of AM cellular structures is via direct modeling with the FEA models. The defects are identified using $\mu \mathrm{CT}$ scanning from different aspects such as the strut waviness [111], the strut diameter variability [112], strut principal axis orientations /centroid deviation [113] and internal porosity [114], and incorporated into the FEA model using various rules. Li et al. [111] analyzed the influence of the variation in cell wall thickness on the stiffness of $2 \mathrm{D}$ cellular structures and traditionally fabricated open-cell foams. The model features statistical parameters sampled from the input probability distributions of the built samples; consequently, the structural property distributions exhibit statistical distributions, shown in Fig. 1.24. It was found that both Young's modulus and shear modulus substantially deceased with the increasing variability of cell wall thickness.

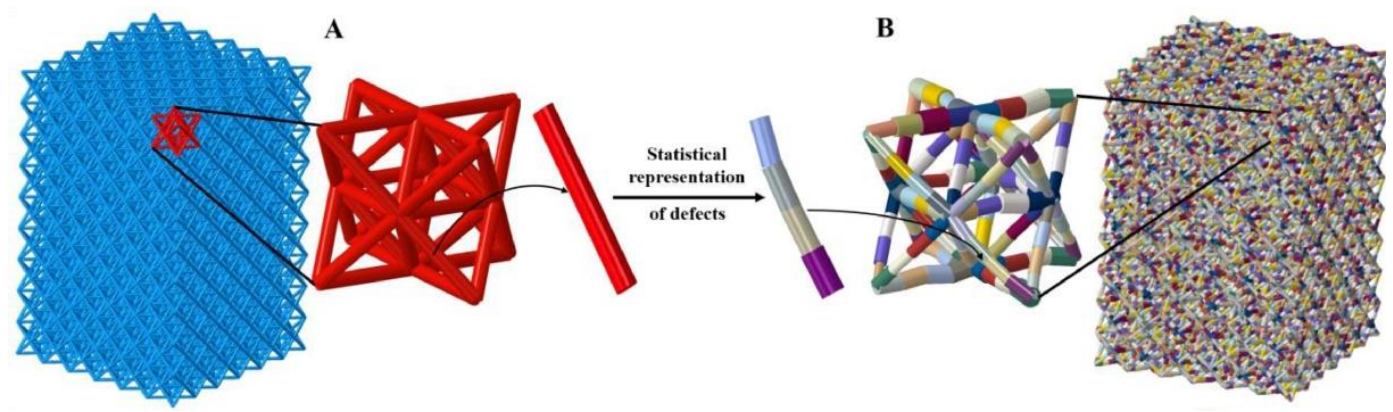

Fig. 1. 24 Finite element models: (A) numerical models with as-designed geometry, (B) numerical models with distributed geometric imperfections, showing magnified radius variations for each strut, where horizontal struts appear thicker than vertical and diagonal struts, and center axis misalignment. [111]

Overall, the equivalent diameters/properties method can provide an easy approach for the prediction of the cellular structures by building the baseline information for different strut/wall features. However, the variability among each individual strut/wall was not 
modeled, which could also lead to a not perfect accuracy. For the $\mu \mathrm{CT}$ scanning-based approach, all defects of the entire cellular structures can be modeled, which can provide a more accurate prediction for the cellular properties. However, when the cellular design changes, this $\mu \mathrm{CT}$ scanning based information will need to be recollected and recalculated, which significantly increase the computational cost. Besides, this $\mu \mathrm{CT}$ scanning based approach also tend to be inefficient in generating more generalized design knowledge when exploring large number of cellular designs.

\subsection{Current study}

In this work, the primary focus will be to establish a comprehensive model for the mechanical property prediction for the cellular structure with finite unit cell patterns. Based on this proposed model, the size-topology effect will be investigated for some commonly used 2D and 3D cellular structures.

Considering the limitations of AM process discussed in Section 1.2.2, two main factors that may potentially affect the accuracy of the proposed model, material anisotropy and material property variability, will be studied to explore the effect of the processinduced issues on the mechanical property of cellular structures.

To summarize, several objectives of this study include:

1. Modeling of relationships between design parameters and the mechanical properties of finite cellular structures;

2. the effect of unit cell size and topology on tensile failure behavior of cellular structures;

3. modeling of the effect of local material imperfection to the structural mechanical property variability of $2 \mathrm{~d}$ finite-size cellular structures. 


\section{CHAPTER II}

\section{MODELING AND CHARACTERIZATION OF CELLULAR STRUCTURES}

\subsection{Introduction}

Most of the current models for the cellular structures are concentrating on the unit cell-based approach, which can simply the boundary conditions and size effects. However, when predicting the cellular structure with finite unit cell numbers in the real world, this unit cell-based approach inevitability generates some errors. Therefore, in this section, the analytical model will be established to model the finite-size cellular structures considering both size effects and boundary conditions.

Besides, for the analytical model for the failure of cellular structure, one of the earlier modeling works by Ashby investigated the cellular structure with large number of unit cells subjected to remote stress [41]. Employing the classic linear elastic fracture mechanics modeling method, it was assumed that the critical strut directly ahead of the macroscopic crack tip would fail when its stress level reached the ultimate material strength. Such approach was also adopted by various other works as it is both mathematically efficient and conceptually convenient [66]. However, as finite-size cellular patterns often exhibit mechanical properties that are significantly influenced by the size effects, the observations and conclusions of the cellular fracture characteristics based on infinite cellular patterns are not representative under these design scenarios. The motivation of the researchers to use infinite cellular patterns in conjunction with periodic boundary conditions is to produce generic equations or conclusions regarding the behavior of cellular 
materials instead of cellular structures. However, these generic equations or conclusions cannot be directly used for the finite cellular structures. For the fracture failure analysis, due to the negligence of the potential stress concentration effects introduced by the nonideal boundary conditions, the unit cell based modeling method could cause significant errors with the critical failure strength predictions as well as the characterization of crack propagation patterns.

So the motivation of this section is to establish an analytical model for the mechanical and failure prediction of the cellular structures with finite size.

2.2 Analytical modeling of cellular structures

\subsubsection{Modeling for 2D cellular structures}

2.2.1.1 Stiffness matrix of cellular structures

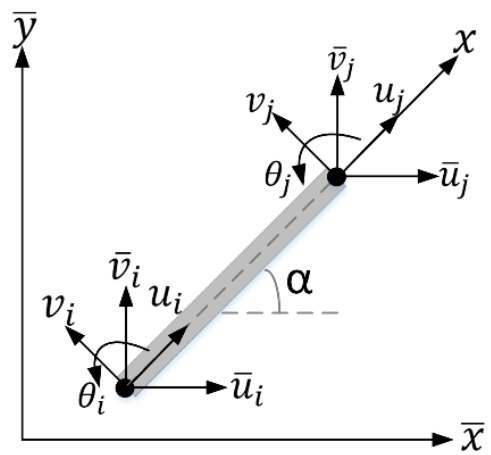

(a)

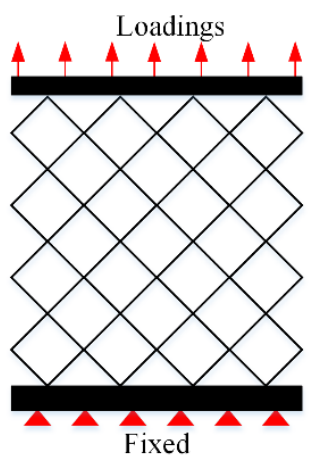

(b)

Fig. 2. 1 (a)The global coordinate system ( $\bar{x} o \bar{y})$ and local coordinate system (xoy) of the 2D beam; (b) A 2D cellular structure with loading conditions

In this study, the cellular structures are modeled as interconnected struts or walls through rigid nodes. Considering the shear deformation and rotational bending effects (which are not included in a Euler-Bernoulli beam) during the deformation of cellular 
structures, a 2D Timoshenko beam problem was described here without losing generality. For each node, there are three degrees of freedom/displacement, which are axial displacement, vertical displacement and the rotational angle. The respective forces are the axial force, shear force and the bending moment. Each wall consists of two nodes, which correspond to six degrees of freedom as shown in Fig. 2.1(a). These six displacements of the two nodes of an element can be described under both the global coordinate system $(\bar{x} \circ \bar{y})$ and local coordinate system (xoy). For the local coordinate system, the $x$ axe is set to be along the axis of the beam element from node $i$ to node $j$.

Under the local coordinate system (xoy), the forces at the ends of a beam element are related to the corresponding displacements at the ends via the element stiffness matrix, i.e.

$$
\left\{\frac{F_{i}^{e}}{F_{j}^{e}}\right\}=[\mathrm{K}]^{e}\left\{\frac{d_{i}^{e}}{d_{j}^{e}}\right\}
$$

where $d_{i}{ }^{e}$ and $d_{j}{ }^{e}$ are the displacement vectors at node $i$ and $j$ respectively, and $F_{i}{ }^{e}$ and $F_{j}{ }^{e}$ are force vectors at node $i$ and $j$ respectively for the element $i j$. The element stiffness matrix $[\mathrm{K}]^{e}$ for a six degrees of freedom wall is shown in Equation (2.1). E, A, $l$ and $I_{z}$ are Young's modulus, area of the cross section, length of the wall and the second moment of inertia, respectively. $[\mathrm{K}]^{e}$ is only dependent on the structure and material and does not rely on the applied forces. 


$$
\left[K^{e}\right]=\left[\begin{array}{cccccc}
\frac{E A}{L} & 0 & 0 & -\frac{E A}{L} & 0 & 0 \\
0 & \frac{12 E I_{z}}{L^{3}\left(1+\varphi_{y}\right)} & \frac{6 E I_{z}}{L^{2}\left(1+\varphi_{y}\right)} & 0 & -\frac{12 E I_{z}}{L^{3}\left(1+\varphi_{y}\right)} & \frac{6 E I_{z}}{L^{2}\left(1+\varphi_{y}\right)} \\
0 & \frac{6 E I_{z}}{L^{2}\left(1+\varphi_{y}\right)} & \frac{\left(4+\varphi_{y}\right) E I_{z}}{L\left(1+\varphi_{y}\right)} & 0 & -\frac{6 E I_{z}}{L^{2}\left(1+\varphi_{y}\right)} & \frac{\left(2-\varphi_{y}\right) E I_{z}}{L^{2}\left(1+\varphi_{y}\right)} \\
-\frac{E A}{L} & 0 & 0 & \frac{E A}{L} & 0 & 0 \\
0 & -\frac{12 E I_{z}}{L^{3}\left(1+\varphi_{y}\right)} & -\frac{6 E I_{z}}{L^{2}\left(1+\varphi_{y}\right)} & 0 & \frac{12 E I_{z}}{L^{3}\left(1+\varphi_{y}\right)} & -\frac{6 E I_{z}}{L^{2}\left(1+\varphi_{y}\right)} \\
0 & \frac{6 E I_{z}}{L^{2}\left(1+\varphi_{y}\right)} & \frac{\left(2-\varphi_{y}\right) E I_{z}}{L\left(1+\varphi_{y}\right)} & 0 & -\frac{6 E I_{z}}{L^{2}\left(1+\varphi_{y}\right)} & \frac{\left(4+\varphi_{y}\right) E I_{z}}{L\left(1+\varphi_{y}\right)}
\end{array}\right]
$$

In Equation (2.2), $\varphi_{y}=12 E I_{z} /\left(k_{s} A G L^{2}\right), k_{s}$ is the geometrical factor. In the case of a rectangular cross section, $k_{s}=5 / 6$.

Since $[\mathrm{K}]^{e}$ is based on the local coordinate system (xoy) as shown in Fig. 2.1(a), and for cellular structures individual walls are likely orientated differently, additional transformation is needed to convert the stiffness matrix into the more consistent global coordinate system $(\bar{x} o \bar{y})$. Therefore, the transformation matrix $[T]^{e}$ shown in Equation (2.3) was introduced to convert the different local coordinate systems to the global coordinate system.

$$
[T]^{e}=\left[\begin{array}{cccccc}
\cos \alpha & \sin \alpha & 0 & & & \\
-\sin \alpha & \cos \alpha & 0 & & 0 & \\
0 & 0 & 1 & \cos \alpha & \sin \alpha & 0 \\
& & & -\sin \alpha & \cos \alpha & 0 \\
& 0 & & 0 & 0 & 1
\end{array}\right]
$$

Apply such transformation to the applied forces and the displacements, and the stiffness matrix under global coordinate system, $[\bar{K}]$ becomes

$$
[\bar{K}]=[T]^{e T} \cdot[K]^{e} \cdot[T]^{e}
$$

Following the same procedure, one can readily assemble all the element stiffness matrices $[\overline{\mathrm{K}}]$ to obtain the stiffness matrix for the entire structure. 


\subsubsection{Boundary conditions and principal stress}

It is noted that with the established 2D cellular structure models the loadings are usually applied on the boundaries, and there does not exist external forces at the internal nodes. In this paper, the displacement "loading" was applied to model the cellular structure, as shown in Fig. 2.1(b). This means that on the boundaries the nodal displacements are known values, and the applied nodal forces (i.e. external forces on nodes) are always zero at the internal nodes. Therefore, for the $2 \mathrm{D}$ cellular structures, the displacements $[d]$ and forces $[F]$ can be grouped into the known part and unknown part for further calculation. Consequently, the stiffness matrix equation can be rewritten as

$$
\left[\begin{array}{ll}
A_{11} & A_{12} \\
A_{21} & A_{22}
\end{array}\right]\left[\begin{array}{c}
d_{\text {unknown }} \\
d_{\text {known }}
\end{array}\right]=\left[\begin{array}{c}
0 \\
F_{\text {unknown }}
\end{array}\right]
$$

where $d_{\text {unknown }}$ is the vector of unknown displacements, $d_{\text {known }}$ is the vector of known displacements, and $F_{\text {unknown }}$ is a vector of unknown forces. From Equation (2.5), the unknown displacements can be solved as

$$
d_{\text {unknown }}=-A_{11}{ }^{-1} A_{12} d_{\text {known }} .
$$

Therefore, with the knowledge of $d_{\text {known }}$, all the displacement components can be determined for the calculation of the internal forces for each wall. Finally, the stress distribution of every wall can be established from the results of the nodal displacements.

In the analysis of the maximum strength of the cellular structures, the maximum principal stress was used as the criteria for the cellular wall failure. The initial failure would occur at the wall with the maximum principal stress. This was determined by stress analysis (Fig. 2.2) with each individual beam based on the force and stress calculation results from Equation (2.1). The principal stress of individual beams is determined by both the normal 
stresses and shear stress. The normal stress is contributed by both the bending moment and axial force, while the shear stress is contributed only by the shear force.

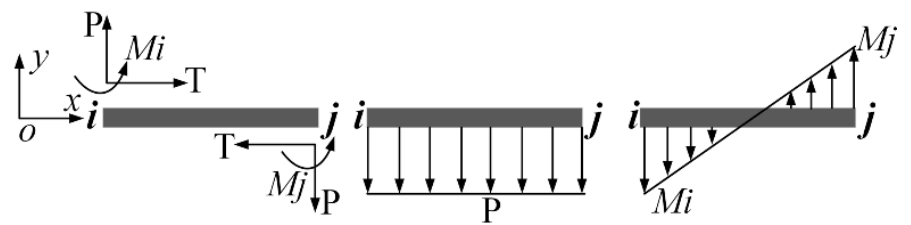

(a)

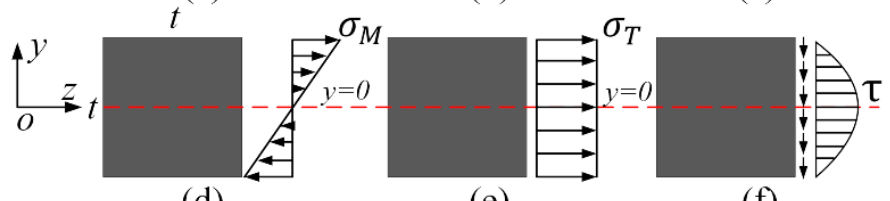

(d)

(e)

(f)

Fig. 2. 2 (a) The force equilibrium of the beam; (b) The shear force distributed along the beam; (c) the moment distributed along the beam; (d) The normal stress caused by moment; (e) The normal stress caused by axial force; (f) The shear stress caused by shear force

To briefly clarify about the calculations of the principal stress, the normal stress $\sigma$ and shear stress $\tau$ components of a location on the 2D beam of distance y from the neutral plane (Fig. 2.2) can be obtained as:

$$
\begin{gathered}
\tau=\frac{P}{2 I_{Z}}\left(\frac{t^{2}}{4}-y^{2}\right) \\
\sigma=\sigma_{T}+\sigma_{M}=\frac{M * y}{I_{z}}+\frac{T}{A}
\end{gathered}
$$

where $P$ and $T$ are the shear and normal forces, $y$ is the distance of the interested location to the neutral plane of the cross secion of the 2D beam (Fig. 2.2), $\sigma_{T}$ and $\sigma_{M}$ are the normal stress contributed by normal force and bending moments, respectively, Iz is the second moment of inertia of the beam cross section, or $I_{Z}=t^{4} / 12, \mathrm{M}$ is the bending moment, and $A$ is the are of the cross section. Finally, the maximum principal stress is obtained as: 


$$
\sigma_{1}=\frac{\sigma}{2}+\sqrt{\frac{\sigma^{2}}{4}+\tau^{2}}=\frac{1}{2}\left(\frac{M y}{I_{z}}+\frac{T}{A}\right)+\sqrt{\frac{1}{4}\left(\frac{M y}{I_{z}}+\frac{T}{A}\right)^{2}+\frac{P^{2}}{4 I_{z}^{2}}\left(\frac{t^{2}}{4}-y^{2}\right)^{2}} .
$$

Applying the criteria $\sigma_{1}=\sigma_{s}$, where $\sigma_{s}$ indicates the ultimate strength of the solid material, yields the final results of the critical force levels for each beam.

\subsubsection{Modeling for 3D cellular structures}

\subsubsection{Stiffness matrix of cellular structures}

The modeling approach for 3D cellular structures is similar with that of $2 \mathrm{D}$ cellular structures shown in Section 2.2.1. For 3D cellular structures, the struts are orientated in 3D space. The 3D beam element will be used to model the structural member, which generally subjected to transverse loading, axial loading, bending moment and torsional moment, shown in Fig. 2.3. In Fig. 2.3, the beam is of length $L$ with axial local coordinate $x$ and transverse local coordinate $y$ and $z$. The local transverse nodal displacements are given by $v_{i}$ and $w_{i}$ and the rotations by $\theta_{i y}$ and $\theta_{i z}$. The local axial nodal displacements are given by $u_{i}$ and the rotations by $\theta_{i x}$. The local nodal transverse forces are given by $F_{i y}$ and $F_{i z}$. The local nodal axial forces are given by $F_{i x}$. The local nodal bending moments are given by $M_{i y}$ and $M_{i z}$. And the local nodal torsional moments are given by $M_{i x}$. 


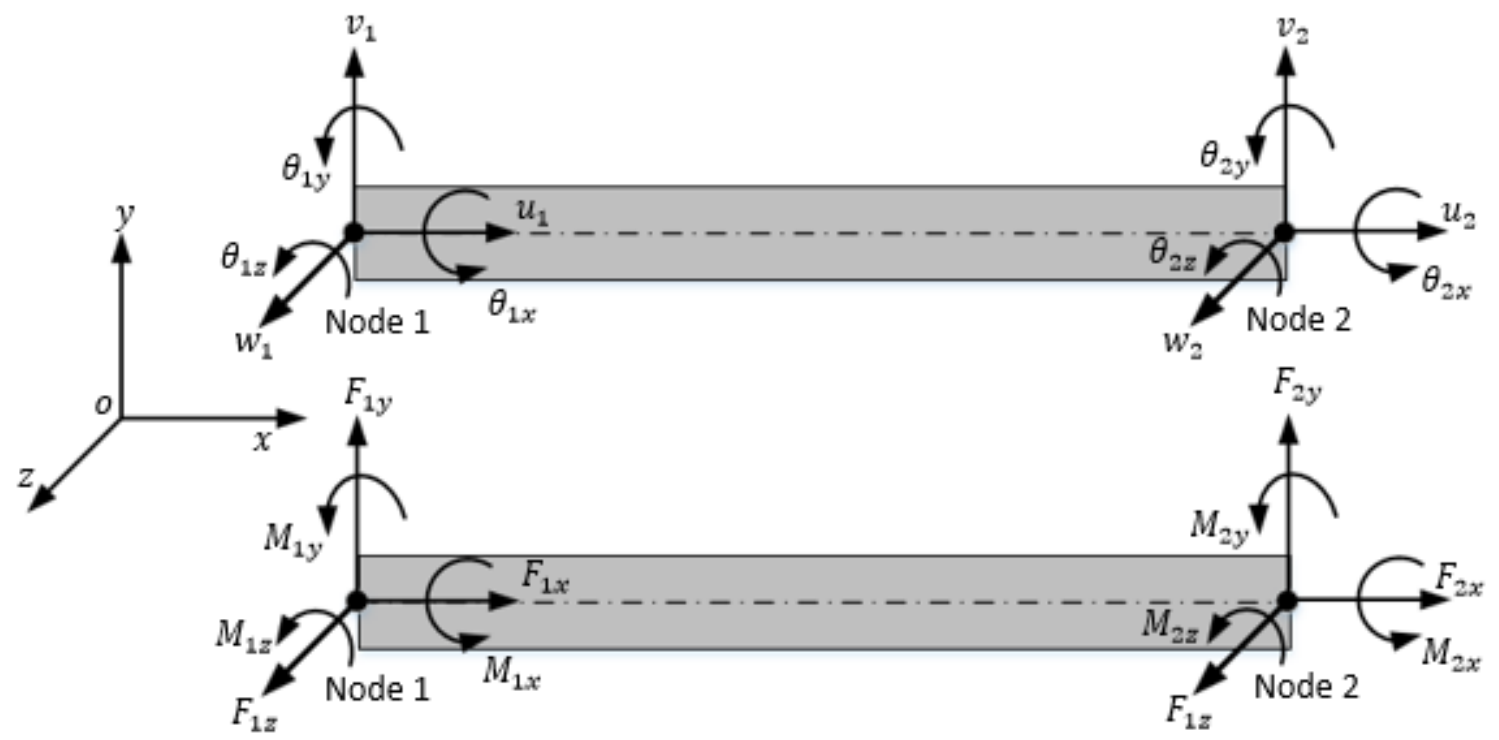

Fig. 2.3 3D Beam element with nodal displacement and nodal loadings

The same procedures in Section 2.2.1 will be used to obtain the stiffness matrix of the whole cellular structure. Due the twelve degrees of freedom of the 3D struts, the stiffness matrix will be a slightly different. Under the local coordinate system (xyz), the forces at the ends of a beam are related to the corresponding displacements at the ends by the beam stiffness matrix, i.e.,

$$
\left\{\frac{F_{i}^{e}}{F_{j}^{e}}\right\}=\left[\begin{array}{ll}
K_{i i} & K_{i j} \\
K_{j i} & K_{j j}
\end{array}\right]^{e}\left\{\frac{d_{i}^{e}}{d_{j}^{e}}\right\}
$$

where $d_{i}{ }^{e}$ and $d_{j}{ }^{e}$ are the displacement vectors at node $i$ and $j$ respectively, and $F_{i}{ }^{e}$ and $F_{j}{ }^{e}$ are force vectors at node $i$ and $j$ respectively for the element $i j$. The element stiffness matrix $[K]^{e}\left(K_{i i}, K_{i j}, K_{j i}\right.$ and $\left.K_{j j}\right)$ shown in Equation (2.10) for a single 3D beam take the forms shown in Equation (2.11)-(2.14). E, G, $A, L$ and $I$ are Young's modulus, shear modulus, area of the cross section, length of the strut and the second moment of inertia, respectively. $[K]^{e}$ is determined only by the structural topology and material and has no dependency on the applied loading conditions. 


$$
\begin{aligned}
& {\left[K_{i i}\right]=\left[\begin{array}{cccccc}
\frac{E A}{L} & 0 & 0 & 0 & 0 & 0 \\
0 & \frac{12 E I_{z}}{L^{3}\left(1+\varphi_{y}\right)} & 0 & 0 & \frac{6 E I_{z}}{L^{2}\left(1+\varphi_{y}\right)} & 0 \\
0 & 0 & \frac{12 E I_{y}}{L^{3}\left(1+\varphi_{z}\right)} & 0 & 0 & \frac{6 E I_{y}}{L^{2}\left(1+\varphi_{z}\right)} \\
0 & 0 & 0 & \frac{G J}{L} & 0 & 0 \\
0 & \frac{6 E I_{z}}{L^{2}\left(1+\varphi_{y}\right)} & 0 & 0 & \frac{\left(4+\varphi_{y}\right) E I_{z}}{L\left(1+\varphi_{y}\right)} & 0 \\
0 & 0 & \frac{6 E I_{y}}{L^{2}\left(1+\varphi_{z}\right)} & 0 & 0 & \frac{\left(4+\varphi_{z}\right) E I_{y}}{L\left(1+\varphi_{z}\right)}
\end{array}\right]} \\
& {\left[K_{i j}\right]=\left[\begin{array}{cccccc}
-\frac{E A}{L} & 0 & 0 & 0 & 0 & 0 \\
0 & -\frac{12 E I_{z}}{L^{3}\left(1+\varphi_{y}\right)} & 0 & 0 & \frac{6 E I_{z}}{L^{2}\left(1+\varphi_{y}\right)} & 0 \\
0 & 0 & -\frac{12 E I_{y}}{L^{3}\left(1+\varphi_{z}\right)} & 0 & 0 & \frac{6 E I_{y}}{L^{2}\left(1+\varphi_{z}\right)} \\
0 & 0 & 0 & -\frac{G J}{L} & 0 & 0 \\
0 & -\frac{6 E I_{z}}{L^{2}\left(1+\varphi_{y}\right)} & 0 & 0 & \frac{\left(2-\varphi_{y}\right) E I_{z}}{L^{2}\left(1+\varphi_{y}\right)} & 0 \\
0 & 0 & -\frac{6 E I_{y}}{L^{2}\left(1+\varphi_{z}\right)} & 0 & 0 & \frac{\left(2-\varphi_{z}\right) E I_{y}}{L^{2}\left(1+\varphi_{z}\right)}
\end{array}\right]} \\
& {\left[K_{j i}\right]=\left[K_{i j}\right]^{T}} \\
& {\left[K_{j j}\right]=\left[\begin{array}{cccccc}
\frac{E A}{L} & 0 & 0 & 0 & 0 & 0 \\
0 & \frac{12 E I_{z}}{L^{3}\left(1+\varphi_{y}\right)} & 0 & 0 & -\frac{6 E I_{z}}{L^{2}\left(1+\varphi_{y}\right)} & 0 \\
0 & 0 & \frac{12 E I_{y}}{L^{3}\left(1+\varphi_{z}\right)} & 0 & 0 & -\frac{6 E I_{y}}{L^{2}\left(1+\varphi_{z}\right)} \\
0 & 0 & 0 & \frac{G J}{L} & 0 & 0 \\
0 & -\frac{6 E I_{z}}{L^{2}\left(1+\varphi_{y}\right)} & 0 & 0 & \frac{\left(4+\varphi_{y}\right) E I_{z}}{L\left(1+\varphi_{y}\right)} & 0 \\
0 & 0 & -\frac{6 E I_{y}}{L^{2}\left(1+\varphi_{z}\right)} & 0 & 0 & \frac{\left(4+\varphi_{z}\right) E I_{y}}{L\left(1+\varphi_{z}\right)}
\end{array}\right]}
\end{aligned}
$$


In Equation (2.11)-(2.14), $\varphi_{\mathrm{z}}=12 \mathrm{EI}_{\mathrm{y}} /\left(\mathrm{k}_{\mathrm{s}} \mathrm{AGL}^{2}\right), \varphi_{\mathrm{y}}=12 \mathrm{EI}_{\mathrm{z}} /\left(\mathrm{k}_{\mathrm{s}} \mathrm{AGL}^{2}\right), \mathrm{J}=\mathrm{t}^{4} /$ 3 and $\mathrm{k}_{\mathrm{s}}=5 / 6$ for the square cross section.

In order to analyze the 3D cellular structure, we also need to transfer the element stiffness matrix from the local coordinate system to the global coordinate system The global coordinates of node 1 and node 2 in Fig. 2.4 are $\left(x_{1}, y_{1}, z_{1}\right)$ and $\left(x_{2}, y_{2}, z_{2}\right)$.

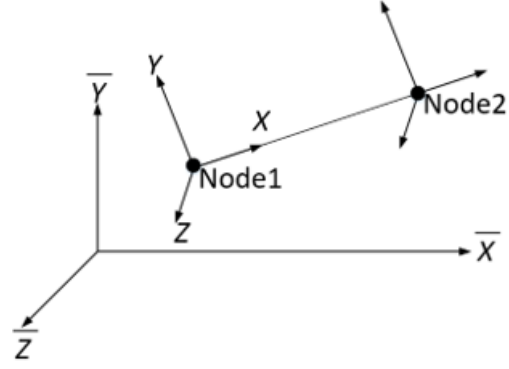

(a)

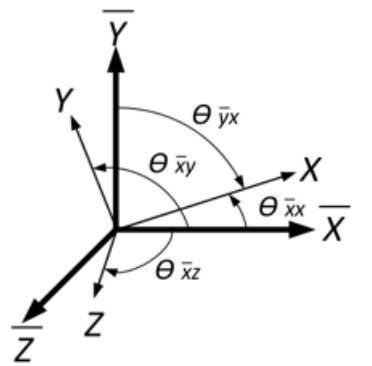

(b)

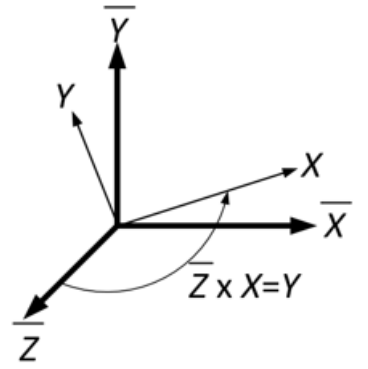

(c)

Fig. 2. 4 (a)The 3D beam under the global coordinate system; (b) Direction cosines associated with the $x$ axis; (c) Determination local y axis

The direction of the local $\mathrm{x}$ axis is the direction from node 1 to node 2 . Since the local $\mathrm{x}$ axis is determined, the local $\mathrm{x}$ can be expressed in terms of the direction cosines (shown in the Fig. 2.4(b)) as

$$
x=\cos \theta_{\bar{x} x} \boldsymbol{i}+\cos \theta_{\bar{y} x} \boldsymbol{j}+\cos \theta_{\bar{z} x} \boldsymbol{k}
$$

where

$$
\begin{aligned}
& \cos \theta_{\bar{x} x}=\frac{x_{2}-x_{1}}{L}=l \\
& \cos \theta_{\bar{y} x}=\frac{y_{2}-y_{1}}{L}=m \\
& \cos \theta_{\bar{z} x}=\frac{z_{2}-z_{1}}{L}=n
\end{aligned}
$$


The local $y$ axis is selected to be perpendicular to the local $x$ and global $\bar{z}$ axis in such a way that the cross product of global $\bar{z}$ with $x$ results in the $y$ axis, as shown in Fig. 2.4(c). Therefore,

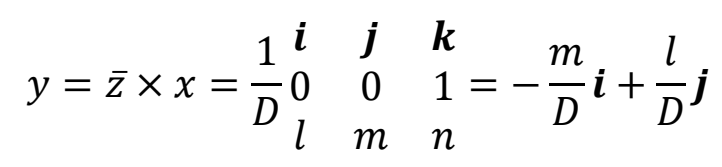

where

$$
D=\sqrt{l^{2}+m^{2}}
$$

After the local $\mathrm{x}$ and $\mathrm{y}$ are determined, the local $\mathrm{z}$ axis will be determined by the orthogonality condition as follows

$$
z=x \times y=\frac{1}{D} \begin{array}{ccc}
\boldsymbol{i} & \boldsymbol{j} & \boldsymbol{k} \\
-m & l & 0
\end{array}=-\frac{\ln }{D} \boldsymbol{i}-\frac{m n}{D} \boldsymbol{j}+\mathrm{D} \boldsymbol{k}
$$

So combing the Equation (2.15)-(2.21), we can get the transformation matrix $\left[\lambda_{3 \times 3}\right]$

$$
\left[\lambda_{3 \times 3}\right]=\left[\begin{array}{ccc}
l & m & n \\
-\frac{m}{D} & \frac{l}{D} & 0 \\
-\frac{l n}{D} & -\frac{m n}{D} & D
\end{array}\right]
$$

There are situations that we cannot use the Equation (2.22) to do the coordinate system transformation shown in Fig. 2.5. Since the local $y$ axis is determined by $y=\bar{z} \times x$, the local $\mathrm{y}$ axis will become uncertain if the local $\mathrm{x}$ axis coincides with the global $\overline{\mathrm{z}}$ axis or the local $\mathrm{x}$ axis is in the opposite direction with the global $\overline{\mathrm{z}}$ axis, as shown in Fig. 2.5(a,b) respectively. 


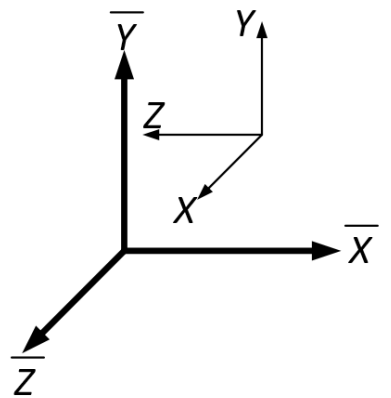

(a)

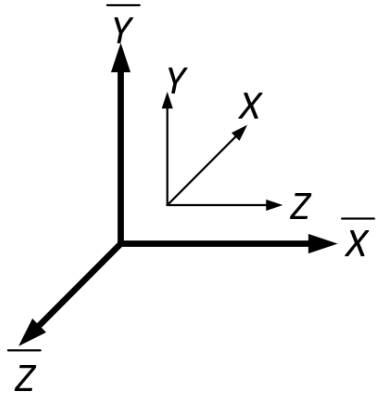

(b)

Fig. 2. 5 Special cases of the transformation matrix (a) The local $\mathrm{x}$ is in the same direction as the global $\overline{\mathrm{z}}$; (b) The local $\mathrm{x}$ is in the opposite direction as the global $\overline{\mathrm{z}}$

\subsubsection{Principal stress}

For the 3D cellular structures, the struts are oriented in space, which results in six force components for each node: the axial force $\left(F_{x}\right)$, shear force $\left(F_{y}, F_{z}\right)$, bending moment $\left(M_{y}, M_{z}\right)$ and torsional moment $\left(M_{x}\right)$ locate on two nodes of the struts. All the forces are applied to the two ends, which is shown in Fig. 2.6.

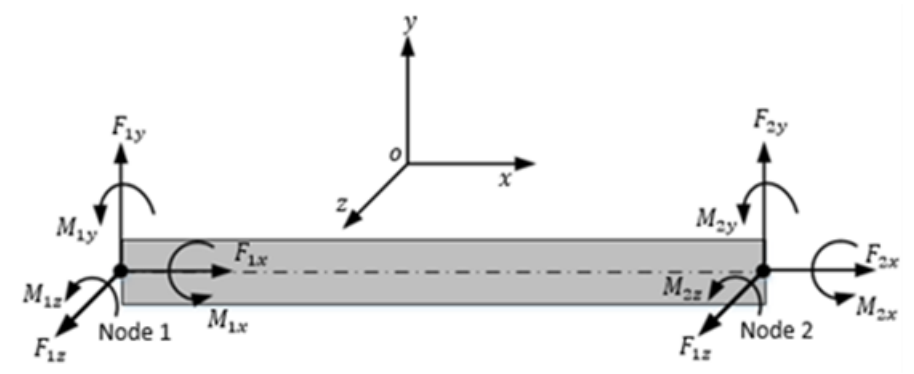

Fig. 2.6 The force components of a 3D strut

Since each strut is subject to three-dimensional stress including normal stress and shear stress. For the normal stress, there are three parts: normal stresses caused by the moment ( $M y$ and $M z)$ shown in Fig. 2.7 (a,b) and normal stresses caused by the axial force $(F x)$ shown in Fig. 2.7(c). For the shear stress, there are three parts: shear stresses caused 
by the shear forces $(F y$ and $F z)$ shown in Fig. 2.7(d,e) and shear stresses caused by the torsional moment $(M x)$ shown in Fig. 2.7(f).

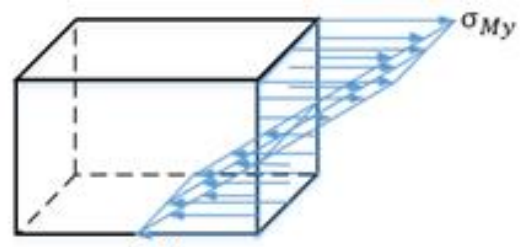

(a)
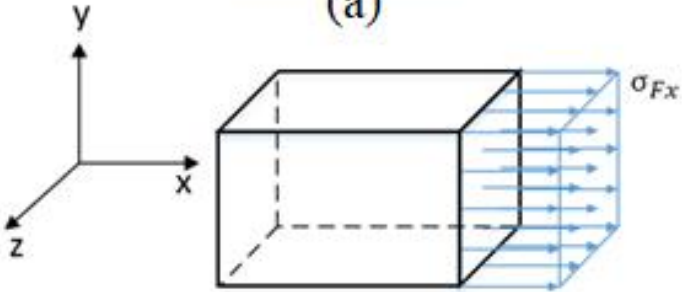

(c)

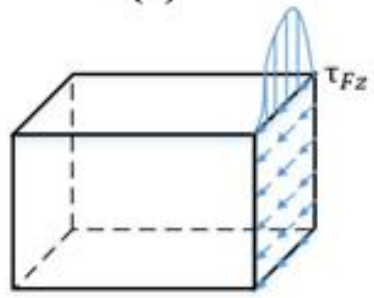

(e)

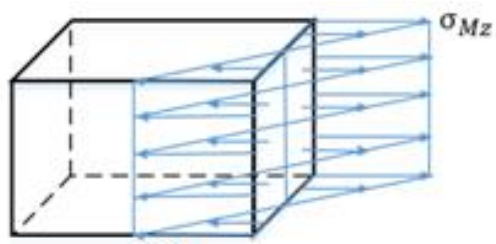

(b)

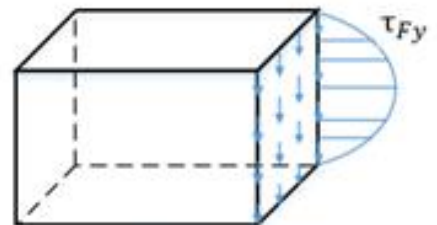

(d)

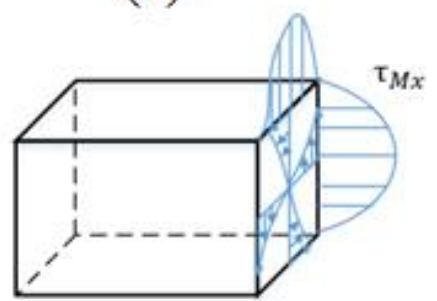

(f)

Fig. 2.7 (a) The normal stress caused by the moment $M y$; (b) The normal stress caused by the moment $M z$; (c) The normal stress caused by axial force $F x$; (d) The shear stress caused by the shear force $F y$; (e)The shear stress caused by the shear force $F z$; (f) The shear stress caused by the torsional moment $M x$;

For the normal stress caused by the moment My in Fig. 2.7(a), it is related with the moment at that plane and the distance from the neutral plane. The distribution of the normal stress caused by the moment $M y$ is shown in Fig. 2.7(a). It can be express as

$$
\sigma_{M y}=\frac{M_{y} * y}{I_{z}},
$$


where the $M y$ is the moment at each cross section, and $y$ is the distance of the point from the neutral plane. From Equation (2.23), it is obvious that the biggest $\sigma_{M y}$ is occurred at the plane which has the maximum moment.

For the normal stress caused by the moment $M z$ in Fig. 2.7(b), the same method can be used to get the normal stress

$$
\sigma_{M z}=\frac{M_{z} * z}{I_{y}},
$$

where the $M z$ is the moment at each cross section, and $z$ is the distance of the point from the neutral plane.

For the normal stress caused by the axial force $F x$ shown in Fig. 2.7(c), it can be written as

$$
\sigma_{F x}=\frac{F_{x}}{A},
$$

where the $F_{x}$ is the axial force of the beam and $\mathrm{A}$ is the area of the cross section.

For the shear stress caused by the shear force Fy shown in Fig. 2.7(d), it distributes along the cross section. The value of the stress is function of shear force $(F y)$ and the distance of the point from the neutral plane $(y)$, which can be obtained from

$$
\tau_{F y}=\frac{F_{y}}{2 I_{z}}\left(\frac{t^{2}}{4}-y^{2}\right) .
$$

For the shear stress caused by the shear force $F z$ shown in Fig. 2.7(e), it distributes along the cross section, but its direction is perpendicular to the one caused by $F y$. The value of the stress is function of shear force $(F z)$ and the distance of the point from the neutral plane $(z)$, which can be obtained from 


$$
\tau_{F z}=\frac{F_{z}}{2 I_{y}}\left(\frac{t^{2}}{4}-z^{2}\right) .
$$

For the shear stress caused by the torsional moment $M x$ shown in Fig. 2.7(f), it can be solved through the membrane analogy method. For a square cross section, the maximum shear stresses occur in the middle of the four sides shown in Fig. 2.7(f), which is

$$
\tau_{M x}=\frac{M_{x}}{0.208 t^{3}}
$$

Since each beam is subject to three-dimensional stress, the stress state for each point can be expressed with the traction vectors on a box element shown in Fig. 2.8.

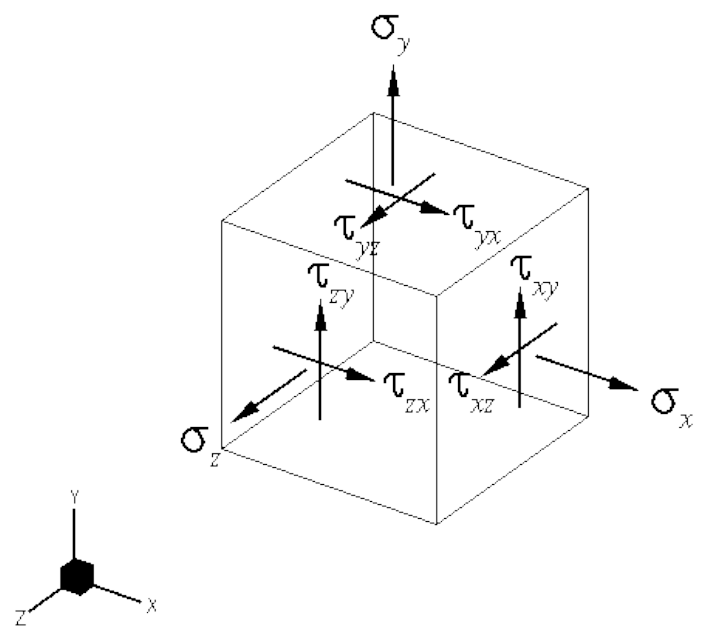

Fig. 2. 8 The traction vectors illustrated on a box element (nine stress components)

The nine stresses can be conveniently displayed in $3 \times 3$ matrix form

$$
\left[\sigma_{i j}\right]=\left[\begin{array}{lll}
\sigma_{11} & \sigma_{12} & \sigma_{13} \\
\sigma_{21} & \sigma_{22} & \sigma_{23} \\
\sigma_{31} & \sigma_{32} & \sigma_{33}
\end{array}\right]
$$

Three principal stresses can be calculated by solving the below equation

$$
\sigma^{3}-I_{1} \sigma^{2}+I_{2} \sigma-I_{3}=0
$$

where 


$$
\begin{aligned}
& I_{1}=\sigma_{11}+\sigma_{22}+\sigma_{33} \\
& I_{2}=\sigma_{11} \sigma_{22}+\sigma_{22} \sigma_{33}+\sigma_{11} \sigma_{33}-{\sigma_{12}}^{2}-\sigma_{23}{ }^{2}-\sigma_{13}{ }^{2} \\
& I_{3}=\sigma_{11} \sigma_{22} \sigma_{33}-\sigma_{11} \sigma_{23}{ }^{2}-\sigma_{22} \sigma_{31}{ }^{2}-\sigma_{33} \sigma_{12}{ }^{2}+2 \sigma_{12} \sigma_{13} \sigma_{23}
\end{aligned}
$$

For the stress state shown in Fig. 2.8, there exists the condition

$$
\begin{gathered}
\sigma_{22}=0, \sigma_{33}=0, \sigma_{32}=0 \\
\sigma_{11}=\sigma_{M y}+\sigma_{M z}+\sigma_{F x} \\
\sigma_{12}=\sigma_{21}=\tau_{F y}+\tau_{M x} \\
\sigma_{13}=\sigma_{31}=\tau_{F z}+\tau_{M x}
\end{gathered}
$$

Since the shear stress caused by torsional moment is complex function, we will evaluate same critical points of the cross section to get the maximum principal stress instead of evaluating the whole beam. From Fig. 2.8, we can see that the shear stresses and normal stresses can reach their maximum value at the four intersections or four midpoints. Then combining with the stress orientation shown in Fig. 2.8, three critical points need to be evaluated. The maximum principal stresses for these three points can be expressed as

$$
\text { Point 1: } \sigma_{1}=6 M_{y} t^{3}+6 M_{z} t^{3}+\frac{F_{x}}{t^{2}}
$$

Point 2: $\sigma_{1}=3 M_{y} t^{3}+\frac{F_{x}}{2 t^{2}}+\sqrt{\frac{\left(6 M_{y} t^{3}+\frac{F_{x}}{t^{2}}\right)^{2}}{4}+\left(\frac{3 F_{z}}{2 t^{2}}+\frac{M_{x}}{0.208 t^{3}}\right)^{2}}$

Point 3: $\sigma_{1}=3 M_{z} t^{3}+\frac{F_{x}}{2 t^{2}}+\sqrt{\frac{\left(6 M_{z} t^{3}+\frac{F_{x}}{t^{2}}\right)^{2}}{4}+\left(\frac{3 F_{y}}{2 t^{2}}+\frac{M_{x}}{0.208 t^{3}}\right)^{2}}$

The maximum one of these three values will be the critical yield strength of this beam. 
2.3 Mechanical and failure characteristics of cellular structures

\subsubsection{Mechanical properties of cellular structures}

In the previous section, both 2D and 3D cellular structures are analytically modeled through matrix stiffness method. Based on the analytical model, it is easy to establish analytical formulations for the mechanical properties. Since cellular structures could subjected to both tensile and compressive loading, their mechanical modeling approach is the same. Therefore, a uniaxial compressive loading condition for 3D cellular structure as shown in Fig. 2.9(a) is used for the demonstration for the calculation of the mechanical properties. The cellular structure is sandwiched between two rigid plates, which are "bonded" with the cellular structures and therefore represents the typical fully-constrained boundary condition of a sandwich structure with cellular core. Due to the boundary constraints, the nodal forces of nodes close to the rigid plates will likely differ from those away from the rigid plates as well as from each other, as represented by different force vectors in Fig. 2.9(b). An immediate implication of such treatment is that the classic homogenization-based modeling would be inappropriate. In addition, it was conveniently assumed that one of the plates (shown as the bottom one in Fig. 2.9(a)) was fixed, while the displacement/loading was applied on the other one.

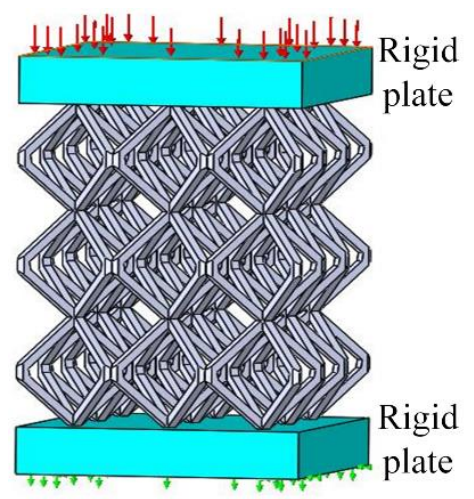

(a)Boundary condition setup

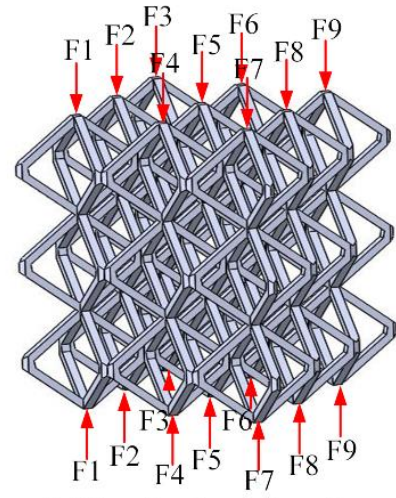

(b)Equivalent loading forces at boundaries 
Fig. 2. 9 (a) Cellular structure under uniaxial compression loading; (b) loadings on top nodes and bottom nodes

When the cellular structure reaches its critical point to fail, the compressive stress $\left(\sigma_{\text {cellular }}\right)$ can be obtained from the sum of all the normal force components on the top joints $\left(\Sigma F_{\text {top }}\right)$ and the section area of the cellular structure $\left(A_{\text {cellular }}\right)$, as shown in Equation (2.41). The strain of the structure can be obtained from the top nodel displacement $\left(d_{\text {top }}\right)$ and the length of the cellular structure $\left(L_{\text {cellular }}\right)$. Subsequently, the Young's modulus $\left(E_{\text {cellular }}\right)$ of the cellualr structure can be obtained according to Equation (2.42).

$$
\begin{gathered}
\sigma_{\text {cellular }}=\frac{\Sigma F_{\text {top }}}{A_{\text {cellular }}} \\
E_{\text {cellular }}=\frac{\sigma}{d_{\text {top }} / L_{\text {cellular }}}
\end{gathered}
$$

\subsubsection{Progressive failure modeling}

Within the scope of this paper, the initial failure in the cellular structures was set to occurs at the wall/strut with the maximum stress. Upon initial failure, the failed wall/strut is no longer contributing to the load bearing of the structures, and the stresses in the remaining cellular structure would be redistributed. Further increments in the applied loading or displacement will result in failure of other walls/struts within the structure. This progressive failure process continues until complete failure (the structure fracture into two parts) of the whole structure occurs. Such failure mode is guaranteed as in this study only tensile failure was considered.

Also within the scope of this paper, the maximum principal stress is used as the failure criteria for the determination of the progressive failure process of the cellular structures. The initial failure started at the wall/strut with the maximum principal stress. 
This was achieved by stress analysis with individual beams once the force components are determined from analytical model, shown in Section 2.2.1 and Section 2.2.2.

When the principal stress of one wall reaches the yield strength of the material, the corresponding wall/strut will fracture, which forms the initial failure. Due to the symmetry condition of the cellular structures, there will be some identical walls/struts reaching the maximum principal stress. Considering the real-world manufacturing quality variability issues from the perspective that it rarely occurs that multiple walls/struts would achieve maximum principal stress levels simultaneously, the critical wall/strut on the "upper left" part of the structures was taken as the initial failure. After the initial failure, the fractured wall/strut was removed from the cellular structures, and the stress status of the remaining structures was re-calculated. Such iterative calculations were utilized to determine the sequence of the wall/strut fracture.

To better illustrate the approach, Fig. 2.10 shows an example of the crack propagation of a finite diamond structure at different stages. The two ends of the diamond cellular structures are fully constrained, which also represents the typical boundary condition of sandwich structures or mechanical testing of cellular cubic samples. In Step 0 shown in Fig. 2.10, the diamond structure exhibits its original shape without any applied displacements. With the increase of the applied displacement, the diamond structure starts to deform. When the applied displacement reaches the first critical point, the wall 9-12 (wall that connects nodes 9 and 12) achieves maximum principal stress level that equals to the yield/fracture strength of the material and fails, which is indicated by the red dot as shown in Step 1. As the applied displacement continues to increase, there would be another wall, 13-16, that reaches the critical point and fractures as shown in Step 2. At Step 3, wall 
17-20 achieves maximum principal stress level and fractures. And right after the fracture of the wall 17-20, the stress will redistribute due to the lack of wall 17-20. At this time, the stress distribution shows that the stress level of both wall 21-24 and 1-4 exceed the yield strength of the material, which indicated that the wall 21-24 and 1-4 will fracture together with wall 17-20 and the entire structure would fail.

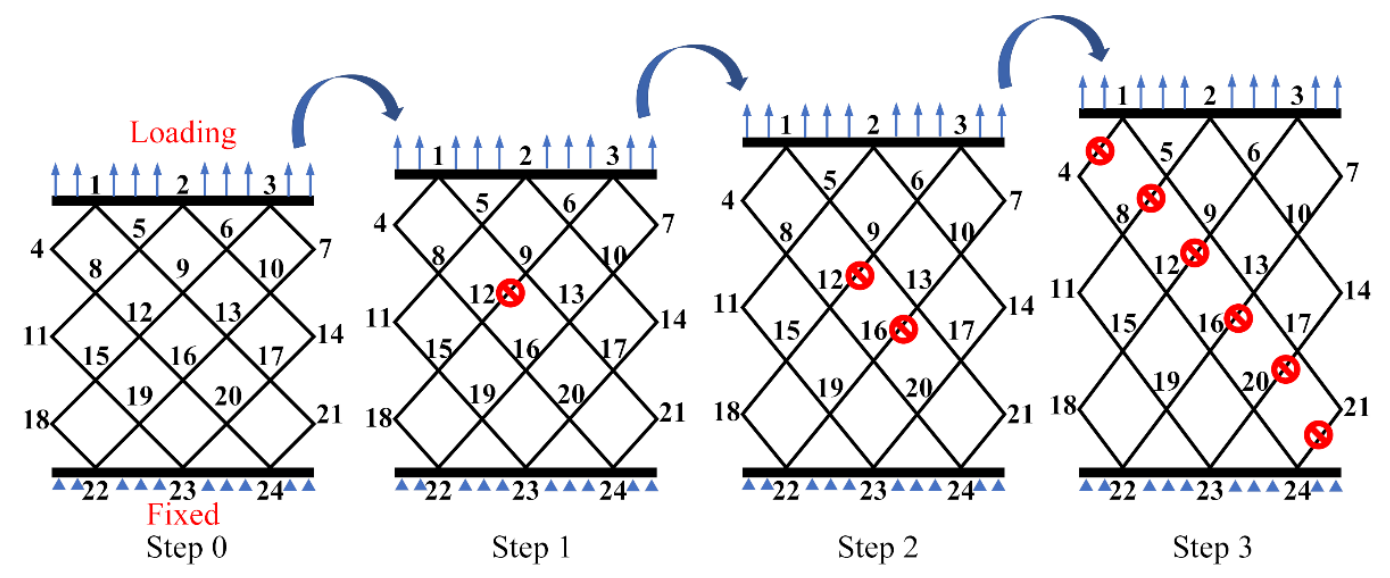

Fig. 2. 10 Demonstration of progressive failure process under tensile loading

2.4 Model verification

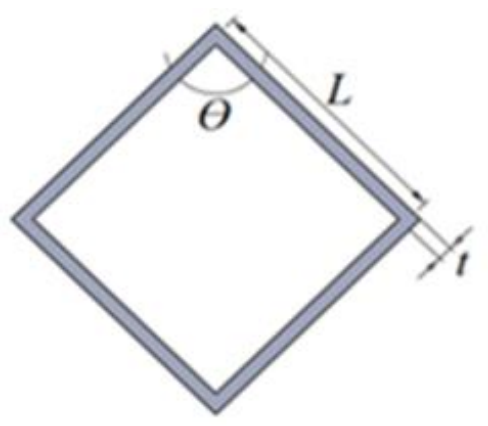

Fig. 2. 11 The detailed design parameters of diamond structure

In order to verify the above proposed processive failure model, the $2 \times 2$ and $3 \times 32 \mathrm{D}$ diamond structures were randomly chosen and designed for tensile testing. The detailed design parameters (see Fig. 2.11) are: wall length $(L)$ of $10 \mathrm{~mm}$, opening angle $(\theta)$ of $90^{\circ}$, 
wall thickness $(t)$ of $1 \mathrm{~mm}$ and wall width $(W)$ of $10 \mathrm{~mm}$. The Ti-6Al-4V powders were used to fabricate the $2 \mathrm{D}$ diamond structures with three replications for each from the EB-PBF (Arcam electron beam melting S400). The tensile tests were conducted at the Instron 5569A tensile testing machine with a constant strain rate of $0.3 \mathrm{~mm} / \mathrm{min}$. The tensile responses of the samples were recorded by a high-speed camera including the failure initiation location and progressive failure progress. For the analytical prediction based on the proposed model, the same design parameters were used with the Young's modulus of $114 \mathrm{GPa}$, the shear modulus of $43 \mathrm{GPa}$, and the yield strength of $1050 \mathrm{MPa}$. 
(a)
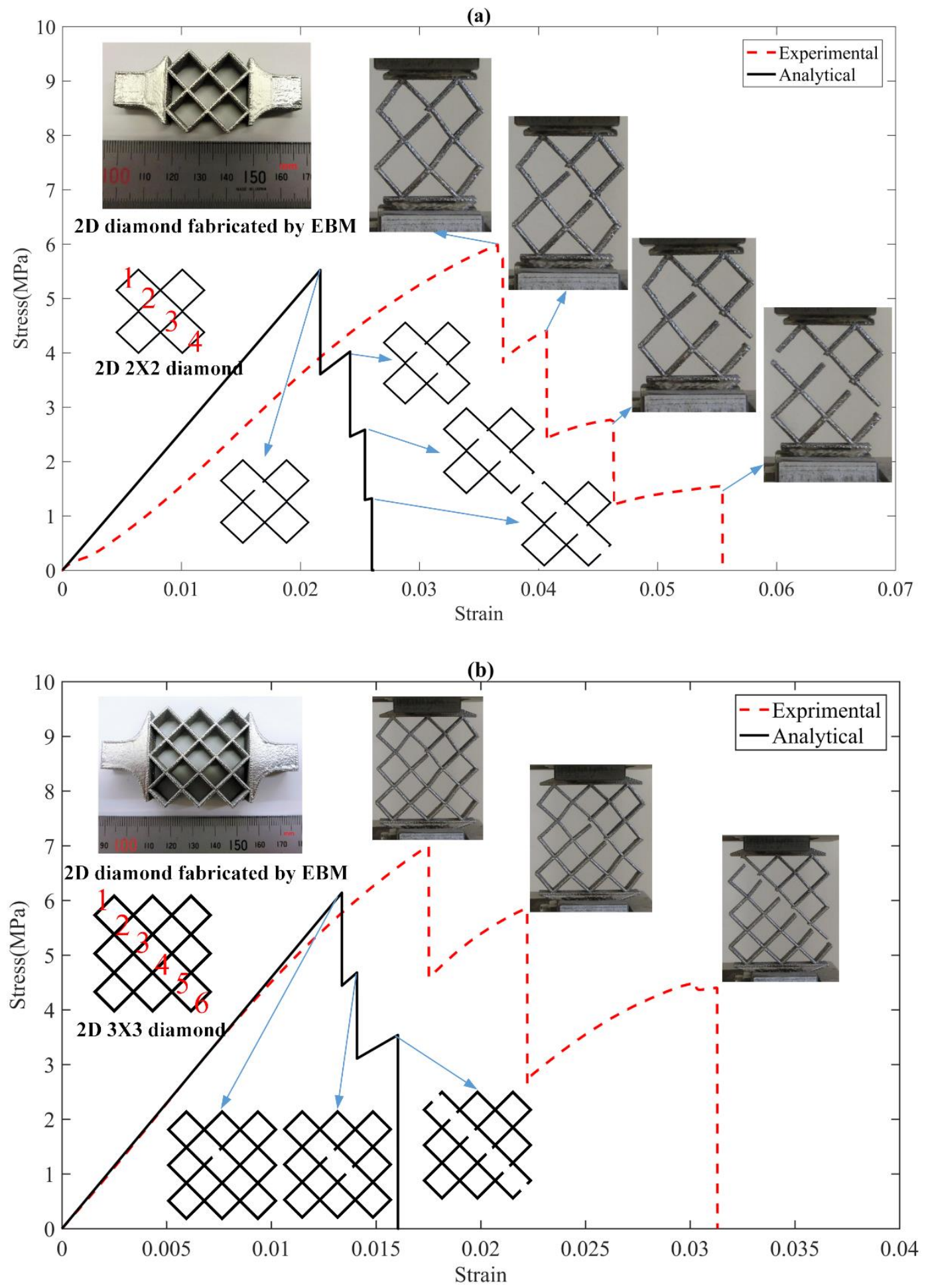

Fig. 2. 12 Comparisons between experimental and analytical results (strain-stress curve, tensile failure patterns): (a) $2 \times 2$ diamond structure; (b) $3 \times 3$ diamond structure 
Fig. 2.12 shows the tensile responses of both experiments and analytical model. In Fig. 2.12, the $\mathrm{x}$-axis and $\mathrm{y}$-axis of each curve indicate the strain and stress, respectively. Both strain-stress curves exhibit obvious saw tooth-like stress-strain patterns with multiple stress peaks. And during the tensile failure process, the critical stress levels gradually decreased. As each stress peak corresponds to the fracture of one or more walls, the recorded fractured walls from tensile test were also attached in Fig. 2.10 in order to verify the proposed progressive failure model. From Fig. 2.12a and Fig. 2.12 b (critical walls are labeled with numbers) it is clear that both experimental results and analytical results showed a very consistent tensile fracture sequence. For the $2 \times 2$ diamond structure shown in Fig. 2.12(a), the wall 2 fractured at the first peak, then the wall 3 fractured at the second peak, and then the wall 4 fractured at the third peak, and finally wall 1 fractured. For the $3 \times 3$ diamond structure in Fig. 2.12(b), the wall 3 fractured at the first peak, then the wall 4 fractured at the second peak, and finally the wall 1, wall 2, wall 5 and wall 6 fractured at the same time at the third peak. However, there existed a difference between the experiment and prediction from Fig. 2.12. The strain-stress curves of the experiment tend to be wider than that of the prediction.

Table 2. 1 Comparison of the analytical prediction and the actual experimental results

\begin{tabular}{ccccc}
\hline Design & $\begin{array}{c}\text { Analytical } \\
\text { strength }(\mathrm{MPa})\end{array}$ & $\begin{array}{c}\text { Experimental } \\
\text { strength }(\mathrm{MPa})\end{array}$ & $\begin{array}{c}\text { Analytical } \\
\text { modulus }(\mathrm{MPa})\end{array}$ & $\begin{array}{c}\text { Experimental } \\
\text { modulus (MPa) }\end{array}$ \\
\hline $2 \times 2$ diamond & 5.5 & $6.0 \pm 0.8$ & 255 & $249 \pm 1.8$ \\
$3 \times 3$ diamond & 5.3 & $6.9 \pm 0.3$ & 459 & $445 \pm 2.6$ \\
\hline
\end{tabular}


The difference between experiment and calculated stress-strain curves might be partly explained from the perspective of material model. The material model employed in the analytical model exhibits elastic-brittle characteristic. However, in reality Ti6Al4V fabricated by EB-PBF typically exhibits some plasticity after the elastic stage. Another potential source or error could come from the data acquisition source for the displacement/strain. In this work, due to the resource availability at the time of most experiments, the extensometer could not be used, and the strain was obtained from the displacement of the crosshead of the tensile testing system. Without the use of an extensometer, the rigidity difference and sample slacking between cellular section and grip section might cause some errors with strain measurements. To obtain a rough idea about the potential errors introduced by the crosshead displacement-based strain measurement, a GOM 2D digital image correlation (DIC) analysis was utilized for a limited number of samples. Since different cellular designs exhibit different rigidity levels, three randomly selected samples (one thin wall samples and two different cellular samples) were tested, which exhibit different rigidity levels. The strain values obtained from both crossheads and DIC were compared, which is shown in Fig. 2.13. It was found that the strains obtained from the DIC were around $72.3 \pm 1.5 \%$ of those obtained from the crosshead and roughly consistent across different samples regardless of their rigidity. Although the use of DIC for other samples was not available, it could be reasonably hypothesized that such error would account for $\sim 28 \%$ of the total error with strain measurements. Correspondingly, the experimental elastic modulus of different cellular structures from Table 2.1 would likely to be higher, and the stress-strain curves (Fig.2.12) would be narrower in strain range. In 
another word, most of the modulus error might be attributed to the strain calculation method adopted in this work.
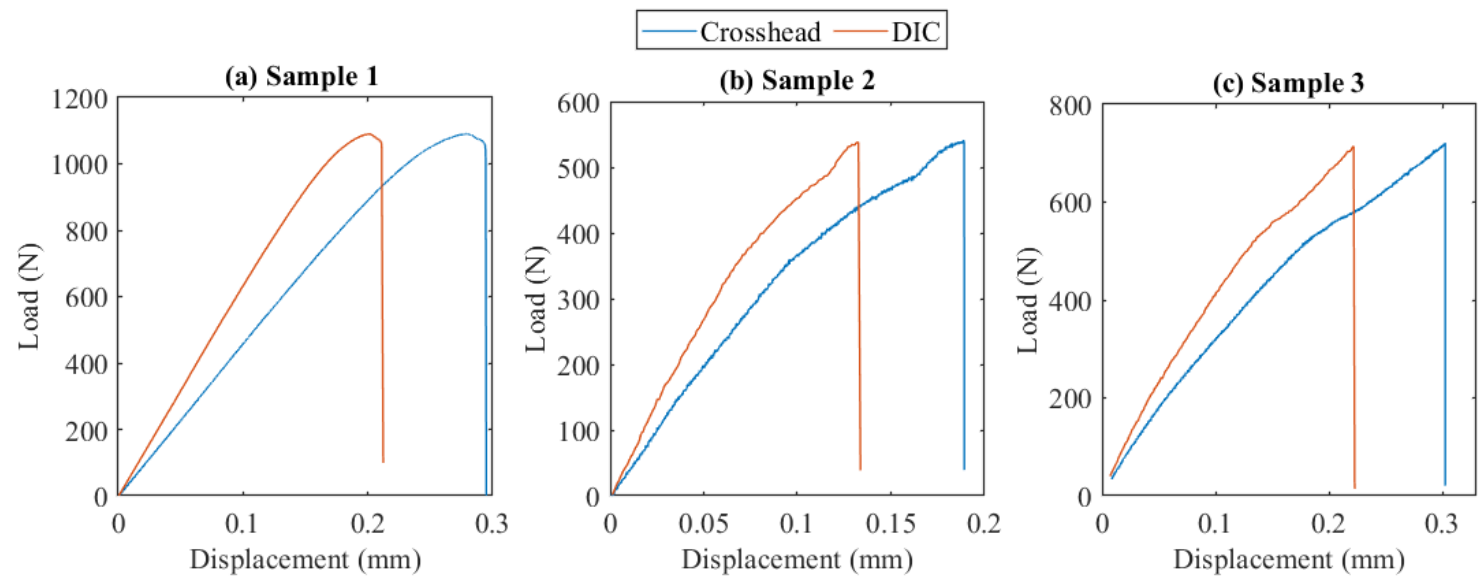

Fig. 2. 13 Displacement and load curves of three random samples

\subsection{Conclusion}

The generic equations or conclusions based on the current analytical model exhibit limitations when using for the finite cellular structures. The unit cell size effect, boundary effect and failure details are ignored in the current models. In this section, the analytical model based on stiffness matrix method was proposed to model the cellular structure with finite unit cell numbers and non-ideal boundary conditions. Then the proposed model was consequently employed for the progressive failure model of the cellular structures. In this section, the maximum principal stress criteria were proposed and then verified through experiments, which can predict the mechanical properties and failure response of cellular structures. 


\section{CHAPTER III}

\section{THE EFFECT OF UNIT CELL SIZE AND TOPOLOGY ON TENSILE FAILURE BEHAVIOR OF CELLULAR STRUCTURES}

\subsection{Introduction}

As we discussed in Section 1, it was generally recognized that the mechanical properties of cellular structures are highly dependent on both the relative density and the topologies of the unit cell designs. For the cellular structures with finite sizes that are commonly designed in real-world applications, in addition to relative density and cell topology, the size effect, which is introduced by the boundary conditions, is another important design factor. Ozdemir et al. [115][116] investigated the crushing behavior of various cellular structures including cubic, diamond, and re-entrant cube with different numbers of layers through finite element simulations and experiments. Their results showed that the compression modulus and initial yield stress are dependent on the number of layers due to the influence of weaker boundary conditions on the internal layers. Li et al. [117] discussed the influence of applied boundary conditions on the compressive characteristics of BCC cellular patterns. They found that the introduction of full constraint at both top and bottom surfaces resulted in an increase of elastic modulus by 1.5 times compared to the unconstrained conditions, indicating that the mechanical properties calculated from the isolated cellular cores cannot be directly extrapolated to predict the properties of the sandwich panel. Maskery et al. [118] experimentally investigated the compressive failure modes and energy absorption of AlSi10Mg double-gyroid cellular 
structure with various unit cell layer numbers and found that cell size plays an important role in determining the failure mechanism of metal cellular structures. Yang [119] investigated both lateral and along-the-stress size effects of multiple cellular structural designs under compressive stress using simulations. It was found that different unit cell designs exhibit significantly different size effects, and that the two types of size effects, which are induced by either free-surface or fully-constrained boundary conditions, exert different effects on the cellular structure properties.

However, size effects on the failure characteristics of cellular structures have not been explored adequately. Most of the existing literatures of cellular structure failure focus on the initial failure response of the cellular patterns employing finite element modeling (FEM) and experimentation. The fracture behaviors are generally modeled for the infinite cellular patterns with existing cracks [120][121][122][123][124][125][126][127], which is similar to the classic approach employed for the fracture analysis of solid materials. However, when using in the real engineering application, the unit cell size was always relatively small due to the limited space. Especially for the bioengineering, the failure mechanisms of the porous biomaterials were directly affected by the unit cell size and unit cell topology [48][54][128]. And most of the relevant work were based on experiments due to the lack of analytical understanding of the failure mechanisms. So the motivation of this section is to explore the failure response of the cellular structures considering the size and topology effects using the proposed model in Section 2.2. Both 2D and 3D cellular structures will be investigated in this section. 


\subsection{Size-topology effect of 2D cellular structures}

\subsubsection{Geometry design and analysis}

The progressive failure behaviors of four 2D cellular structures: auxetic, diamond, triangular1 and triangular2, as shown in Fig. 3.1 (b-e) respectively, were investigated. The choice of these four cellular structures was motivated by multiple generalized cellular structure design rules: bending/stretching dominated structures and nodal connectivity. The majority of cellular structures are bending-dominated structures due to their low nodal connectivity. However, stretch-dominated structures, typified by a fully triangulated cellular, have higher stiffness and strength compared to bending-dominated structures with the same relative density. In this paper, the auxetic structure (nodal connectivity of 3 ) is a bending-dominated structure and exhibits negative Poisson's ratio; the diamond structure (nodal connectivity of 4) is also a bending-dominated structure but exhibits regular positive Poisson's ratio; Both the triangular1 (nodal connectivity of 6) and triangular2 (nodal connectivity of 8) cellular structures are stretching-dominated structures and differs only by the degree of symmetry, with the triangular2 structure exhibiting higher geometrical symmetry. The design of these cellular structure includes five geometry parameters: the length of each wall $(L / \mathrm{mm})$ (height and length ratio $H / L$ for auxetic structure), the opening angle $\left(\theta /^{\circ}\right)$, wall thickness $(t / \mathrm{mm})$, number of unit cells in each direction $(N)$ and the wall width $(\mathrm{W} / \mathrm{mm})$, as shown in Fig. 3.1a-e. The effects of geometry parameters on the failure characteristics of four types of cellular structures under tensile stress were investigated with a constant boundary wall width of $\mathrm{W}$. Ti-6Al-4V was arbitrarily selected as the material in the analytical calculation with the Young's modulus of 114GPa, the shear modulus of $43 \mathrm{GPa}$, and the yield strength of $1050 \mathrm{MPa}$. 
(a)

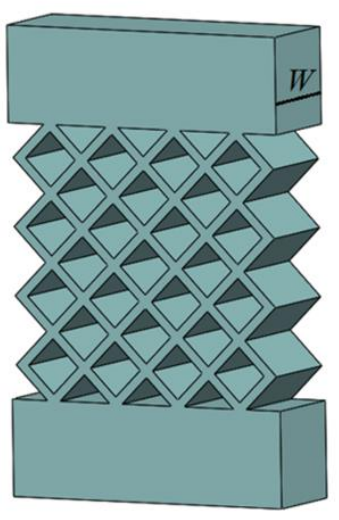

(b)

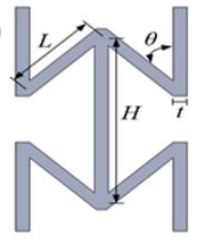

(c)

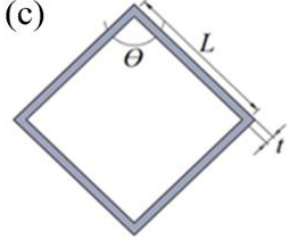

(d)

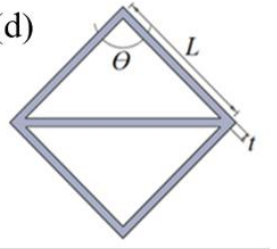

(e)

Fig. 3. 1 (a) 3D view of the 2D cellular structure; essential parameters of (b) auxetic structure; (c) diamond structure; (d) triangular1 structure; and (e) triangular2 structure

$\underline{\text { Table 3. } 1}$ Relative density (RD, \%) under design parameters for different designs

\begin{tabular}{|c|c|c|c|c|c|c|c|c|c|c|c|}
\hline \multicolumn{3}{|c|}{ 2D Auxetic } & \multicolumn{3}{|c|}{ 2D Diamond } & \multicolumn{3}{|c|}{ 2D Triangular1 } & \multicolumn{3}{|c|}{ 2D Triangular2 } \\
\hline & $\mathrm{t}$ & $\mathrm{RD}$ & & $\mathrm{t}$ & $\mathrm{RD}$ & & $\mathrm{t}$ & $\mathrm{RD}$ & & $\mathrm{t}$ & $\mathrm{RD}$ \\
\hline \multirow{5}{*}{$\begin{array}{c}\mathrm{H} / \mathrm{L}=2 \\
\mathrm{~L}=8 \\
\mathrm{O}=45 \\
\mathrm{~N}=4 \\
\mathrm{~W}=10\end{array}$} & 0.6 & 16 & \multirow{5}{*}{$\begin{array}{c}\mathrm{L}=15 \\
\Theta=90 \\
\mathrm{~N}=4 \\
\mathrm{~W}=10\end{array}$} & 0.6 & 8 & \multirow{5}{*}{$\begin{array}{c}\mathrm{L}=15 \\
\Theta=90 \\
\mathrm{~N}=4 \\
\mathrm{~W}=10\end{array}$} & 0.6 & 12 & \multirow{5}{*}{$\begin{array}{c}\mathrm{L}=15 \\
\Theta=90 \\
\mathrm{~N}=4 \\
\mathrm{~W}=10\end{array}$} & 0.6 & 17 \\
\hline & 0.8 & 22 & & 0.8 & 10 & & 0.8 & 16 & & 0.8 & 22 \\
\hline & 1.0 & 27 & & 1.0 & 13 & & 1.0 & 19 & & 1.0 & 27 \\
\hline & 1.2 & 31 & & 1.2 & 15 & & 1.2 & 22 & & 1.2 & 32 \\
\hline & 1.4 & 36 & & 1.4 & 17 & & 1.4 & 26 & & 1.4 & 36 \\
\hline & $\begin{array}{c}\mathrm{H} / \\
\mathrm{L}\end{array}$ & $\mathrm{RD}$ & & $\mathrm{L}$ & $\mathrm{RD}$ & & $\mathrm{L}$ & $\mathrm{RD}$ & & $\mathrm{L}$ & $\mathrm{RD}$ \\
\hline \multirow{5}{*}{$\begin{array}{c}t=1 \\
L=8 \\
\Theta=45 \\
N=4 \\
W=10\end{array}$} & 2 & 25 & \multirow{5}{*}{$\begin{array}{c}t=1 \\
\Theta=90 \\
N=4 \\
W=10\end{array}$} & 5 & 34 & \multirow{5}{*}{$\begin{array}{c}\mathrm{t}=1 \\
\Theta=90 \\
\mathrm{~N}=4 \\
\mathrm{~W}=10\end{array}$} & 5 & 48 & \multirow{5}{*}{$\begin{array}{c}\mathrm{t}=1 \\
\Theta=90 \\
\mathrm{~N}=4 \\
\mathrm{~W}=10\end{array}$} & 5 & 64 \\
\hline & 2.5 & 22 & & 10 & 18 & & 10 & 27 & & 10 & 38 \\
\hline & 3 & 19 & & 15 & 13 & & 15 & 19 & & 15 & 27 \\
\hline & 3.5 & 17 & & 20 & 10 & & 20 & 15 & & 20 & 21 \\
\hline & 4 & 16 & & 25 & 8 & & 25 & 12 & & 25 & 17 \\
\hline & $\theta$ & $\mathrm{RD}$ & & $\theta$ & $\mathrm{RD}$ & & $\bar{\theta}$ & $\mathrm{RD}$ & & $\theta$ & $\mathrm{RD}$ \\
\hline $\mathrm{H} / \mathrm{L}=2$ & 30 & 41 & $\mathrm{t}=1$ & 30 & 24 & $t=1$ & 30 & 28 & $t=1$ & 30 & 45 \\
\hline $\mathrm{L}=8$ & 45 & 26 & $\mathrm{~L}=15$ & 60 & 14 & $\mathrm{~L}=15$ & 60 & 20 & $\mathrm{~L}=15$ & 60 & 30 \\
\hline $\mathrm{t}=1$ & 60 & 19 & $\mathrm{~N}=4$ & 90 & 13 & $\mathrm{~N}=4$ & 90 & 19 & $\mathrm{~N}=4$ & 90 & 27 \\
\hline $\mathrm{N}=4$ & 75 & 15 & $\mathrm{~W}=10$ & 120 & 14 & $\mathrm{~W}=10$ & 120 & 23 & $\mathrm{~W}=10$ & 120 & 30 \\
\hline
\end{tabular}




\begin{tabular}{|c|c|c|c|c|c|c|c|c|c|c|c|}
\hline $\mathrm{W}=10$ & 85 & 13 & & 150 & 24 & & 150 & 39 & & 150 & 45 \\
\hline & $\mathrm{N}$ & $\mathrm{RD}$ & & $\mathrm{N}$ & $\mathrm{RD}$ & & $\mathrm{N}$ & $\mathrm{RD}$ & & $\mathrm{N}$ & $\mathrm{RD}$ \\
\hline $\mathrm{H} / \mathrm{L}=2$ & 2 & 26 & \multirow{5}{*}{$\begin{array}{c}t=1 \\
L=15 \\
\Theta=90 \\
W=10\end{array}$} & 2 & 13 & \multirow{5}{*}{$\begin{array}{c}t=1 \\
L=15 \\
\Theta=90 \\
W=10\end{array}$} & 2 & 19 & \multirow{5}{*}{$\begin{array}{c}t=1 \\
L=15 \\
\Theta=90 \\
W=10\end{array}$} & 2 & 27 \\
\hline $\mathrm{t}=1$ & 3 & 26 & & 3 & 13 & & 3 & 19 & & 3 & 27 \\
\hline $\mathrm{L}=8$ & 4 & 26 & & 4 & 13 & & 4 & 19 & & 4 & 27 \\
\hline$\Theta=45$ & 5 & 26 & & 5 & 13 & & 5 & 19 & & 5 & 27 \\
\hline $\mathrm{W}=10$ & 6 & 26 & & 6 & 13 & & 6 & 19 & & 6 & 27 \\
\hline
\end{tabular}

From Table 3.1, it can be seen that the relative density is almost proportional to the wall thickness $t$ for the four structures. The thicker the wall thickness, the more the relative density will be. Similarly, the relative density also decreases almost linearly with the increases of the wall length. On the other hand, for the $2 \mathrm{D}$ diamond structure, $2 \mathrm{D}$ triangular1 structure, and 2D triangular2 structure, the relative densities exhibit the lowest values when the opening angles are at $90^{\circ}$ and increase monotonously as the opening angles deviate more from $90^{\circ}$. For the $2 \mathrm{D}$ auxetic structure, the relative density keeps decreasing when the opening angle varies from $30^{\circ}$ to $85^{\circ}$. Furthermore, the relative density remains the same under different unit cell numbers since the geometry parameters of each unit cells are identical.

\subsubsection{Size and topology effects on mechanical properties}

Fig. 3.2 shows the stress-strain curves for the four types of cellular structures of different geometrical parameters using the analytical model described in Section 2.2.1. In Fig. 3.2, the x-axis and y-axis of each curve indicate the strain and stress respectively. At each failure step, the strain-stress curves of the wall failure exhibit the perfect elasticcatastrophic failure characteristics typical to the brittle materials with maximum stress failure mode that is adopted in this study. The two bending-dominated diamond structures, 2D auxetic and 2D diamond structures, exhibit obvious saw tooth-like stress-strain patterns 
with multiple stress peaks during the tensile failure process, with critical stress levels gradually decrease. On the other hand, both stretching-dominated structures, 2D triangular1 and 2D triangular2 structures, exhibit essentially only one stress peaks. As each stress peak corresponds to the fracture of one or more walls, multiple peaks indicate that the fracture is gradual, or that the crack propagation of the structures is more stable. Therefore, for the bending-dominated diamond structures, after one or multiple walls fail at each step, the remaining structures still exhibit some structural integrity and strength for further load application.

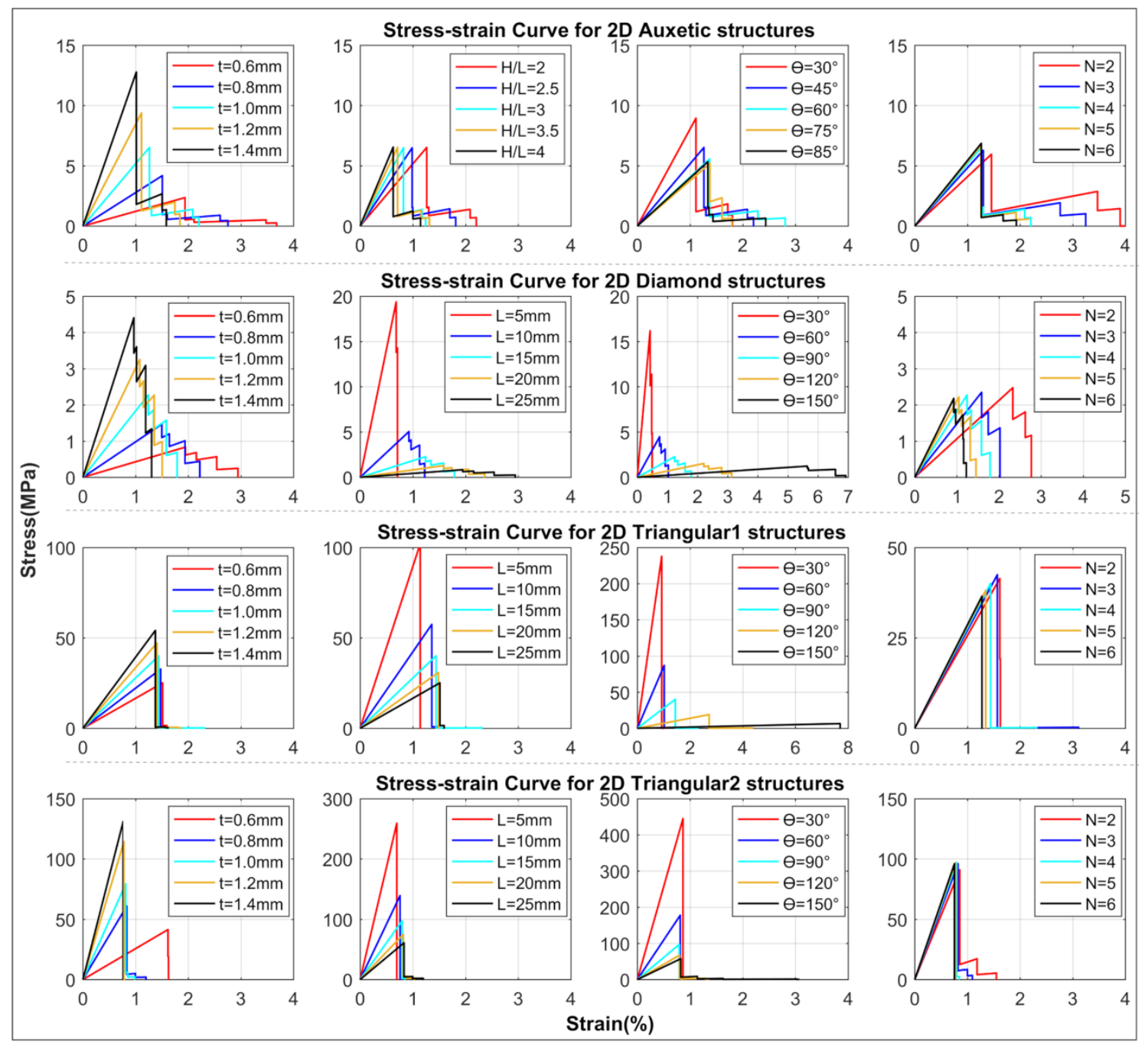

Fig. 3.2 Stress-strain curves for different cellular structures under different parameters 
From the results, it is also obvious that the failure strengths and elastic modulus of all the cellular structures exhibit significant dependency on their respective geometry design parameters. As can be seen from Fig. 3.2, both the failure strength and the elastic modulus of the cellular structures increase when the increase of wall thickness, decrease of the wall length, and the decrease of the angle $\theta$. This is intuitive as the change of these geometry parameters also correspond to the change of the relative densities of the cellular structures, which is known to strongly influence their properties. Another obvious finding from the stress-strain curves is the relationship between design parameters and tensile strength/ Young's modulus. The tensile strength of a structure is defined as the maximum stress before the first occurrence of a fracture. The Young's modulus was obtained by taking the slope of the linear portion of the first stress-strain curve period. For all four types of the cellular structures, the tensile strength increases, and the Young's modulus decreases with an increase of the wall thickness and a decrease of opening angle. With the increase of the length of the walls, the $2 \mathrm{D}$ auxetic structures exhibit increasing trends with both tensile strength and Young's modulus, while the 2D diamond, 2D triangular1 and 2D triangular2 structures exhibit decreasing trends with tensile strength and increasing trends with Young's modulus. On the other hand, only the elastic modulus of the 2D diamond exhibits significant increasing trend with increased number of unit cells, the detailed size and topology effects on tensile strength and Young's modulus will be discussed further in section. 
3.2.2.1 Size and topology effects on initial tensile strength and Young's modulus

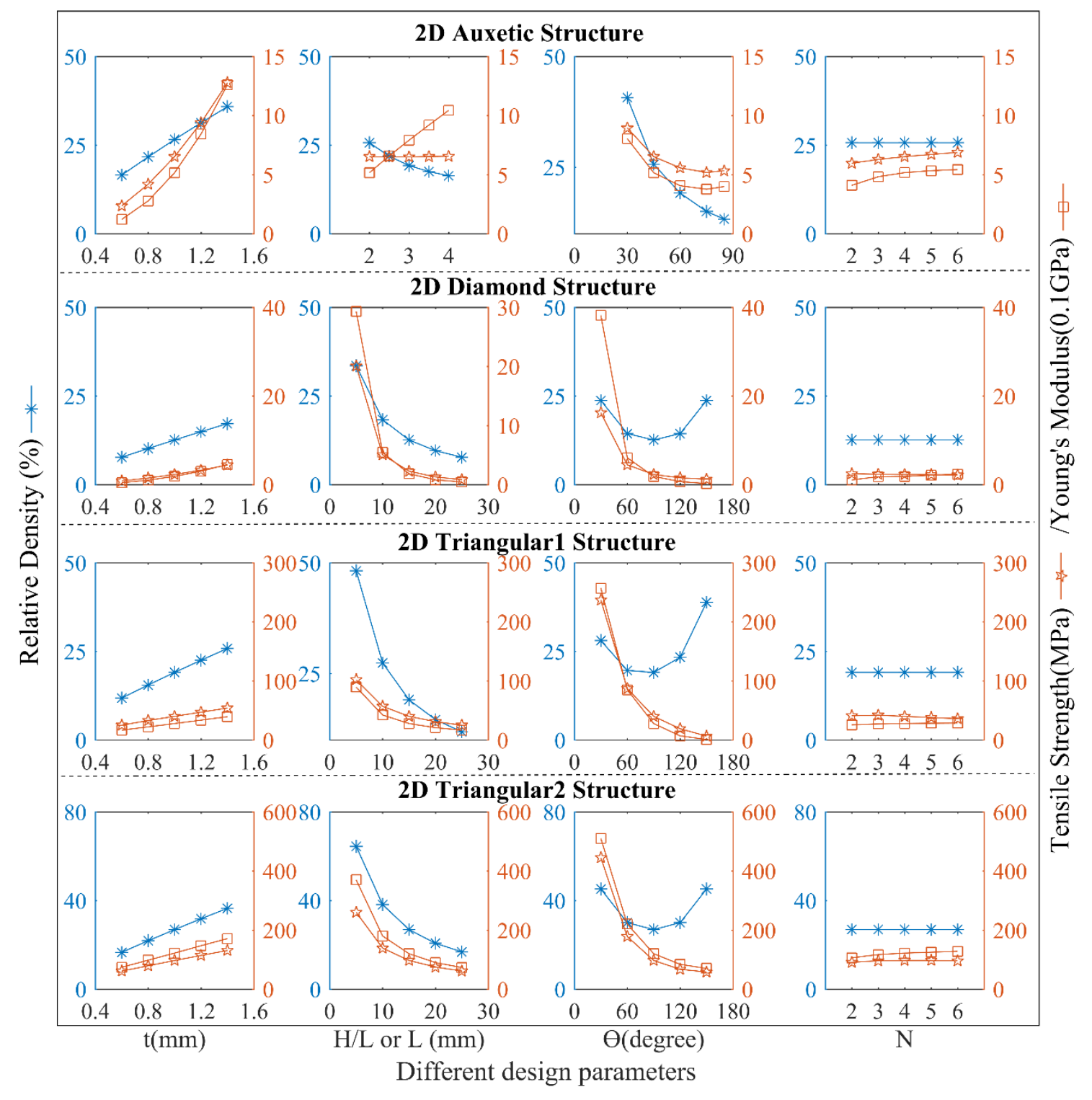

Fig. 3. 3 Tensile strength/Young's modulus versus design parameters of four types of cellular structures

Fig. 3.3 shows the relationships between initial tensile strength/Young's modulus and different geometry parameters of four types of cellular structures. It is seen that both tensile strength and Young's modulus are highly dependent on the geometry designs. According to the classic theory for cellular structures [41], the modulus and strength of the 
open cell foam structures are predominantly determined by the relative density $\rho_{r}$ as follows:

$$
\begin{gathered}
\frac{E}{E_{s}}=C_{1} \rho_{r}^{2} \\
\frac{\sigma}{\sigma_{s}}=C_{2} \rho_{r}^{3 / 2}
\end{gathered}
$$

where $E, \sigma$ and $\rho_{r}$ are the Young's modulus, strength and relative density of the foam and $E_{s}$ and $\sigma_{s}$ are the Young's modulus and strength of the solid cell wall material. $C_{1}$ and $C_{2}$ are constants related to the cell geometry $\left(C_{l}=1\right.$ and $C_{2}=0.3$ for a wide variety of foams). From the results shown in Fig. 3.3, it can be generally concluded that an increase/decrease in relative density through the wall length $\mathrm{L}$ or wall thickness $t$ results in a corresponding increases/decreases in tensile strength and Young's modulus, which agrees with the general trends as shown in Equation (3.1)-(3.2) [41]. However, for the 2D auxetic structures, the tensile strength does not exhibit significant dependency on the relative density as the $H / L$ ratio varies, while the Young's modulus even exhibited an increasing trend with decreasing relative density. Such seemingly counterintuitive observation is closely associated with the geometry design schemes used. In this study, the change of $H / L$ ratio is achieved by varying $H$ with a fixed $L$ value. Generally larger $H$ corresponds to more significant bending effects of the vertical walls, and these effects would cause larger bending deformation of the reentrant walls, which further results in a larger principal stress and a larger strength. However, with increasing $\mathrm{H}$, while the vertical walls towards the boundaries exhibit more significant bending deformation, such effect is much less pronounced for the vertical walls in the internal areas. That is to say, the walls towards the center inner area are less affected by $H$. Since the critical walls of the $4 \times 4$ auxetic structures are located towards the center 
areas of the structure, the initial failure strength becomes almost independent of the length of $H$. On the other hand, when the length of $H$ becomes larger, the overall strain becomes smaller with the same displacement. This would result in a larger Young's modulus. Such geometry-specific "negative" relative density-property relationship could also be observed for 2D diamond, 2D trianglular1 and 2D triangular2 structures, for which with the opening angle increases from $90^{\circ}$ to $150^{\circ}$ the Young's modulus and tensile strength exhibit decreasing trends when the relative density increases. This is because when the opening angle increases, the oblique walls are subjected to a larger bending moment, which makes the structure weaker.

It is worth noting that the sensitivities of the tensile strength and Young's modulus to the relative density are different among these four structures. Based on the results from Fig. 3.3, the relationship (the power number and the constant $C_{1}$ and $C_{2}$ ) between the tensile strength/ Young's modulus and relative density can be obtained using least square regression fitting, which are shown in Table 3.2. In Table 3.2, $\rho_{r-t}, \rho_{r-L}, \rho_{r-\theta}$ represent the relative densities as a function of the wall thickness, wall length and opening angle respectively. Comparing these results, it could be readily verified that the stretchingdominated structures, including the 2D triangular 1 and $2 \mathrm{D}$ triangular2 structures, exhibit relatively smaller sensitivities (smaller power numbers) to the relative densities compared with the bending-dominated structures, which include the 2D auxetic and 2D diamond structures. Furthermore, it can be seen that these four structures exhibit different relative density sensitivity characteristics when specific design parameters are varied. For the 2D diamond, 2D triangular1 and 2D triangular2 structure, similar relative density sensitivities were observed when the thickness and wall length vary. In contrast, for the $2 \mathrm{D}$ auxetic 
structure, the Young's modulus exhibits an inversed dependency on the relative density when the opening angle varies. Besides, the tensile strength and Young's modulus of 2D auxetic structure exhibit different relative density sensitivities when the wall thickness or the wall length varies. Such varying relative density-property relationships for the same type of unit cell designs were not previously observed, which is again likely associated with the specific geometry variation rules employed in this study and warrants future follow-up investigations.

Table 3. 2 The Young's modulus and tensile strength versus relative density

\begin{tabular}{|c|c|c|c|c|}
\hline & 2D Auxetic & 2D Diamond & 2D Triangular1 & 2D Triangular2 \\
\hline \multirow{2}{*}{$\begin{array}{c}\text { Thickness } \\
(t)\end{array}$} & $\frac{E}{E_{s}}=0.0022 \rho_{r-t}^{3}$ & $\frac{E}{E_{s}}=0.780 \rho_{r-t}^{3}$ & $\frac{E}{E_{s}}=0.143 \rho_{r-t}$ & $\frac{E}{E_{s}}=0.434 \rho_{r-t}$ \\
\cline { 2 - 5 } & $\frac{\sigma}{\sigma_{s}}=0.098 \rho_{r-t}^{2}$ & $\frac{\sigma}{\sigma_{s}}=0.144 \rho_{r-t}^{2}$ & $\frac{\sigma}{\sigma_{s}}=0.196 \rho_{r-t}$ & $\frac{\sigma}{\sigma_{s}}=0.336 \rho_{r-t}$ \\
\hline $\begin{array}{c}\text { Wall } \\
\text { length } \\
(L)\end{array}$ & $\frac{E}{E_{s}}=0.0006 \rho_{r-L}^{-3 / 2}$ & $\frac{E}{E_{s}}=0.675 \rho_{r-L}^{3}$ & $\frac{E}{E_{s}}=0.177 \rho_{r-L}$ & $\frac{E}{E_{s}}=0.555 \rho_{r-L}$ \\
\cline { 2 - 5 } & $N A$ & $\frac{\sigma}{\sigma_{s}}=0.173 \rho_{r-L}^{2}$ & $\frac{\sigma}{\sigma_{s}}=0.202 \rho_{r-L}$ & $\frac{\sigma}{\sigma_{s}}=0.397 \rho_{r-L}$ \\
\hline $\begin{array}{c}\text { Opening } \\
\text { angle } \\
\theta)\end{array}$ & $\frac{E}{E_{s}}=0.0137 \rho_{r-\theta}^{1 / 2}$ & $\mathrm{NA}$ & $\mathrm{NA}$ & $\mathrm{NA}$ \\
\cline { 2 - 5 } & $\frac{\sigma}{\sigma_{s}}=0.0132 \rho_{r-\theta}^{1 / 2}$ & $\mathrm{NA}$ & $\mathrm{NA}$ & $\mathrm{NA}$ \\
\hline
\end{tabular}

3.2.2.2 Size and topology effects on strength and modulus during tensile failure

From Fig. 3.3, it is clear that the modulus and initial tensile strength of the stretching-dominated/ higher nodal connectivity cellular structures are much higher than those of the bending-dominated/ lower nodal connectivity cellular structures of the same relative densities. However, after the initial failure of the cellular structure, different structures exhibit different responses during the tensile failure. Fig. 3.4 further illustrates 
the stepwise retaining Young's modulus and tensile strength when the two bendingdominated structures gradually fail. In Fig. 3.4, S1, S2, S3 and S4 indicate the steps during the failure, which also correspond to the different peaks in the stress-strain curve shown in Fig. 3.2. The tensile strength ratio and Young's modulus ratio represent the ratio of the tensile strength and Young's modulus of different steps to their respective initial values. The values under each design parameter category are the averaged values across all the levels of that particular design parameter. It is obvious that both structures exhibit certain levels of retaining strength and modulus of the original structure during the stepwise failures. In comparison, the 2D diamond structures exhibit slower strength and modulus degradation compared with the 2D auxetic structures as the fracture progresses. Besides, it can be seen that for both the strength and modulus of both structures, the stepwise property degradation rate is relatively consistent for different geometry designs. Based on the results of Fig. 3.4, Table 3.3 shows the overall scaling relationship averaged over all different design parameters, which further demonstrates the overall property degradation characteristics of the two bending-dominated designs. 
(a)

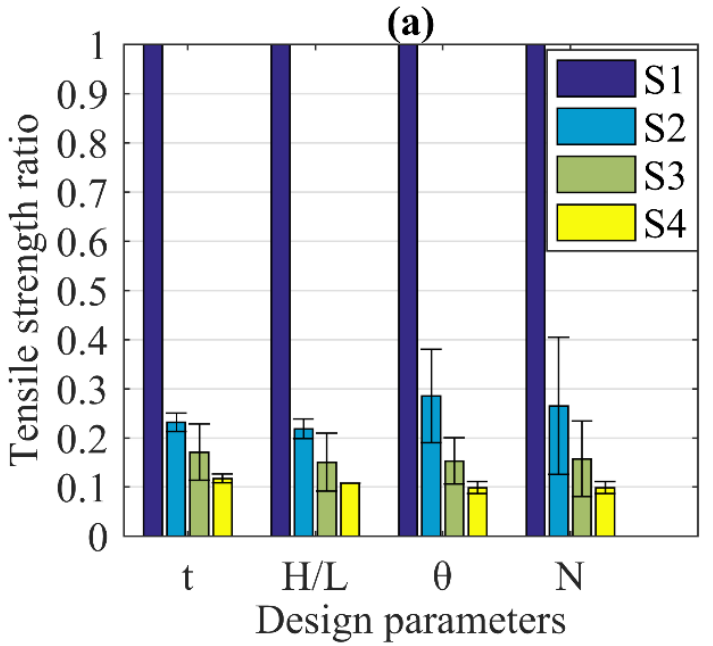

(c)

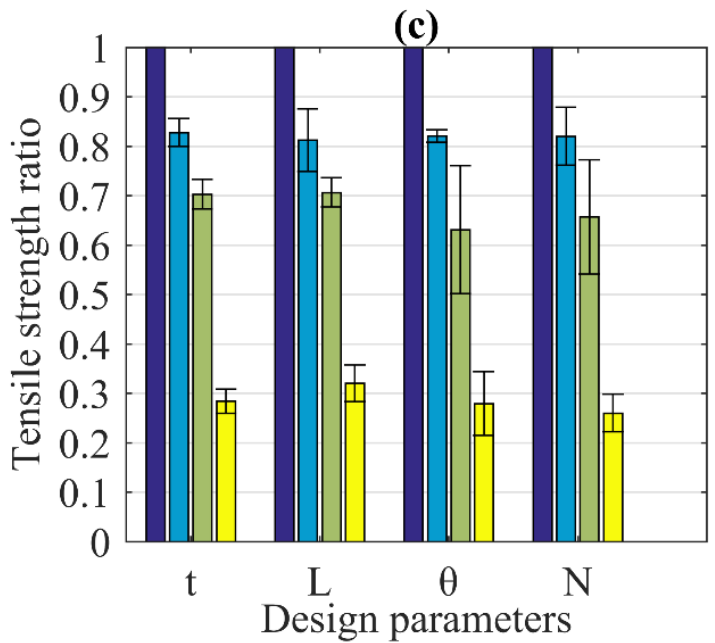

(b)

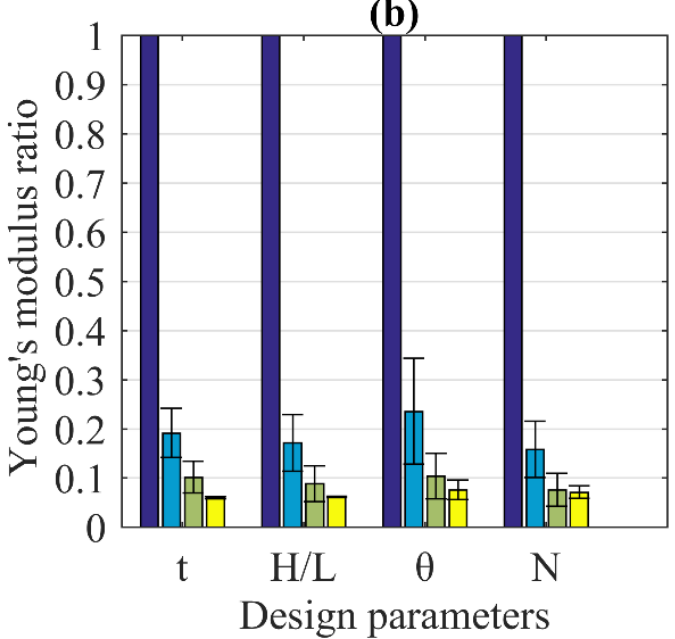

(d)

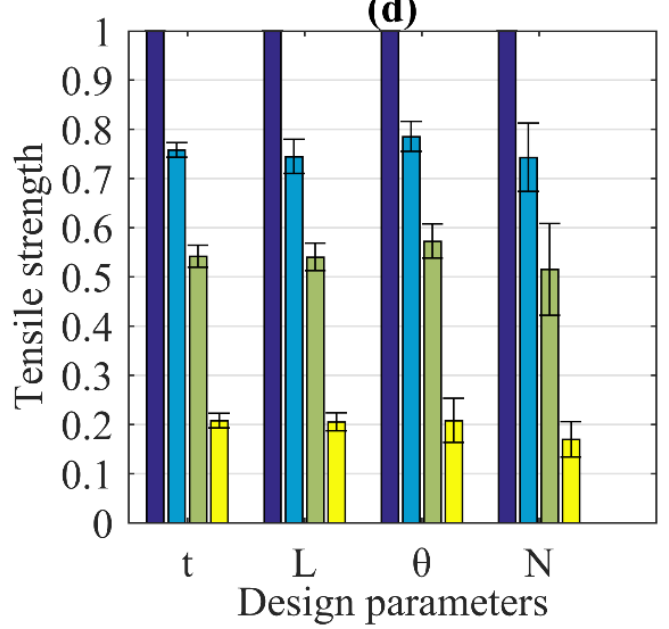

Fig. 3. 4 Averaged stepwise failure characteristics for different design parameters:

Stepwise tensile strength (a) and stepwise Young's modulus (b) analysis of 2D auxetic structures; Stepwise tensile strength (c) and stepwise Young's modulus (d) analysis of 2D diamond structures

Table 3. 3 Scaling relationships among the stepwise Young's modulus and tensile strength for two bending-dominated structures

\begin{tabular}{|c|c|c|c|}
\hline \multicolumn{2}{|c|}{ 2D Auxetic } & \multicolumn{2}{|c|}{ 2D Diamond } \\
\hline$\sigma_{s 2}=0.25 \sigma_{s 1}$ & $E_{s 2}=0.19 E_{s 1}$ & $\sigma_{s 2}=0.82 \sigma_{s 1}$ & $E_{s 2}=0.76 E_{s 1}$ \\
\hline
\end{tabular}




$$
\begin{array}{llll}
\sigma_{s 3}=0.16 \sigma_{s 1} & E_{s 3}=0.09 E_{s 1} & \sigma_{s 3}=0.67 \sigma_{s 1} & E_{s 3}=0.54 E_{s 1} \\
\sigma_{s 4}=0.11 \sigma_{s 1} & E_{s 4}=0.06 E_{s 1} & \sigma_{s 4}=0.32 \sigma_{s 1} & E_{s 4}=0.20 E_{s 1}
\end{array}
$$

3.2.3 Size and topology effects on tensile failure responses

\subsubsection{Size and topology effects on energy absorption}

For solid materials, their fracture properties are often characterized by the total amount of energy that is dissipated during the fracture propagation/failure process. Therefore, in this study the energy absorptions of the cellular structures were also investigated. In order to account for the effect of relative density, the normalized energy absorption was also employed for analysis, which is the total energy absorption of the structure divided by its relative density. A comparison of the energy absorption and normalized energy absorption of four types of cellular structures are shown in Fig. 3.5. Generally, the overall energy absorption of a cellular structure increases with higher relative density, which agrees with the classic theory. The energy absorption of the 2D auxetic and 2D diamond structure follow the trend of the relative density. When the opening angle varies from $30^{\circ}$ to $90^{\circ}$, the energy absorption decreases, while it increases when the opening angle varies from $90^{\circ}$ to $150^{\circ}$. However, for the $2 \mathrm{D}$ triangular 1 and $2 \mathrm{D}$ triangular2 structure, the energy absorption keeps decreasing when the opening angle increases from $90^{\circ}$ to $150^{\circ}$. These can be also observed from Fig. 3.2. The area under the stress-strain curves (volumetric energy absorption) for the 2D triangular1 and 2D triangular2 structure continue to decrease when the opening angle increases from $30^{\circ}$ to $150^{\circ}$. 


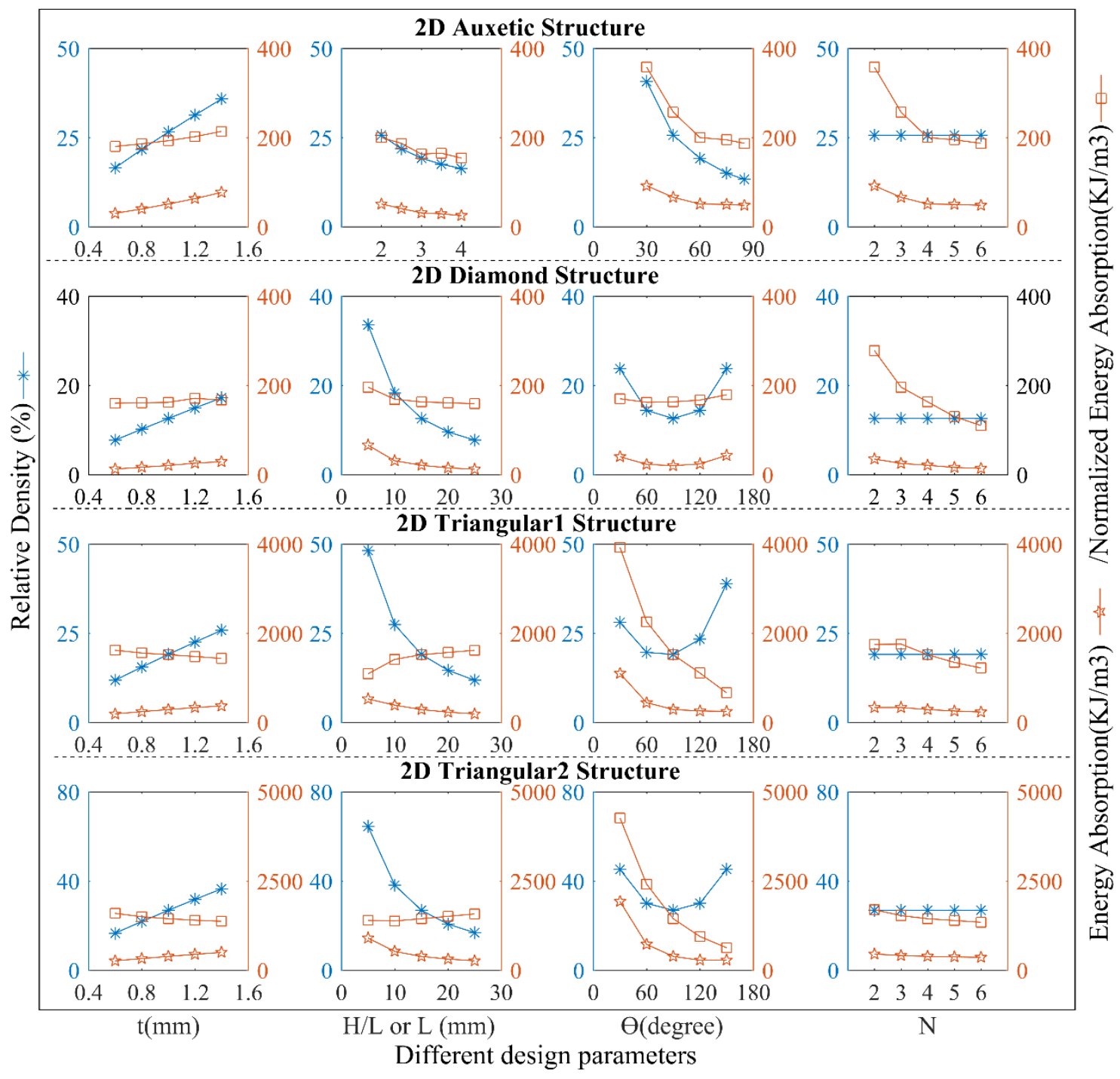

Fig. 3. 5 Energy absorption/ Normalized energy absorption versus design parameters of four types of cellular structures

For the normalized energy absorption of these four structures, the two bendingdominated structure, 2D auxetic and 2D diamond with lower nodal connectivity (3 and 4), exhibit the same trend with the relative density when wall thickness, wall length or opening angle changes. This also indicates that the energy absorption efficiency of the bendingdominated structures is mostly attributed by the unit mass of the structure. However, for the two stretching-dominated structures, 2D triangular1 and 2D triangular2 with higher 
nodal connectivity (6 and 8), the normalized energy absorption decreases when the relative density increases. In another word, even though these two structures can absorb more total energy, the energy absorption efficiency actually decreases with more material.

For the size effect on the energy absorption and the normalized energy absorption of these four structures, it is clear from Fig. 3.5 that both characteristics decrease when the unit cell number increases, although the relative densities remain unchanged. For the 2D auxetic structure and 2D triangular2 structure, energy absorption characteristics appear to stabilize when the unit cell number is bigger than 4 , while for the 2D diamond structure and 2D triangular1 structure such trend appears less apparent. On the other hand, both the 2D auxetic and 2D diamond structures exhibit more significant size effect of energy absorption at smaller cell number sizes. This can be explained with the help of Fig. 3.2. For cellular materials, the energy absorption ability can be considered to be largely in proportion to the structural strength [24]. From the stress-strain curve shown in Fig. 3.2, it is clear that the unit cell numbers have little effects on the initial strength of the four structures investigated. As previously discussed, the stretching-dominated structures with higher nodal connectivity exhibit only one stress peaks. Therefore, for these structures the energy absorption ability is highly depended on the initial failure strength. On the other hand, for the bending-dominated structures with lower nodal connectivity, the energy absorption ability is also influenced by the consequent stress peaks after the first one.

\subsubsection{Size and topology effects on tensile failure pattern}

Fig. 3.6 to Fig. 3.9 show the failure propagation patterns of the four types of cellular structures with different geometry design parameters (note that the length and angle of the structures illustrated in the figures do not reflect the actual designs). Due to the symmetry 
of these 2D cellular structures, there would be four identical walls (graphically, upper left, upper right, lower left and lower right) having the maximum stress theoretically. Without losing generality, in this paper the wall in the upper left of the structure was selected as the failure initiation. In these figures, the numbers indicate the failure sequences. For example, for the auxetic structures with wall thickness of $t=0.6 \mathrm{~mm}$ (upper-left image in Fig. 3.6), the fracture will initiate from the wall located at upper left as discussed above. Based on the model, this failure initiation will cause the simultaneous fracture of another four walls which are also labeled number 1 , while consequent fracture events occur by only one wall at each step. From these figures, it could also be seen that for the two bending-dominated structures, the failure patterns exhibit more progressive characteristics. On the other hand, the failure propagation of the stretching-dominated structures appears to be rather catastrophic. Once the first wall fails, large numbers of walls may fracture immediately. Such observations also resonant with the previous discussions about the stress-strain characteristics with these structures.

Fig. 3.6 shows the failure propagation characteristics of different 2D auxetic structures. It can be seen that the failure propagation does not appear to be significantly dependent on the wall thickness, $\mathrm{H} / \mathrm{L}$ ratio or unit cell numbers. On the other hand, the crack propagation plane appears to exhibit some dependency on the opening angle. It is noted that the crack plane transits from the middle layers towards the boundary layers as the opening angle increases from $30^{\circ}$ to $85^{\circ}$. Moreover, the results indicate that the failure characteristics of the two auxetic structures exhibit minimum size effect. 

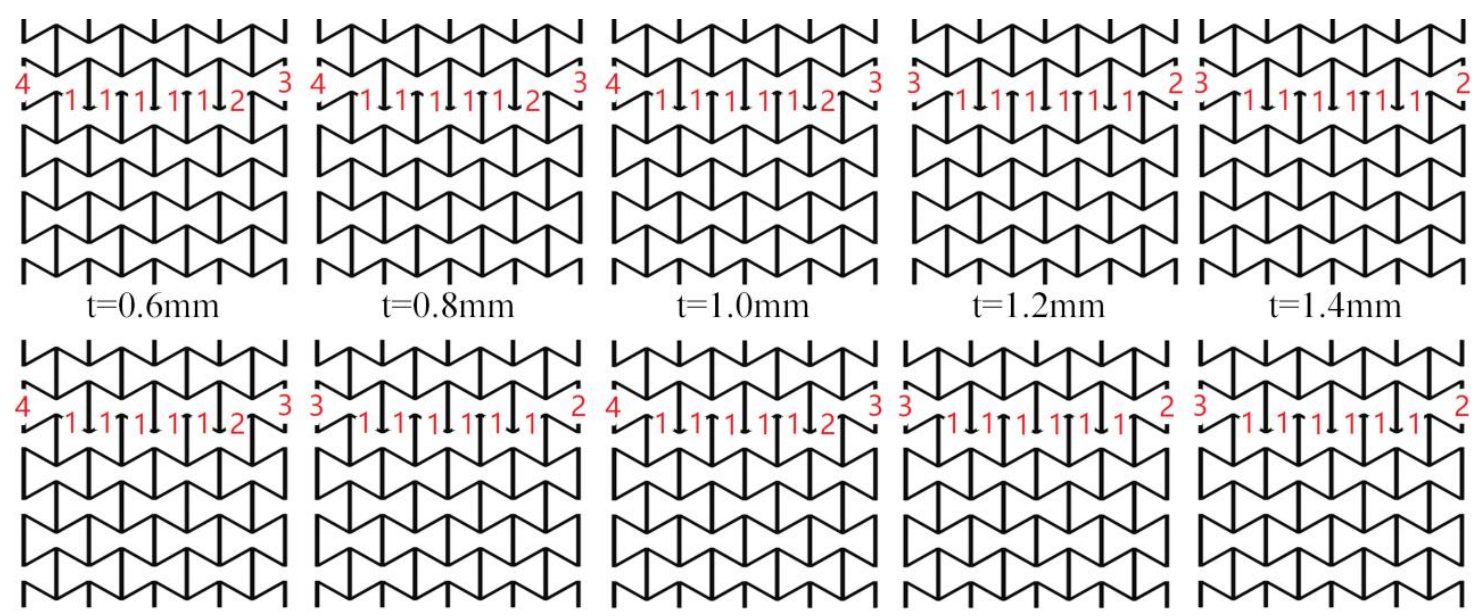

$\mathrm{t}=0.8 \mathrm{~mm}$

$\mathrm{t}=1.0 \mathrm{~mm}$

$\mathrm{t}=1.2 \mathrm{~mm}$

$\mathrm{t}=1.4 \mathrm{~mm}$

$\mathrm{H} / \mathrm{L}=2$
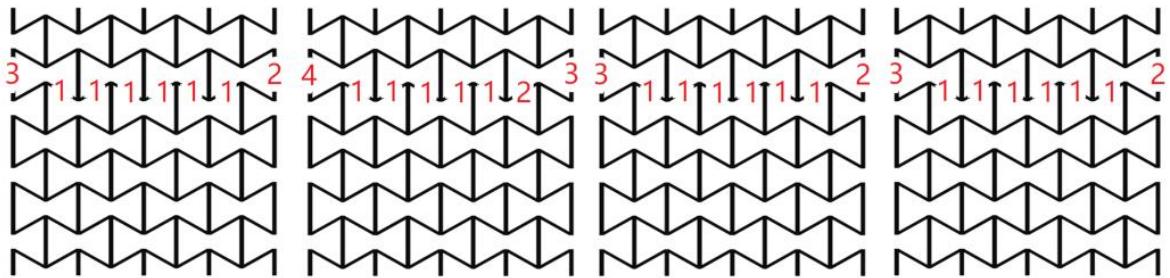

NTN

3 C

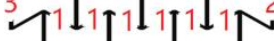

Nom

NOIN

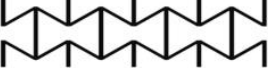

$\theta=30^{\circ}$

$\mathrm{H} / \mathrm{L}=2.5$

$\mathrm{H} / \mathrm{L}=3$

$\mathrm{H} / \mathrm{L}=3.5$

$\mathrm{H} / \mathrm{L}=4$
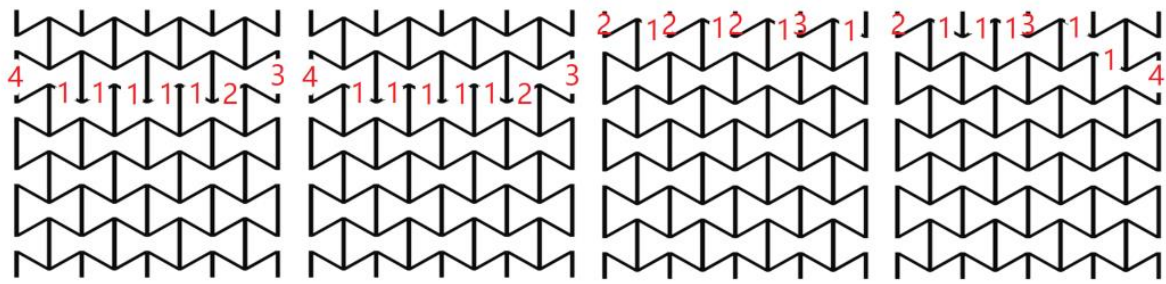

$\mathrm{O}=45^{\circ}$

$\theta=60^{\circ}$

$\mathrm{O}=75^{\circ}$

$\theta=85^{\circ}$

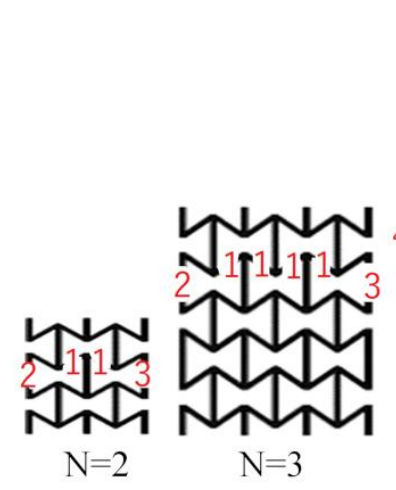

2

Nove

$\Theta=60$
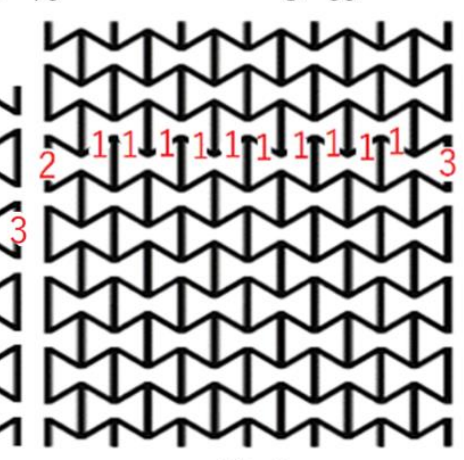

$\mathrm{N}=5$

$\mathrm{N}=6$

Fig. 3.6 The tensile failure patterns under different design parameters of 2D auxetic structure

Fig. 3.7 shows the failure propagation patterns of the 2D diamond structures. The 2D diamond structures exhibit consistent diagonal failure patterns regardless of the geometry designs, which might indicate that the effect of the boundary constraints is predominant for this type of structure. However, it is notable from Fig. 9 that the tensile failure pattern of the structure with wall length of $5 \mathrm{~mm}$ was quite different from the others. 
The relative density of this structure is $33.56 \%$, which is the largest among all the design variations. Therefore, further relative density analysis was conducted by further extending the wall length and wall thickness towards higher relative density levels. It was found that when the wall length becomes smaller than $5 \mathrm{~mm}$ (with a relative density $>33.56 \%$ ) or the wall thickness becomes bigger than $2.7 \mathrm{~mm}$ (with a relative density $>30.74 \%$ ), the $2 \mathrm{D}$ diamond structures will exhibit the same V-shaped failure pattern. Therefore, it was concluded that for the 2D diamond structures with varying relative density, there exist two distinctive failure patterns. 


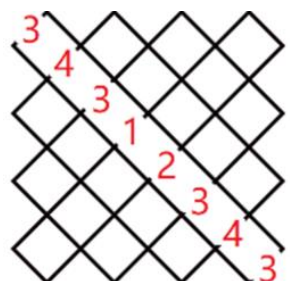
$\mathrm{t}=0.6 \mathrm{~mm}$

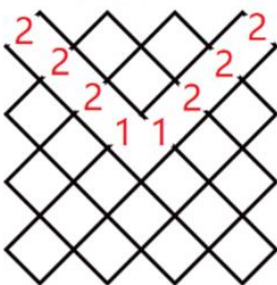

$\mathrm{L}=5 \mathrm{~mm}$

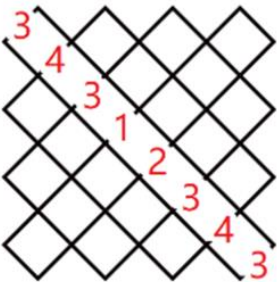

$\Theta=30^{\circ}$

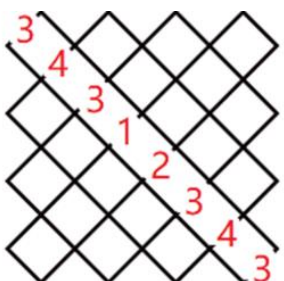

$\mathrm{t}=0.8 \mathrm{~mm}$

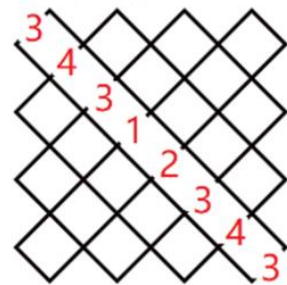

$\mathrm{L}=10 \mathrm{~mm}$

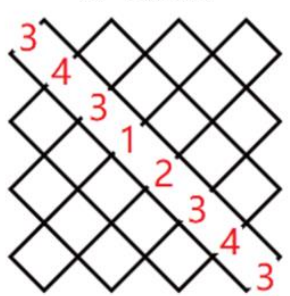

$\Theta=60^{\circ}$

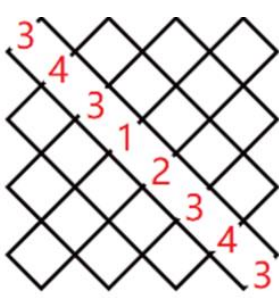

$\mathrm{t}=1.0 \mathrm{~mm}$

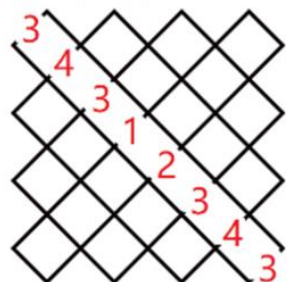

$\mathrm{L}=15 \mathrm{~mm}$

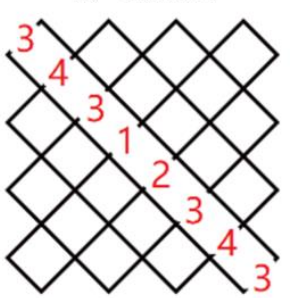

$\theta=90^{\circ}$

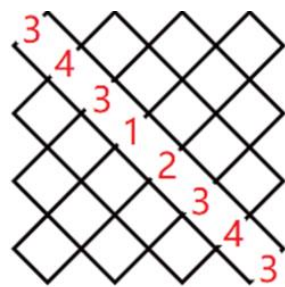

$\mathrm{t}=1.2 \mathrm{~mm}$

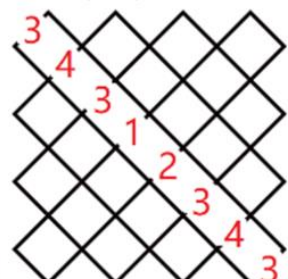

$\mathrm{L}=20 \mathrm{~mm}$

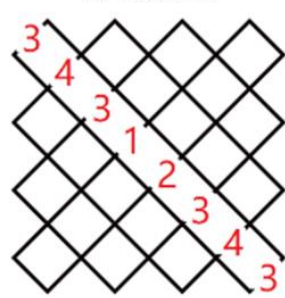

$\Theta=120^{\circ}$

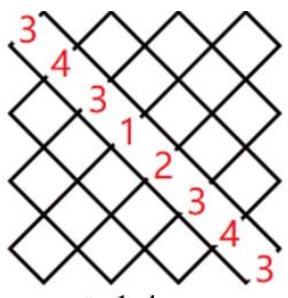

$\mathrm{t}=1.4 \mathrm{~mm}$

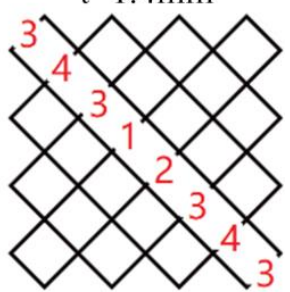

$\mathrm{L}=25 \mathrm{~mm}$

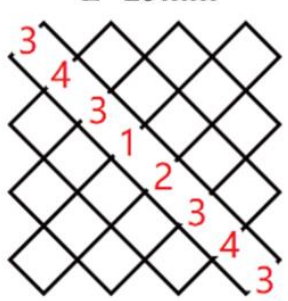

$\Theta=150^{\circ}$

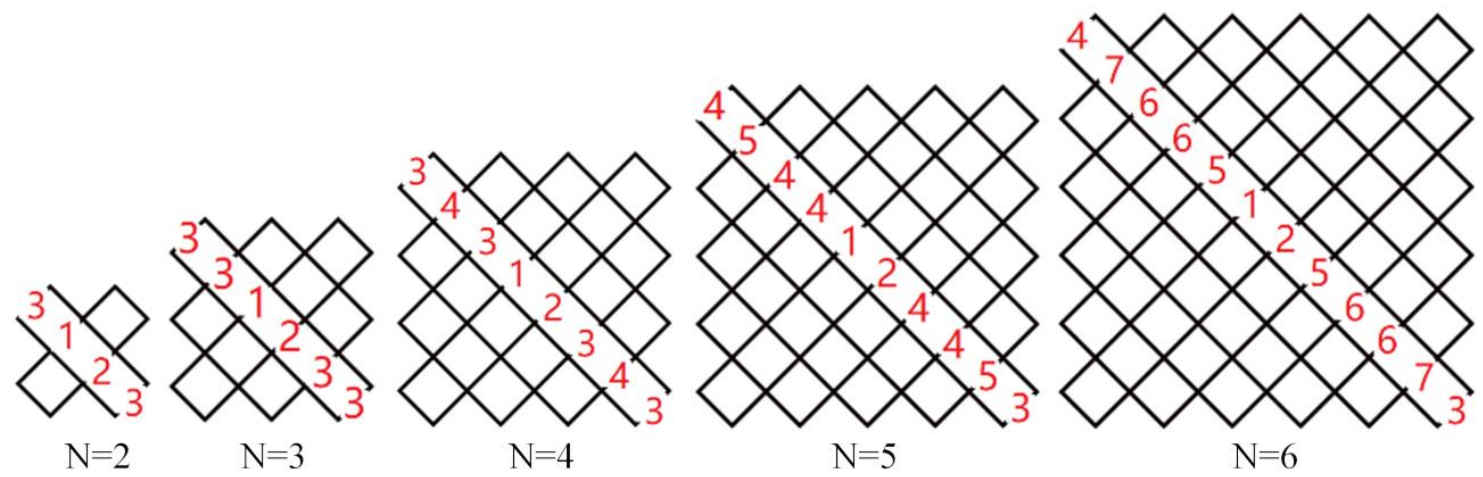

Fig. 3. 7 The tensile failure patterns under different design parameters of $2 \mathrm{D}$ diamond structure

Fig. 3.8 shows the failure propagation patterns of the 2D triangular1 structure. In general, the crack initiation and propagation of this type of structure appears to concentrate on the boundary areas where the stress concentration effects are the strongest. The crack pattern appears to transit from the interiors of the structures towards the boundaries as the opening angle increases from $30^{\circ}$ to $150^{\circ}$, when the wall thickness increases, or when the 
wall length reduces. On the other hand, the effect of number of unit cells in the pattern appears to be relatively insignificant, especially when the unit cell number is larger than 4 . It was speculated that for this type of geometry the lower-relative density structures exhibit a boundary failure pattern while the higher-relative density structures exhibit an interior failure pattern.

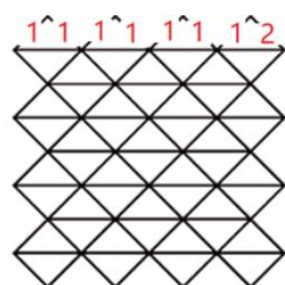

$\mathrm{t}=0.6 \mathrm{~mm}$

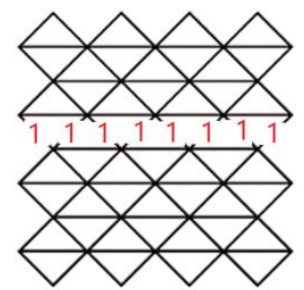

$\mathrm{L}=5 \mathrm{~mm}$

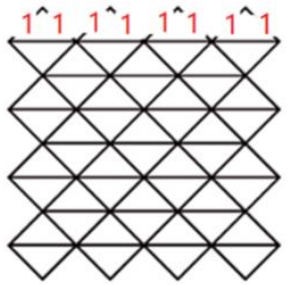

$\mathrm{O}=30^{\circ}$

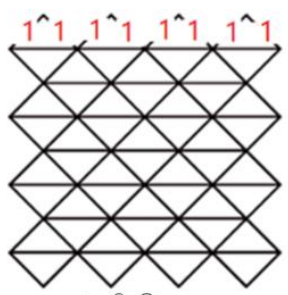

$\mathrm{t}=0.8 \mathrm{~mm}$

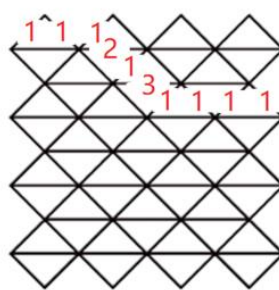

$\mathrm{L}=10 \mathrm{~mm}$

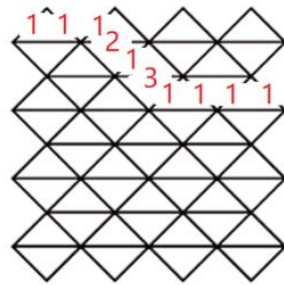

$\mathrm{O}=60^{\circ}$

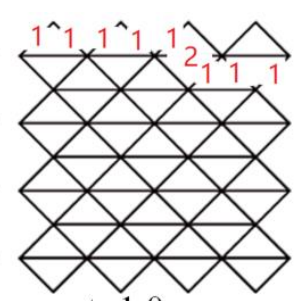

$\mathrm{t}=1.0 \mathrm{~mm}$

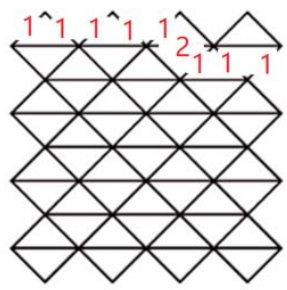

$\mathrm{L}=15 \mathrm{~mm}$

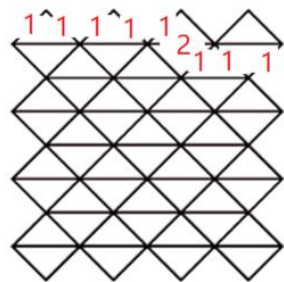

$\mathrm{O}=90^{\circ}$

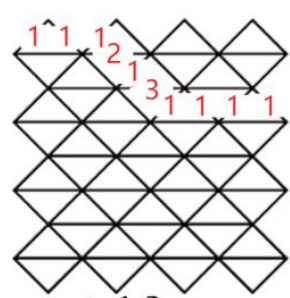

$\mathrm{t}=1.2 \mathrm{~mm}$

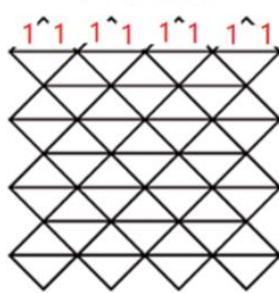

$\mathrm{L}=20 \mathrm{~mm}$

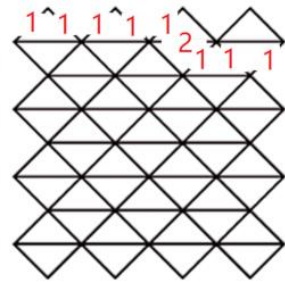

$\mathrm{O}=120^{\circ}$

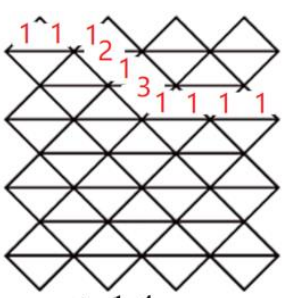

$\mathrm{t}=1.4 \mathrm{~mm}$

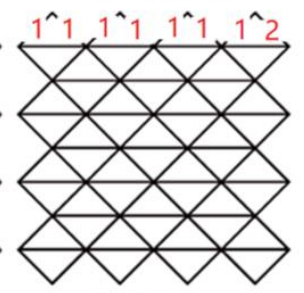

$\mathrm{L}=25 \mathrm{~mm}$

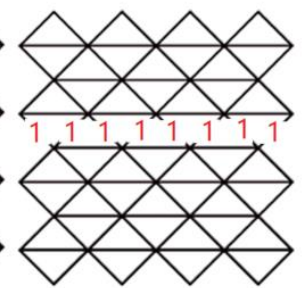

$\theta=150^{\circ}$

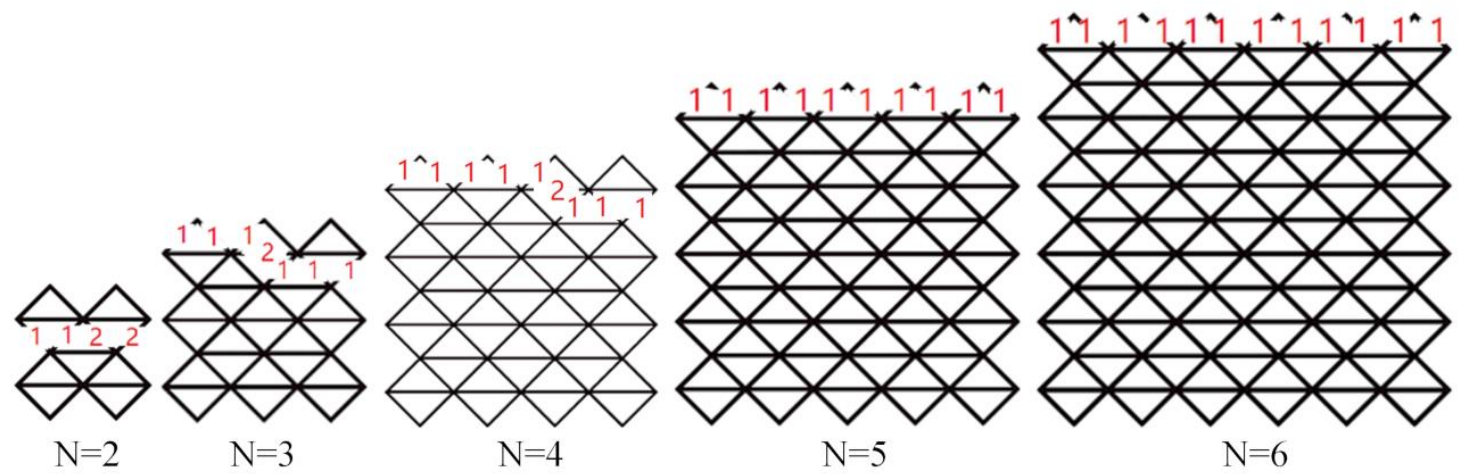

Fig. 3. 8 The tensile failure patterns under different design parameters of 2D triangular1 structure 
Lastly, Fig. 3.9 shows the failure propagation patterns of the 2D triangular2 structure. Unlike the 2D triangular1 structures, the 2D triangular2 structures exhibits rather consistent fracture patterns under most design conditions. It also appears that for the design variations that have higher relative densities, the failure pattern is rather catastrophic.

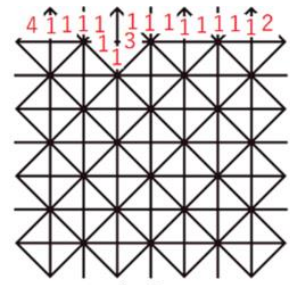

$\mathrm{t}=0.6 \mathrm{~mm}$

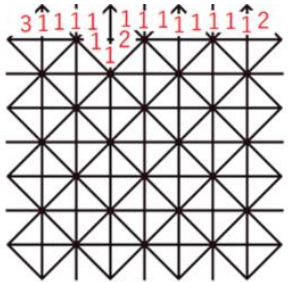

$\mathrm{t}=0.8 \mathrm{~mm}$

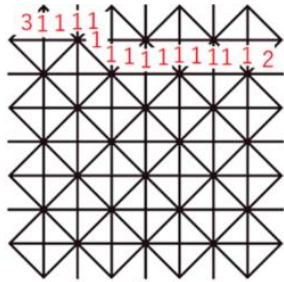

$\mathrm{t}=1.0 \mathrm{~mm}$

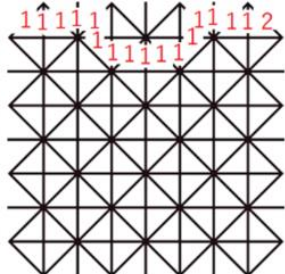

$\mathrm{t}=1.2 \mathrm{~mm}$
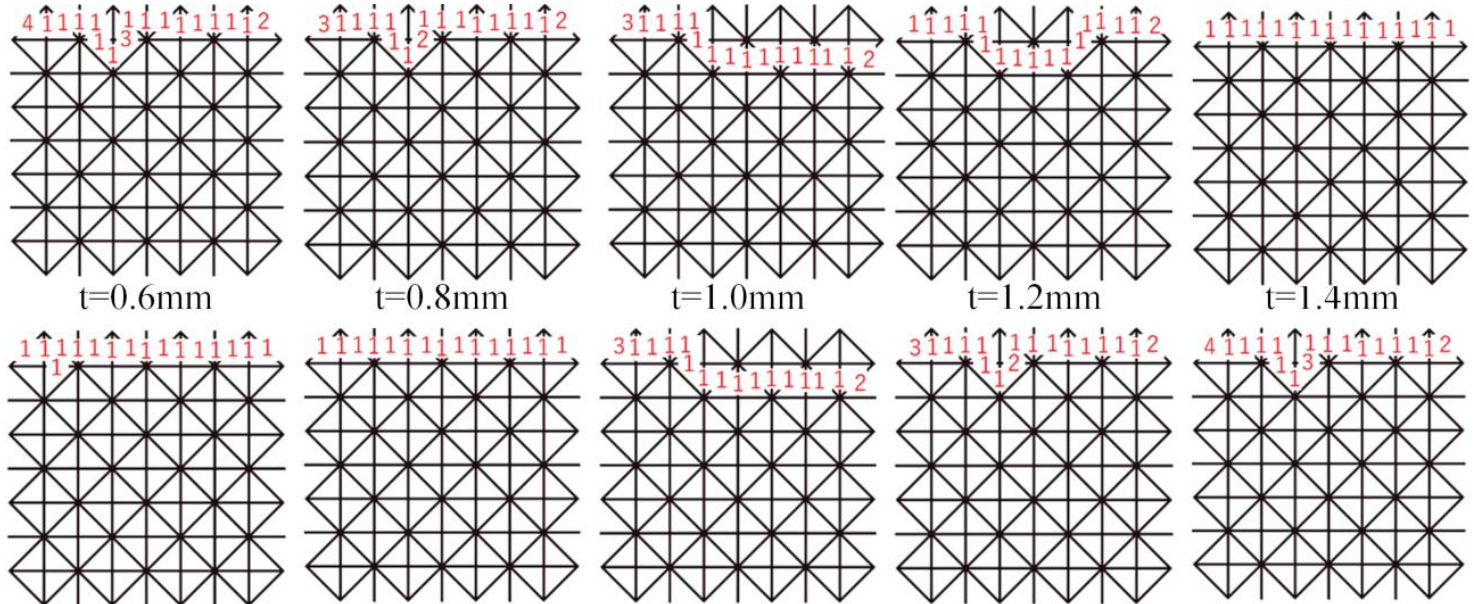

$\mathrm{L}=5 \mathrm{~mm}$

$\mathrm{L}=10 \mathrm{~mm}$

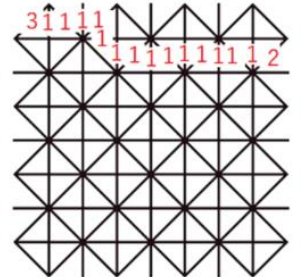

$\mathrm{L}=15 \mathrm{~mm}$

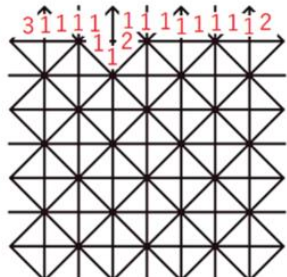

$\mathrm{L}=20 \mathrm{~mm}$

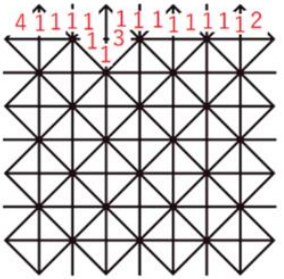

$\mathrm{L}=25 \mathrm{~mm}$

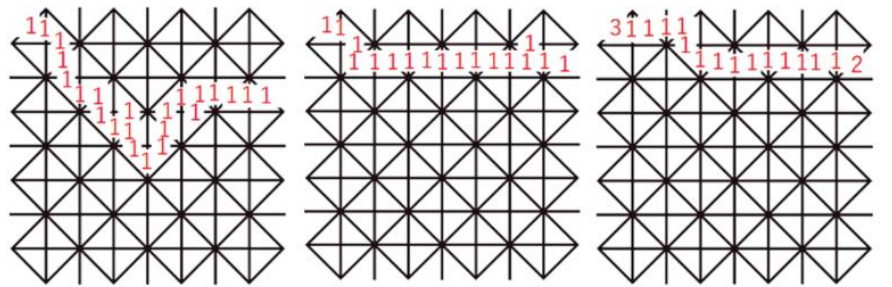

$\mathrm{O}=30^{\circ}$

$\mathrm{O}=60^{\circ}$

$\mathrm{O}=90^{\circ}$
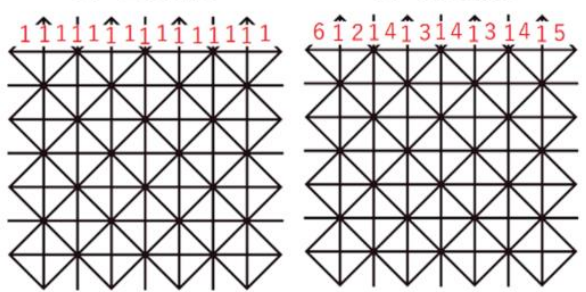

$\mathrm{O}=120^{\circ}$

$\mathrm{O}=150^{\circ}$

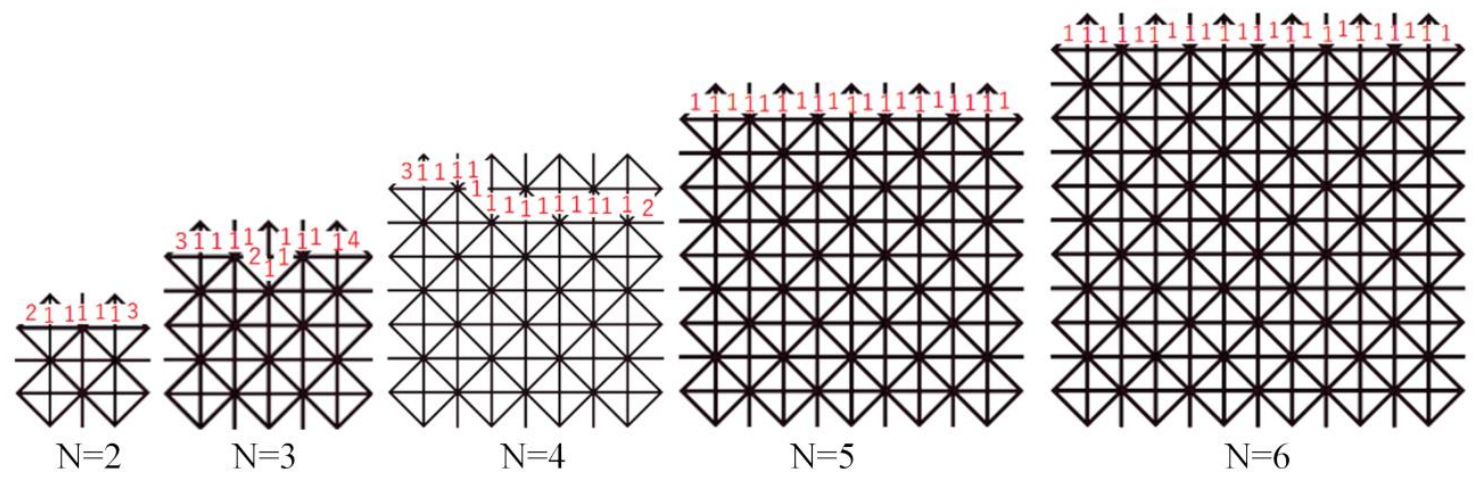

Fig. 3.9 The tensile failure patterns under different design parameters of 2D triangular2 structure 


\subsubsection{Failure mode analysis}

From the analysis in Section 3.3.3, the 2D diamond structures and 2D triangular1 structures exhibit relative density-dependent failure patterns, while the $2 \mathrm{D}$ auxetic structures and 2D triangular2 structures exhibit more consistent failure patterns. This was further investigated through the analysis the initial failure locations with these designs.

For the auxetic structure, the diamond structure and the triangular2 structure, the initial failure locations remain largely consistent when the design parameters vary, as are indicated by the index "1" from Fig. 3.6, Fig. 3.7 and Fig. 3.9. For the auxetic structure and diamond structure, the failure initiations are located in the middle of the structure, while for the triangular2 structure, the failure initiation occurs at the corner of the structure. On the other hand, for the triangular1 structure, the failure initiation occurs either from the center or from the corner of the structures, as shown in Fig. 3.8. In order to investigate the boundary effect on the failure initiation, the normalized principal stresses of wall A (located at the corner of the structure) and wall B (located in the middle of the structure) (shown in Fig. 3.10(a-d)) were analyzed under same applied strain for each structure as their respective wall length $(\mathrm{L}) /$ height and length ratio (H/L) vary. The normalized principal stress was obtained by dividing the principal stress in the wall by the principal stress from the corresponding ideal structure that has infinite patterns (shown in Fig. 3.10eh), which have no boundary and size effect. 


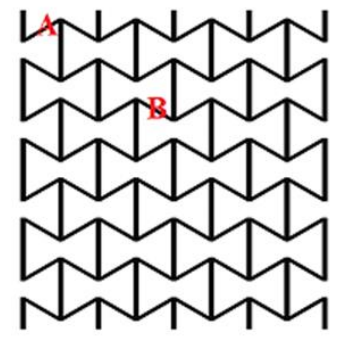

(a)

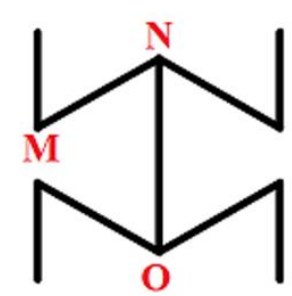

(e)

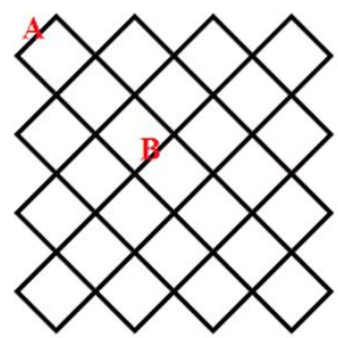

(b)

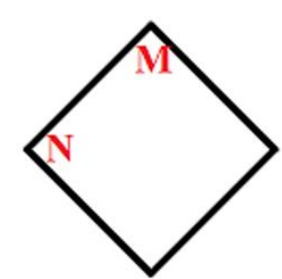

(f)

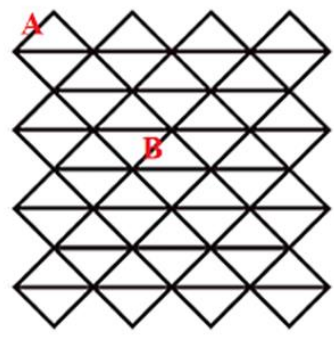

(c)

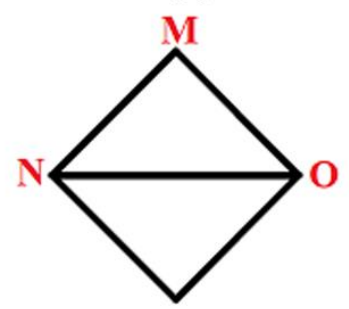

(g)

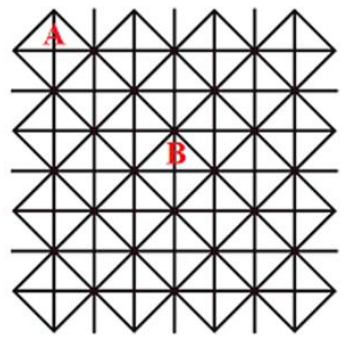

(d)

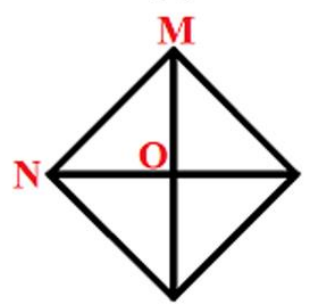

(h)

Fig. 3.10 The boundary wall A and middle wall B for (a) 2D auxetic, (b) 2D diamond, (c) 2D triangular1 and (d) 2D triangular2 structure and the corresponding ideal structure unit cell: (e) 2D auxetic, (f) 2D diamond, (g) 2D triangular1 and (h) 2D triangular2

Fig. 3.11 showed the comparison of the normalized principal stress levels of wall A and wall B for each of the four types of unit cell designs. It is clear that the normalized principal stress in wall $\mathrm{B}$ is much higher than that in wall $\mathrm{A}$ for the $2 \mathrm{D}$ auxetic structure (Fig. 3.11(a)) and 2D diamond structure (Fig. 3.11(b)). In contrast, the normalized principal stress in wall $\mathrm{B}$ is much lower than that in wall A for the $2 \mathrm{D}$ triangular2 structure (Fig. 3.11(d)). This further illustrates the previously observed failure patterns with these structures, in which the failure initiated from the center of structure for the $2 \mathrm{D}$ auxetic structure (Fig. 3.6) and the 2D diamond structure (Fig. 3.7), and from the corner for the 2D triangular2 structure (Fig. 3.9). On the other hand, for the 2D triangular1 structure (Fig. 3.8), the normalized principal stress is larger in wall B when the wall length is short. As the wall length increases, the normalized principal stress became larger in wall A, which 
becomes dominant when the wall length is larger than $6 \mathrm{~mm}$ in the case of Fig. 3.11c. This provides an explanation for the failure pattern mode change illustrated in Fig. 3.8.

Besides, from Fig. 3.11, we can see that the normalized principal stress levels of the two types of walls are less than unity for both the 2D auxetic and 2D triangular2 structure, which suggests that the finite boundary effects result in enhanced strengths with both type of structures. For the ideal infinite 2D auxetic and 2D triangular2 structures, the vertical walls (wall NO for 2D auxetic and wall MO for 2D triangular2 in Fig. 3.10) are not subjected to any bending moment or shear force due to the symmetry of the structures. However, when considering the finite boundary effect, these vertical walls will be subjected to both types of loading conditions, which might contribute to the reduction of the overall stress levels of the re-entrant wall MN through bending deformation. For the 2D diamond structure, the normalized principal stress level is always larger than unity, which suggests that the finite boundary effects result in the weakening of the structural strength. The deformation characteristics of all the walls of the ideal 2D diamond structure is identical. However, with finite boundary effect the deformations of individual walls become inconsistent, which might result in the increase of principal stress levels for some walls. 

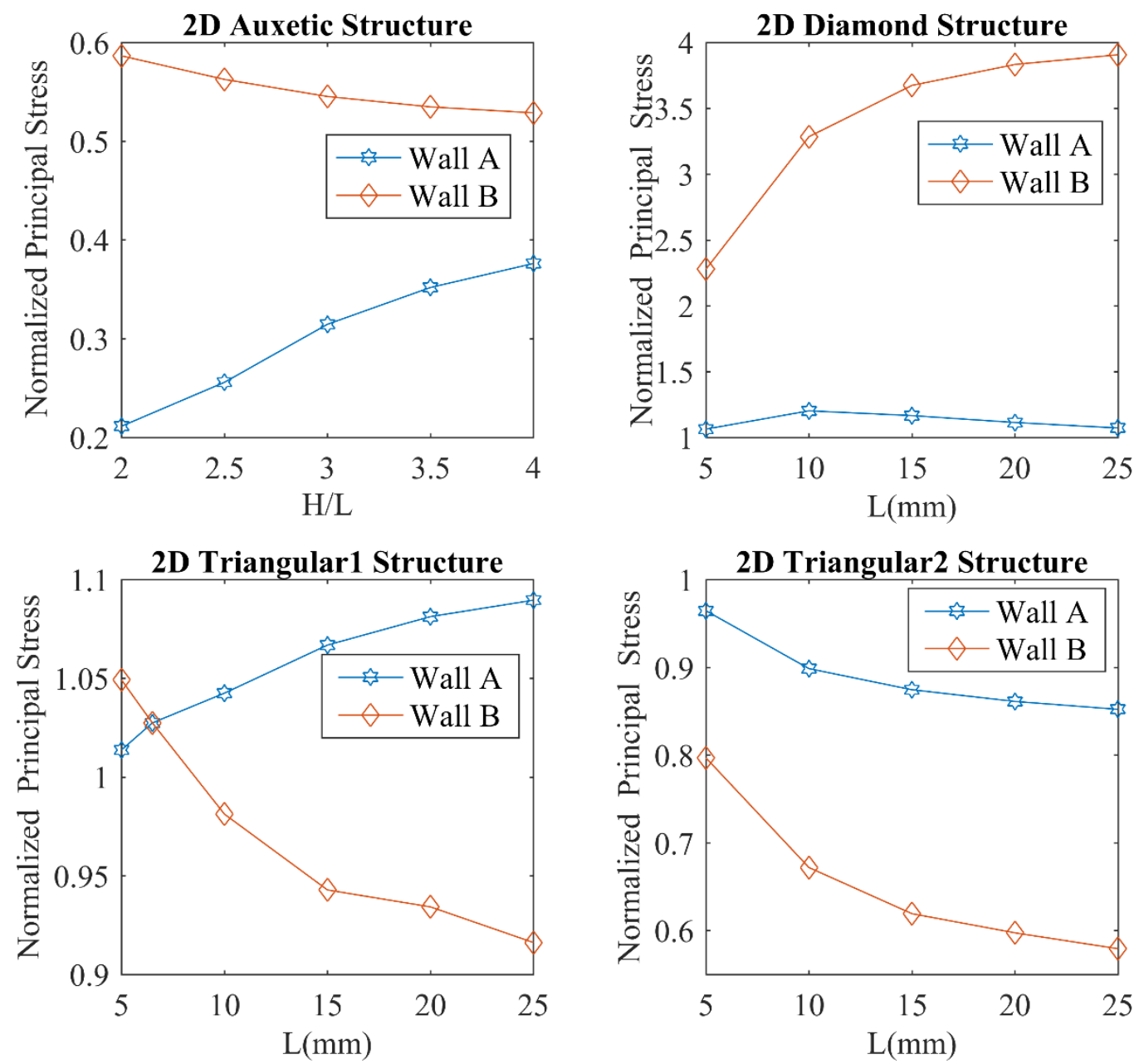

Fig. 3. 11 The comparison of the normalized principal stresses of wall A and wall B for four structures

The failure pattern mode change with the 2D triangular1 structure was further analyzed in order to identify the driving factor. The force and stress components of wall A and wall B of 2D triangular1 is shown in Fig. 3.12. From Fig. 3.12, the bending moments and shear forces bot exhibit decreasing trend when the wall length becomes longer, while the axial force remains consistent, as would be deducted from the classic theory for a typical stretching-dominated structure. In this study, the bending moments $(M)$ and shear force $\left(F_{y}\right)$ in wall $\mathrm{B}$ are both significantly higher than that in wall $\mathrm{A}$, while the axial force 
$(F x)$ in wall B was much smaller than that in wall A. Based on the parameters setting of 2D triangular1 structures in this study, the normal stress $\sigma$ and shear stress $\tau$ can be expressed as

$$
\begin{gathered}
\sigma=0.6 M+0.1 F_{x} \\
\tau=0.15 F_{y}
\end{gathered}
$$

From Equation (3.3), it can be deducted that higher bending moment in wall $\mathrm{B}$ could predominantly contribute to higher normal stress even though it is subjected to lower axial force. For designs with shorter wall length, the bending of wall $\mathrm{A}$ is smaller than that of wall B, possibly due to the boundary constraint. However, as the wall length increases (and correspondingly bending moment reduces), the bending moment variation in wall A is also significantly smaller than that of wall B. As a result, for designs with longer wall length, wall A would exhibit higher overall normal stress levels compared to wall B. Furthermore, from Equation (3.4) it could also be seen that the shear stress (shown in Fig. 3.12(e)) resulted from the shear force (shown in Fig. 3.12(b)) contributes significantly less to principal stress compared to the normal stress. Lastly, such discussions also clearly suggest that the bending also plays a significant role in the failure characteristic of the stretching-dominated structures. 

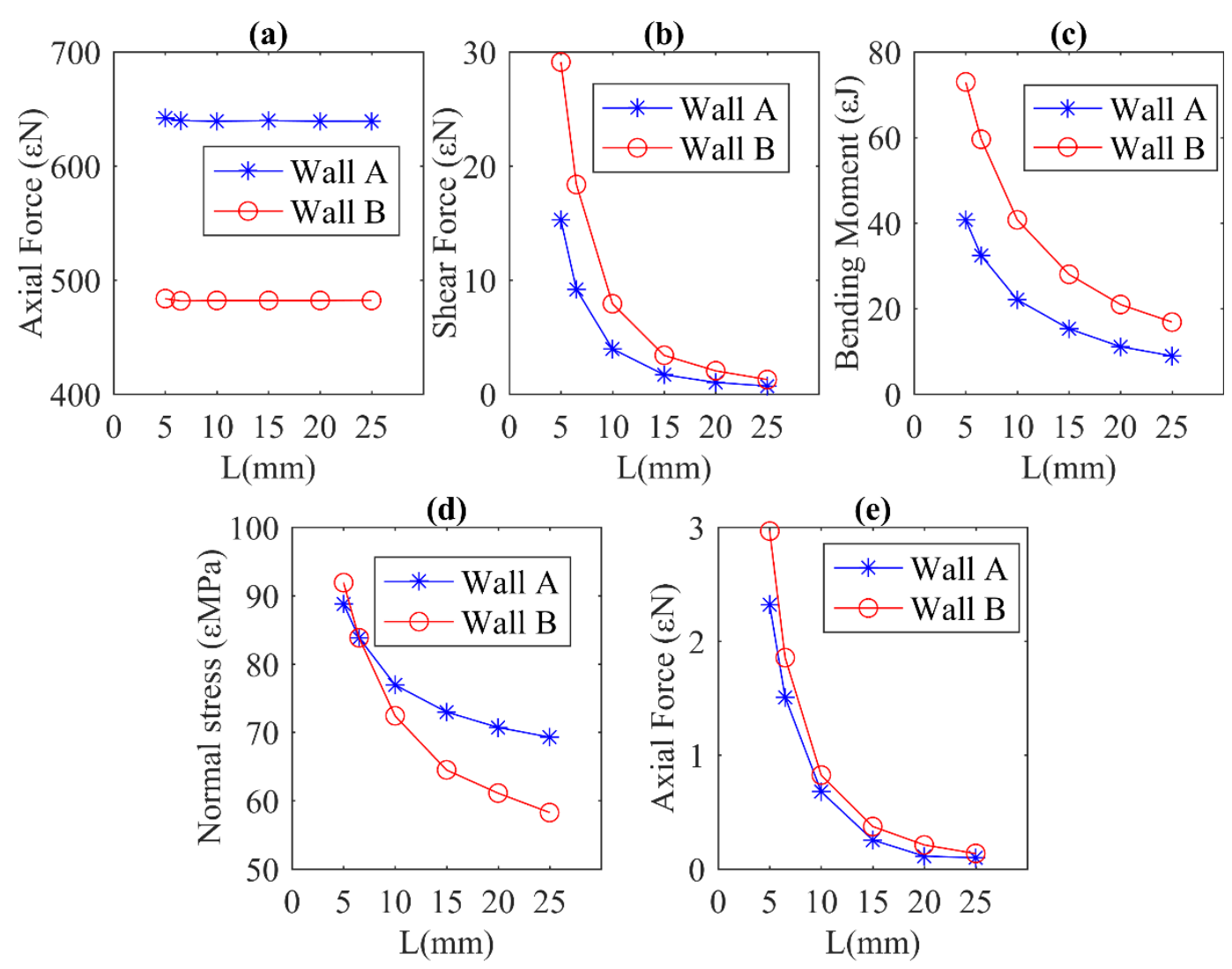

Fig. 3. 12 Forces and stresses analysis of the of wall A and wall B of 2D triangular1 structure: (a)Axial force analysis;(b) Shear force analysis;(c) Bending moment analysis; (d) Normal stress analysis; (e) Shear stress analysis

\subsection{Size-topology effect of 3D cellular structures}

For the 3D cellular structures, the size-topology effect on their mechanical properties and failure response is also investigated in this section. Since the focus of this section is on the size-topology effect, only unit cell numbers and unit cell topology will be discussed in this section for 3D cellular structures. 


\subsubsection{Geometry design and analysis}

In this study, BCC, Octahedral, Octet-truss and Auxetic structures are used as the basic unit cells shown in Fig. 3.13. All four cellular designs have been designed and realized via AM and widely studied for mechanical properties. These cellular designs were selected to investigate the potential relationship between size effects and tensile fracture properties. Among these designs, the auxetic structure exhibits negative Poisson's ratios, the octet-truss structure exhibits high modulus and stretch-dominated deformation, while the BCC cellular and octahedral structures both exhibits bending-dominated deformation. In Fig. 3.13, the size of the red cube was set as $12 \mathrm{~mm} \times 12 \mathrm{~mm} \times 12 \mathrm{~mm}$, which defines the bounding volume of the cellular unit cells. The diameter of struts was set as $1 \mathrm{~mm}$ for all the structures. For the BCC structure (relative density of 4.44\%) in Fig. 3.13(a), the strut length was set as $10 \mathrm{~mm}$. For the octahedral structure (relative density of $8.45 \%$ ) in Fig. 3.13(b), the oblique strut length was set as $10 \mathrm{~mm}$ and the horizontal strut length was set as $14 \mathrm{~mm}$. For the octet-truss structure (relative density of 15.62\%) in Fig. 3.13(c), the strut length was set as $8 \mathrm{~mm}$. For the auxetic structure (relative density of 12.78\%) in Fig. 3.13(d), the opening angle was set as 60degree, the re-entrant strut length was set as $6.7 \mathrm{~mm}$ and the vertical strut length was set as $9.1 \mathrm{~mm}$. For all of these structures, the unit cell numbers vary from $2 \times 2 \times 2$ to $8 \times 8 \times 8$. Ti- $6 \mathrm{Al}-4 \mathrm{~V}$ was arbitrarily selected as the material in the analytical calculation with the Young's modulus of $114 \mathrm{GPa}$, the shear modulus of 43GPa, and the yield strength of 1050MPa. As the study was not intended to investigate material effects, no further treatment was implemented to the material property setting, and a simple perfectly elastic material model was assumed. The tensile process of the four types cellular structures were numerically simulated through the proposed model in Section 2.2.2. The 
effect of the unit cell numbers on the tensile failure patterns, the tensile strength, the modulus and energy absorption were analyzed.

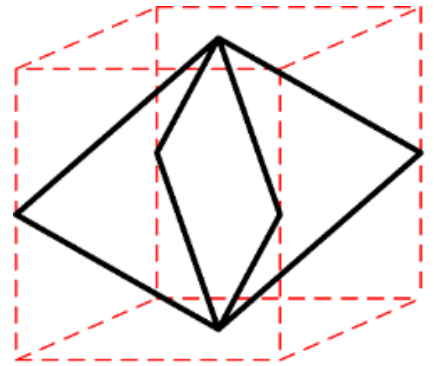

(a)

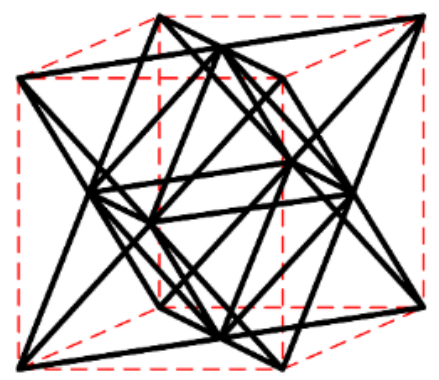

(c)

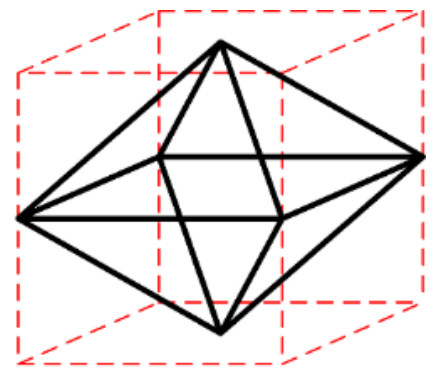

(b)

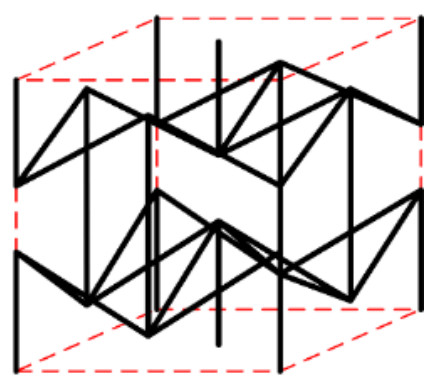

(d)

Fig. 3.13 (a) BCC; (b) Octahedral; (c) Octet-truss; (d) Auxetic

\subsubsection{Tensile failure responses of four structures}

To investigate the effect of the unit cell numbers of the structure on the tensile response, the strain-stress responses of all the four structures were analyzed, shown in Fig. 3.14. In Fig. 3.14, the $\mathrm{x}$-axis and $\mathrm{y}$-axis of each curve indicate the strain and stress respectively. For all the four structures, the strain-stress curves of the strut failure exhibit the perfect elastic-brittle failure characteristics typical to the brittle materials with maximum stress failure mode that was adopted in this study. For the BCC, octahedral and octet-truss structures, the strain-stress curves exhibit some obvious saw tooth-like patterns, with critical stress levels decrease. For the auxetic structure, the strain-stress curves just exhibit a single stress peak, which indicate a catastrophic failure. 
(a)

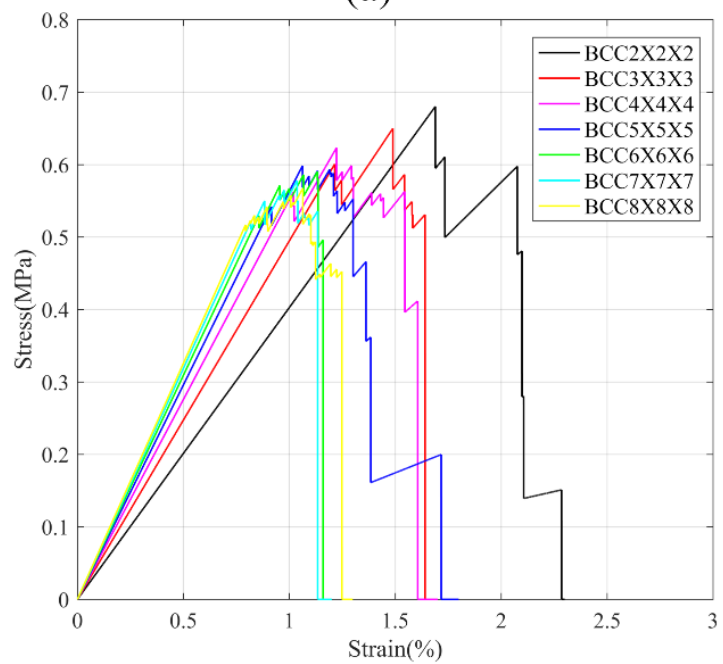

(c)

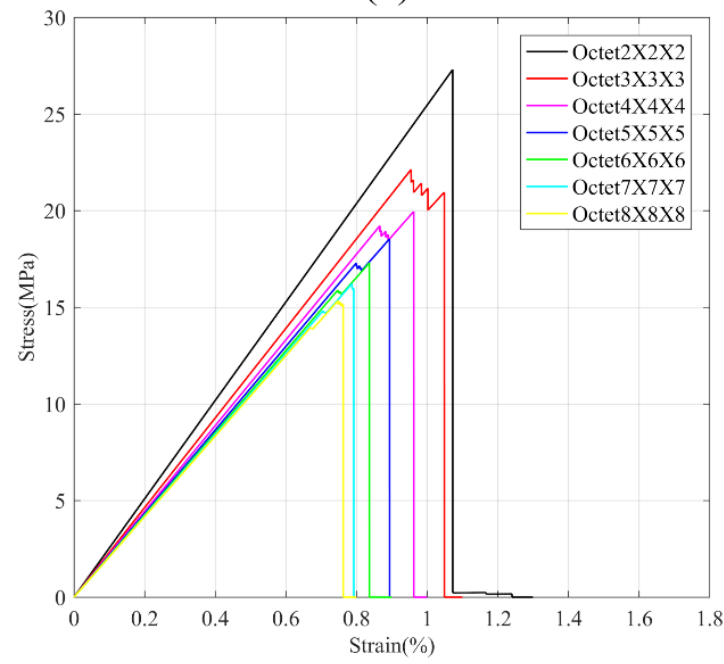

(b)

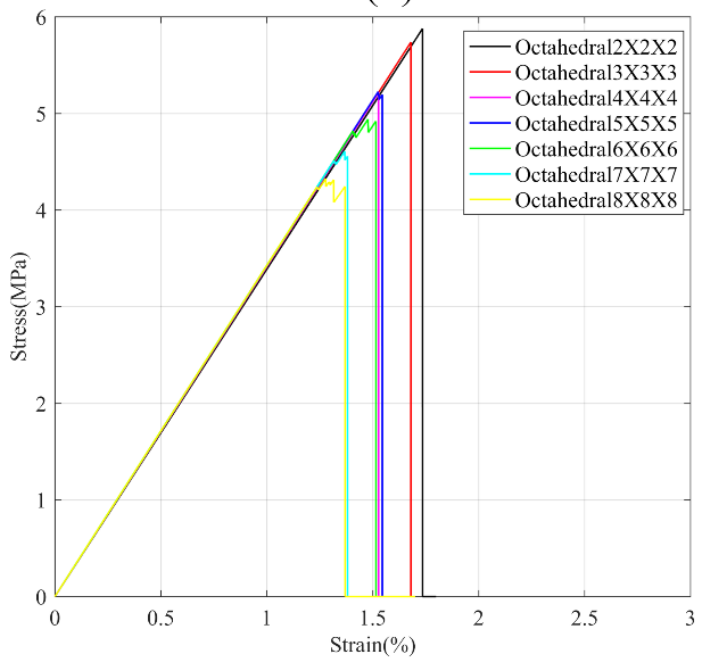

(d)

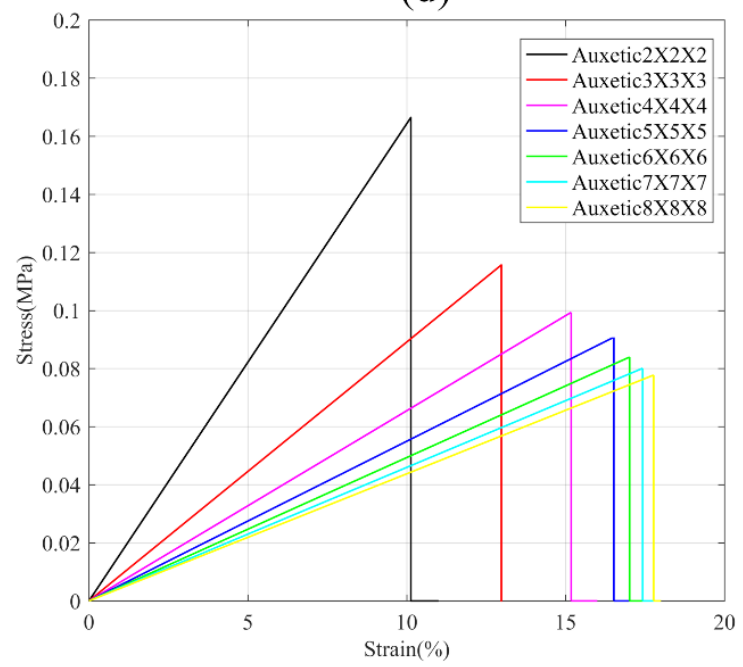

Fig. 3. 14 The strain-stress curves of four structures with different unit cell numbers: (a)

BCC; (b) Octahedral; (c) Octet-truss; (d) Auxetic

It is worth noting that even though the $\mathrm{BCC}$, octahedral and octet-truss structures exhibit the saw tooth-like strain-stress curves, the distribution and the value of these stress peaks are significantly different. For the BCC structures shown in Fig. 3.14(a), the stress peaks distribute more uniformly from the first fracture unit the total failure of the structure. For the octahedral shown in Fig. 3.14(b) and octet-truss shown in Fig. 3.14(c), the distribution of their stress peaks is more concentrated. And stress values of these peaks are 
closer to the first peak. From the corresponding strains of these peaks, we can also see that the fracture process of these three structures is a progressive fracture evolution which is similar to the crack propagation. Among these three structures, the BCC structure shows a relatively stable and slow crack propagation compared with the octahedral and octet-truss structures. For the auxetic structures shown in Fig. 3.14(d), the fracture pattern is more catastrophic, the whole structure fails right after the fracture initiates.

3.3.3 Tensile strength, Young's modulus and energy absorption analysis of four structures

The effect of the unit cell numbers on the normalized tensile strength (the tensile strength divided by the relative density) was shown in Fig. 3.15(a). From Fig. 3.15(a), it can be seen that the normalized tensile strength of all the four structures decreased when the unit cell numbers increased. The octahedral and octet-truss structures exhibited much higher normalized tensile strengths than that of the auxetic and BCC structures. Besides, both the BCC and the auxetic structures exhibit relatively consistent strength levels with varying unit cell numbers, in comparison with the other two types of structures. Such observation also contradicts with the previous suggestion of the size effects with these structures, in which the size effects appear to converge when the vertical (i.e. along the loading direction) numbers of unit cells are identical to the lateral number of unit cells. While additional investigation of this subject is required, it was speculated that the discrepancies could be at least partly attributed to the different methods utilized for the calculations and the different geometrical parameter settings for the cellular designs. 

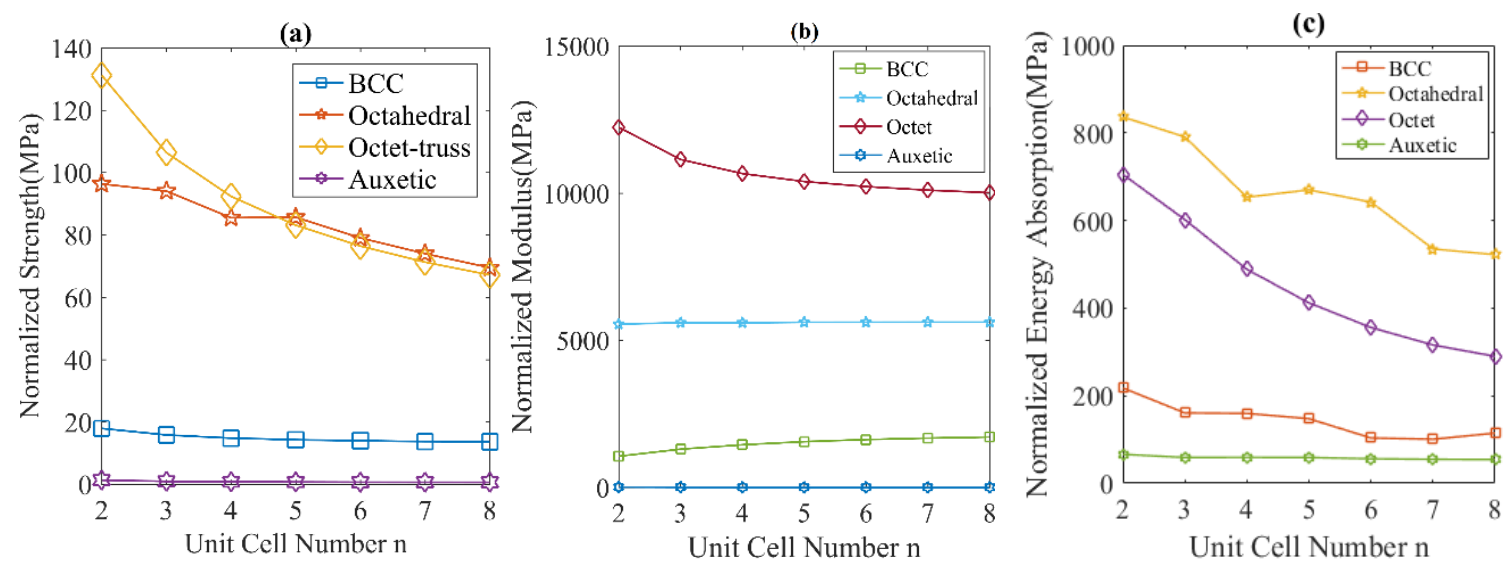

Fig. 3. 15 Size effects on the normalized tensile strength (a), normalized Young's modulus (b) and normalized energy absorption (c) of four structures

The effect of the unit cell numbers on the normalized Young's modulus (the Young's modulus divided by the relative density) was shown in Fig. 3.15(b). The octettruss structures exhibit the highest modulus at all unit cell number range, while the auxetic structures exhibit the lowest. For the octet-truss structures, he normalized Young's modulus also exhibit most significant decreasing trend when the unit cell numbers increase. On the other hand, for the other types of structures, the trends appear much less significant.

The effect of the unit cell numbers on the normalized energy absorption (the energy absorption divided by the relative density) was shown in Fig. 3.15(c). For perfectly elastic materials, the energy absorption is determined by both the maximum strength and the elastic modulus. From the results, the normalized energy absorptions of all the structures decreased when the unit cell number increased, which agree with the trends observed from the normalized elastic modulus and strength. For the auxetic structures, it is expected that the size effect is minimized, and therefore the energy absorption characteristics should also exhibit minimum size effects. On the other hand, with the other structures, the size effects are introduced either through reduced elastic modulus or reduced maximum strength. 


\subsubsection{Tensile failure pattern of four structures}

Using the proposed fracture model, the predicted tensile failure patterns of four structures are shown in Fig. 3.16 to Fig. 3.19 respectively. Fig. 3.16 shows the tensile failure patterns of the BCC structures. From Fig. 3.16, the BCC structures exhibit a diagonal or $\mathrm{V}$ shape fracture patterns when the unit cell numbers are smaller than $8 \mathrm{x} 8 \mathrm{x} 8$. When the unit cell numbers increase beyond 8 , the fracture patterns exhibit another consistent fracture pattern located in the middle. Combining the strain-stress curves shown in Fig. 3.14(a), it is seen that the structures undergo more fracture progression steps (more stress peaks in the strain-stress curves indicate more fracture steps) before the total failure of the structure. In addition, for the BCC structures with larger unit cell numbers, prior to the occurrence of the primary fracture path located in the middle layer, some of the struts located in the corner would crack first. 


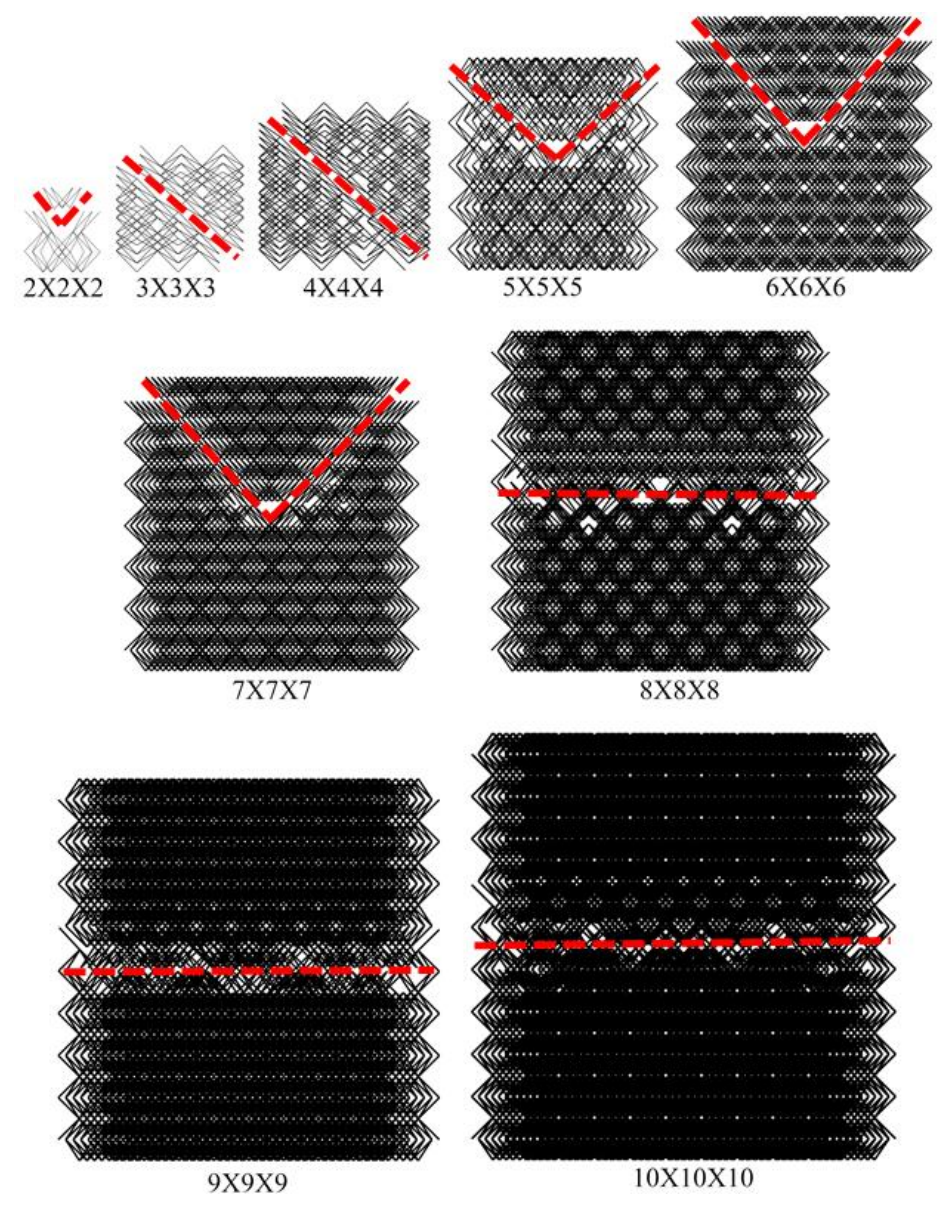

Fig. 3. 16 Fracture patterns of BCC structures with different unit cell numbers

Fig. 3.17 shows the tensile failure patterns of the octahedral structures. When the unit cell numbers are smaller than 6x6x6, the fracture path was located in the middle layer of the structures. When the unit cell numbers are larger than $5 \times 5 \times 5$, the fracture exhibited a more tortuous pathway that transitions from the corner to the middle. And also from Fig. $3.14 \mathrm{~b}$, for the structures with smaller unit cell numbers, the fracture tend to be more catastrophic. When the fracture starts, the structure fails immediately. In contrast, for the structures with larger unit cell numbers, the fracture tends to have more steps. The structures can retain most of the overall strength after some early crack steps. This might 
provide a potentially useful design guideline for choosing the unit cell numbers for the design of fracture toughness of the BCC structures.

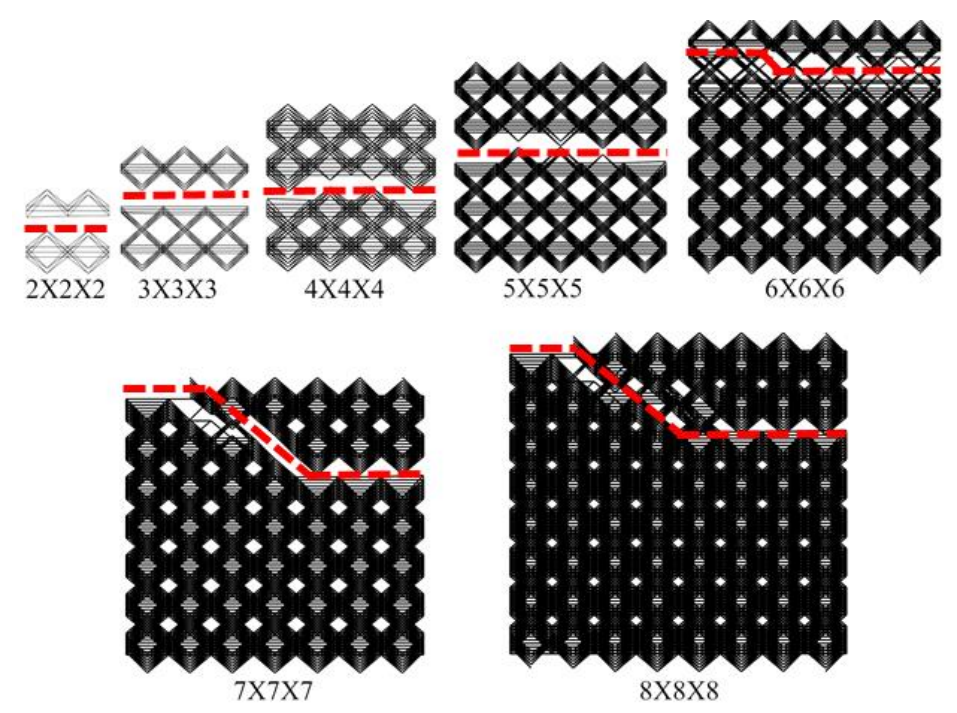

Fig. 3. 17 Fracture patterns of octahedral structures with different unit cell numbers

Fig. 3.18 shows the tensile failure patterns of the octet-truss structures. The fracture patterns are relatively consistent with different unit cell numbers. The fracture initiates at the corner of the structures, and then propagates towards the middle region of the structures. From Fig. 3.14@, it is seen that the octet-truss structures have a similar crack propagation process with the octahedral structures. The structures with larger unit cell numbers tend to exhibit more stable crack propagation process before the total failure of the structures. 


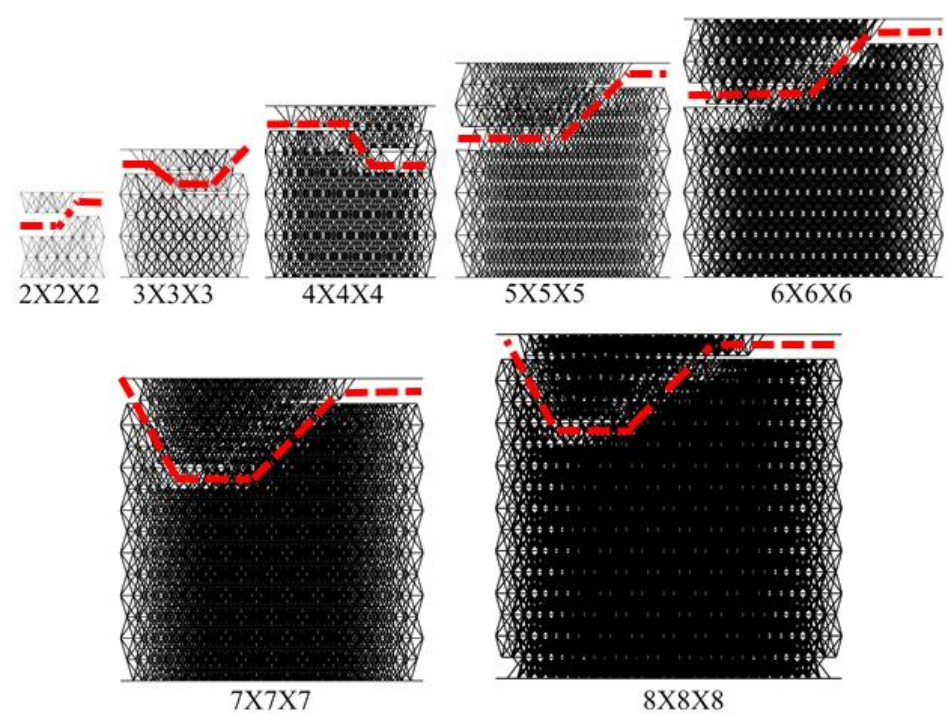

Fig. 3. 18 Fracture patterns of octet-truss structures with different unit cell numbers

Fig. 3.19 shows the tensile failure patterns of the auxetic structures. The fracture patterns are highly consistent across different unit cell numbers. For the structures with smaller unit cell numbers, the fractures locate at the boundary layers. For the structures with larger unit cell numbers, the fractures occur at the second layers. From the Fig. 3.14(d), it is seen that the strain-stress curves have only one stress peaks, which indicates that the structure will fail immediately and lose all the loading capacity once the fracture starts. Such distinct "layerwise" fracture pattern was also experimentally observed in previous literature, although it is also speculated that such behavior might be specific to certain geometry design parameter ranges. 


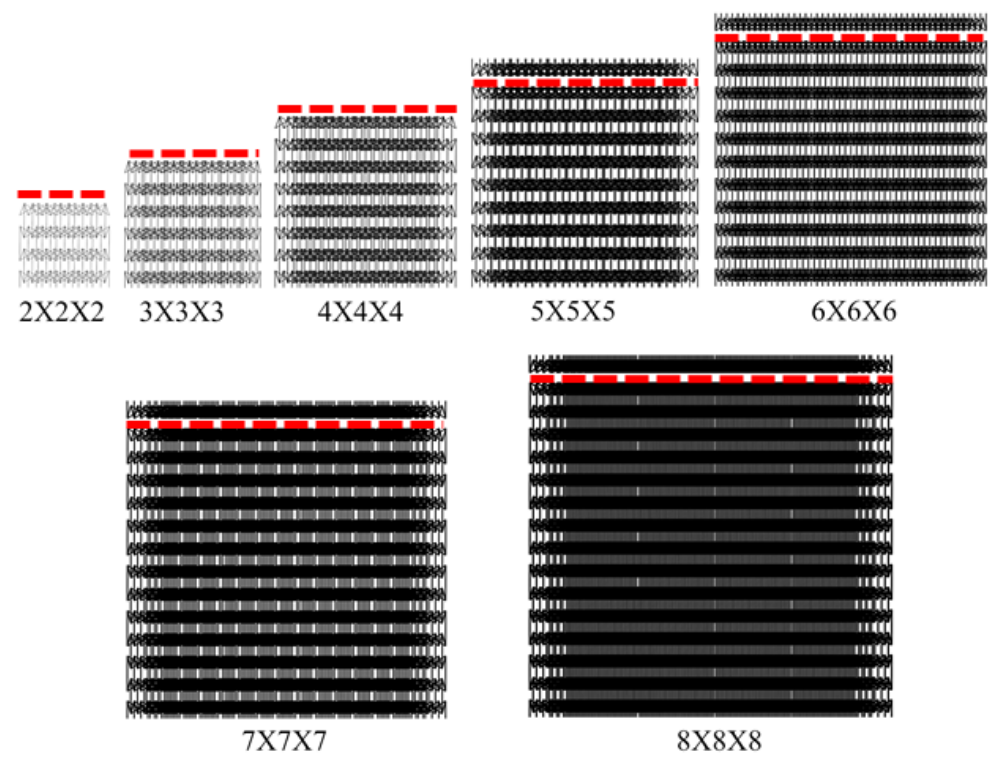

Fig. 3. 19 Fracture patterns of auxetic structures with different unit cell numbers

\subsection{Conclusions}

In this section, the mechanical property and failure behavior of both $2 \mathrm{D}$ and $3 \mathrm{D}$ cellular structures have been analyzed based on the proposed model. The failure behavior of each cellular structure is characterized by 2 steps: (i) initial failure of the perfect structure, and (ii) the crack propagation after the initial failure. The findings presented in this section demonstrate the following conclusions:

1. The failure pattern analysis showed that the cellular design type significantly affects the fracture pattern. Generally, for the 2D cellular structures, the 2D bendingdominated structures with lower nodal connectivity exhibited relatively stable crack propagation pattern, while 2D stretching-dominated structures with higher nodal connectivity appear to exhibit rather catastrophic fracture failure. For 3D cellular structures, the fracture process of the $\mathrm{BCC}$ structure tends to be a progressive fracture evolution, while for the octahedral and octet-truss structures, the fracture process experiences less fracture 
stages compared with the BCC structures. The auxetic structure appears to exhibit rather catastrophic fracture failure.

2. For the 2D cellular structures, the energy absorption analysis showed that under the same relative densities, the energy absorption of two 2D stretching-dominated structures were significantly higher than that of two 2D bending-dominated structures. And generally, the energy absorption for these four structures follow the changing trend with the relative density. However, it was found that the normalized energy absorption decreased with an increased relative density, which provides a design insight about the need to balance the relative density, energy absorption, and normalized energy absorption.

3. The size effect analysis showed that the failure behavior (including the Young's modulus, tensile strength, energy absorption and failure pattern) tends to converge to consistent values when the unit cell numbers increase sufficiently, even though different structures may have different characteristic stabilization unit cell numbers. 


\section{CHAPTER IV}

\section{MODELING AND ANALYSIS OF MATERIAL ANISOTROPY-TOPOLOGY \\ EFFECTS OF 3D CELLULAR STRUCTURES FABRICATED BY POWDER BED FUSION ADDITIVE MANUFACTURING}

\subsection{Introduction}

AM process provides great potentials for the fabrication of complex cellular structures. Large amount of works has been dedicated to the characterization of AM cellular structures. Among the factors that influences the properties and qualities of the AM cellular structures, material anisotropy is a significant one, which significantly related with the strut angles. Even though literatures have demonstrated the important effect of the material anisotropy on the mechanical properties of cellular structure, one of the outstanding issues with the design of AM cellular structures is the lack of an efficient tool to account for material anisotropy and extrinsic defects during the evaluation of the overall properties of the structures. FEA provides a brutal force mean to obtain mechanical property estimation results for individual cases, which remains an attractive alternative due to its high fidelity. However, FEA tends to be computationally inhibitive, especially when the cellular topology becomes more complex and the cellular structure pattern size increases. In addition, from the design's perspective, the case-by-base based FEA is often not most efficient in generating optimized design insights. A more comprehensive analytical-based model has the potential of both facilitating the discovery of design characterizations and the optimization of designs. Particularly, as the material anisotropy 
effect is directly related to the topological design of the cellular unit cells, an integrated model that could investigate the coupled material anisotropy-cellular topology effects is desirable. Although such model are sometimes difficult to solve analytically, they allow for both explicit analysis of the impacts of individual design variables (and their combinations) and relatively straightforward numerical solutions which, despite being not as accurate as FEA, conveniently enables rapid design screening and preliminary evaluation.

In this section, the focus is to establish an analytical model based on the proposed model in Section 2.2.2 for the analysis of general periodic cellular structures with anisotropic material properties fabricated via PBF. The material anisotropy information will be established experimentally using single struts with different build orientations $\left(0^{\circ}\right.$, $15^{\circ}, 30^{\circ}, 45^{\circ}, 60^{\circ}, 75^{\circ}$ and $90^{\circ}$ ) and then be incorporated into the analytical model. In order to ensure adequate fidelity of the model, a non-homogenization direct stiffness matrixbased modeling approach was adopted, which aims to capture the significant effect of the boundary constraints imposed from the finite-size cellular structures that are common for AM cellular structure designs. Cellular structures fabricated via EB-PBF were utilized for experimental verification, although the model was expected to be applicable for other AM systems and materials.

\subsection{Cellular structure designs}

Fig. 4.1 shows three different cellular structures investigated in this study, including the re-entrant auxetic structure, the BCC structure, and the octahedral structure. These designs were selected due to their significantly different topological characteristics. The re-entrant auxetic structure exhibits negative Poisson's ratio along all three principal 
directions. The $\mathrm{BCC}$ structure is a typical bending-dominated structures, whereas the octahedral structure is a stretch-dominated structure that is topologically similar to the BCC structure. Without losing generality, it was assumed in the model that the cross-sectional shape of the struts in all three cellular structure is square. For the auxetic structure (Fig. 4.1(a)), there are 4 primary design parameters for the unit cell structure: the length of the vertical struts $H$, the length of the re-entrant struts $L$, the re-entrant angle $\theta$, and the thickness of the strut cross section $t$. For both the BCC structure (Fig.4.1(b)) and octahedral structure (Fig. 4.1(c)), there are three design parameters for the unit cell structure: the length of all the inclined struts $L$, the strut angle $\theta$, and the thickness of the strut cross section $t$.

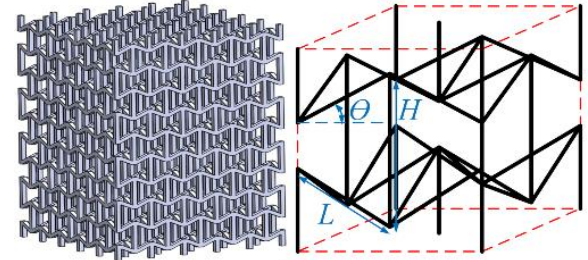

(a)

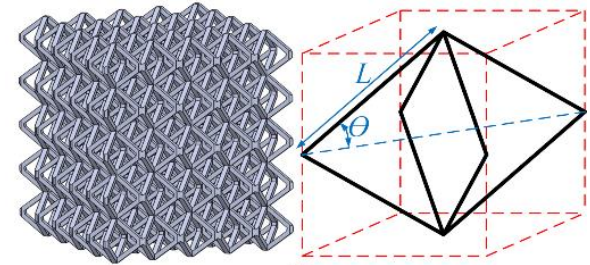

(b)

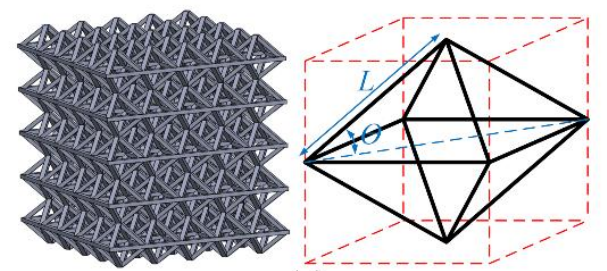

(c)

Fig. 4. 1 Cellular structures and unit cells: (a) auxetic; (b) BCC; (c) octahedral

The stiffness matrix method in Section 2.2.2 was used to model these cellular structures. In the proposed model, since each individual strut is separately modeled using its unique element stiffness matrix shown Equation (2.11)-(2.14), the material anisotropy (different material modulus $E$ for different struts) can be easily incorporated into each element stiffness matrix. Through assembling all different stiffness matrices for all struts 
in the cellular structures into a global stiffness matrix for the entire structure, the material modulus anisotropy can be modeled in the cellular structures. Then combining the maximum principal stress criteria (Section 2.3.2), the material strength anisotropy is further added to the model.

4.3 Evaluation of material anisotropy of the strut with different angles

In the experimental verification of the material anisotropy effects of the cellular structures, the EB-PBF process was arbitrarily selected, which is currently a comparatively advantageous PBF process for the fabrication of cellular structures. To establish the material anisotropy baseline, struts with different orientations $\left(0^{\circ}, 15^{\circ}, 30^{\circ}, 45^{\circ}, 60^{\circ}, 75^{\circ}\right.$ and $90^{\circ}$ ) in respect to the building direction ( $z$-direction) were designed and manufactured by an Arcam EB-PBF S400 system using Ti6Al4V powder (AP\&C, 44-105 $\mu m$, plasma rotating electrode process). As the study mainly focused on the design aspects of the material anisotropy-topology effects, no special attempt was made to characterize the powder feedstock characteristics, or to optimize the system or process parameters. The default canned process parameters for the cellular structures (Ti6Al4V-Network) was used for the fabrication. The layer thickness was set to $50 \mu \mathrm{m}$. During the process, the powder bed was first preheated by a slightly defocused beam with beam current gradually ramped from 0 up to $35 m A$ over a span of 17 seconds, per the default setting. Preheating lightly sinters the powder bed in order to produce a more stable layer condition upon subsequent melting. Preheating also introduces an elevated powder bed temperature that reduces thermally induced stresses in the fabricated parts. Following preheating, the struts were melted using a beam current setting of $4.5 \mathrm{~mA}$. The exact beam current is geometry specific and is determined by the control algorithm's proprietary functions. 
The designs of the tensile samples are shown in Fig. 4.2(a). All struts have $1 \times 1 \mathrm{~mm}^{2}$ square cross section, consistent to the strut dimensions used for the subsequent design of cellular structures. The fabricated struts are shown in Fig. 4.2(b). Fig. 4.2(c) illustrates the orientation effects of the cellular struts. In comparison, the $90^{\circ}$ struts exhibit relatively homogenous surface textures. As the strut orientation decreases, the surface textures become rougher and less regular, especially with the down-facing side. It is well known that the staircase effect is an important factor for the reduced quality of low-orientation support-free features. In addition, the high ambient powder bed temperature $\left(\sim 600-800^{\circ} \mathrm{C}\right.$ throughout the process) of the EB-PBF also results in increased powder sintering and sticking, contributing to the further reduction of the surface qualities of the struts. The anisotropic mechanical responses of the struts are affected by a combination of all these factors including build orientation, microstructures, thermal history, porosity and surface roughness. Instead of modeling these factors for the material anisotropy, the experimentbased strut mechanical properties are directly used. 
(a)

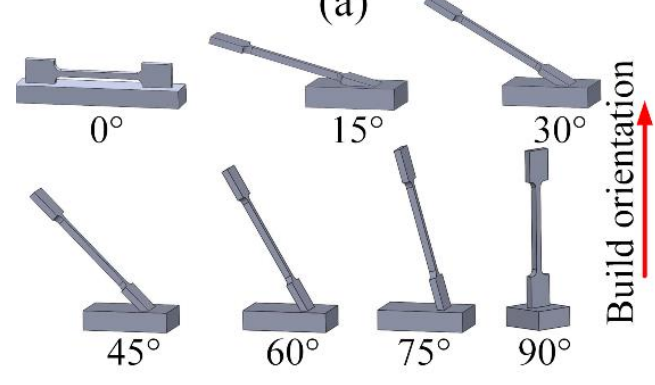

(b)

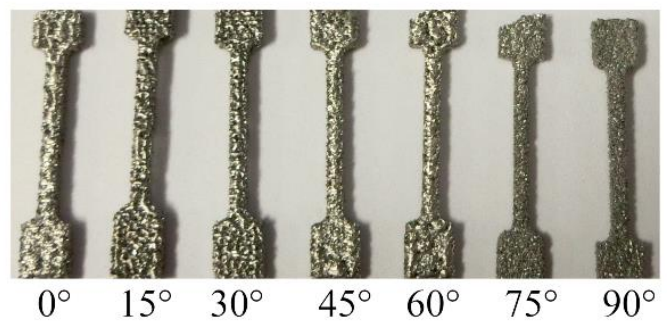

(c)

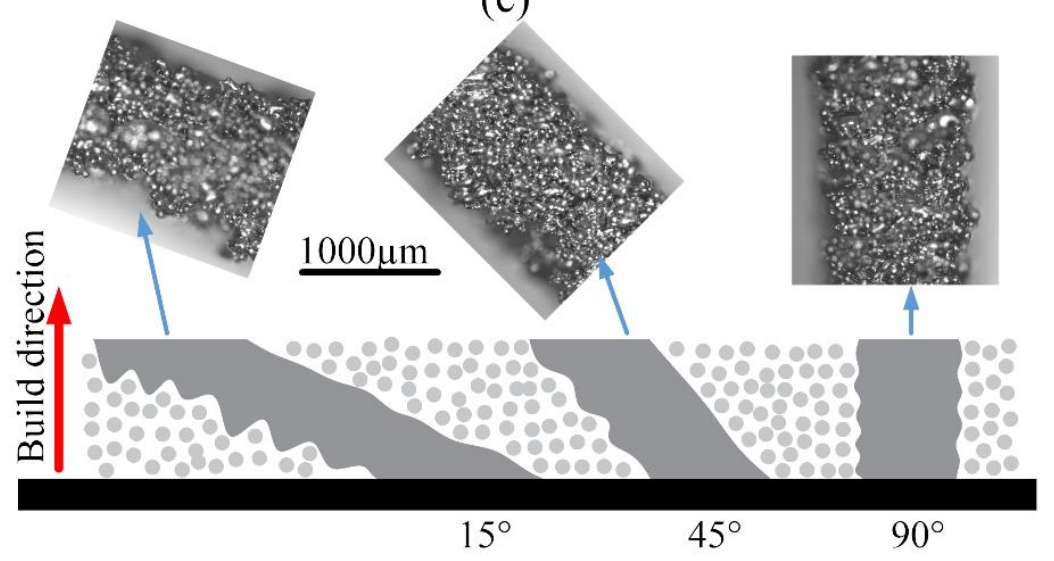

Fig. 4. 2 (a) Design and (b)fabrication of the single strut with different angles, and (c) sample surface of struts with different angles produced by EB-PBF process

5 replicas of each strut orientation were fabricated. All the sample were fabricated without using supporting structures. The yield strength and Young's modulus of each strut were obtained through tensile testing, which were conducted at the Instron 5569A tensile testing machine $(5 \mathrm{kN}$ load cell) with a constant strain rate of $0.3 \mathrm{~mm} / \mathrm{min}$. Fig.5a shows the representative strain-stress curves of the single struts of different orientation angles. From the curves, it can be seen that most struts exhibit relatively small plastic yield prior to failure, which validates the applicability of the maximum stress failure criteria adopted in the model. Fig. 4.3(b) and Table 4.1 show the anisotropic effects. The isotropic mechanical property of EB-PBF solid Ti6Al4V (with modulus of $114 \mathrm{GPa}$ and ultimate strength of $1.05 G P a$ ) were utilized to provide a reference baseline for the evaluation of the effect of 
the material anisotropy on the mechanical properties of cellular structures, which are presented by the relative mechanical properties (absolute mechanical property divided by corresponding properties of the reference solid material) in both Fig. $4.3 \mathrm{~b}$ and Table 4.1 . As the strut angle increases from $0^{\circ}$ to $90^{\circ}$, the yield strength and Young's modulus increase from $364 \mathrm{MPa}$ to $877 \mathrm{MPa}$ and from $25 \mathrm{GPa}$ to $53 \mathrm{GPa}$, respectively. The elastic modulus of the struts appears to exhibit less consistent anisotropic trend. As process quality investigation is beyond the scope of this study, the results were not further discussed. Subsequently, the mechanical property values were defined in the analytical modeling for individual struts based on their respective orientation angles from Table 4.1.
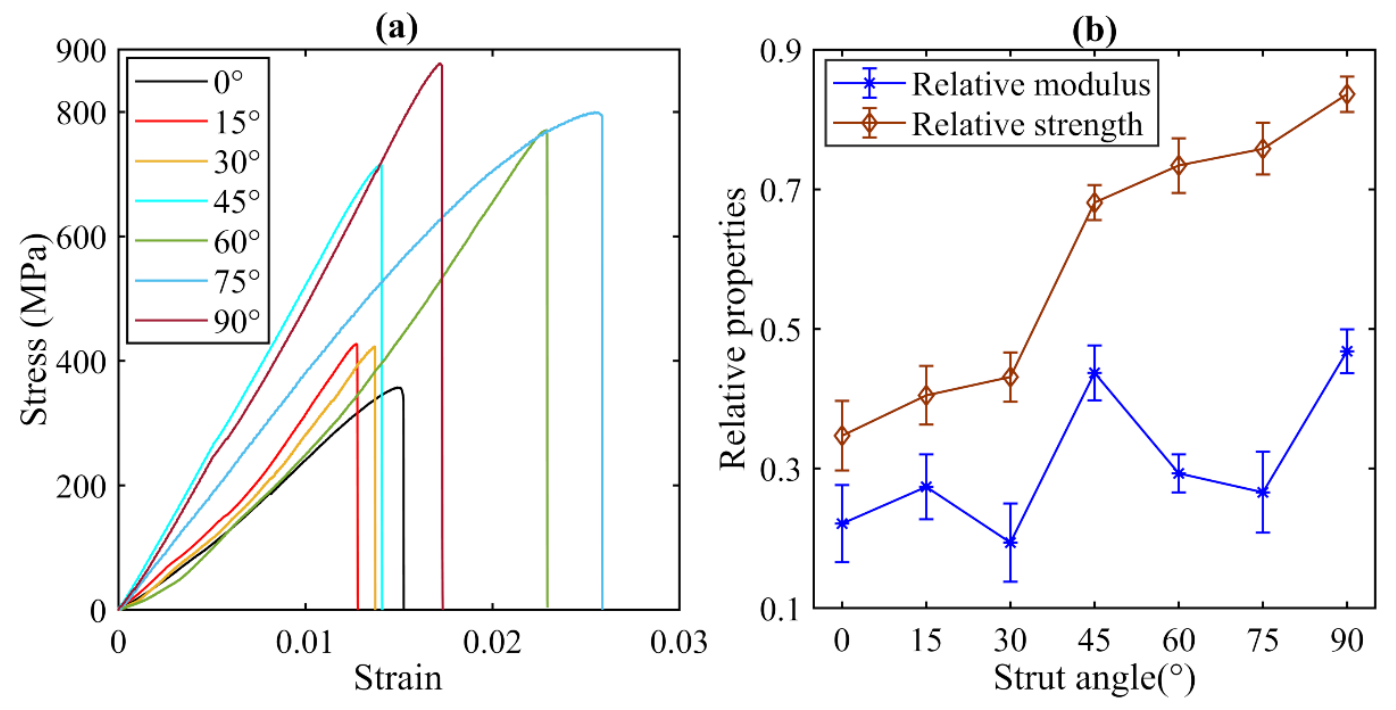

Fig. 4.3 (a) Strain-stress curve of the single struts with different angles; (b) The relationship between the strut angle and relative strength and modulus (normalized by the solid strength and modulus)

Table 4. 1 Experimental mechanical properties of the single struts with different orientations

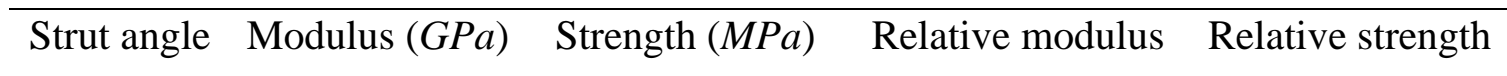




\begin{tabular}{ccccc}
\hline $0^{\circ}$ & $25.2 \pm 6.3$ & $364.8 \pm 52.2$ & 0.221 & 0.347 \\
$15^{\circ}$ & $31.2 \pm 5.3$ & $424.8 \pm 44.2$ & 0.274 & 0.405 \\
$30^{\circ}$ & $22.1 \pm 6.4$ & $452.3 \pm 37.3$ & 0.194 & 0.431 \\
$45^{\circ}$ & $49.8 \pm 4.5$ & $715.1 \pm 26.4$ & 0.437 & 0.681 \\
$60^{\circ}$ & $33.4 \pm 3.1$ & $770.7 \pm 41.1$ & 0.293 & 0.734 \\
$75^{\circ}$ & $30.3 \pm 6.6$ & $795.0 \pm 38.9$ & 0.266 & 0.758 \\
$90^{\circ}$ & $53.4 \pm 3.6$ & $877.8 \pm 26.5$ & 0.468 & 0.836 \\
\hline
\end{tabular}

4.4 Effect of the material anisotropy-topology on structural mechanical properties

4.4.1 Effect of the material anisotropy on structural mechanical properties

The effect of material anisotropy on the overall properties of the cellular structures were calculated for the three cellular unit cell designs of various pattern sizes. In the modeling-based study, for each type of cellular unit cells, structures of 5 different strut angle $\theta$ levels $\left(15^{\circ}, 30^{\circ}, 45^{\circ}, 60^{\circ}, 75^{\circ}\right)$ were considered. The only exception was with auxetic design, for which a strut angle $75^{\circ}$ resulted in a physically infeasible geometry and was therefore excluded from the design. The designed models are illustrated in Fig. 4.4. For each types of unit cells, all the other geometry design parameters were kept constant. The numbers of unit cells, or pattern sizes, of the cellular patterns were kept constant as $5 \times 5 \times 5$ for the initial study, in order to focus on the comparison of material anisotropy effects on cellular structures of different unit cell topologies. All the struts were designed to have square cross sections with thickness of $1 \mathrm{~mm}$. For the auxetic structures, the lengths of the vertical struts $H$ and re-entrant struts $L$ were set at $9 \mathrm{~mm}$ and $4.5 \mathrm{~mm}$ respectively. For the BCC and octahedral structures, the length of all the inclined struts $L$ was set at $8 \mathrm{~mm}$. For each design, the mechanical properties of the structure based on both anisotropic and isotropic material properties were calculated from the analytical model. 


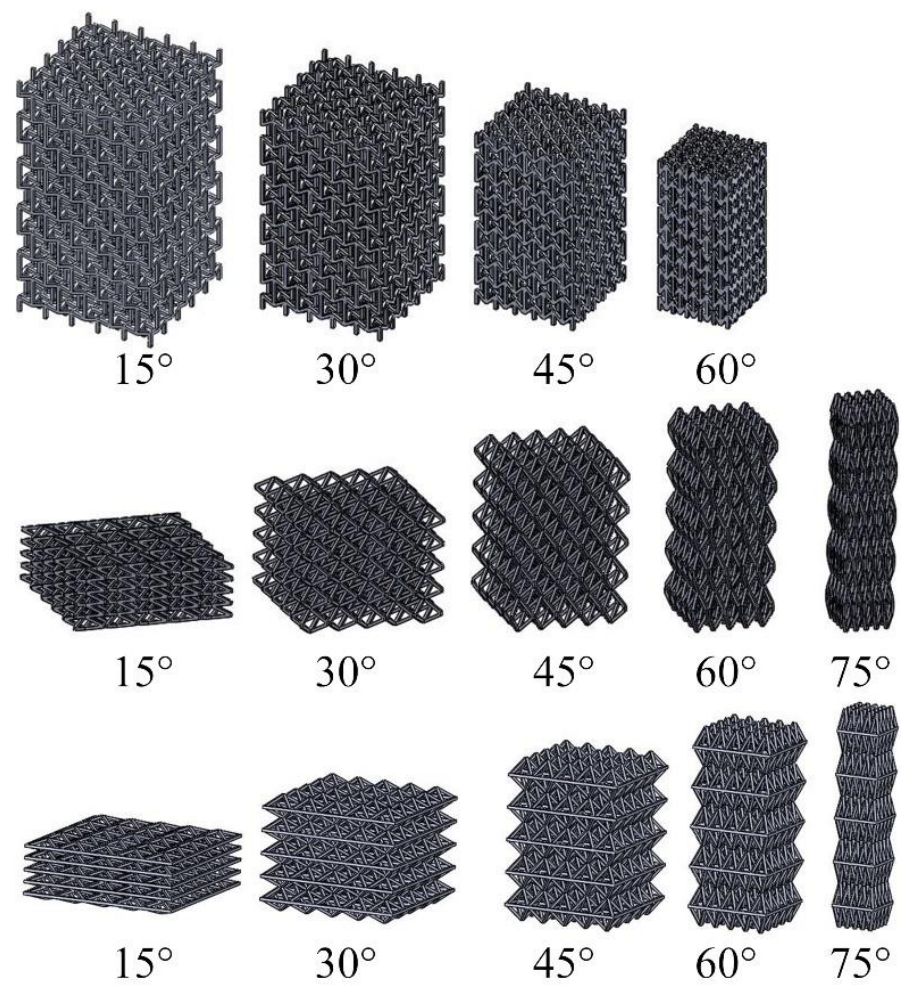

Fig. 4. 4 Different cellular designs with different strut angles varying from $15^{\circ}$ to $75^{\circ}$

As shown in Table 4.2, the change of the strut orientation angles in these cellular structures (Fig. 4.4) resulted in various levels of relative densities. The relative density of both BCC and octahedral structures first decreases and then increases when the strut angle varies from $15^{\circ}$ to $75^{\circ}$ due to their topological symmetry at $45^{\circ}$ strut orientation angle. In comparison, the relative density of auxetic structure keeps increasing as the strut angle increases. In the effort to exclude the effect of relative density from the comparison, the mechanical properties were normalized by their relative densities following the power functions by classic Gibson-Ashby theories, or:

$$
\begin{gathered}
E_{N}=\frac{E}{\rho_{r}^{2}} \\
\sigma_{N}=\frac{\sigma}{\rho_{r}^{3 / 2}}
\end{gathered}
$$


where $E_{N}$ and $\sigma_{N}$ are the relative density-normalized elastic modulus and strength, respectively, $E$ and $\sigma$ are the calculated elastic modulus and strength of the cellular structures, and $\rho_{r}$ is the relative density.

Table 4. 2 Relative density for different structures with different angles

\begin{tabular}{cccccc}
\hline \multirow{2}{*}{ Design } & \multicolumn{5}{c}{ Relative density } \\
\cline { 2 - 6 } & $15^{\circ}$ & $30^{\circ}$ & $45^{\circ}$ & $60^{\circ}$ & $75^{\circ}$ \\
\hline Auxetic & 0.0896 & 0.122 & 0.201 & 0.502 & \\
BCC & 0.106 & 0.075 & 0.079 & 0.131 & 0.262 \\
Octahedral & 0.138 & 0.089 & 0.088 & 0.142 & 0.321 \\
\hline
\end{tabular}

Table 4.3 summarizes the results of the calculated normalized compressive failure strength and normalized Young's modulus of different cellular structure designs with both isotropic and anisotropic material properties. As expected, for all three cellular designs, the mechanical properties reduce as the strut orientation angle increases, which resonates the trend observed with the single struts. It is obvious that the tilted struts contribute significantly to the overall structural response of these cellular structures under uniaxial compressive/tensile stress. To further elucidate the anisotropic material property effect, the property anisotropic ratios, which is defined as the ratio between the mechanical property of a design of anisotropic material versus that of isotropic material $\left(\Omega_{A} / \Omega_{I}\right)$ based on Table 4.3, are illustrated in Fig. 4.5 and Fig. 4.6.

Table 4. 3 Relative density-normalized compressive strength and Young's modulus of cellular designs with different strut orientations under isotropy (I) and anisotropy (A) conditions

$\begin{array}{ll}\text { Unit type } \quad \text { Strength } \sigma_{N}(\mathrm{MPa}) & \text { Young's modulus } E_{N}(\mathrm{GPa})\end{array}$




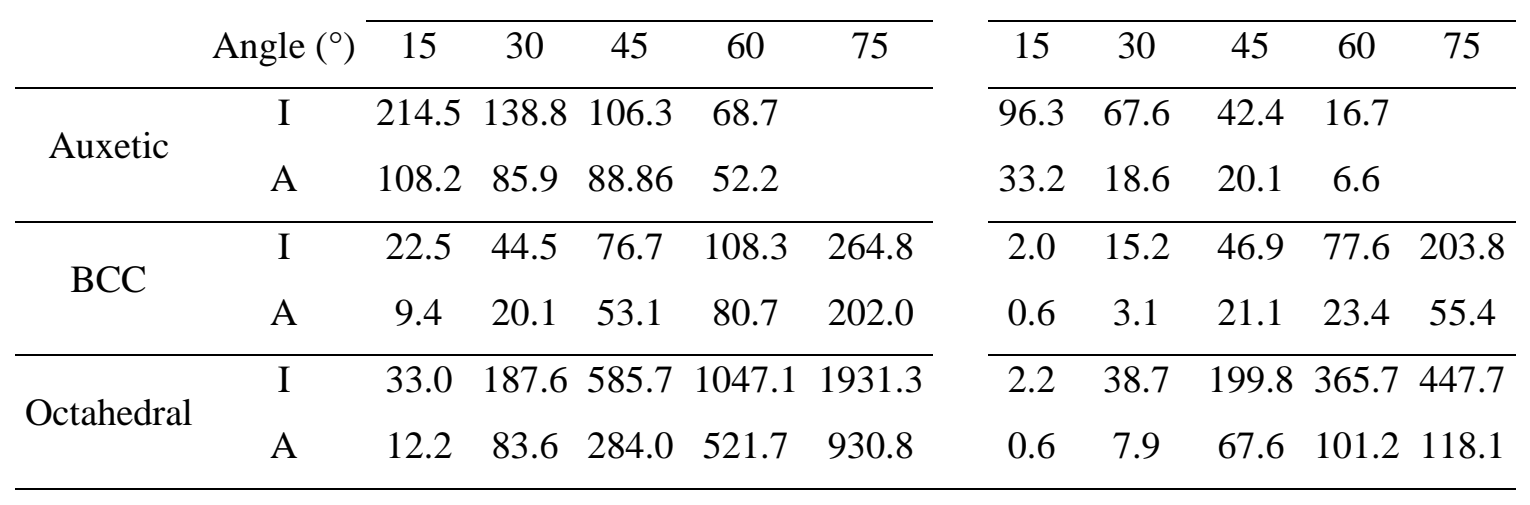

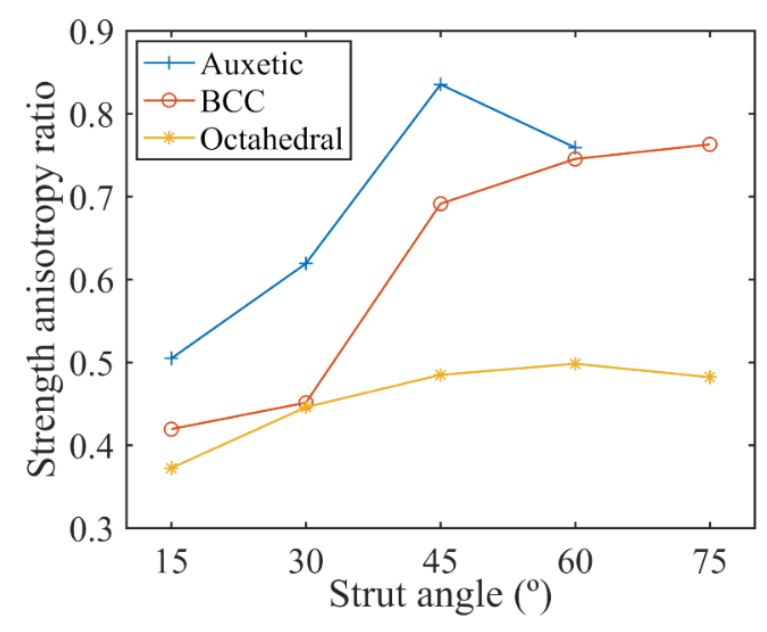

Fig. 4. 5 The strength anisotropy ratio of cellular structures under different strut angles

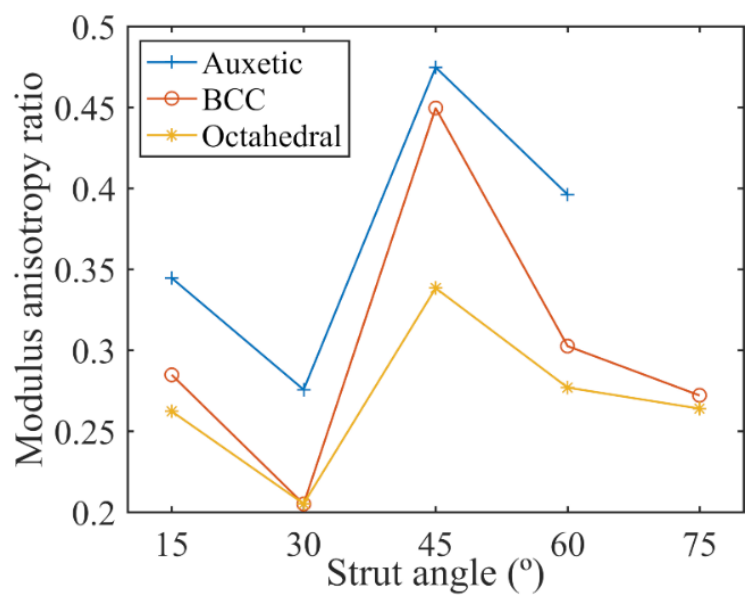

Fig. 4. 6 The modulus anisotropy ratio of cellular structures under different strut angles

From Fig.4.5, the octahedral structures and the re-entrant auxetic structure exhibit the lowest and highest strength anisotropic ratios at all levels of strut orientation angles. 
On the other hand, the octahedral structure exhibits the smallest strength variations as strut orientation varies. It was also noted that the auxetic structures exhibit an obvious nonmonotonous strut orientation angle-strength relationship, which appears to achieve maximum strength at strut orientation angle of $45^{\circ}$. From Fig. 4.6, the auxetic structure exhibits the highest elastic modulus anisotropic ratio, while the octahedral structure exhibits the lowest. It is also notable that all three types of structures exhibit similar strut orientation angle-elastic modulus trend, which exhibits a peak value at strut orientation angle of $45^{\circ}$. Such relationships of all the structures follow the patterns that closely resemble that of the single struts (Fig. 4.3(b)). This can be readily explained by the fact that the elastic behaviors, or deformation, of these structures are dominated by the tilted struts within each types of structures, which themselves in turn exhibit elastic modulus anisotropy as shown in Fig. 4.3(b). On the other hand, the slight difference among different structures could likely be attributed to the vertical or horizontal struts that are present in the auxetic and octahedral structures (Fig. 4.7), which do not exhibit material anisotropy due to the design setting in this study, and therefore act as either stiffening or softening components within the structures depending on their orientation angles.

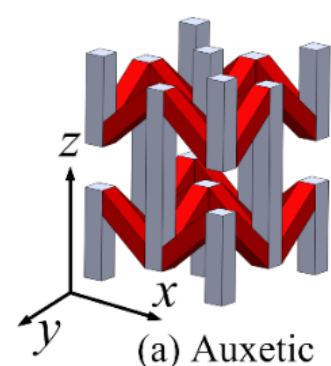

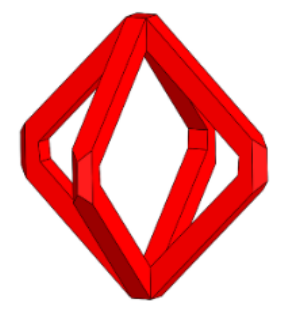

(b) $\mathrm{BCC}$

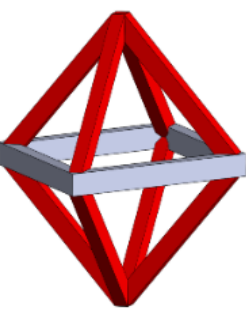

(c) Octahedral

Fig. 4. 7 Different strut types within each type of cellular unit cell (red indicates ones that are subjected to anisotropic material effect, assuming consistent build orientation (z). (a) 
auxetic structure with vertical and inclined struts. (b) BCC structure with inclined struts. (c) octahedral structure with horizontal and inclined struts.

On the other hand, the anisotropic ratios of strength for the three types of cellular structures are less consistent, with re-entrant auxetic structures appearing to exhibit reduced impact from anisotropic material, and octahedral structures appearing to exhibit amplified impact from anisotropic material. The different strength anisotropic ratio trends among the three structures are largely attributable to their topology designs. To obtain more insights into this, consider Equation (4.3) and (4.4) for the principal stress $\left(\sigma_{\text {principal }}\right)$ of the most critical walls within the cellular structure and the nominal stress applied to the whole structure $\left(\sigma_{\text {applied }}\right)$, which describe the relationships between the nominal strain of the structure $(\varepsilon)$ and the corresponding stresses at both structural and individual wall levels for perfectly-elastic cellular structures. In Equation (4.3) and (4.4), $C_{1}$ and $C_{2}$ are scaling factors determined mainly by the cellular topology and pattern designs, and the elastic modulus of the solid materials. Therefore, these two factors can be conveniently interpreted as "microscopic" and "macroscopic" elasticity, respectively. As the failure of the structure is dictated by the failure of the most critical wall within it, i.e. when $\sigma_{\text {principal }}=\sigma_{\text {strength }}$, the maximum strength of the cellular structure $\sigma$ can be obtained by reorganizing Equation (4.3) and (4.4) into Equation (4.5).

$$
\begin{gathered}
\sigma_{\text {principal }}=C_{1} * \varepsilon \\
\sigma_{\text {applied }}=C_{2} * \varepsilon \\
\sigma=\left(C_{2} / C_{1}\right) * \sigma_{\text {strength }}
\end{gathered}
$$

From Equation (4.5), the factors that influence the strength of the cellular structures include the material elastic modulus-dominated part, $C_{2} / C_{1}$, and the material strength- 
dominated part, $\sigma_{\text {strength }}$. With varying material anisotropy effect, the values of $C_{1}$ and $C_{2}$ would vary, which can be quantified from the analytical model and subsequently provide insights into the relative contributions of both material elastic modulus and material strength factors to the strength of the structure with material anisotropy.

For the BCC structures, all the struts are oriented at same inclined angle, and therefore it was expected that the mechanical properties of the structures would exhibit approximately linear correspondence to the strut material properties, as was observed from Fig. 4.5 and Fig. 4.6. This is further clarified via Equation (4.3) to Equation (4.5), as shown in Table 4.4. From Table 4.4, the values of $C_{1}$ and $C_{2}$ for the BCC structures of different strut orientation angle designs under both isotropic (baseline) and anisotropic material conditions are listed. The micro-elasticity anisotropy ratio, or $C_{1 \text { (anisotropic) }} / C_{1 \text { (isotropic), and }}$

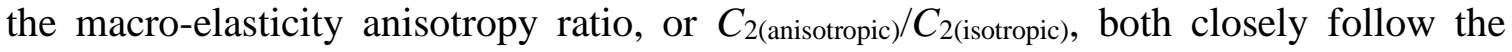
material elastic modulus anisotropy ratio (Fig.4.3b). This clearly shows that the elastic behaviors of the BCC structures at both strut and structural levels are almost entirely determined by anisotropic elastic modulus of the material. This also leads to the result that the material elastic modulus-dominated factor in Equation (4.5), $C_{2} / C_{1}$, remains largely consistent regardless of the strut orientation angle. As a result, the anisotropic strength characteristic of the BCC structures is primarily influenced by the anisotropic strength of the material, $\sigma$ (or $\left.\sigma_{\text {(anisotropic) }} / \sigma_{\text {(isotropic) }}\right)$.

Table 4. 4 Different factors for the strength/modulus anisotropy ratio of BCC structures

\begin{tabular}{|c|c|c|c|c|c|c|c|}
\hline $\begin{array}{c}\text { Orientation } \\
\text { angle/material } \\
\text { type }\end{array}$ & $C_{1}$ & $C_{2}$ & $\begin{array}{c}\text { Critical } \\
\text { strut type }\end{array}$ & $\begin{array}{c}\text { Micro- } \\
\text { elasticity } \\
\text { ratio }\end{array}$ & $\begin{array}{c}\text { Macro- } \\
\text { elasticity } \\
\text { ratio }\end{array}$ & $\begin{array}{c}\text { Material } \\
\text { elastic } \\
\text { modulus ratio }\end{array}$ & $\begin{array}{c}\text { Material } \\
\text { strength ratio } \\
\sigma_{\text {(anisotropic) }} / \\
\sigma_{\text {(isotropic) }}\end{array}$ \\
\hline
\end{tabular}




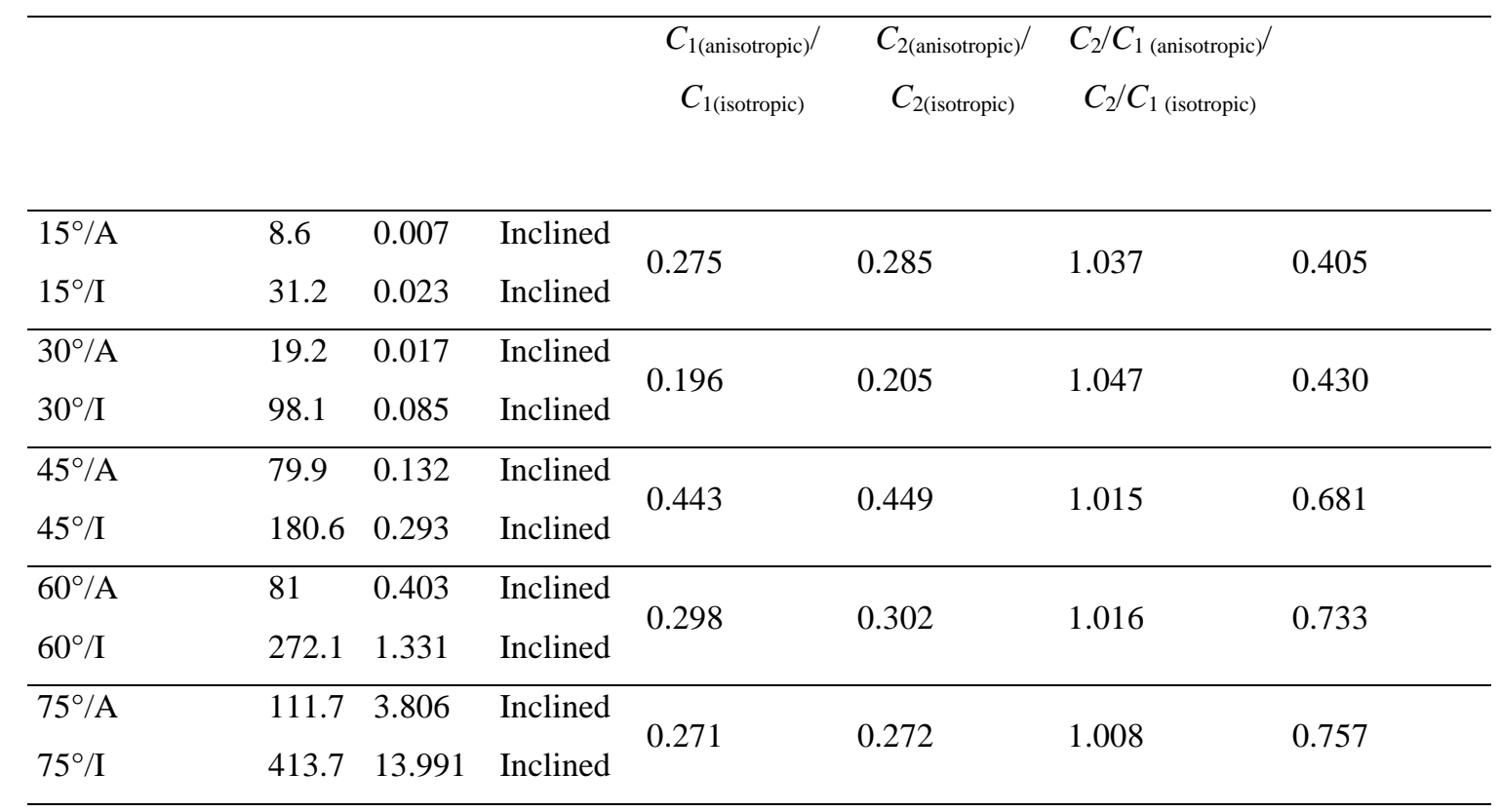

For the auxetic structures, there exist two types of struts, namely the inclined and vertical struts, as shown in Fig. 4.7(a). With fixed build orientation, the inclined struts are subjected to anisotropic material property effects with varying designs, while the vertical struts are unaffected. As a result, the relative criticality of the two types of struts also vary with different anisotropic material effects. As shown in Table 4.5, for the designs investigated in this study, the inclined struts are critical for the designs with very low orientation angles, whereas the vertical struts are critical for the designs with higher orientation angles. With the $15^{\circ}$-orientation angle design and likely other low-orientation angle designs, as the inclined struts exhibit anisotropic material-dependent elasticity and strength, the strength of the structure is influenced by both the elastic modulus and strength of the anisotropic material. Furthermore, due to the mechanical contribution of the vertical struts, the macro-elasticity anisotropy ratio and the micro-elasticity anisotropy ratio follow different dependency functions on the design orientation angle. Therefore, the orientation angle-strength effect is not expected to follow either of the material anisotropy trend as shown in Fig. 4.7(b). On the other hand, with the high-orientation angle designs (e.g. $30^{\circ}-$ 
$60^{\circ}$ designs in this study), as the mechanical properties of the critical struts, i.e. vertical struts, remain unchanged with different designs, the material strength-induced factor in Equation (4.5), $\sigma_{\text {strength }}$, remains constant. Consequently, the anisotropic structural strength becomes entirely influenced by the material elastic modulus-induced factor, $C_{2} / C_{1}$, as elucidated in Table 4.5. Such characteristic also explains the occurrence of maximum strength at $45^{\circ}$-orientation angle design with the auxetic structures, since in this study the elastic modulus of struts exhibits a local maximum at $45^{\circ}$-orientation angle (Fig. 4.7(b)).

Table 4. 5 Different factors for the strength/modulus anisotropy ratio of auxetic structures

\begin{tabular}{|c|c|c|c|c|c|c|c|}
\hline $\begin{array}{c}\text { Orientation } \\
\text { angle/material } \\
\text { type }\end{array}$ & $C_{1}$ & $C_{2}$ & $\begin{array}{l}\text { Critical } \\
\text { strut } \\
\text { type }\end{array}$ & $\begin{array}{c}\text { Micro- } \\
\text { elasticity ratio } \\
C_{1 \text { (anisotropic) }} / \\
C_{1 \text { (isotropic) }}\end{array}$ & $\begin{array}{c}\text { Macro- } \\
\text { elasticity } \\
\text { ratio } \\
C_{2 \text { (anisotropic) }} / \\
C_{2 \text { (isotropic) }}\end{array}$ & $\begin{array}{c}\text { Material } \\
\text { elastic } \\
\text { modulus ratio } \\
C_{2} / C_{1} \\
\text { (anisotropic) } / \\
C_{2} / C_{1} \text { (isotropic) }\end{array}$ & $\begin{array}{l}\text { Material strength } \\
\text { ratio } \\
\sigma_{\text {(anisotropic) }} / \sigma_{\text {(isotropic) }}\end{array}$ \\
\hline $\begin{array}{l}15^{\circ} / \mathrm{A} \\
15^{\circ} / \mathrm{I}\end{array}$ & $\begin{array}{c}39 \\
141.1\end{array}$ & $\begin{array}{l}0.266 \\
0.773\end{array}$ & $\begin{array}{l}\text { Inclined } \\
\text { Inclined }\end{array}$ & 0.276 & 0.345 & 1.247 & 0.405 \\
\hline $\begin{array}{l}30^{\circ} / \mathrm{A} \\
30^{\circ} / \mathrm{I}\end{array}$ & $\begin{array}{c}66.5 \\
178.5\end{array}$ & $\begin{array}{l}0.277 \\
1.006\end{array}$ & $\begin{array}{l}\text { Vertical } \\
\text { Vertical }\end{array}$ & 0.372 & 0.276 & 0.74 & 0.836 \\
\hline $\begin{array}{l}45^{\circ} / \mathrm{A} \\
45^{\circ} / \mathrm{I}\end{array}$ & $\begin{array}{c}89.3 \\
187.9\end{array}$ & $\begin{array}{l}0.814 \\
1.714\end{array}$ & $\begin{array}{l}\text { Vertical } \\
\text { Vertical }\end{array}$ & 0.475 & 0.475 & 1 & 0.836 \\
\hline $\begin{array}{l}60^{\circ} / \mathrm{A} \\
60^{\circ} / \mathrm{I}\end{array}$ & $\begin{array}{c}78.8 \\
180.5\end{array}$ & $\begin{array}{l}1.665 \\
4.204\end{array}$ & $\begin{array}{l}\text { Vertical } \\
\text { Vertical }\end{array}$ & 0.436 & 0.396 & 0.908 & 0.836 \\
\hline
\end{tabular}

For the octahedral structures, there are also two different types of struts (inclined and horizontal struts), as shown in Fig. 4.7(c). As shown in Table 4.6, the micro- and macro-elasticity ratios of the octahedral structures appear to be smaller compared to both the auxetic and BCC structures, which should be attributed by the low mechanical properties of the horizontal struts. With low-orientation angle designs $\left(15^{\circ}-45^{\circ}\right.$ in this study), the inclined struts are more critical, and the strength of the structures are strongly 
correlated to both the elastic modulus and strength of the anisotropic material. With highorientation angle designs $\left(60^{\circ}-75^{\circ}\right.$ in this study), the vertical struts are more critical. As the vertical struts exhibit constant strength, the material strength-induced factor $\sigma_{\text {strength }}$ for octahedral structure remains constant, and the strength of the octahedral structures is mainly influenced, to a relatively small extent, by material elastic modulus-induced factor, $C_{2} / C_{1}$. As octahedral structure exhibit stretch-dominated deformation behaviors, the bending of struts plays less significant role in the overall deformation of the structures. Therefore, the strength of the high-orientation angle octahedral designs exhibits much less anisotropy.

Table 4. 6 Different factors for the strength/modulus anisotropy ratio of octahedral structures

\begin{tabular}{|c|c|c|c|c|c|c|c|}
\hline $\begin{array}{c}\text { Orientation } \\
\text { angle/material } \\
\text { type }\end{array}$ & $C_{1}$ & $C_{2}$ & $\begin{array}{l}\text { Critical } \\
\text { strut type }\end{array}$ & $\begin{array}{c}\text { Micro- } \\
\text { elasticity } \\
\text { ratio } \\
C_{1 \text { (anisotropic) }} / \\
C_{1 \text { (isotropic) }}\end{array}$ & $\begin{array}{c}\text { Macro- } \\
\text { elasticity } \\
\text { ratio } \\
C_{2 \text { (anisotropic) }} \\
C_{2 \text { (isotropic) }}\end{array}$ & $\begin{array}{c}\text { Material } \\
\text { elastic } \\
\text { modulus ratio } \\
C_{2} / C_{1 \text { (anisotropic) }} / \\
C_{2} / C_{1 \text { (isotropic) }}\end{array}$ & $\begin{array}{c}\text { Material } \\
\text { strength ratio } \\
\sigma_{\text {(anisotropic) }} / \\
\sigma_{\text {(isotropic) }}\end{array}$ \\
\hline $\begin{array}{l}15^{\circ} / \mathrm{A} \\
15^{\circ} / \mathrm{I}\end{array}$ & $\begin{array}{c}7.3 \\
25.7\end{array}$ & $\begin{array}{l}0.011 \\
0.041\end{array}$ & $\begin{array}{l}\text { Inclined } \\
\text { Inclined }\end{array}$ & 0.285 & 0.262 & 0.92 & 0.405 \\
\hline $\begin{array}{l}30^{\circ} / \mathrm{A} \\
30^{\circ} / \mathrm{I}\end{array}$ & $\begin{array}{l}12.8 \\
64.7\end{array}$ & $\begin{array}{l}0.063 \\
0.307\end{array}$ & $\begin{array}{l}\text { Inclined } \\
\text { Inclined }\end{array}$ & 0.198 & 0.205 & 1.035 & 0.430 \\
\hline $\begin{array}{l}45^{\circ} / \mathrm{A} \\
45^{\circ} / \mathrm{I}\end{array}$ & $\begin{array}{c}50.5 \\
106.3\end{array}$ & $\begin{array}{l}0.524 \\
1.547\end{array}$ & $\begin{array}{l}\text { Inclined } \\
\text { Inclined }\end{array}$ & 0.475 & 0.338 & 0.712 & 0.681 \\
\hline $\begin{array}{l}60^{\circ} / \mathrm{A} \\
60^{\circ} / \mathrm{I}\end{array}$ & $\begin{array}{c}26.7 \\
138.2\end{array}$ & $\begin{array}{l}2.042 \\
7.374\end{array}$ & $\begin{array}{l}\text { Horizontal } \\
\text { Horizontal }\end{array}$ & 0.193 & 0.277 & 1.433 & 0.348 \\
\hline $\begin{array}{l}75^{\circ} / \mathrm{A} \\
75^{\circ} / \mathrm{I}\end{array}$ & $\begin{array}{c}26.2 \\
137.9\end{array}$ & $\begin{array}{l}12.173 \\
46.128\end{array}$ & $\begin{array}{l}\text { Horizontal } \\
\text { Horizontal }\end{array}$ & 0.19 & 0.264 & 1.386 & 0.348 \\
\hline
\end{tabular}

In considering the strength of the cellular structures, both elastic modulus and strength effects of materials should be considered. If the critical struts exhibit low elastic 
modulus, the macro-elasticity ratio $\left(C_{2 \text { (anisotropic) }} / C_{2 \text { (isotropic) }}\right)$ tend to be higher than the micro-

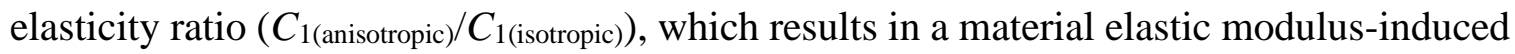
factor of $>1$. A higher material elastic modulus-induced factor indicates that the elasticity of the structure is enhanced by the other non-critical struts, which could potentially provide useful design information for these cellular structures.

\subsubsection{Effect of the pattern size on the anisotropic structural mechanical properties}

Due to the significant effect on stress distribution and deformation of the struts, the pattern size of the cellular structures was considered in this study in order to provide comprehensive view to the problem. For pattern size effect study, 7 different pattern sizes with unit cell numbers ranging from 2 to $8(2 \times 2 \times 2,3 \times 3 \times 3,4 \times 4 \times 4,5 \times 5 \times 5,6 \times 6 \times 6,7 \times 7 \times 7$, $8 \times 8 \times 8$ ) for each types of cellular designs were investigated. For all the designs, the orientation angle of the titled struts was set to be $45^{\circ}$. All the other geometrical parameters remain the same as the previous study. 

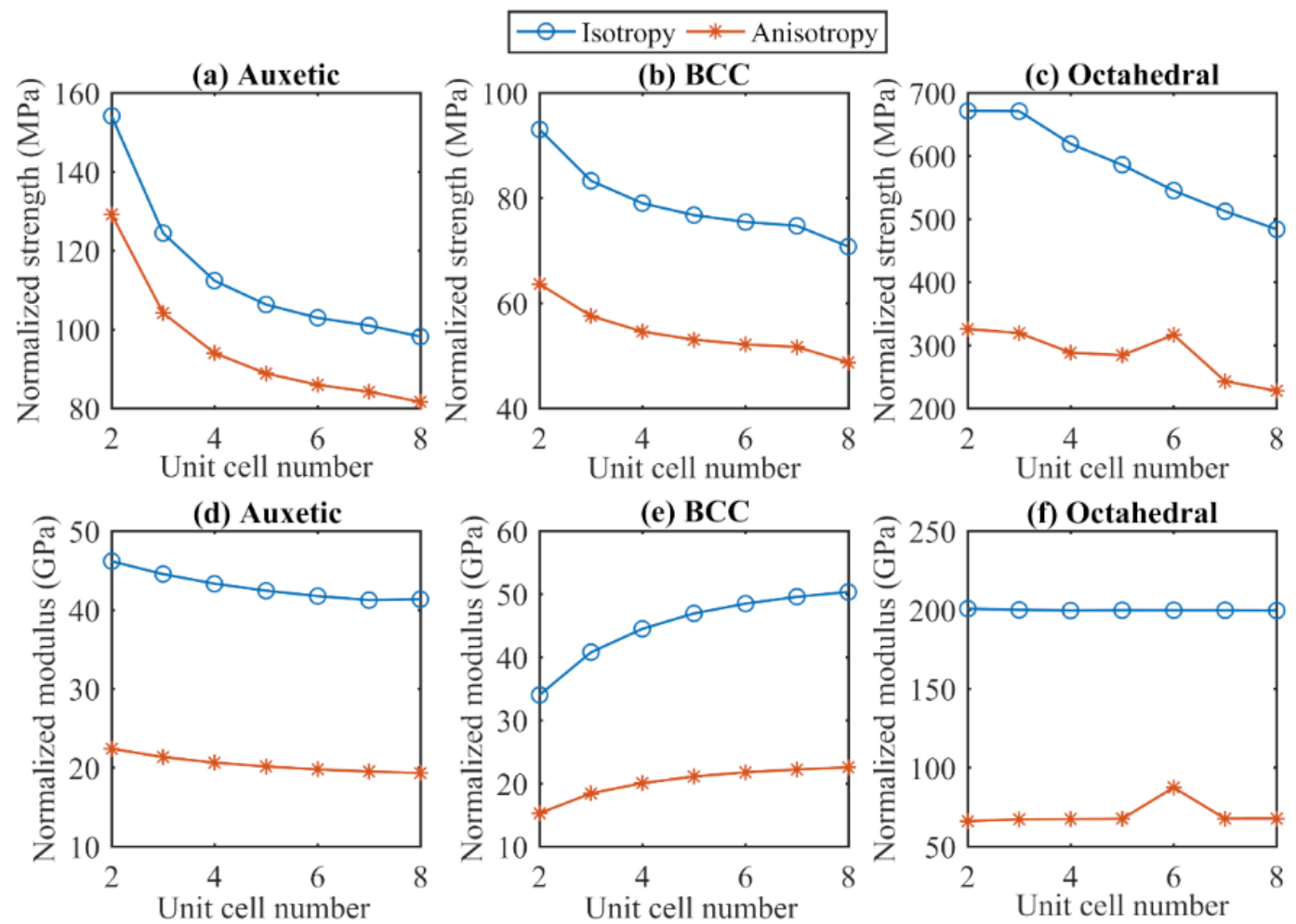

Fig. 4. 8 The relationship between relative density-normalized compressive strength/Young's modulus and unit cell numbers of different cellular structures: (a-c) normalized strength of three structures; (d-f) normalized modulus of three structures

Fig. 4.8 shows the relationships between the relative density-normalized compressive strength/Young's modulus of the cellular patterns and their pattern sizes for the three types of cellular designs. From the results, cellular structures of both isotropic and anisotropic materials exhibit similar pattern size effects, which indicates that pattern size effect could likely be decoupled from material anisotropy effect during the design process. For structural strength, all three types of cellular designs exhibit some levels of pattern size effects. In addition, the observed trends also contradict to the conclusions from classic literatures, which suggests that the strength of the cellular structures increase with increased pattern sizes [129][130]. From this work, with increased pattern sizes, the 
strengths of all three types of cellular designs decrease, indicating that the reduced relative boundary effects actually correspond to the "weakening" of the cellular structures. Without further analysis, it was speculated that this was caused by the reduced interaction of the boundary constraining effects from the two opposite sides as the pattern size increases. Considering the non-homogenization-based modeling approach of this work, such nonconventional observations also suggest the possibility of the need of major revision of the modeling approach with cellular structures. On the other hand, it was also noted that these observations agree well with some literatures of experiment-based studies [131][132].

For the elastic modulus, all three types of cellular designs exhibit different trends. For auxetic structure, the elastic modulus of the structure slightly decreases as the pattern size increases, whereas for the BCC structure, the opposite trend was observed. These observations are consistent with earlier experimental observations [119][133]. On the other hand, the octahedral structure exhibits little pattern size effects. Among the three designs, the pattern size effects appear to be most significant for the BCC structures, which undergo more significant property changes (a strength decrease of $\sim 23 \%$ and a modulus increase of $\sim 48 \%$ ) as the pattern size increases from 2 to 8 .

To further investigate the potential coupling effect between pattern size and material anisotropy, Table 4.7 summarizes the average strength/modulus anisotropy ratio of the three types of designs averaged over all pattern sizes. From the table, both the strength and modulus anisotropy ratio are highly consistent for each types of cellular designs, which confirms that for strength design the two effects can be largely decoupled. It should also be noted that as only $45^{\circ}$-orientation angle designs were investigated, 
conclusions about the comparison of strength and elastic modulus among different designs should not be cursively made.

Table 4. 7 Average strength/modulus anisotropy ratio of different structures

\begin{tabular}{ccc}
\hline Cellular design & Strength anisotropy ratio & Modulus anisotropy ratio \\
\hline Auxetic & $83.52 \% \pm 0.26 \%$ & $47.56 \% \pm 0.56 \%$ \\
BCC & $69.08 \% \pm 0.51 \%$ & $44.94 \% \pm 0.14 \%$ \\
Octahedral & $49.30 \% \pm 4.22 \%$ & $35.11 \% \pm 3.83 \%$ \\
\hline
\end{tabular}

In addition, due to the varying boundary-induced stress concentration effects, the pattern size was expected to play more significant role in influencing the location of the critical struts. To better illustrate this effect, the stress distributions of the three cellular structures of different pattern sizes were visualized using FEA tool, which yield essentially the same results as the analytical models. The FEA simulations were performed with SolidWorks Simulation, which was selected due to its integration with SolidWorks that was also used for the creation of the cellular structure models for experimentation. Static elastic mechanical analysis was performed for the elastic response of the structures. Convergence analysis was conducted to determine the final mesh size setting. The boundary conditions for the simulation was shown in Fig. 2.9(a). FEA analysis for structures of both isotropic and anisotropic material properties were carried out using the previously discussed input variables.

For the BCC structures, the most critical struts are always located at the center of the structures. Due to the boundary constraints, the stress distribution of the BCC structures exhibits highly regular stress concentration patterns across the volumetric diagonal lines, which was previously investigated [132]. The consistent anisotropy effect, as well as the 
regular stress concentration characteristics, results in high regularity of critical strut locations of the BCC structures, and therefore is not further elaborated.

Fig. 4.9 illustrates stress distributions and structural deformation of the auxetic structures of different pattern size with both isotropy and anisotropy materials. The two orientation levels, $45^{\circ}$ and $15^{\circ}$, which represent the cases with different critical strut types (Table 4.5), were included in the illustration. The results clearly illustrate the presence of the boundary-affected regions of the structures, which span roughly half a layer at each constrained boundary for the auxetic structures. With increased pattern size, the boundaryaffected regions remain relatively limited to half a layer, while the regions the are less affected by boundaries increase. In addition, the stress distributions within these less affected regions are also highly regular. This corresponds to the common notion of the small size effect of auxetic structures. Furthermore, there also exist low-stress strips along the unconstrained edges, which clearly shows the effect of free surface boundary condition. With isotropic material properties, due to the stress concentration effects at the boundaries, the critical struts are located at the corner of the structures, as circled in red in Fig. 4.9. For the structures with strut orientation angle of $45^{\circ}$, the presence of material anisotropy shifts the locations of critical struts to near the middle section of the edges in the low-boundary effect regions. The shift of critical strut location can be attributed to the increased deformation of the inclined struts due to material anisotropy, which alters the local rigidity and relaxes stress concentration effects at the boundaries. On the other hand, for the structures with strut orientation angle of $15^{\circ}$, the locations of the critical struts remain consistent with material anisotropy. This can be attributed to the combined effect of 
boundary constraint and the further weakening of the critical struts (inclined) in this region due to material anisotropy.

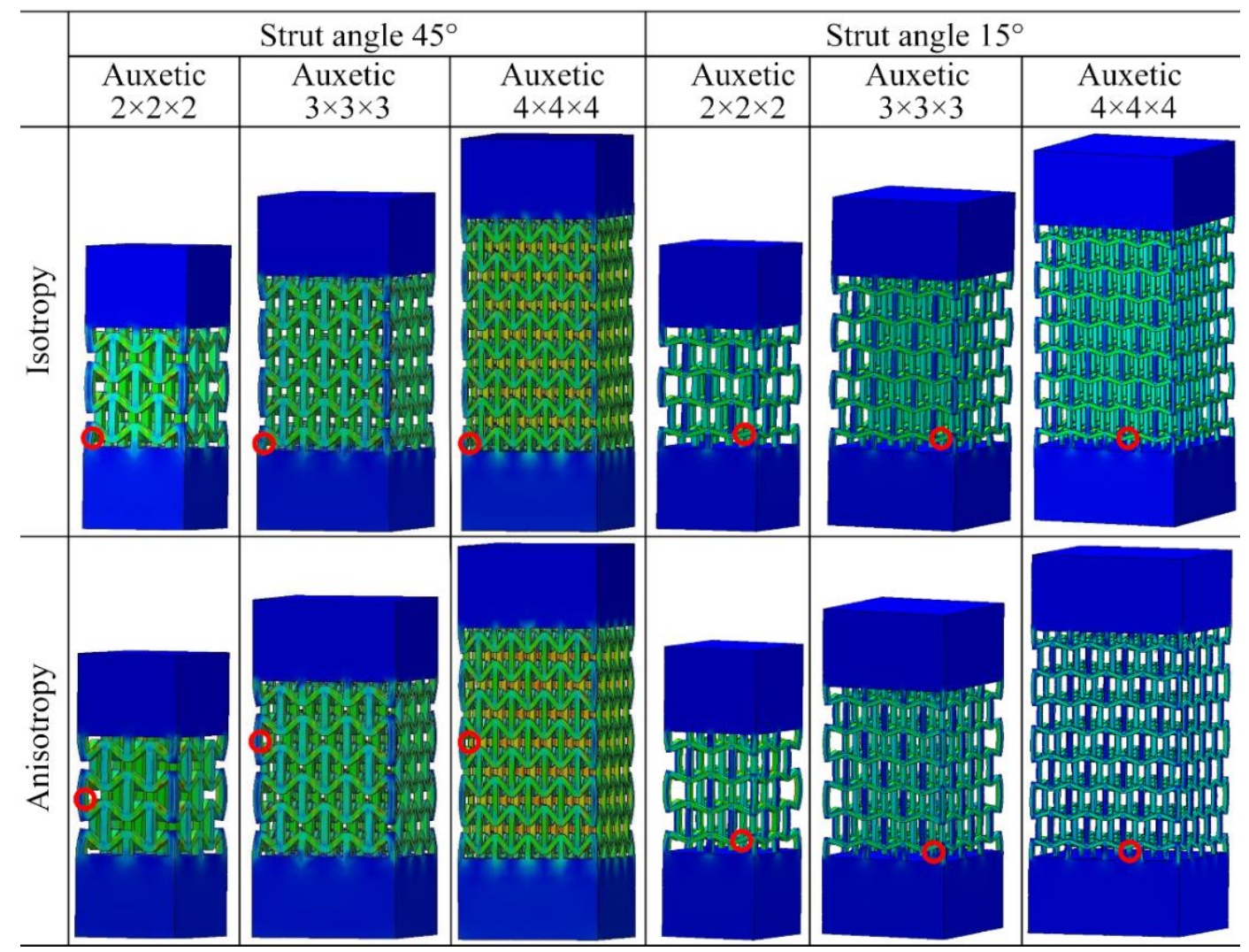

Fig. 4. 9 The stress distribution and structural deformation of the auxetic structures $\left(45^{\circ}\right.$ and $15^{\circ}$ ) with different pattern sizes

Similar analysis is shown in Fig. 4.10 for the octahedral structures of different pattern size with both isotropy and anisotropy materials. The two strut orientation design cases selected, including the $45^{\circ}$ and $60^{\circ}$, again represent cases with two different types of critical struts (Table 4.6). With stretching-dominated deformation characteristic, the struts of the octahedral structures are generally more constrained even with free-surface boundary conditions, which explains the lack of low-stress regions towards the free surfaces. For the octahedral structures, the critical struts are generally located within the internal unit cells 
near the middle sections of the octahedral structures, which is relatively consistent at varying pattern sizes. With material anisotropy effect, the locations of the critical struts remain generally consistent for the octahedral structures, regardless of the critical strut type. Considering the increased criticality of the inclined struts with reduced orientation angles, it could be speculated that a change of critical strut type (and correspondingly location) can be observed at an orientation angle between $45^{\circ}$ and $60^{\circ}$ for the octahedral structures.

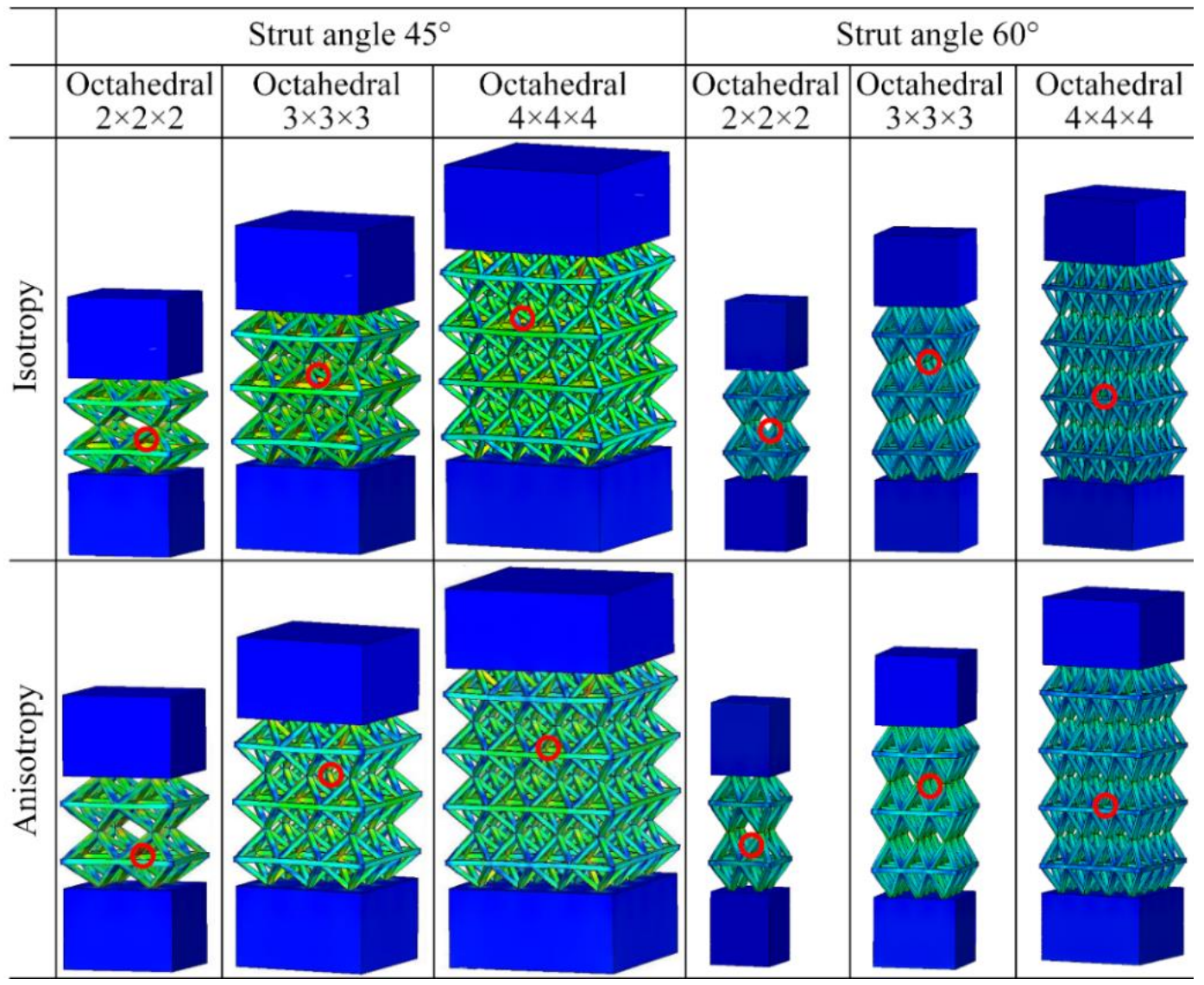

Fig. 4.10 The stress distribution and structural deformation of the octahedral structures $\left(45^{\circ}\right.$ and $\left.60^{\circ}\right)$ with different pattern sizes

As a quick recap, the structure pattern size effect mainly affects the homogeneity of the stress distribution of different regions of the cellular structures, while the material anisotropy affects the stress distribution among different types of struts. Ultimately, the 
strength of the structures is influenced by the interplay of both structural topology designs and the material anisotropy.

Fig. 4.11 and Fig. 4.12 further illustrate the effect of the unit cell pattern size on the anisotropic failure patterns for auxetic and octahedral structures, respectively. As discussed above, the BCC structures only have one type of struts- inclined struts. The material anisotropy only affects its overall strength and modulus. The differences among different strut are not affected. In another words, the relative stress levels among different struts keep the same. Therefore, the failure patterns of the BCC structures will also not be affected by the materials anisotropy, which will not be discussed here.

\begin{tabular}{|c|c|c|c|c|}
\hline & $2 \times 2 \times 2$ & $3 \times 3 \times 3$ & $4 \times 4 \times 4$ & \\
\hline \\
2
\end{tabular}

Fig. 4. 11 Failure patterns of the auxetic structures with different pattern sizes for both isotropic and anisotropic materials 
From Fig. 4.11, it can be seen that the overall failure patterns of the auxetic structures were slightly affected by the material anisotropy. When the pattern size varies from $2 \times 2 \times 2$ to $4 \times 4 \times 4$, the fractures all go from the corner to the center. When the pattern size becomes $5 \times 5 \times 5$, the fractures locate in the top layers. Combining the failure initiation analysis in Fig. 4.9, we can know that the material anisotropy leads to the change of failure initiations when the pattern size changes. These changes further resulted in a slight difference between the failure pattens of isotropic and anisotropic structures.

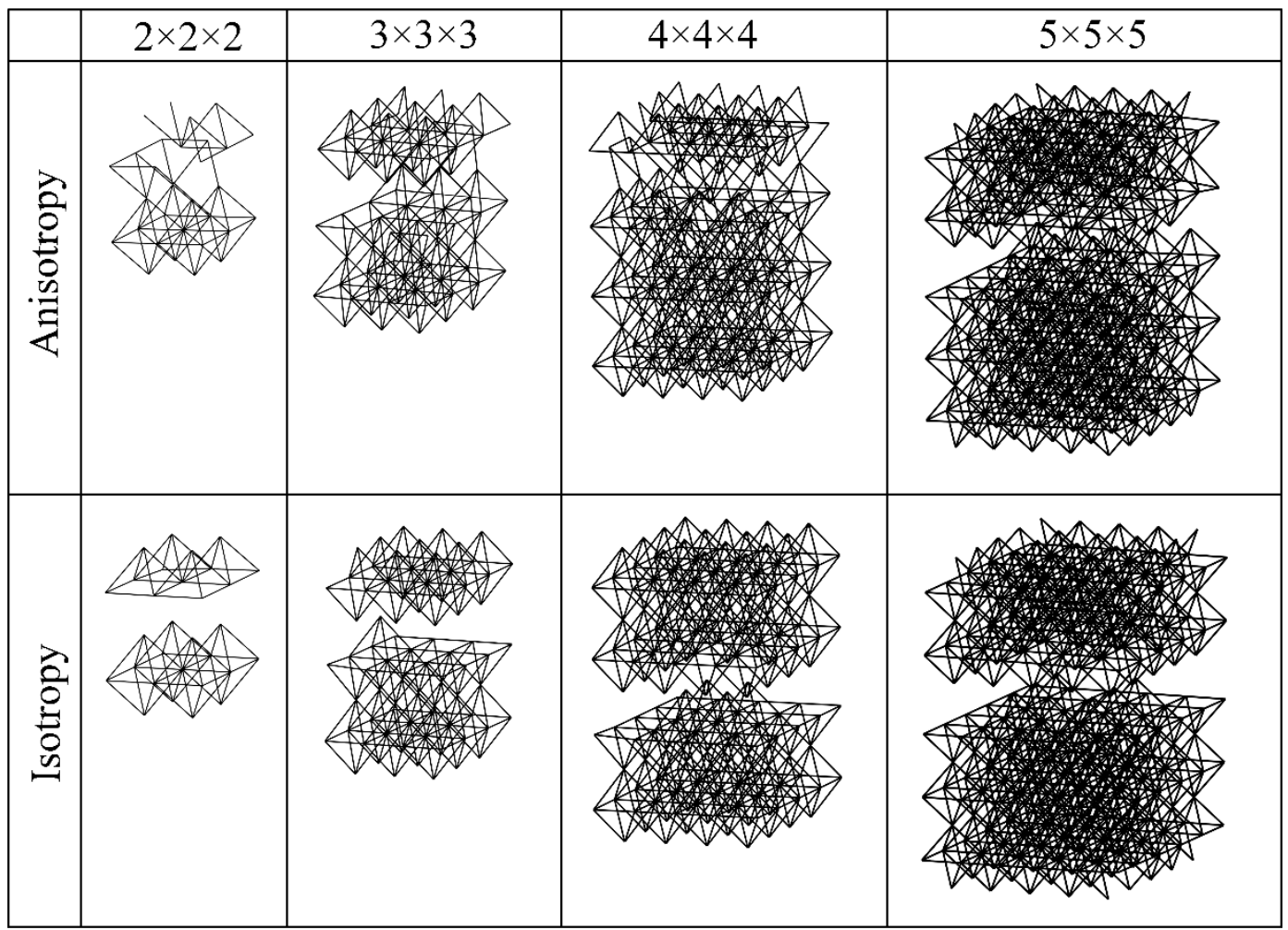

Fig. 4. 12 Failure patterns of the octahedral structures with different pattern sizes for both isotropic and anisotropic materials

Fig. 4.12 shows that failure patterns of the octahedral structures. It can be found that for the isotropic materials, the failure patterns are quite consistent when the pattern size changes. The failure paths were all located in the middle layers. When considering the 
material anisotropy, the failures have a tend to go from the middle layers to the corner layers.

From the failure patten analysis, the material anisotropy not only affects the stress distribution among different types of struts at the initial failures, but only affects the failure paths during the crack propagation.

\subsection{Experimental verification}

$\underline{\text { Table 4. } 8}$ Design parameters for the different cellular structures

\begin{tabular}{ccccccc}
\hline Design & Cell type & $\begin{array}{c}\text { Angle } \\
\left({ }^{\circ}\right)\end{array}$ & $\mathrm{H}(\mathrm{mm})$ & $\mathrm{L}(\mathrm{mm})$ & $\begin{array}{c}\text { Strut } \\
\text { size }(\mathrm{mm})\end{array}$ & $\begin{array}{c}\text { Actual } \\
\text { density }\left(\mathrm{g} / \mathrm{cm}^{3}\right)\end{array}$ \\
\hline Aux1 & Auxetic & 15 & 5 & 3.5 & 1 & $0.597 \pm 0.011$ \\
Aux2 & Auxetic & 30 & 7 & 3.5 & 1 & $0.612 \pm 0.013$ \\
BCC1 & BCC & 30 & -- & 4.5 & 1 & $0.659 \pm 0.008$ \\
BCC2 & BCC & 45 & -- & 6.3 & 1 & $0.390 \pm 0.004$ \\
Octa1 & Octahedral & 45 & -- & 5.4 & 1 & $0.665 \pm 0.006$ \\
Octa2 & Octahedral & 45 & -- & 7.6 & 1 & $0.312 \pm 0.015$ \\
\hline
\end{tabular}

In order to verify the accuracy of the models, two design configurations for each type of cellular structures were randomly designed for experimental study. Table 4.8 shows the design parameters for each configuration. For all the structures, square struts of $1 \mathrm{~mm}$ in dimension were designed. In addition, pattern size of $4 \times 4 \times 4$ was chosen rather arbitrarily for all the structures. All of the samples were fabricated using the same system/material combination (Arcam EB-PBF-S400/AP\&C Ti6Al4V) utilized in the material anisotropy benchmark study. 5 samples of each design were fabricated, and all the samples were fabricated in one build. The fabricated samples of the six design configurations are shown in Fig. 4.13. The actual density of each design (Table 4.8) was calculated through the 
sample mass divided by the geometric bounding volume, which indicates that the quality of fabricated samples is significantly consistent.

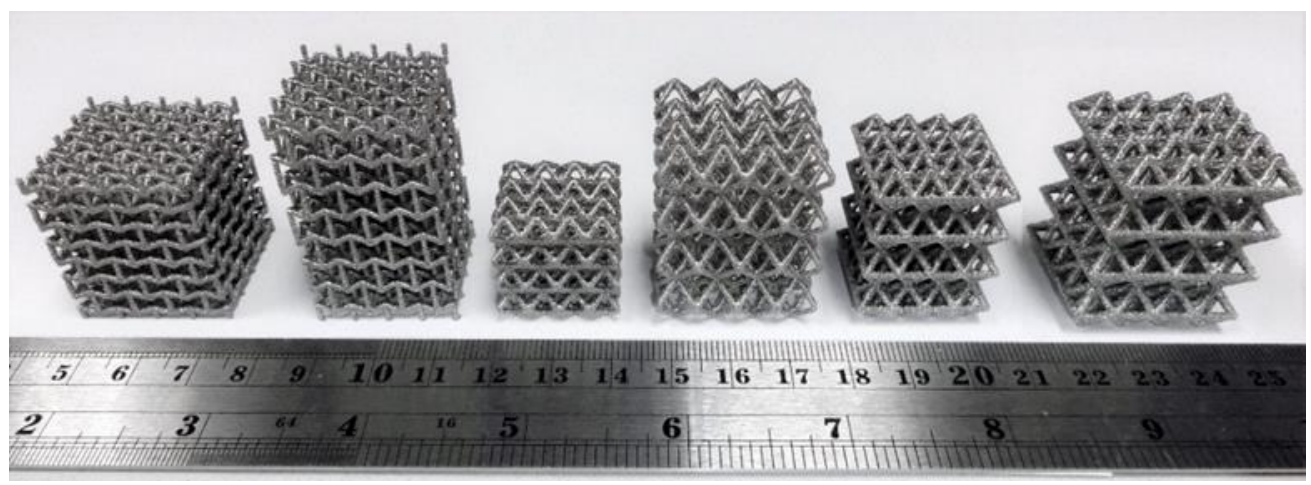

Fig. 4. 13 Fabricated EB-PBF samples

Compression testing was carried out with an Instron 5569A (50kN load cell) testing system at a constant strain rate of $0.3 \mathrm{~mm} / \mathrm{min}$ for all the samples. During the testing, the samples were placed between two tool steel platens and compressed until the total strain achieved over $50 \%$. The stress was obtained from the applied force obtained by the load cell. The compressive strength of the structure was defined as the maximum stress level before the occurrence of the first failure, which also coincide with the maximum stress level that the structure could withstand (subsequent failures occurred at lower stress levels).

The experimental results of the compressive strengths and elastic modulus of all the structures are shown in Fig. 4.14 and Fig. 4.15. Overall, the predictions based on anisotropic material properties were able to provide significantly improved accuracy compared with the ones based on isotropic material properties. For the auxetic structures, the percentage errors of the model for the compressive strength and elastic modulus, which were calculated as the prediction errors as percentages of the experimental values, were $11.5 \%$ and $21.1 \%$ using anisotropic material model, as opposed to $36.8 \%$ and $137.6 \%$ respectively using the isotropic material model. For the BCC structures, the percentage 
errors of compressive strength and elastic modulus were $18.7 \%$ and $25.1 \%$ using the anisotropic material model, as opposed to $78.8 \%$ and $257.7 \%$ using isotropic material model. Lastly, for the octahedral structures, the percentage errors of compressive strength and elastic modulus were $28.2 \%$ and $4.4 \%$ using anisotropic material model, as opposed to $187.1 \%$ and $29.0 \%$ using isotropic material model.

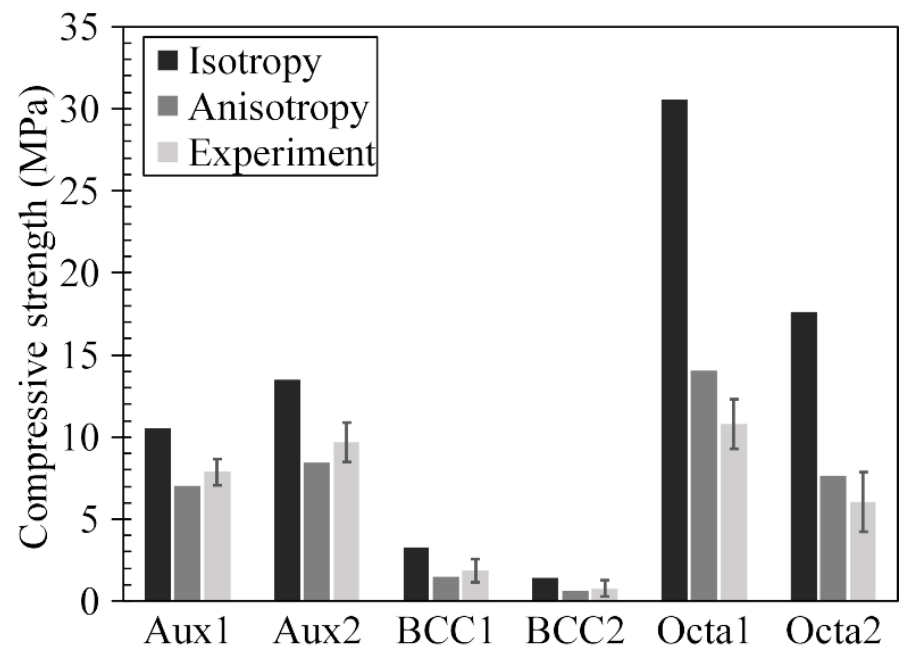

Fig. 4. 14 Compressive strength comparison between the predicted strength and experiment strength

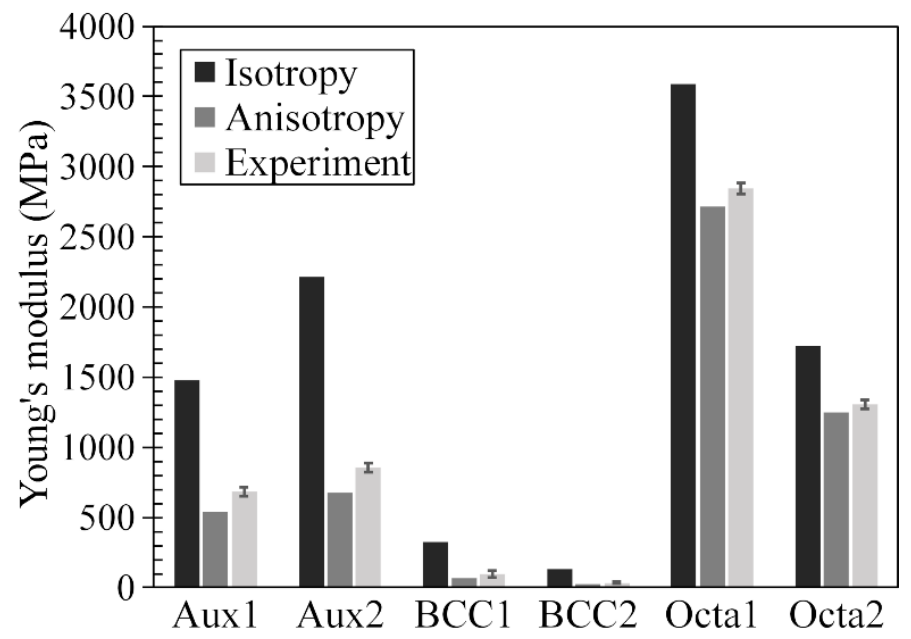

Fig. 4. 15 Elastic modulus comparison between the predicted strength and experiment strength 


\subsection{Conclusions}

In this section, the effects of material anisotropy and structural pattern size on the mechanical properties of three types of 3D cellular designs (auxetic, BCC and octahedral) were analyzed using a direct stiffness matrix-based analytical model using Timoshenko beam theory. Ti6Al4 struts with different orientations $\left(0^{\circ}, 15^{\circ}, 30^{\circ}, 45^{\circ}, 60^{\circ}, 75^{\circ}\right.$ and $\left.90^{\circ}\right)$ respect to the building directions manufactured by the Arcam S400 EB-PBF process were tested to experimentally establish the material anisotropy model of tensile strength and elastic modulus. The predicted mechanical properties of the cellular structures were compared with experimentation using the same material/system combination, and it was verified that the anisotropy model provides a more accurate method for the prediction of the characteristics of the cellular structures. In addition, the modeling-based studies also reveals some additional insights into the heterogeneous mechanical characteristics of these structures under uniaxial static loading. The observations and findings presented in this study are summarized below:

1.The tensile testing results of the single strut with different build orientation angles suggest that there exists significant material anisotropy with both the tensile strength and elastic modulus.

2.The elastic modulus anisotropy of the cellular structures generally follows the elastic modulus anisotropy of the single struts. In addition, as the presence of vertical or horizontal struts act as reinforcement and weakening components respectively, the auxetic structures and octahedral structures exhibit the highest and lowest elastic modulus, respectively. 
3.The strength of the cellular structures is influenced by both the strength and elastic modulus of the struts. Therefore, the anisotropy of the strength of the three types of cellular structures exhibit different characteristics due to their topology designs.

4.All the three types of structures exhibit varying levels of pattern size dependency, with the actual trend differing from many previous conclusions. This highlights the need for adequate consideration of the boundary effects within the cellular structures.

5.For the prediction of mechanical strength and elastic modulus, the pattern size and the material anisotropy are independent factors that can be decoupled during the design; On the other hand, the pattern size appears to have relatively small influence to the location of the critical struts with the designs investigated in this study, although such observation should be subjected to further scrutiny due to the non-negligible stress concentration effects of boundary constraints. 


\section{CHAPTER V}

\section{MODELING OF THE EFFECT OF LOCAL MATERIAL IMPERFECTION TO THE}

STRUCTURAL MECHANICAL PROPERTY VARIABILITY OF 2D FINITE-SIZE

\section{CELLULAR STRUCTURES}

\subsection{Introduction}

For the cellular structures, we know that many of the mechanical characteristics are closely associated with their porosities, which allow for significant amount of local responses of cellular walls or struts such as bending, buckling, twisting and fracture. Aggregately, these local responses give rise to various attractive mechanical properties of the cellular structures. In the design of cellular structures, generally the local heterogeneity of the structures in response to mechanical loading is alleviated via various means. Empirical design equations, often based on relative densities, could be established experimentally for specific types of cellular structures [41][26][68]. This approach is of often highly efficient for practical design purposes and is particularly suitable for traditional cellular foams with stochastic topologies. However, this approach tends to be rather inefficient when the design space becomes large, e.g. when cellular structures with topology control and/or multiple performance objectives are designed. On the other hand, FEA-based modeling can be employed on the full-structure level for comprehensive insights of the behaviors and characteristics of the designs at both local and structural levels, often with minimal loss of accuracy once the FEA model is tuned in [65][66]. However, due to the small dimensional scale of individual struts/ walls in comparison to the entire 
structures, full-scale FEA of cellular structures often involve expensive computations. This issue, further compounded by the need of relatively large number of simulations needed in order to gain adequately design insights, makes FEA-based approaches somewhat inefficient in handling complex design tasks with multiple design objectives, or to search for optimum solutions. To partially circumvent this challenge, homogenization-based modeling was often utilized, which treats the lower-dimensional scale cellular architectures as continuum of equivalent material properties [134][135][136]. For cellular structure designs with periodic unit cell topologies, the homogenization-based approach can be relatively handled by analyzing a unit cell with periodicity boundary conditions. Alternatively, asymptotic homogenization can be employed, which imposes slightly weaker boundary conditions compared to the model with perfectly periodic unit cell but often converges to similar predictions. However, the homogenization treatment inevitably eliminates some information at local cellular wall/strut levels, which sometimes can be problematic.

Recently, multiple works have shown that for finite-size cellular structures, the homogenization approximation might not be applicable [137]. With these structures, due to the relatively small pattern sizes, the boundary constraints such as skins and interfaces exert non-negligible effects to the overall mechanical responses of the structures. As a result, the mechanical properties of the cellular structures could significantly deviate from the ideal ones. This is largely attributed to the non-trivial local structural response effects. The mechanical characteristics of the finite-size cellular structures, therefore, are influenced by both the pattern size and the number of unit cells in principal directions, as well as the unit cell topology designs. This is particularly relevant to AM structural designs, 
as in many instances the resolutions of the AM systems dictate that the cellular structure designs to have relatively large unit cell sizes and small pattern sizes.

Another important factor closely associated with the local mechanical responses of the cellular structures is the variability of mechanical properties of the individual walls and struts caused by the defects discussed in Section 1.2.2. In most of literatures, the defect information was obtained from fabricated samples through $\mu \mathrm{CT}$ scanning which might be restricted by the computational expense and might also be difficult for the understanding of the generic cellular design. On the other hand, these defect investigations focus mainly on the dimensional features. In this case, it is often assumed that the material properties of these features can still be represented by the standard AM material database. This approach can be potentially problematic, as it could be reasonably anticipated that due to the altered process conditions, the microstructures and consequently the material properties of the cellular struts/walls could be different from bulk features. As it can be experimentally difficult to isolate the effect of internal defects from material properties with lightweight features, it might be more efficient in practice to treat these internal defects as "intrinsic" to thin walls and struts [138][139]. Following this approach, the mechanical properties of the lightweight features, such as elastic modulus, ultimate strength and ductility, could be characterized experimentally, which are dependent on not only the process conditions but also the geometry of the features (e.g. thickness) [140][141][142]. So far, relatively little is known about the effect of local mechanical property variability of the finite-size cellular structures on its overall characteristics, which will be discussed in this section.

In this section, a generic and novel method was proposed to model the defects by representing the defects with the cell wall material property variability (the cell wall 
strength variability and cell wall modulus variability) through analytical modeling. The present study focuses on the investigation of the effect of cell wall material property variability on the overall mechanical properties at the structure level. In addition, to account for the effect of non-ideal boundary conditions, the study also includes the investigation of the effect of the number of unit cells in the patterns. To isolate the effect of the material anisotropy caused by the strut build orientation on the mechanical properties, the investigation was carried out with various 2D cellular structures; however, the same methodology is expected to be applicable to 3D designs as well.

\subsection{Modeling for cellular structures with material property variability}

Three different structures were investigated as shown in Fig. 5.1. The choice of these three cellular structures was motivated by two generalized cellular structure design rules: Poisson's ratio (auxetic vs. non-auxetic) and bending-/stretching-dominated deformation mechanism. Fig.1a shows the re-entrant auxetic structure with negative Poisson's ratios and bending-dominated mechanism. The diamond structure (Fig. 5.1(b)) is a non-auxetic design that exhibits bending-dominated mechanism. The triangular structure (Fig. 5.1(c)) is also a non-auxetic design, and it exhibits stretching-dominated mechanism. The geometry design of the re-entrant auxetic structure is characterized by wall thickness $t$, opening angle $\theta$, length of re-entrant wall $L$ and wall height $H$. The geometrical designs of the diamond and triangular structures area characterized by the length of each wall $L$, the opening angle $\theta$ and wall thickness $t$. 


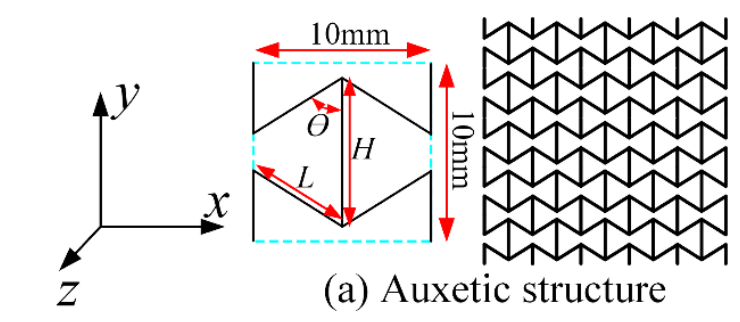

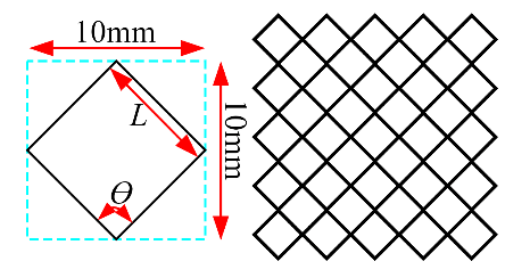

(b) Diamond structure

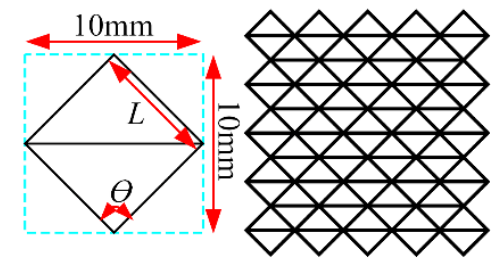

(c) Triangular structure

Fig. 5. 1 The designed cellular structures

Table 5.1 lists the specific geometrical designs of the three types of cellular structures. In addition, for each design, three levels of pattern sizes, or the number of unit cells along both the $x$ and $y$ directions (indicated in Fig. 5.1), including 5×5, $8 \times 8$ and $10 \times 10$, were included in the experimental design in order to evaluate the effects of non-ideal boundary conditions, or pattern size effects. The selection of the specific geometrical design parameter values was irrelevant for the purpose of this study and therefore was arbitrarily determined. In addition, for all the designs, the thickness in the $z$ direction was arbitrarily set as $10 \mathrm{~mm}$. Additional information regarding the effects of these geometrical design parameters on the mechanical properties of the structures can be found in Section 3.2 .

Table 5.1 Details of the design parameters of each structure

\begin{tabular}{ccccccc}
\hline \multirow{3}{*}{ Structure } & Opening & Wall & Wall & Wall & Pattern size & Relative \\
& angle & length & length & thickness & $N$ & density \\
& $\theta\left(^{\circ}\right)$ & $L(\mathrm{~mm})$ & $H(\mathrm{~mm})$ & $t(\mathrm{~mm})$ & & $\rho$ \\
\hline
\end{tabular}




\begin{tabular}{lcccccc}
\hline Auxetic & 60 & 5.77 & 3.95 & 1 & $\begin{array}{c}5 \times 5, \\
8 \times 8,10 \times 10\end{array}$ & 0.362 \\
& & & & & $5 \times 5$, & \\
Diamond & 90 & 7.07 & - & 1 & $8 \times 8,10 \times 10$ & 0.265 \\
& & & & & $5 \times 5$, & \\
Triangular & 90 & 7.07 & - & 1 & $8 \times 8,10 \times 10$ & 0.411 \\
& & & & & & \\
\hline
\end{tabular}

For cellular structures with defect-free isotropic materials, due to the symmetry of both structural topology (Fig. 5.1) and boundary conditions, multiple topologically identical walls would achieve critical stress levels simultaneously. However, considering that in the real world there always exists some level of local material property variability, therefore, it can be reasonably assumed that only one wall would achieve maximum principal stress level realistically.

To quantify the effects of local property variability on the characteristics of the structures, the tensile strength and Young's modulus of the cellular walls were assumed to exhibit stochastic variabilities, which were represented by normal distributions of $\left(\mu_{s}, \sigma_{s}\right)$ and $\left(\mu_{m}, \sigma_{m}\right)$, respectively, where $\mu$ and $\sigma$ represent the mean and standard deviation of the distribution function. In the analytical model, the tensile strength and Young's modulus of each individual walls were generated randomly following the distibution functions, which means that the mechanical properties throughout the cellular structures are heterogeneous. In this study, Ti6Al4V was arbitrarily selected as the baseline solid material for the analytical calculation, with the Young's modulus of $114 \mathrm{GPa}$ and tensile strength of $1.05 G P a$. Furthermore, the strength variability $\left(\sigma_{s}\right)$ and modulus variability $\left(\sigma_{m}\right)$ were set as three different levels $(2 \%, 5 \%$ and $10 \%$ of the baseline). Consequently, in order to adequately capture the characteristic structural mechanical properties, a Monte-Carlo 
simulation approach was adopted, in which a sufficiently large number of iterations were generated and analyzed for each evaluation. The iteration number was set to be 20 for all the designs.

5.3 Effect of material property variability on the mechanical properties of cellular structures

\subsubsection{Effect of material strength variability}

First, the study was carried out with only material strength variability $(\sigma s)$ with three different levels, while the material modulus variability $(\sigma m)$ remained 0 . As the material strength variability is not expected to affect the elastic characteristics of the structures, only the strengths of the structures were investigated. Fig. 5.2-5.4 show the strain-stress curves for the three types of cellular structures of different unit cell numbers and different wall strength variabilities from the modeling results. For each design, 20 calculations were carried out at each of the strength variability levels, in order to account for the stochastic material property variability effects. To provide further baseline reference, the perfect structures without wall variabilities were also analyzed. In Fig. 5.2-5.4, the xaxis and y-axis of each curve indicate the strain and stress respectively, and different colors indicate different calculation cases. Each of the failure step is indicated by the abrupt reduction of stress levels in the strain-stress curves, which is a result of the brittle material behavior assumed by the models. 
(a) $\sigma_{\mathrm{s}}=\mathbf{0}, \sigma_{\mathrm{m}}=\mathbf{0}$

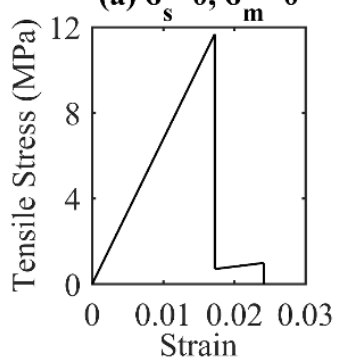

(e) $\sigma_{\mathrm{s}}=0, \sigma_{\mathrm{m}}=0$

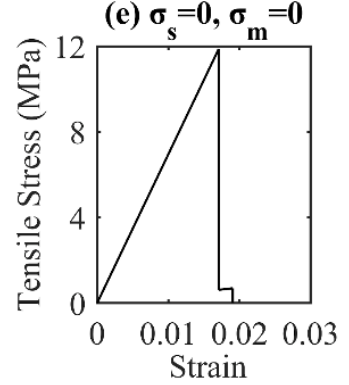

(i) $\sigma_{\mathrm{s}}=0, \sigma_{\mathrm{m}}=0$

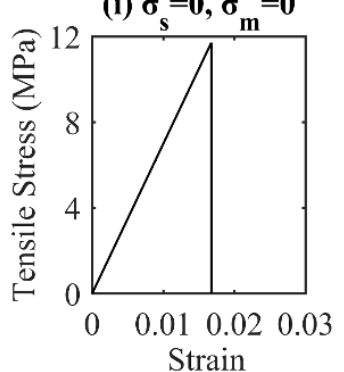

(b) $\sigma_{\mathrm{s}}=0.02, \sigma_{\mathrm{m}}=0$

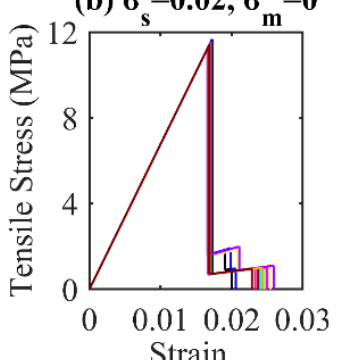

(f) $\sigma_{\mathrm{s}}=\mathbf{0 . 0 2}, \sigma_{\mathrm{m}}=\mathbf{0}$

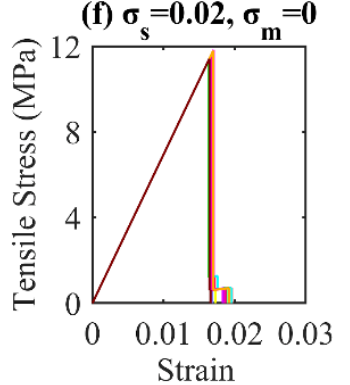

(j) $\sigma_{\mathrm{s}}=0.02, \sigma_{\mathrm{m}}=0$

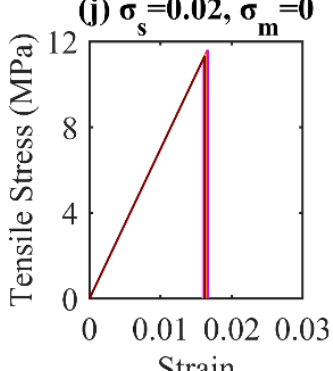

(c) $\sigma_{\mathrm{s}}=0.05, \sigma_{\mathrm{m}}=0$

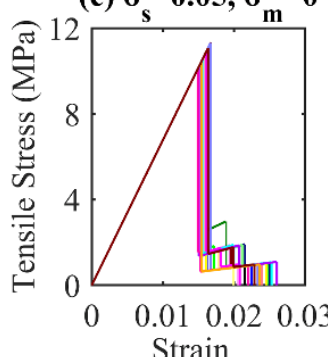

(g) $\sigma=0.05, \sigma_{\mathrm{m}}=0$

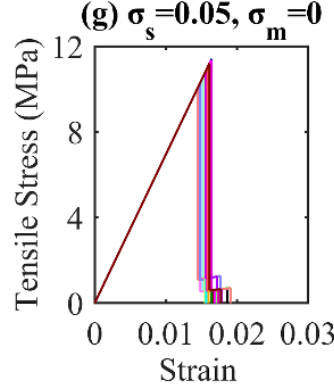

(k) $\sigma_{\mathrm{s}}=0.05, \sigma_{\mathrm{m}}=0$

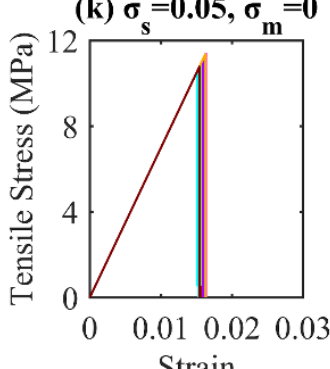

(d) $\sigma_{\mathrm{s}}=0.10, \sigma_{\mathrm{m}}=0$

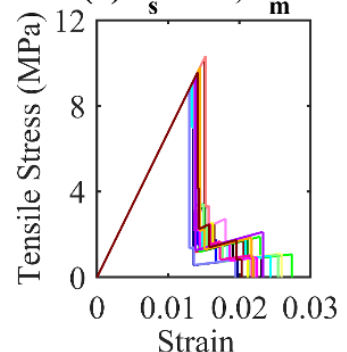

(h) $\sigma_{\mathrm{s}}=0.10, \sigma_{\mathrm{m}}=0$
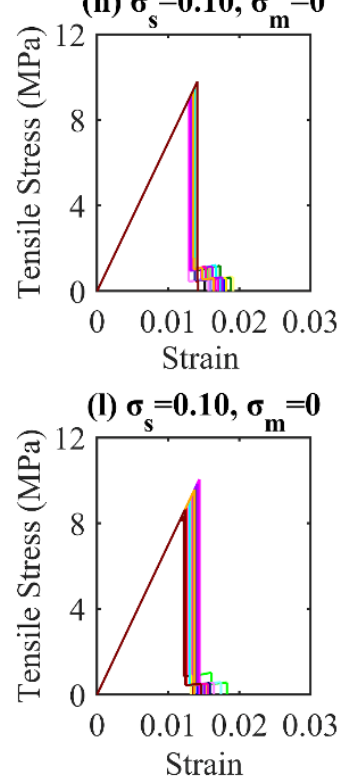

Fig. 5. 2 Strain-stress curves of auxetic structures with different unit cell numbers and wall strength variability:(a,b,c,d) Auxetic 5×5; (e,f,g,h)Auxetic 8×8; (i,j,k,l)Auxetic 10×10

Fig. 5.2 shows the strain-stress curves of the auxetic structures. These structures exhibit obvious saw tooth-like stress-strain patterns with multiple stress peaks during the tensile failure process, with critical stress levels gradually decrease. From Fig. 5.2, it is shown that when the wall strength variability decreases, the distribution of the strain-stress curves become more consistent and concentrated. In addition, the number of stress peaks also reduces as the standard deviation decreases, which indicated that the structures tend to exhibit more catastrophic fracture (i.e. fewer fracture steps) during the failure process. It is also found that when the unit cell numbers increase, the strain-stress curves also exhibit 
more consistent characteristics, and the structures exhibit relatively more catastrophic failure characteristics.
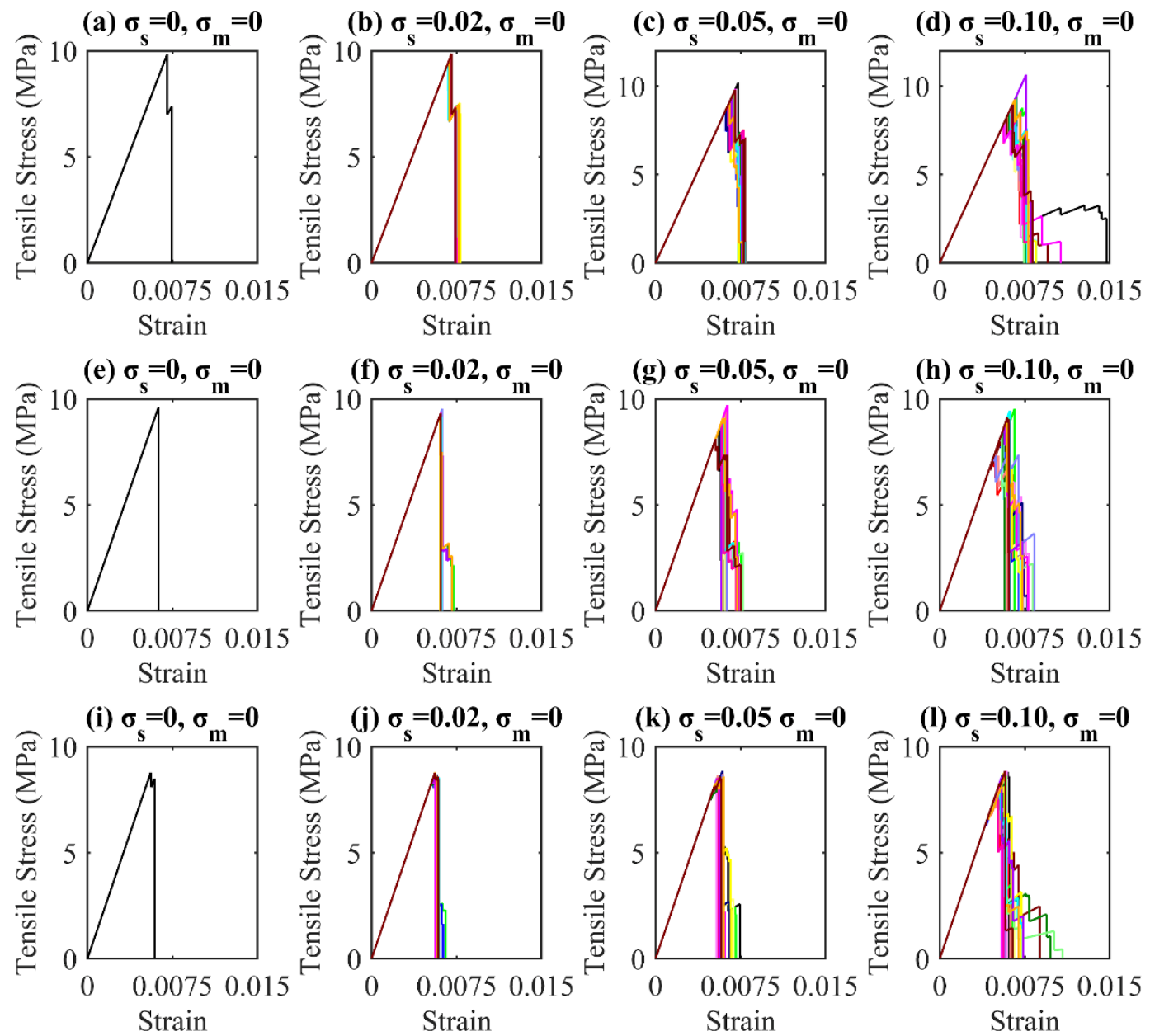

Fig. 5. 3 Strain-stress curves of diamond structures with different unit cell numbers and wall strength variability:(a,b,c,d) Diamond 5×5; (e,f,g,h) Diamond 8×8; (i,j,k,l) Diamond $10 \times 10$

Fig. 5.3 shows the strain-stress curves for the diamond structure. The diamond structures also exhibit increasingly consistent strain-stress characteristic and more catastrophic failure with higher unit cell numbers or smaller wall strength variability. On the other hand, there exists a considerable difference in the fracture steps between the diamond and the auxetic structures. The diamond structures tend to exhibit more fracture 
steps during the failure process. The multi-step failure characteristics of both the auxetic and diamond structures are further discussed in a later section.
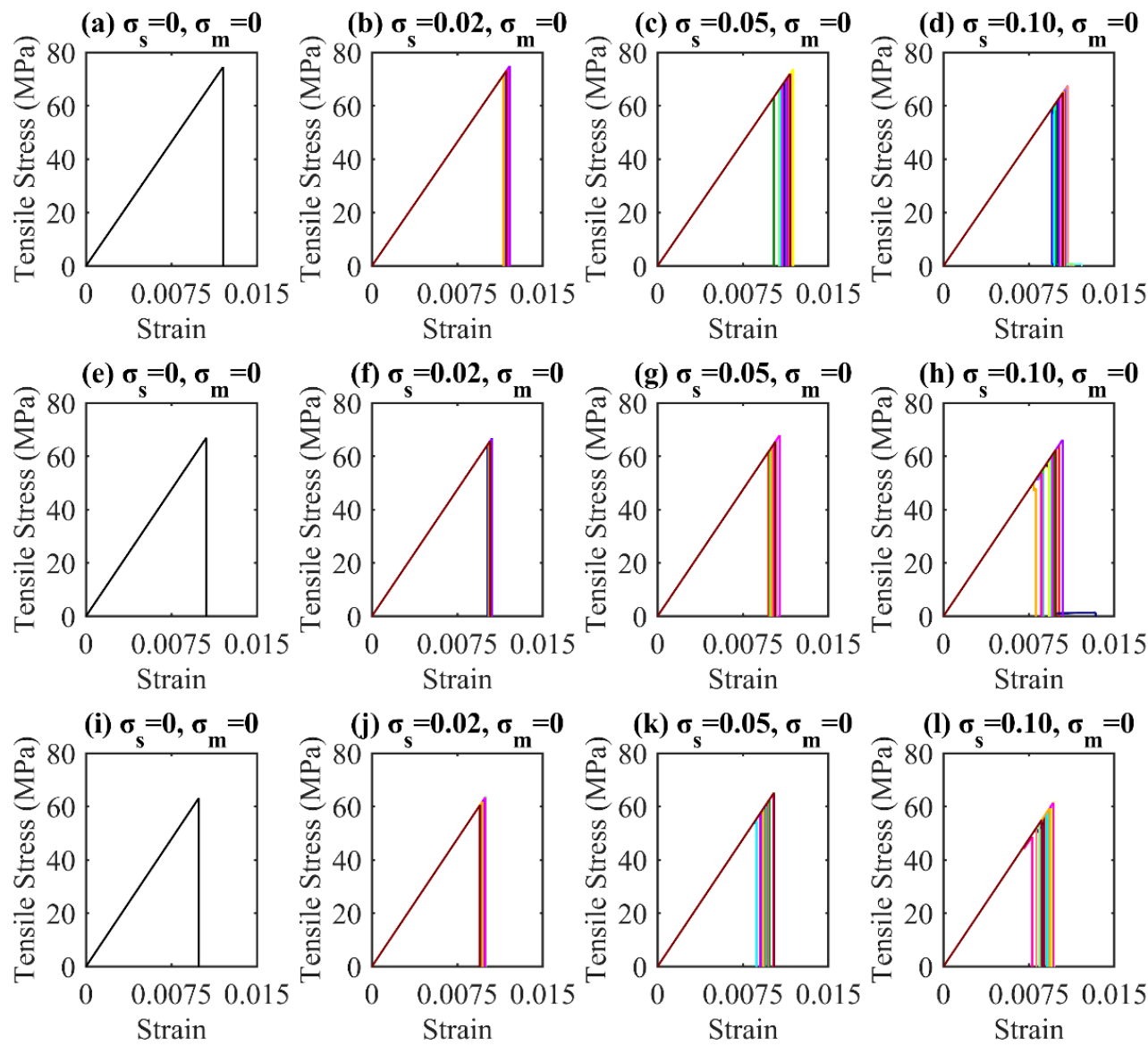

Fig. 5. 4 Strain-stress curves of triangular structures with different unit cell numbers and wall strength variability:(a,b,c,d) Triangular 5×5; (e,f,g,h) Triangular 8×8; (i,j,k,l) Triangular $10 \times 10$

Fig. 5.4 shows the strain-stress curves for the triangular structures. All the triangular structures exhibit only one stress peaks, which indicates a strictly catastrophic failure characteristic. While both the failure strength and the elongation at failure exhibit dependency on cell wall strength variability, they appear to be less affected by unit cell numbers. 


\subsubsection{Effect of cell wall strength variability on structural mechanical properties}

Fig. 5.5 shows the 20 calculated results of tensile strength for each of the three types of cellular structures of different pattern sizes and material strength variability levels. To provide further reference, the baseline structural strengths with perfect material were also calculated, which were indicated by solid red lines in Fig. 5.5. From the results, for all three types of cellular topologies, the increased material strength variability led to reduced tensile strength with the structures. Furthermore, with increasing material strength variability, the overall strength of the structures exhibits increasingly large variability, indicating that the structures not only become less strong but also become less consistent.

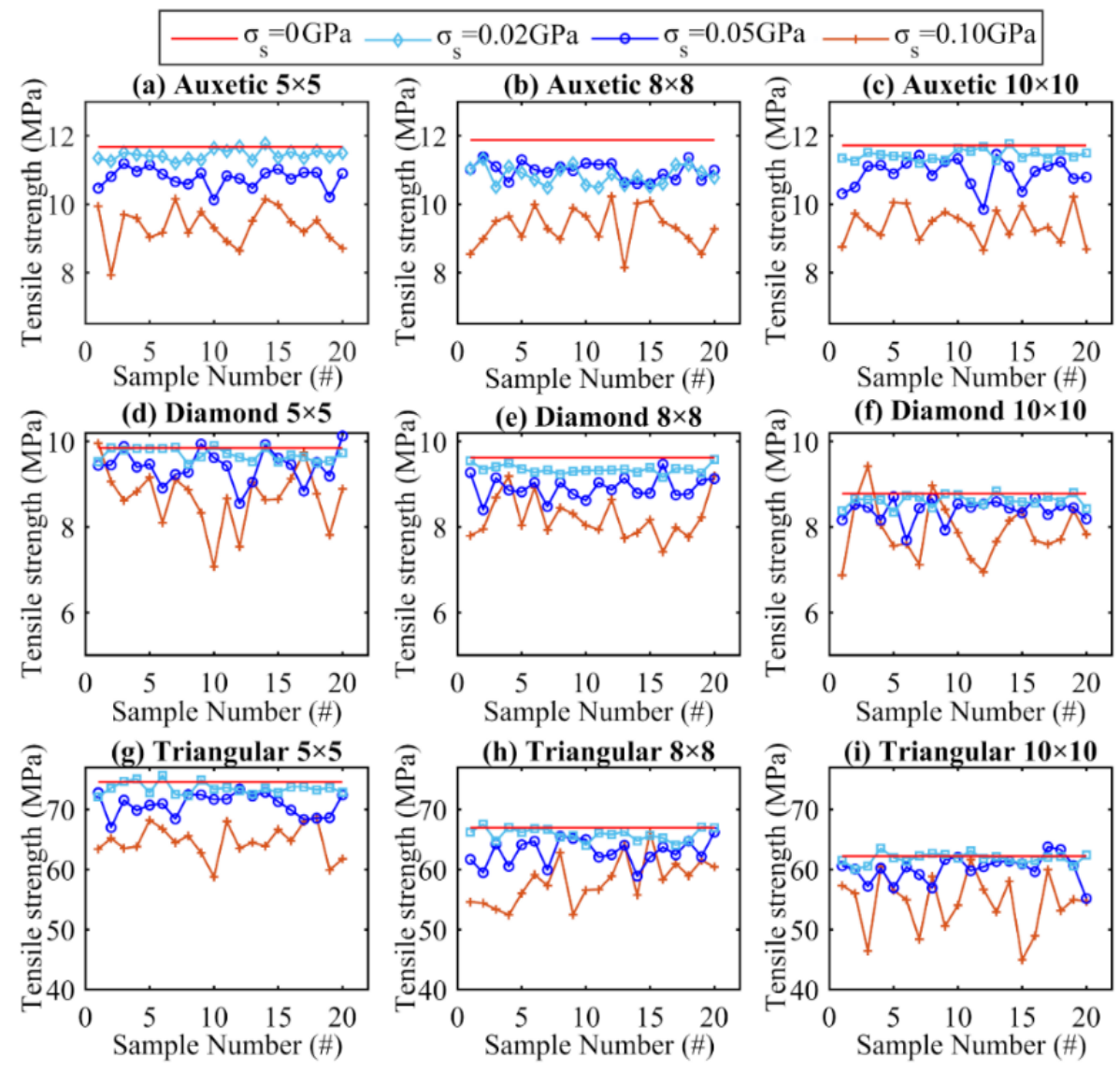

Fig. 5.5 Tensile strength calculation results for different designs with material strength variability 
Fig. 5.6 further summarizes the average and standard deviation values of the tensile strengths of each cellular design. For the auxetic structure, the pattern size appears to have little effect on its tensile strength regardless of the level of material strength variability. It has been previously suggested that auxetic structures generally exhibit minimum pattern size effects, and this study appears to confirm this conclusion. Furthermore, the results show that such characteristic of the auxetic structure is not affected by the local material strength imperfection. In comparison, for both the diamond and triangular structures, the strength of the structure reduces with increasing pattern sizes up to the maximum unit cell number of 10 investigated in this study.
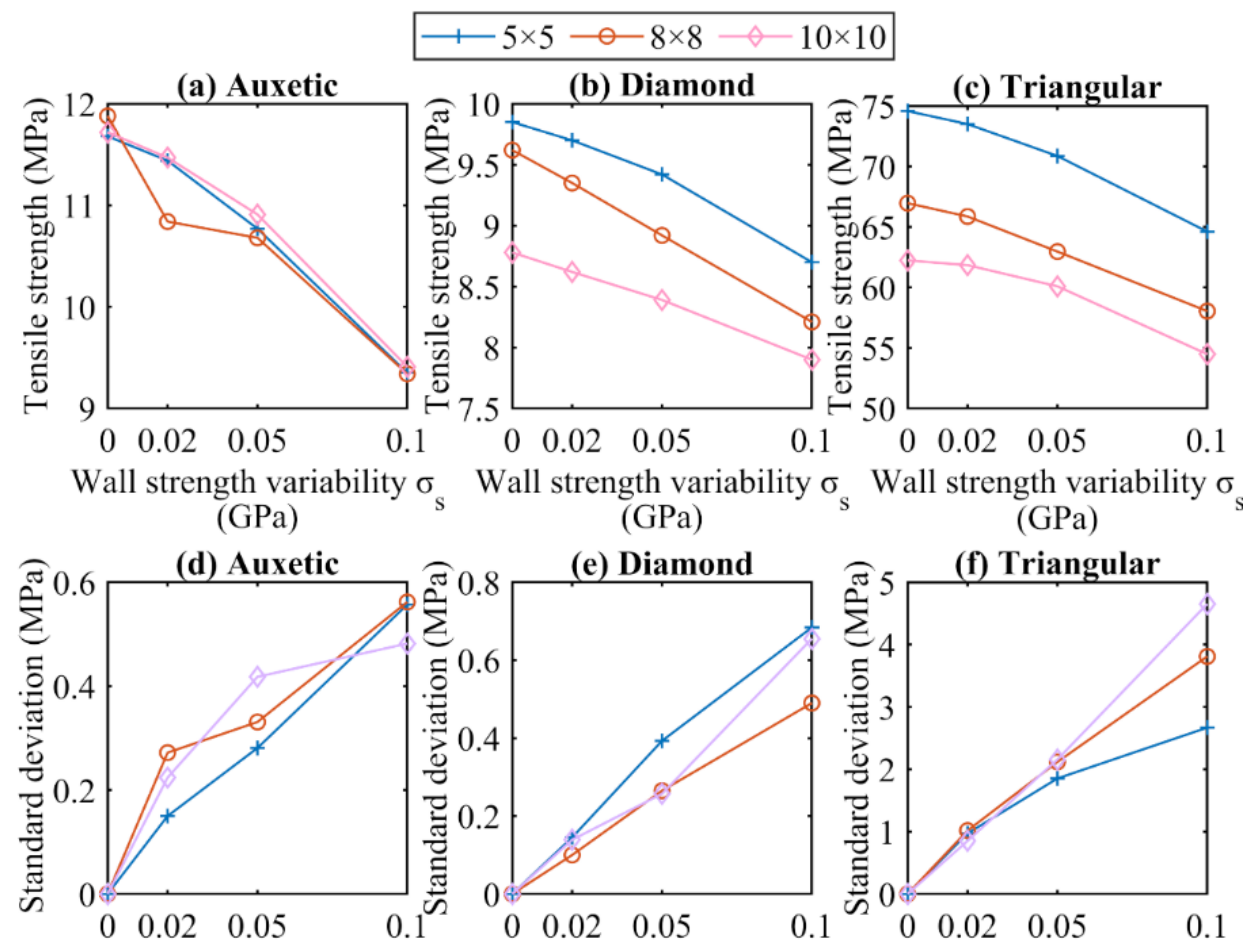

$\underset{(\mathrm{GPa})}{\text { Wall strength variability } \sigma_{\mathrm{s}}} \underset{(\mathrm{GPa})}{\text { Wall strength variability } \sigma_{\mathrm{s}}} \begin{gathered}\text { Wall strength variability } \sigma_{\mathrm{s}} \\ (\mathrm{GPa})\end{gathered}$

Fig. 5.6 The relationships between the material strength variability and average $(\mathrm{a}, \mathrm{b} \mathrm{c})$ / standard deviation $(\mathrm{d}, \mathrm{e}, \mathrm{f}))$ of the tensile strengths of the structures for different designs 
On the other hand, for all three types of cellular topologies, the relationships between the tensile strength of the structures and the level of material strength variability appear to be highly linear. Utilizing the baseline tensile strength of the structures (i.e. with perfect material), the tensile strength of the cellular could be expressed in the form of:

$$
\sigma_{\text {tensile }}=\sigma_{\text {Baseline }}-C_{1} * \sigma_{s}
$$

where the $\sigma_{\text {tensile }}$ is the average tensile strength of the structure, and the $\sigma_{\text {Baseline }}$ is the tensile strength of the baseline structure. Using linear regression fitting, the value of the slope factor $C_{1}$ is $22.59,8.77$ and 79.36 , with the correlations $\left(R^{2}\right)$ of $92.15 \%, 83.34 \%$ and $82.57 \%$ for auxetic, diamond and triangular structures, respectively. This indicates that among the three cellular structures studied in this paper, the stretching-dominated structure (triangular) exhibits significantly higher sensitivity to the material strength imperfection compared to the bending-dominated structures (auxetic and diamond). On the other hand, the tensile strength of the structures decreases by $4.3 \%, 2.1 \%$ and $1.2 \%$ for auxetic, diamond and triangular structures respectively, at a relatively low material strength variability level of $\sigma_{s}=0.02 \mathrm{GPa}(2 \%)$. When the material strength variability level increases to $\sigma_{S}=0.10 \mathrm{GPa}(10 \%)$, the tensile strength decreases by $20.3 \%, 12.1 \%$ and $13.0 \%$ for the three structures respectively. This indicates that the auxetic structure, while exhibiting many desirable characteristics, also appear to be most significantly impacted by the presence of material strength variability. This observation might be closely related to the small pattern size effect characteristic of the auxetic structures, which corresponds to smaller stress concentration effects induced by non-ideal boundary conditions (i.e. constraints and free surfaces), and consequently more pronounced "global" strength 
decrease as a result of the random occurrence of material property imperfection throughout the structures.

The standard deviations of the structural strengths obtained for each type of cellular design could be considered as approximations of the property variabilities of these structures. For all three types of cellular topologies, the strength variabilities of the structures appear to be only significantly influenced by the material strength variability but not the pattern size. Previous literature has shown that with varying structure pattern size the severity of boundary condition-induced stress localization changes. In addition, it was also observed that for all three types of topologies, the strengths of the structures generally exhibit less variability compared to the strength variability at the material level.

The multi-step failure characteristics of three structures can also be obtained through the strain-stress curves. Since the triangular structures show a catastrophic failure with just one stress peak, the stepwise failure strength and Young's modulus for two bending-dominated structures were investigated shown in Table 5.2 and Table 5.3 as they gradually fail. From the stepwise results of the auxetic structures shown in Table 5.2, the increase of the wall strength variability led to a decrease of the strength at step 1 and an increase of both the strength and modulus of the structures at step 2. This phenomenon is most noticeable for the small-size pattern $5 \times 5$ auxetic structure, in which the retaining failure strength and Young's modulus ratios (the retaining value divided by the step 1 value) increase from $8.5 \%$ to $28.7 \%$ and from $6.1 \%$ to $25.7 \%$, respectively, when the wall strength variability increases from 0 to $10 \%$.

Table 5. 2 Average tensile strength and Young's modulus of auxetic structures with different unit cell numbers and wall strength variabilities at each failure step 


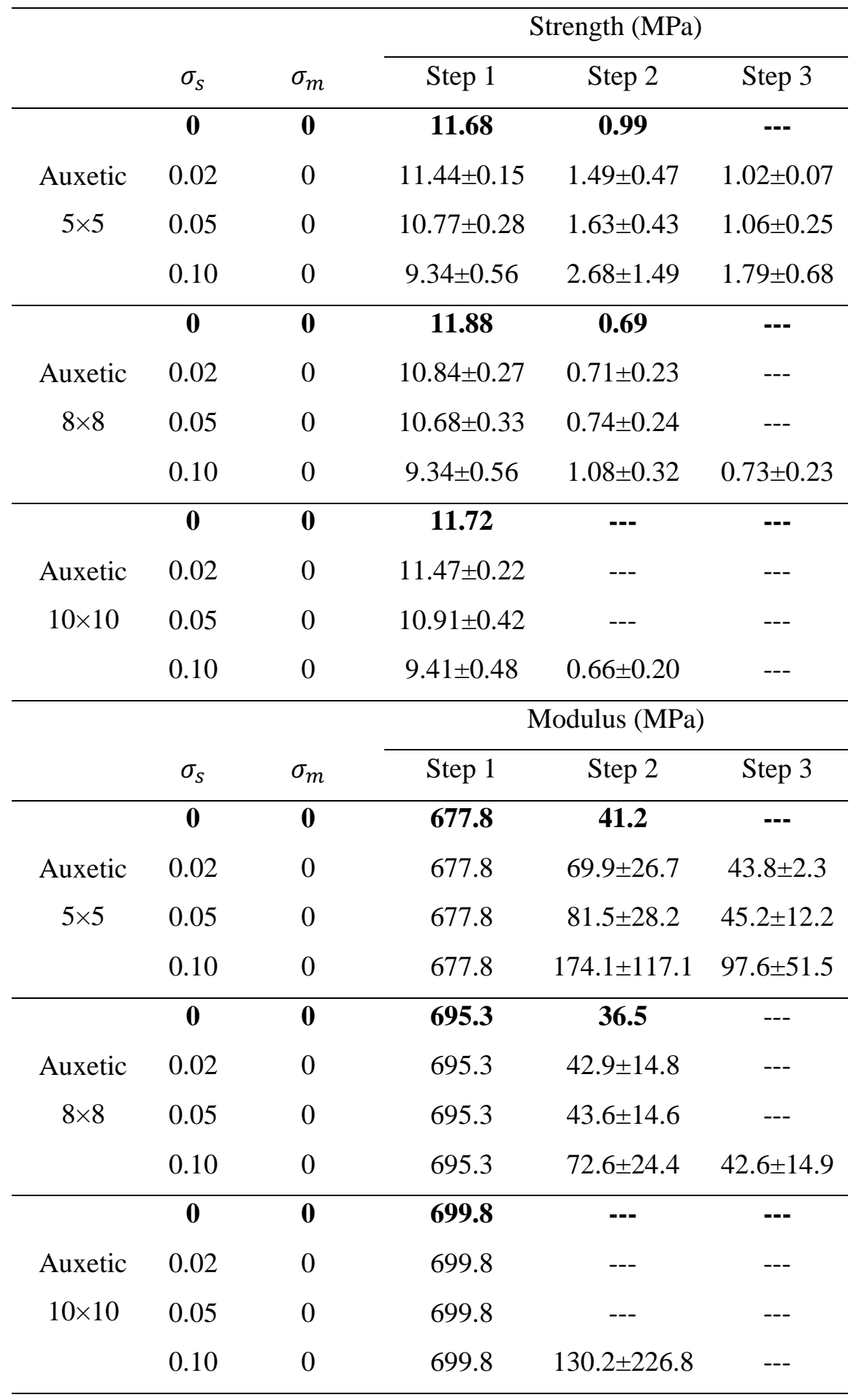

Table 5.3 shows the stepwise properties of the diamond structures. From the results, the increase of the wall strength variability generally corresponds to a decrease of stepwise failure strength. On the other hand, the effect of wall strength variability on the elastic 
modulus of the structure is less significant and less consistent. In addition, the wall strength variability also has a significant effect on the failure steps, with increased wall strength variability corresponds to more failure steps.

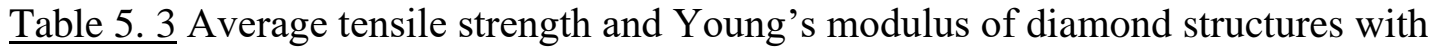
different unit cell numbers and wall strength variabilities at each failure step

\begin{tabular}{|c|c|c|c|c|c|c|c|}
\hline & \multirow[b]{2}{*}{$\sigma_{s}$} & \multirow[b]{2}{*}{$\sigma_{m}$} & \multicolumn{5}{|c|}{ Strength $(\mathrm{MPa})$} \\
\hline & & & Step 1 & Step 2 & Step 3 & Step 4 & Step 5 \\
\hline & $\mathbf{0}$ & $\mathbf{0}$ & 9.85 & 7.39 & --- & --- & --- \\
\hline Diamond & 0.02 & 0 & $9.67 \pm 0.14$ & $7.44 \pm 0.29$ & $5.18 \pm 2.44$ & --- & --- \\
\hline \multirow[t]{3}{*}{$5 \times 5$} & 0.05 & 0 & $9.42 \pm 0.39$ & $7.71 \pm 0.70$ & $6.43 \pm 1.45$ & $5.48 \pm 1.21$ & --- \\
\hline & 0.10 & 0 & $8.7 \pm 0.69$ & $7.64 \pm 0.84$ & $6.52 \pm 1.26$ & $5.47 \pm 1.81$ & $3.73 \pm 1.49$ \\
\hline & $\mathbf{0}$ & $\mathbf{0}$ & 9.62 & --- & --- & --- & --- \\
\hline Diamond & 0.02 & 0 & $9.35 \pm 0.10$ & --- & --- & --- & --- \\
\hline \multirow[t]{3}{*}{$8 \times 8$} & 0.05 & 0 & $8.92 \pm 0.26$ & $8.51 \pm 0.78$ & $6.21 \pm 1.71$ & --- & --- \\
\hline & 0.10 & 0 & $8.21 \pm 0.49$ & $7.70 \pm 1.22$ & $6.87 \pm 1.31$ & $5.76 \pm 1.41$ & $4.46 \pm 1.53$ \\
\hline & $\mathbf{0}$ & $\mathbf{0}$ & 8.78 & 8.48 & 8.47 & --- & --- \\
\hline Diamond & 0.02 & 0 & $8.62 \pm 0.14$ & $8.54 \pm 0.11$ & $8.49 \pm 0.09$ & $8.41 \pm 0.10$ & $8.35 \pm 0.06$ \\
\hline \multirow[t]{5}{*}{$10 \times 10$} & 0.05 & 0 & $8.39 \pm 0.26$ & $8.47 \pm 0.20$ & $7.78 \pm 1.59$ & $6.72 \pm 2.15$ & $4.80 \pm 2.53$ \\
\hline & 0.10 & 0 & $8.11 \pm 0.59$ & $7.91 \pm 0.63$ & $6.97 \pm 2.10$ & $5.96 \pm 2.70$ & $4.84 \pm 2.07$ \\
\hline & & & \multicolumn{5}{|c|}{ Modulus (MPa) } \\
\hline & $\sigma_{s}$ & $\sigma_{m}$ & Step 1 & Step 2 & Step 3 & Step 4 & Step 5 \\
\hline & $\mathbf{0}$ & $\mathbf{0}$ & 1398.9 & 992.6 & --- & --- & --- \\
\hline Diamond & 0.02 & 0 & $1398.9 \pm 0$ & $1008.6 \pm 67.1$ & $688.2 \pm 321.1$ & --- & --- \\
\hline \multirow[t]{3}{*}{$5 \times 5$} & 0.05 & 0 & $1398.9 \pm 0$ & $1108.7 \pm 122.2$ & $872.4 \pm 197.0$ & $736.8 \pm 161.1$ & --- \\
\hline & 0.10 & 0 & $1398.9 \pm 0$ & $1103.7 \pm 143.1$ & $891.3 \pm 176.2$ & $714.8 \pm 233.1$ & $482.9 \pm 190.7$ \\
\hline & $\mathbf{0}$ & $\mathbf{0}$ & 1532.8 & --- & --- & $\begin{array}{ll}-- \\
\end{array}$ & --- \\
\hline Diamond & 0.02 & 0 & $1532.8 \pm 0$ & --- & --- & --- & --- \\
\hline \multirow[t]{2}{*}{$8 \times 8$} & 0.05 & 0 & $1532.8 \pm 0$ & $1418.9 \pm 112.7$ & $1021.5 \pm 305.9$ & --- & --- \\
\hline & 0.10 & 0 & $1532.8 \pm 0$ & $1361.4 \pm 215.3$ & $1171.5 \pm 219.9$ & $954.5 \pm 246.1$ & $687.7 \pm 292.7$ \\
\hline Diamond & $\mathbf{0}$ & $\mathbf{0}$ & 1573.7 & 1503.6 & 1434.8 & 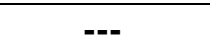 & --- \\
\hline
\end{tabular}




$\begin{array}{cccccccc}10 \times 10 & 0.02 & 0 & 1573.7 \pm 0 & 1538.7 \pm 0 & 1503.7 \pm 0.1 & 1469.2 \pm 0 & 1434.8 \pm 0 \\ & 0.05 & 0 & 1573.7 \pm 0 & 1538.7 \pm 0 & 1369.5 \pm 286.2 & 1156.6 \pm 390.1 & 791.0 \pm 438.4 \\ & 0.10 & 0 & 1573.7 \pm 0 & 1521.2 \pm 43.0 & 1243.3 \pm 396.3 & 1019.8 \pm 420.7 & 812.5 \pm 387.5\end{array}$

5.3.1.2 Effect of cell wall strength variability on failure response

The calculated results for effects of the unit cell numbers and cell wall strength variability on energy absorption are shown in Fig. 5.7. Again, the solid red lines in Fig. 5.7 indicate the baseline energy absorption of the cellular structures with no material property variability. The existence of the cell wall strength variability led to an obvious decrease of the energy absorption for the auxetic structure and triangular structure. For these two structures, larger cell wall strength variability generally corresponds to more significant decreases in the energy absorption of the cellular structures. On the other hand, for the diamond structure, the cell wall strength variability does not appear to have significant effect on the average energy absorption, although with increasing cell wall strength variability the energy absorption ability of the structure also appears to exhibit increased variability. On the other hand, the effect of the unit cell numbers on the energy absorption of different structures exhibits the opposite characteristic. For the auxetic structures, the effect of unit cell number appears insignificant, while for both the diamond and triangular structures, the average energy absorption capabilities of the structures decrease as the unit cell numbers increases. 

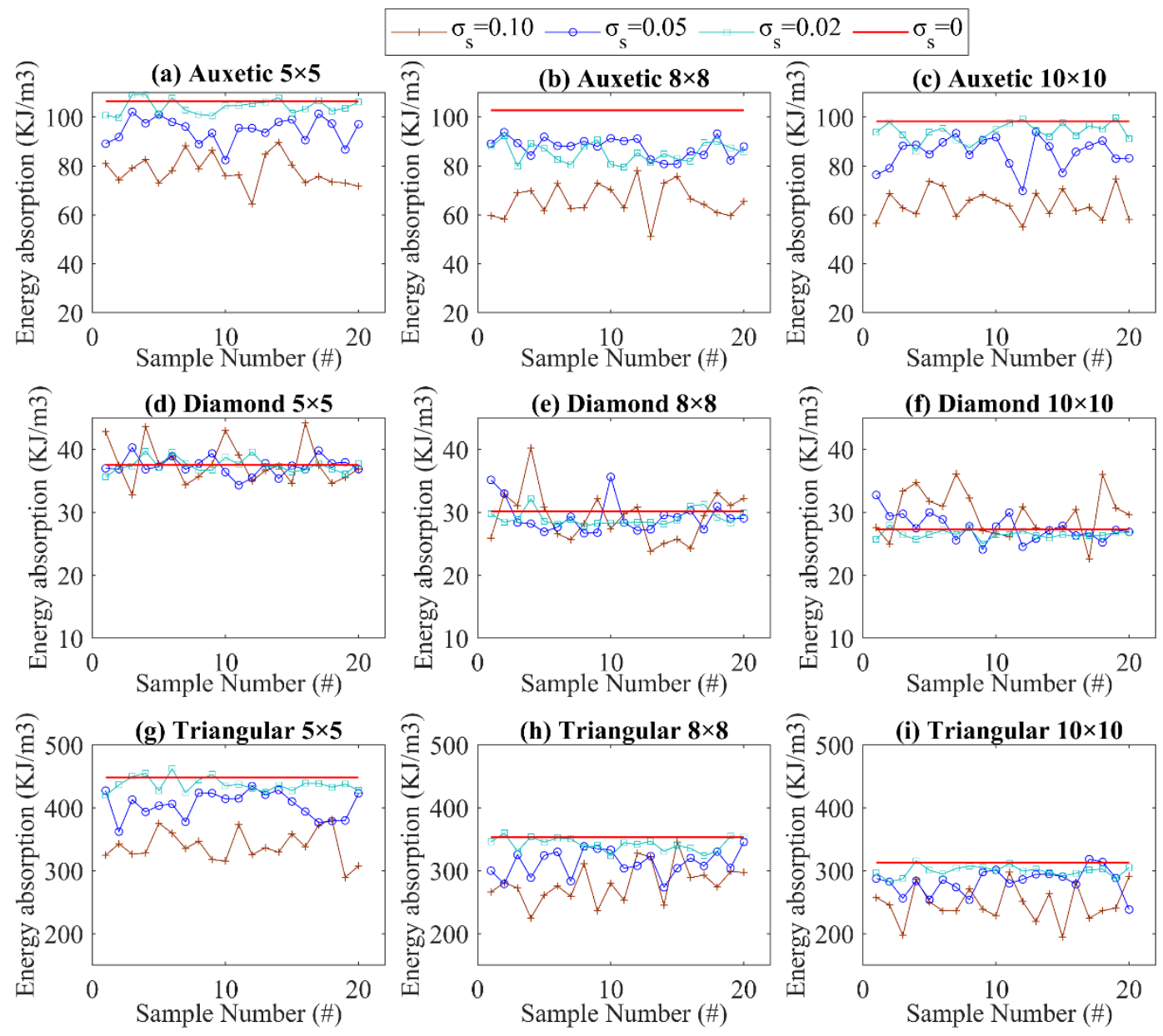

Fig. 5. 7 Energy absorption variability of different designs

Fig.5.8 further illustrates the average energy absorption and energy absorption variability from the 20 calculations for each design. For auxetic and triangular structures, as the wall strength variability increases, the average energy absorption decreases, and the energy absorption variability increases. On the other hand, for the diamond structures, the wall strength variability has little effect on the average energy absorption of the structure. 

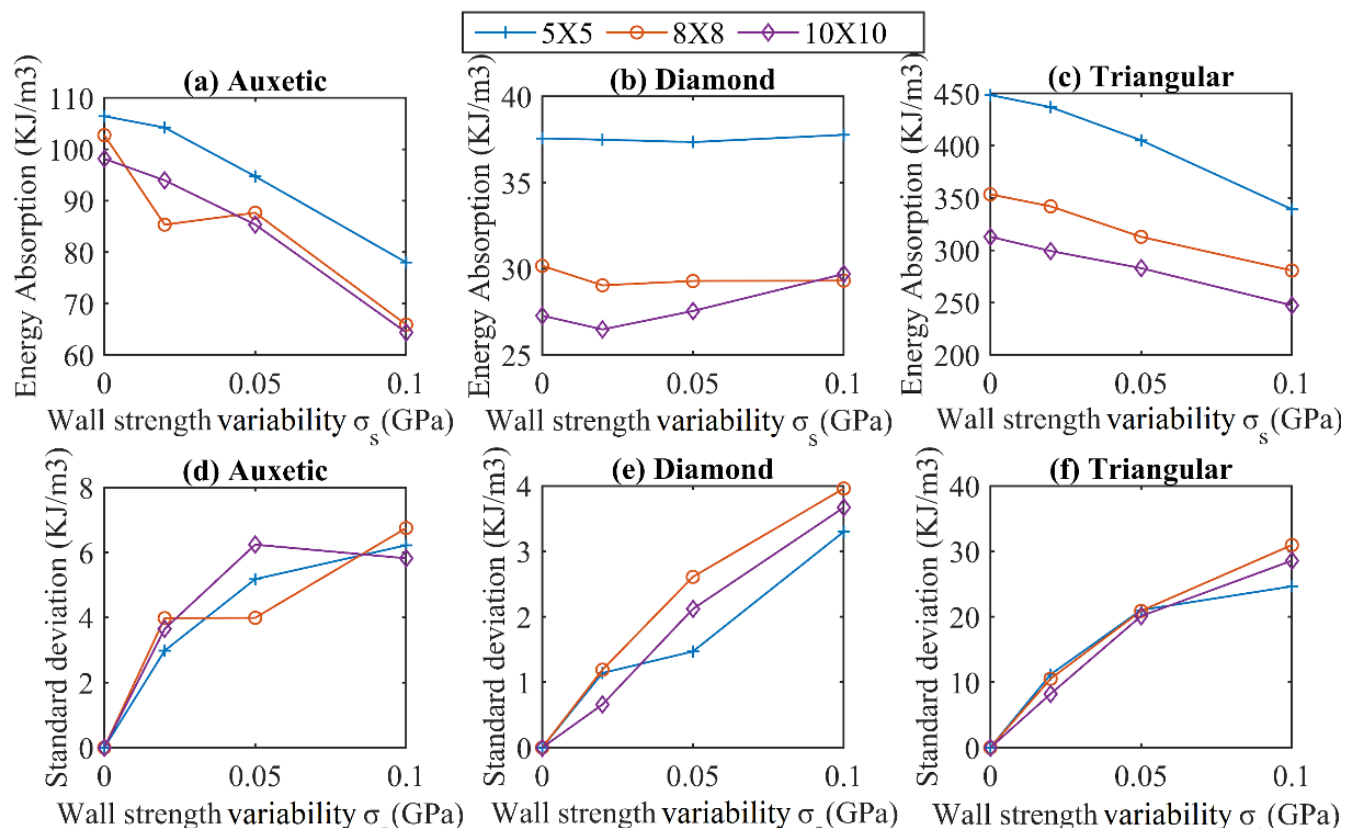

Wall strength variability $\sigma_{\mathrm{s}}(\mathrm{GPa})$ Wall strength variability $\sigma_{\mathrm{s}}(\mathrm{GPa})$

Wall strength variability $\sigma_{\mathrm{s}}(\mathrm{GPa})$

Fig. 5.8 The relationships between the wall strength variability levels and mean $(\mathrm{a}, \mathrm{b} \mathrm{c})$ / standard deviation $(\mathrm{d}, \mathrm{e}, \mathrm{f})$ of cellular energy absorption of different designs

Fig. 5.9-5.11 show the failure propagation patterns of the three types of cellular structures with different unit cell numbers and cell wall strength variability. It is noted that for visual clarify, the dimensions of these schematics do not match the actual designs. It should also be noted that due to the structural symmetry with the cellular designs, each specific failure pattern cases shown in Fig. 5.9-5.11 can be interpreted as representative to three other cases (i.e. under left-right and top-bottom mirror symmetries).

Fig. 5.9 shows the failure patterns of 2D auxetic structures under different material and pattern conditions. Fig. 5.9(a-c) each shows the failure patterns from 16 runs of 5x5 auxetic patterns of different material strength variability levels. Fig.5.9(d-f) and Fig.5.9(gi) show the same type of information of the $8 \times 8$ and $10 \times 10$ patterns, respectively. With small amount of material strength variability, the fracture of the auxetic structures typically initiates from near one of the corners, then propagates towards the center of the structure, 
before extending to the opposite side of the boundary. With increasing material strength variability, the fracture patterns exhibit increased tendency of "side-to-side" mode, with the fracture initiation occurrence transitioning away from the corners and towards the middle of the side boundary. The number of unit cells does not appear to introduce significant change of fracture pattern modes. This observation appears consistent to the other mechanical properties for auxetic structure previously discussed in this study.

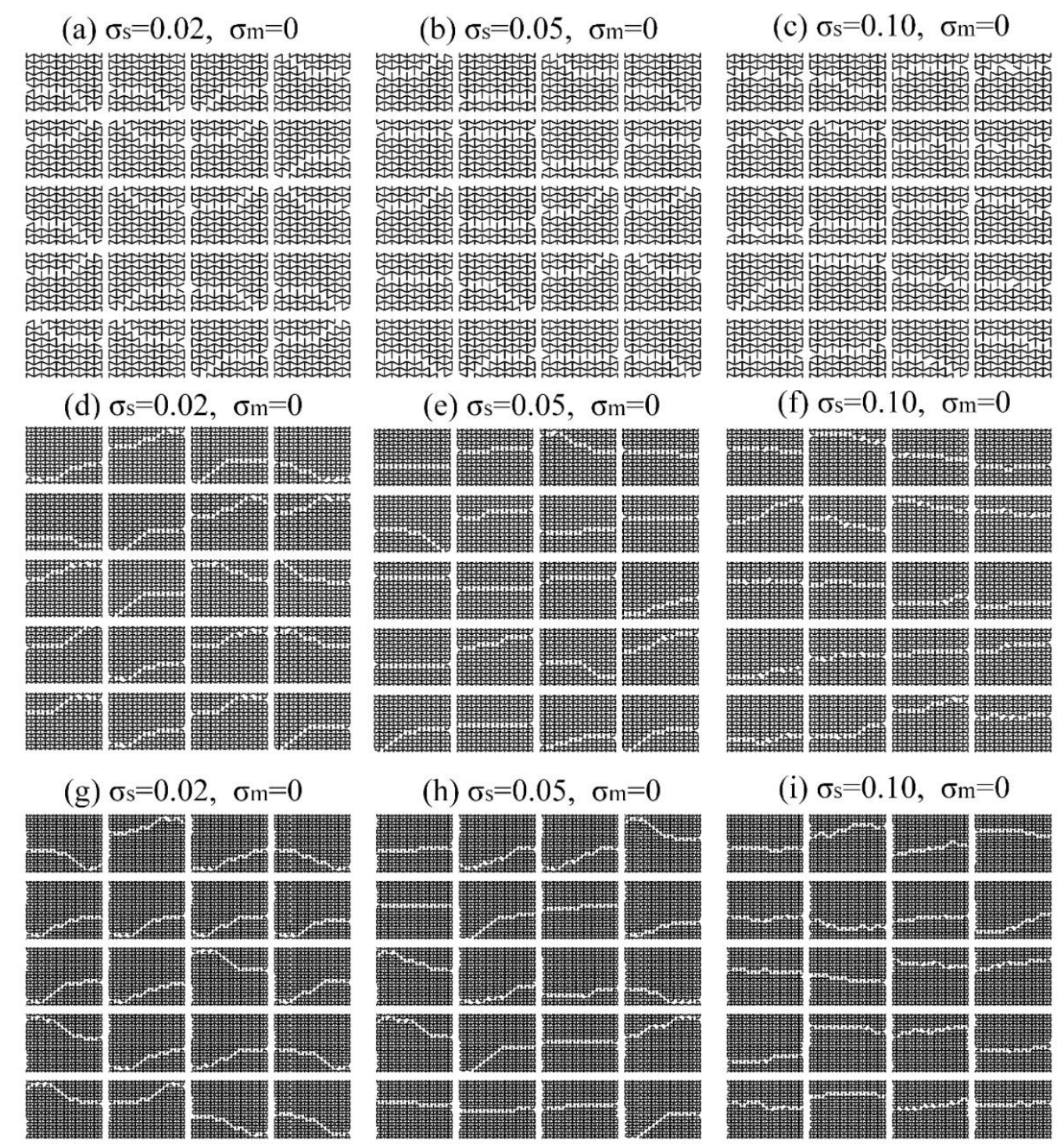

Fig. 5.9 The failure patterns of the auxetic structures with different unit cell numbers and wall strength variabilities 
(a) $\sigma_{\mathrm{s}}=0.02, \sigma_{\mathrm{m}}=0$

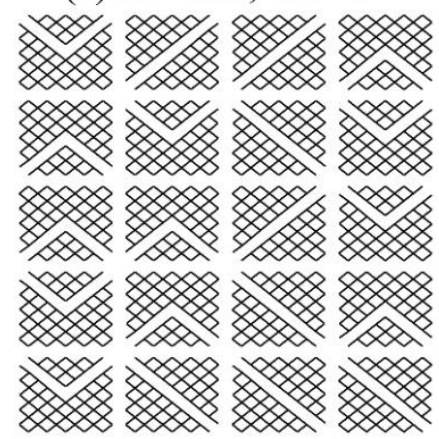

(d) $\sigma_{\mathrm{s}}=0.02, \sigma_{\mathrm{m}}=0$

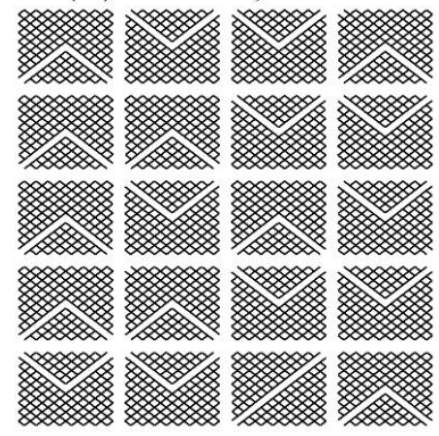

(g) $\sigma_{\mathrm{s}}=0.02, \sigma_{\mathrm{m}}=0$

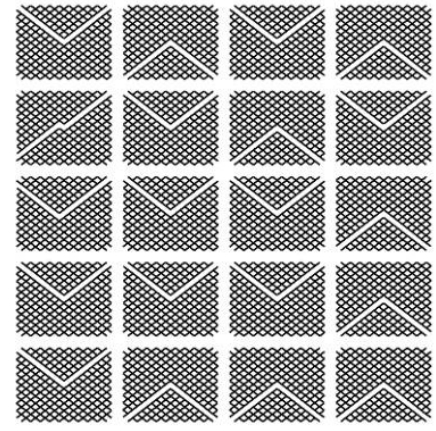

(b) $\sigma_{\mathrm{s}}=0.05, \sigma \mathrm{m}=0$

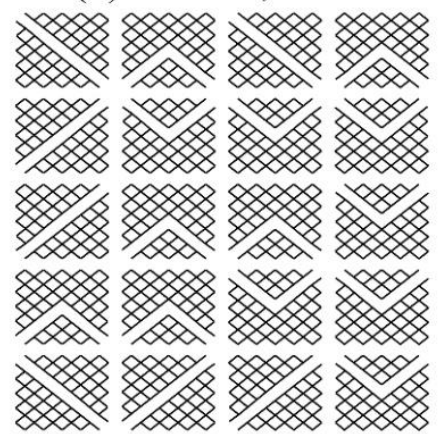

(e) $\sigma_{\mathrm{s}}=0.05, \sigma \mathrm{m}=0$

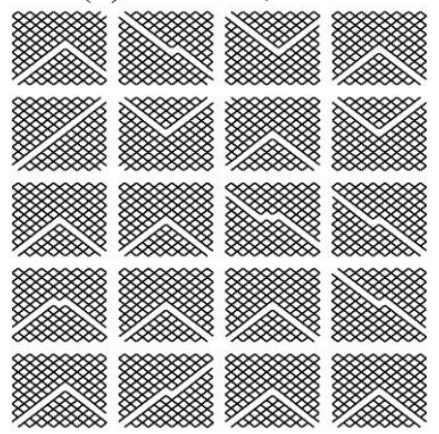

(h) $\sigma_{\mathrm{s}}=0.05, \sigma \mathrm{m}=0$

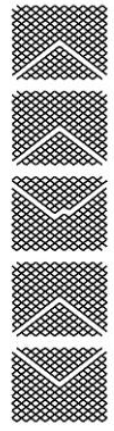

(c) $\sigma_{\mathrm{s}}=0.10, \sigma_{\mathrm{m}}=0$

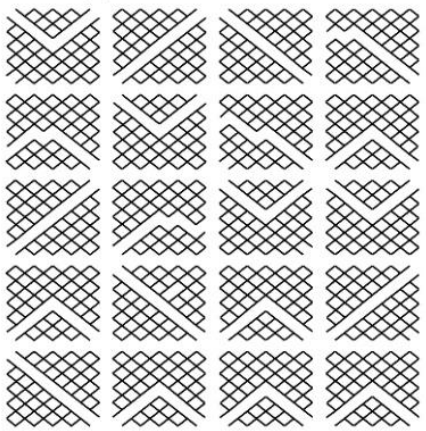

(f) $\sigma_{\mathrm{s}}=0.10, \sigma \mathrm{m}=0$

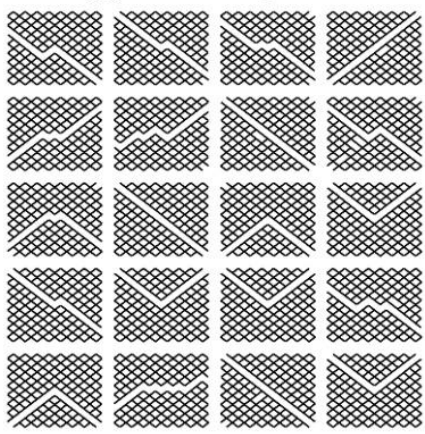

(i) $\sigma_{\mathrm{s}}=0.10, \sigma_{\mathrm{m}}=0$

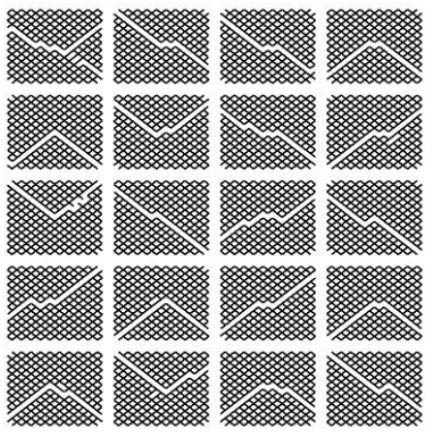

Fig. 5.10 The failure patterns of the diamond structures with different unit cell numbers and wall strength variabilities

Fig.5.10 shows the failure patterns of the 2D diamond structure. Fig. 5.10(a-c), Fig.5.10(d-f), and Fig.5.10(g-i) show the results from runs of $5 \times 5,8 \times 8$ and $10 \times 10$ patterns, respectively. In general, the diamond structures exhibit a highly consistent diagonal or Vshape fracture patterns that initiate from one of the corners of the structures. The wall strength variability appears to have more significant effect on the fracture pattern modes 
compared to the unit cell numbers. Under high wall strength variability $\left(\sigma_{s}=0.10\right)$, the diamond structures exhibit more fracture pattern irregularities even though the overall fracture patterns are still diagonal or V-shape.

Fig. 5.11 shows the failure patterns of the 2D triangular structure. Fig. 5.11(a-c), Fig.5.11(d-f) and Fig.5.11(g-i) show the results from runs of $5 \times 5,8 \times 8$ and $10 \times 10$ patterns, respectively. Overall, the fracture patterns of the triangular structure tend to concentrate towards the corner regions of the structures regardless of the wall strength variability and pattern size. With increasing wall strength variability, the fracture patterns exhibit increasing trend of irregularity, and on some occasions even deviate from the corner regions (Fig.5.11(c) and Fig.5.11(f)). On the other hand, with increasing number of unit cells, such fracture mode deviation becomes less significant. 
(a) $\sigma \mathrm{s}=0.02, \sigma \mathrm{m}=0$

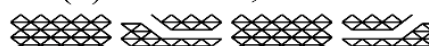

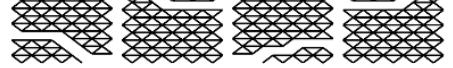

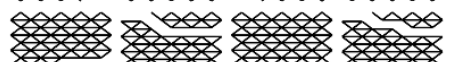
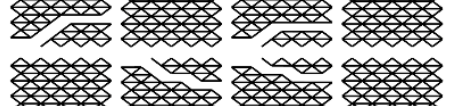

\& $\quad \forall \%$

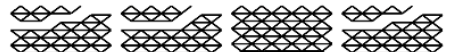

\% $\quad 4 \quad \%$

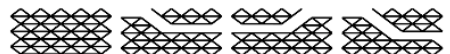

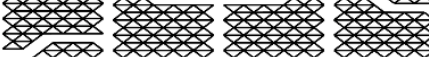

(d) $\sigma_{\mathrm{s}}=0.02, \sigma_{\mathrm{m}}=0$

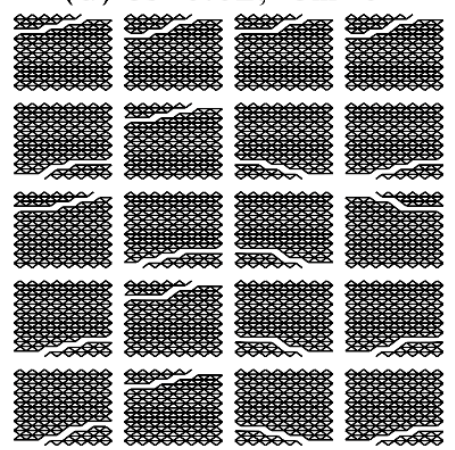

$(\mathrm{g}) \sigma_{\mathrm{s}}=0.02, \sigma_{\mathrm{m}}=0$

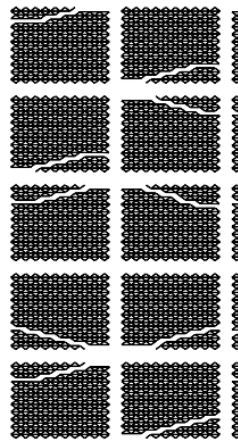

(b) $\sigma_{\mathrm{s}}=0.05, \sigma \mathrm{m}=0$

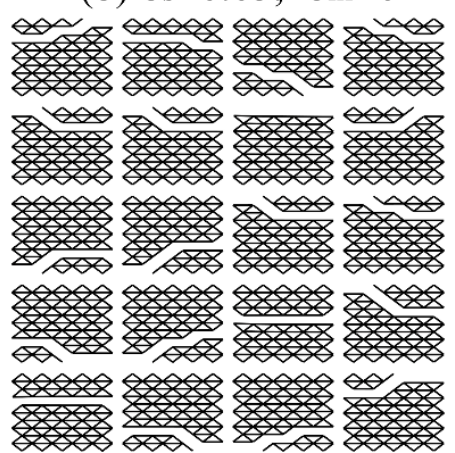

(e) $\sigma_{\mathrm{s}}=0.05, \sigma_{\mathrm{m}}=0$

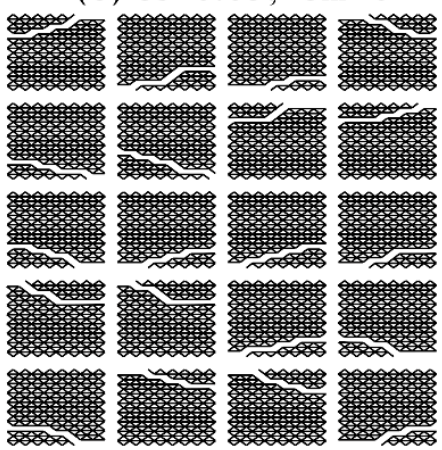

(h) $\sigma_{\mathrm{s}}=0.05, \sigma_{\mathrm{m}}=0$
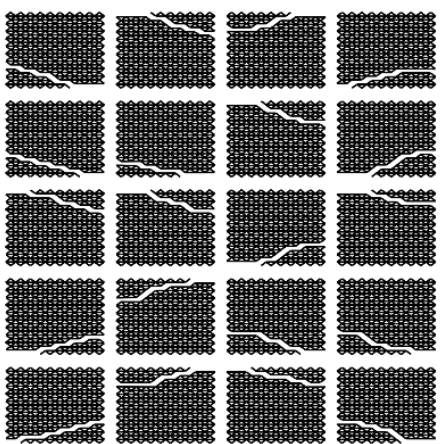

(c) $\sigma_{\mathrm{s}}=0.10, \sigma_{\mathrm{m}}=0$

\&

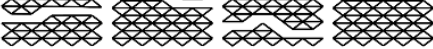
W $\quad 4 \quad$ L $\begin{array}{llll} & & \end{array}$ W

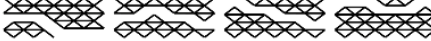

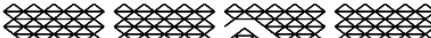
W \& $\quad 2 \quad 1$ $\begin{array}{lllll} & \end{array}$

(f) $\sigma_{\mathrm{s}}=0.10, \sigma \mathrm{m}=0$

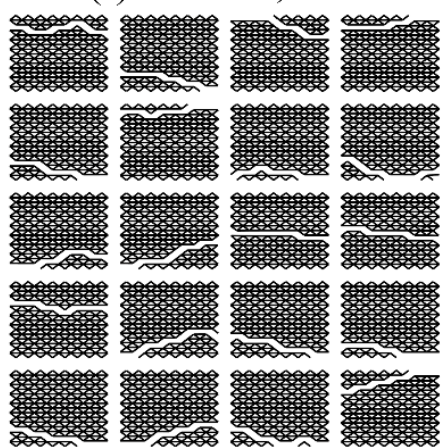

(i) $\sigma_{\mathrm{s}}=0.10, \sigma_{\mathrm{m}}=0$

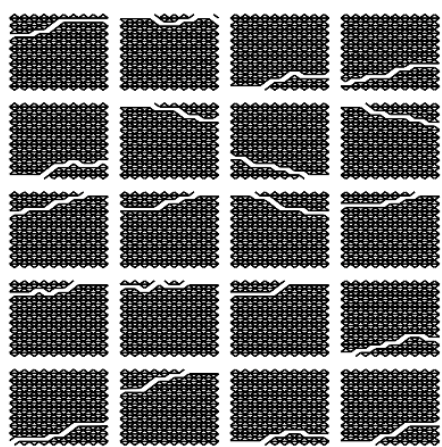

Fig. 5. 11 The failure patterns of the triangular structures with different unit cell numbers and wall strength variabilities

\subsubsection{Effect of material modulus variability}

In this section, the study was carried out with only material modulus variability $\left(\sigma_{m}\right)$ with three different levels, while the material strength variability $\left(\sigma_{s}\right)$ remained 0 . As the material modulus variability will affect both the elastic and strength characteristics of the structures, both of them were investigated here. Fig. 5.12-5.14 shows the strain-stress 
curves for the three types of cellular structures of different unit cell numbers and different wall modulus variabilities from the modeling results. For each design, 20 runs were analyzed, which are indicated by different colors. For each run, the tensile strength of the wall was kept constant, while randomly generated Young's modulus values were assigned to each cellular wall. Three different modulus variability levels $(2.28 \mathrm{GPa}, 5.70 \mathrm{GPa}$ and $11.4 \mathrm{GPa}$ ) were investigated, which corresponded to $2 \%, 5 \%$ and $10 \%$ of the Young's modulus of the perfect material. The results with perfect material were used to provide baseline reference.

Fig. 5.12 shows the strain-stress curves for the auxetic structures with wall modulus variability. The results show that the wall modulus variability does not have significant effect on the elastic modulus of the structures and slightly affect their initial failure strength. The structures tend to exhibit higher initial failure strength variability and more stress peaks with higher elastic modulus variability. In addition, the stress-strain characteristics of the structures also exhibit more consistency when the unit cell numbers increase. 

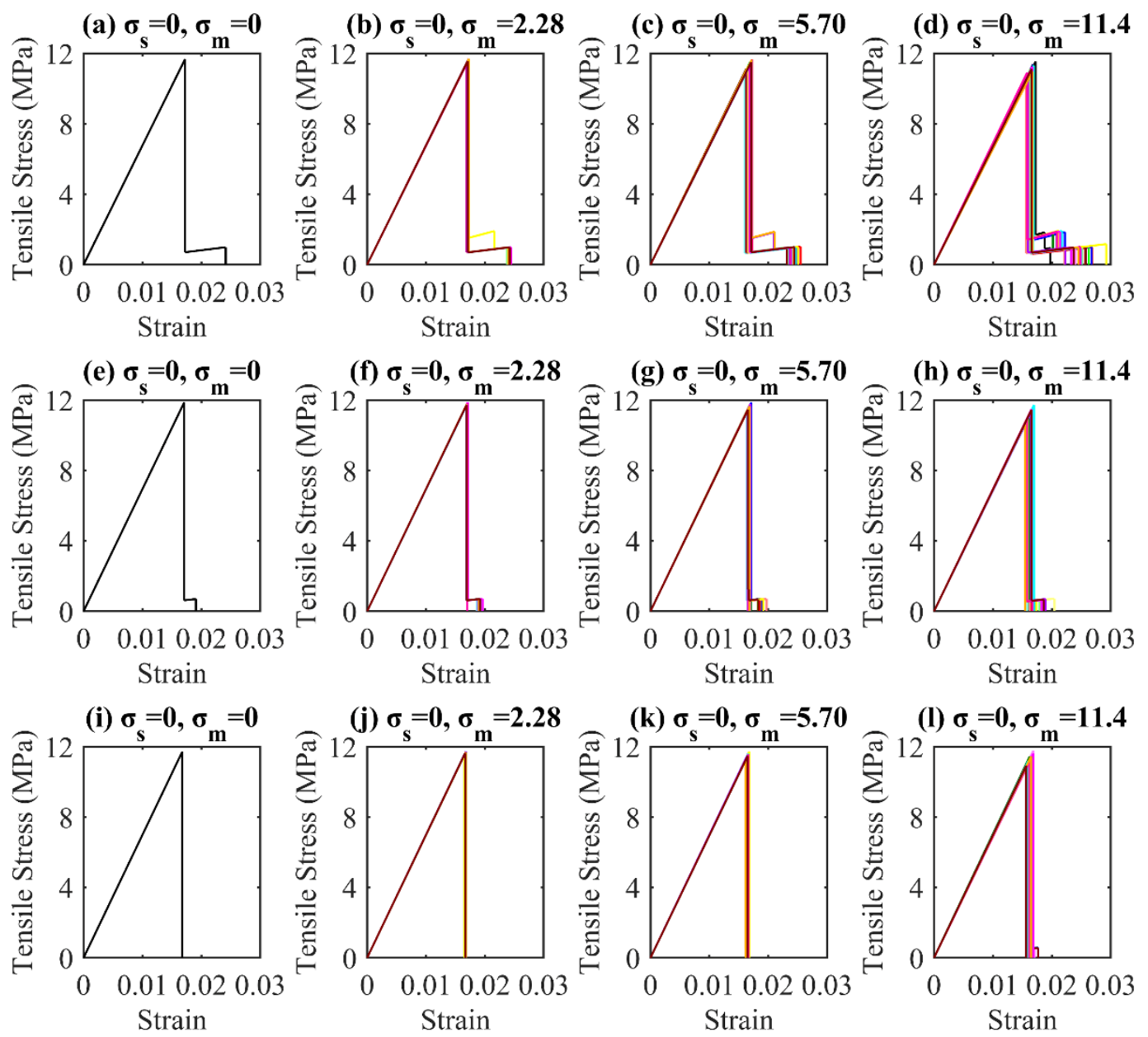

Fig. 5. 12 Strain-stress curves of auxetic structures with different unit cell numbers and wall modulus variabilities:(a,b,c,d) Auxetic 5×5; (e,f,g,h)Auxetic 8×8; (i,j,k,l)Auxetic $10 \times 10$

Fig. 5.13 shows the strain-stress curves for the diamond structures with wall modulus variability. In general, the wall modulus variability does not appear to have significant effect on the elastic modulus of the structures but slightly affects the initial failure strength levels. Higher modulus variability leads to higher initial failure strength variability as well as more stress peaks. These observations are consistent with those from the auxetic structures, indicating the possibility of common characteristics for bendingdominated designs. 

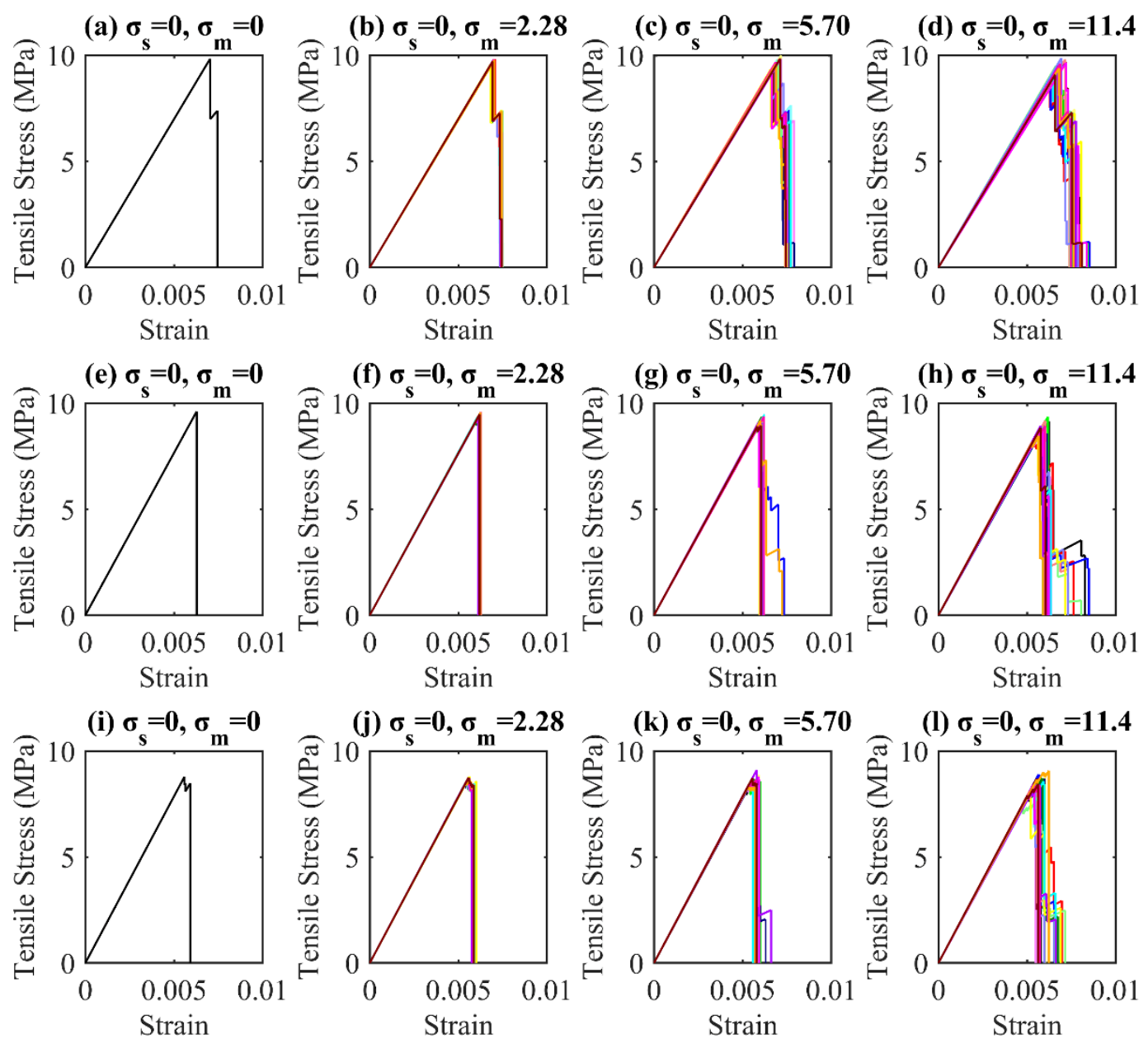

Fig. 5.13 Strain-stress curves of auxetic structures with different unit cell numbers and wall modulus variabilities:(a,b,c,d) Diamond 5×5; (e,f,g,h) Diamond 8×8; (i,j,k,l) Diamond $10 \times 10$

Fig. 5.14 shows the strain-stress curves for the triangular structures with wall modulus variability. While the wall modulus variability also introduced some initial failure strength variability with the triangular designs, all the structures exhibited only one stress peak. 

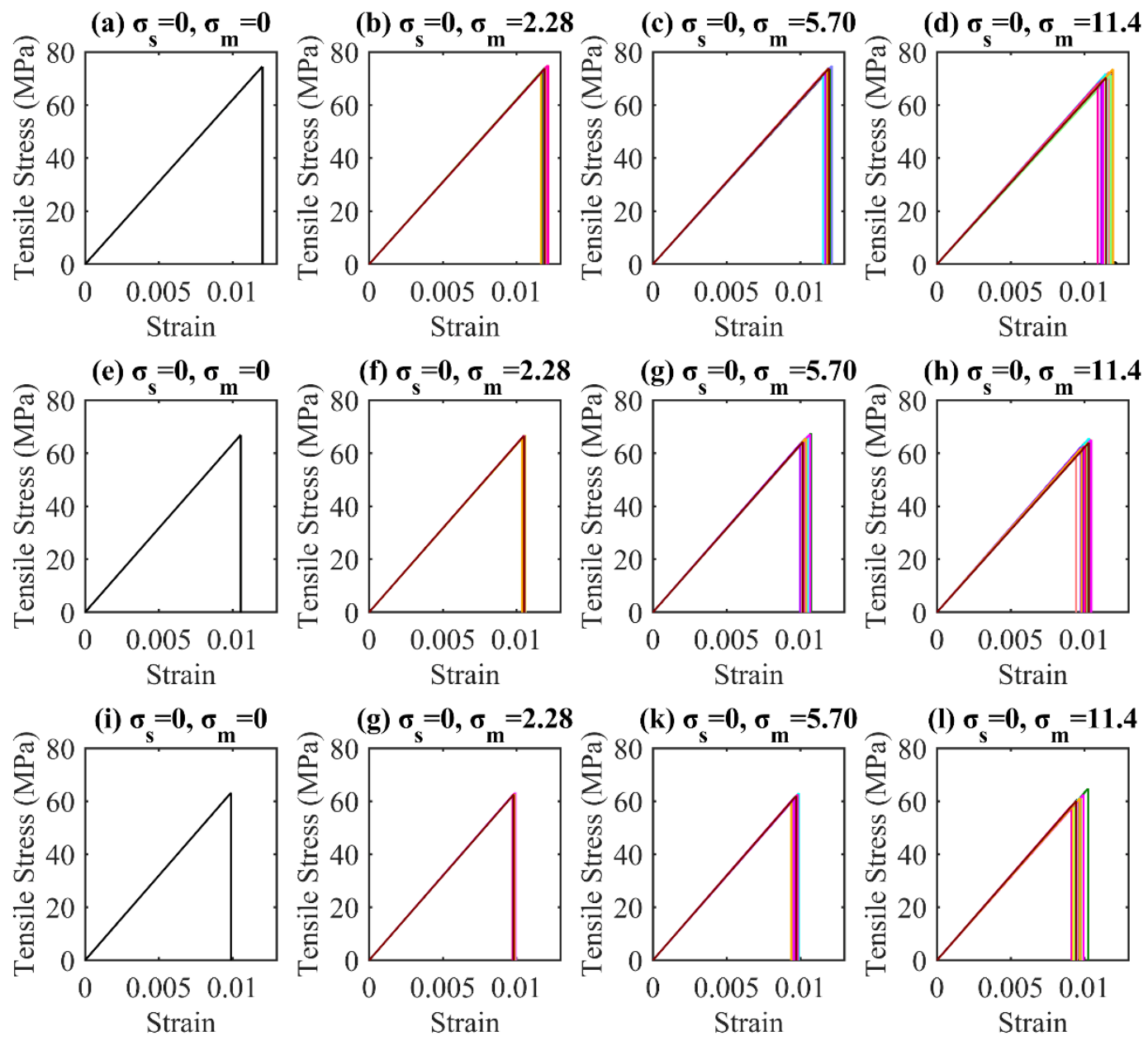

Fig. 5.14 Strain-stress curves of auxetic structures with different unit cell numbers and wall modulus variabilities:(a,b,c,d) Triangular 5×5; (e,f,g,h) Triangular $8 \times 8$; (i,j,k,l) Triangular $10 \times 10$

\subsubsection{Effect of cell wall modulus variability on structural mechanical properties}

Fig. 5.15 and Fig. 5.16 show the calculated results of structural tensile strength and elastic modulus for each of the three types of cellular structures of different pattern sizes and material elastic modulus variability levels. The baselines with perfect material $\left(\sigma_{m}=0\right)$ were also included as solid red lines. From Fig. 5.15, the results clearly show that the tensile strength of the cellular structures is also affected by the material elastic modulus variability. 
As material elastic modulus variability increases, the average tensile strengths of the cellular structures decrease, while their strength variability levels increase. It should be noted that in real-world scenarios there is rarely a case where only elastic modulus exhibits variability with a material. However, the observations still provide useful insights about the contributions of different types of material property variability to the overall variability of the cellular structures.

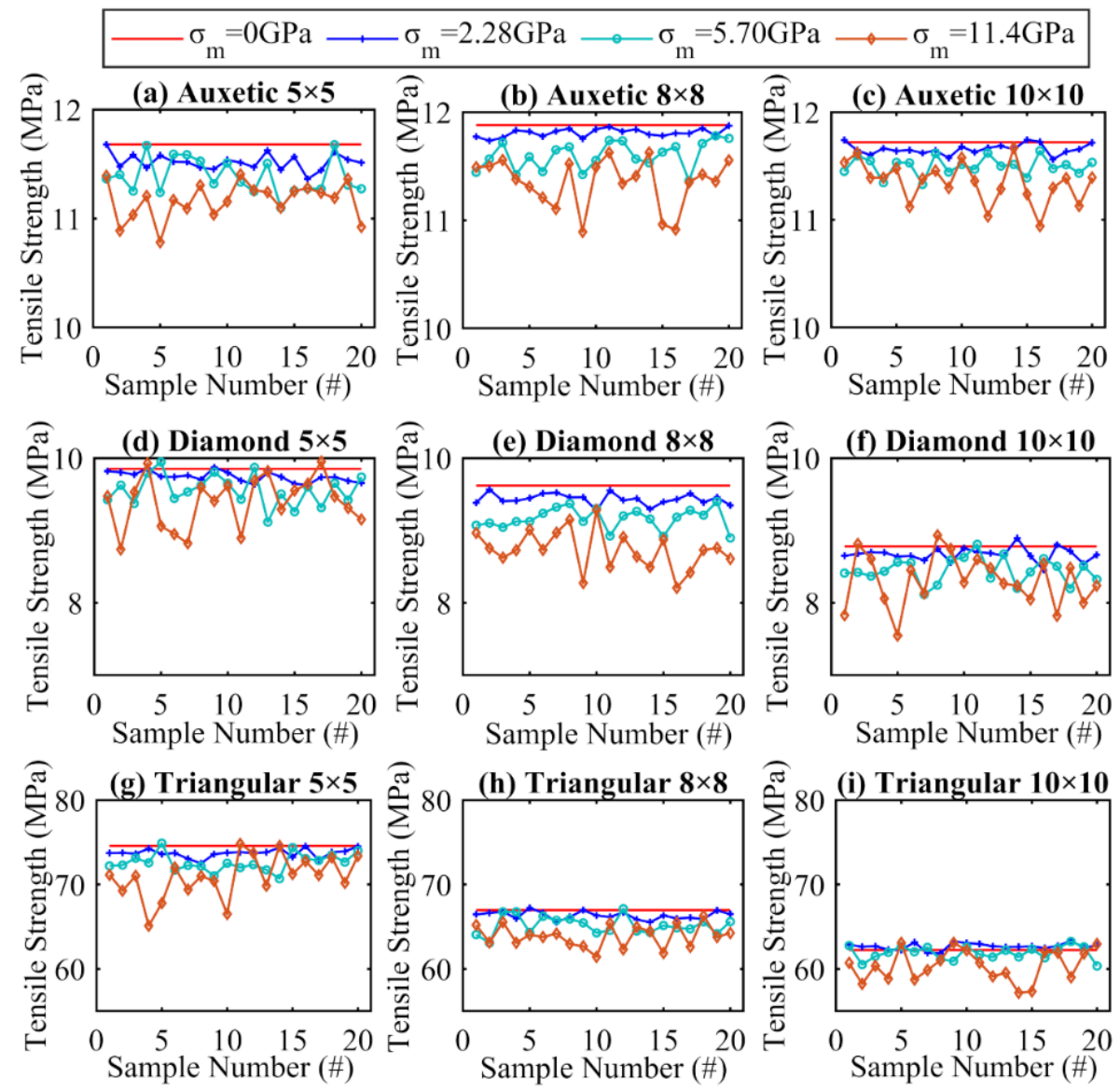

Fig. 5. 15 Tensile strength calculation results for different designs with material modulus variability

In comparison, the results for the structural elastic modulus (Fig. 5.16) suggest that the structural elastic modulus exhibit relatively small decrease with the increase of the 
material elastic modulus variabilities. The different sensitivity to local material property variability could be conceptually explained that while elastic modulus describes the overall "averaged" response of the structures to stress within the elastic regions, tensile strength reflects the "worst case" stress status within the structures.

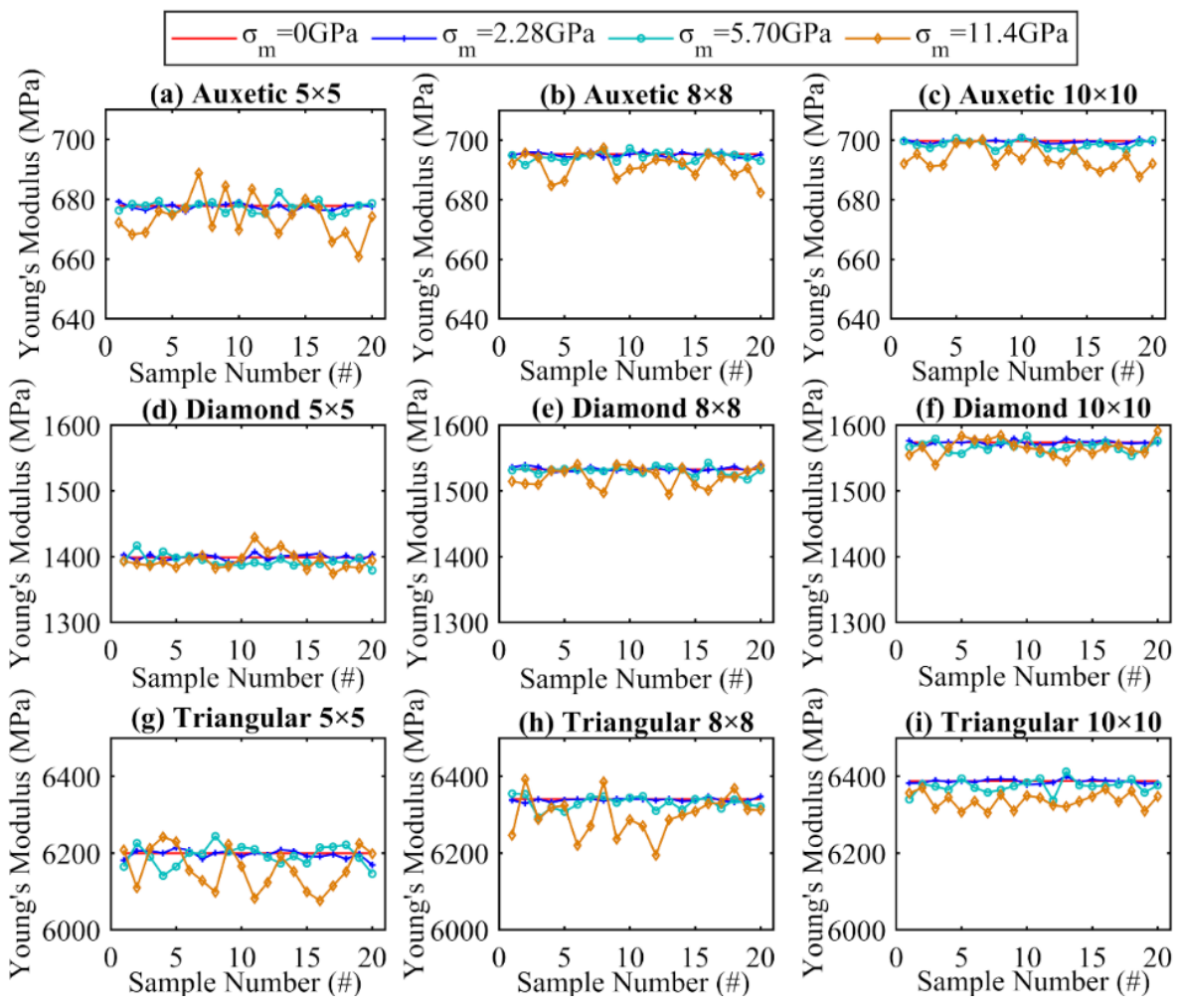

Fig. 5. 16 Elastic modulus calculation results for different designs with material modulus variability

Fig. 5.17 and Fig. 5.18 further illustrate the average and standard deviation values of the tensile strength and elastic modulus of each design, respectively. From Fig. 5.17(ac), the average tensile strength of all three types of cellular designs are influenced by both the material modulus variability and the pattern sizes, although the overall magnitude of effect for material modulus variability is small, particularly for the auxetic structure. In addition, the auxetic structure again exhibits smallest pattern size effects. The effects of 
material modulus variability on the tensile strength of cellular structures appear to be linear and independent to the structure pattern size, which can be expressed as:

$$
\sigma_{\text {tensile }}=\sigma_{\text {Baseline }}-C_{2} * \sigma_{m}
$$

where $\sigma_{\text {tensile }}$ is the average tensile strength of the structure, and $\sigma_{\text {Baseline }}$ is the baseline tensile strength of the structure with perfect material. From linear regression fitting analysis, the values of $C_{2}$ were obtained as $0.033,0.040$ and 0.1750 , with the correlations $\left(R^{2}\right)$ of $86.19 \%, 82.18 \%$ and $84.59 \%$ for auxetic, diamond and triangular structures, respectively. Comparing the values of $C_{2}$ from Equation (5.2) and the values of $C_{1}$ from Equation (5.1), the effect of material elastic modulus on the structural strength is mostly negligible compared to the effect of material strength, but should not be entirely overlooked in certain design cases. For example, the tensile strengths of the cellular structures exhibit a decrease of $4.5 \%, 4.7 \%$ and $4.9 \%$ for the auxetic, diamond and triangular designs, respectively, at the material modulus variability level of $\sigma_{m}=11.4 \mathrm{GPa}(10 \%)$. In addition, the triangular structure also exhibits the highest structural strength sensitivity to material elastic modulus. This might be resulted from the fact that for the stretching-dominated structures the local fluctuation of the elastic modulus could potentially result in occurrence of significant bending deformations, which leads to more significant stress concentration effects. 


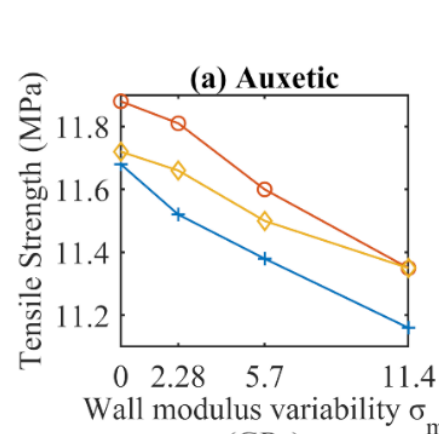

(GPa)
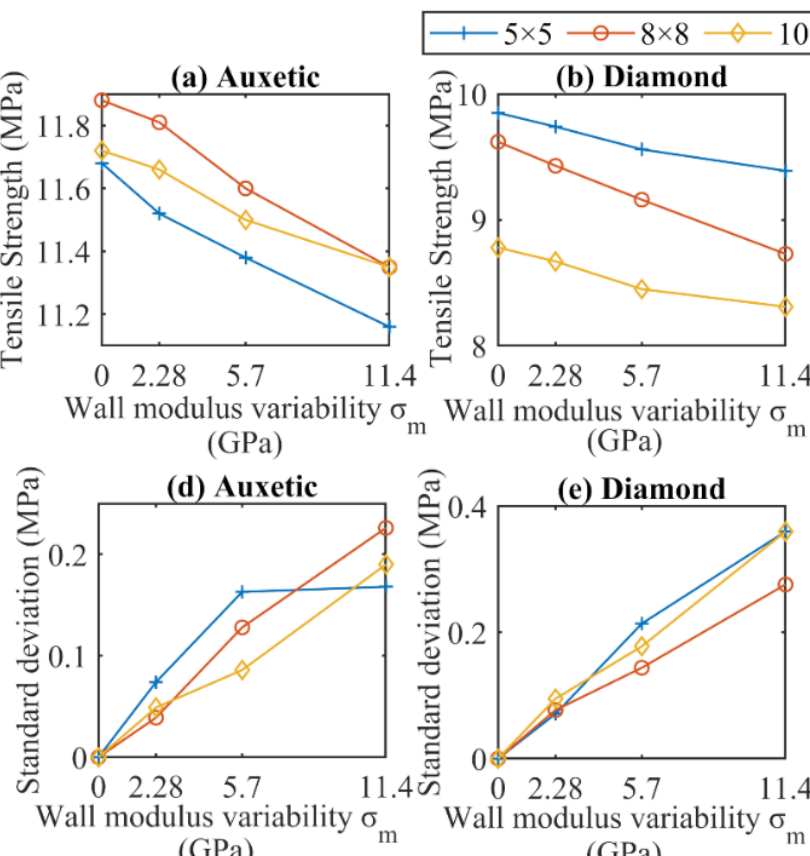

(GPa)

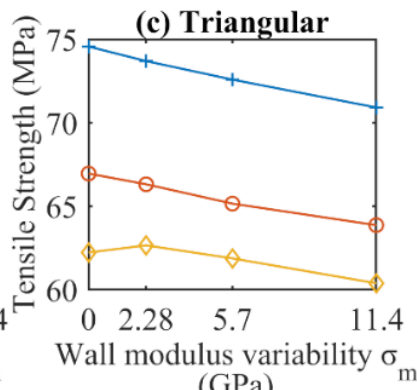

(GPa)

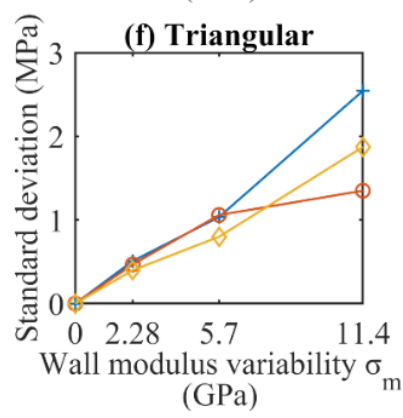

Fig. 5. 17 The relationships between the material modulus variability and mean (a, b, c) / standard deviation $(\mathrm{d}, \mathrm{e}, \mathrm{f}))$ of the structural tensile strengths for different designs

From Fig. 5.18, the average elastic modulus of the cellular structures exhibits relatively low sensitivity to the material elastic modulus variability. The average elastic modulus of the auxetic, diamond and triangular structures all exhibit decrease of $<1 \%$ when the cell wall elastic modulus variability is at $11.4 \mathrm{GPa}$ level (10\%). This observation is again conceptually intuitive, as the elastic modulus of the cellular structures can be considered as an evaluation of its elastic characteristics "averaged" throughout all the individual components. On the other hand, the effect of pattern size is more significant, which was previously discussed in Section 3.2 and therefore will not be further elaborated here.

Furthermore, for the standard deviation analysis, results from Fig. 5.17(d-f) and Fig. 5.18(d-f) both suggest that the structural elastic modulus exhibit lower scattering than the imperfection at the material level, although there clearly exists a near-linear trend between the variabilities at the two scales. The effect of pattern size, on the other hand, appears 
insignificant, which is consistent to the observations with the structural strength variability (Fig. 5.6(d-f)). More quantitative analysis might be needed to understand the mechanism of pattern size inhibition with the structural-level property variabilities, which is almost entirely absent from the existing knowledge base.
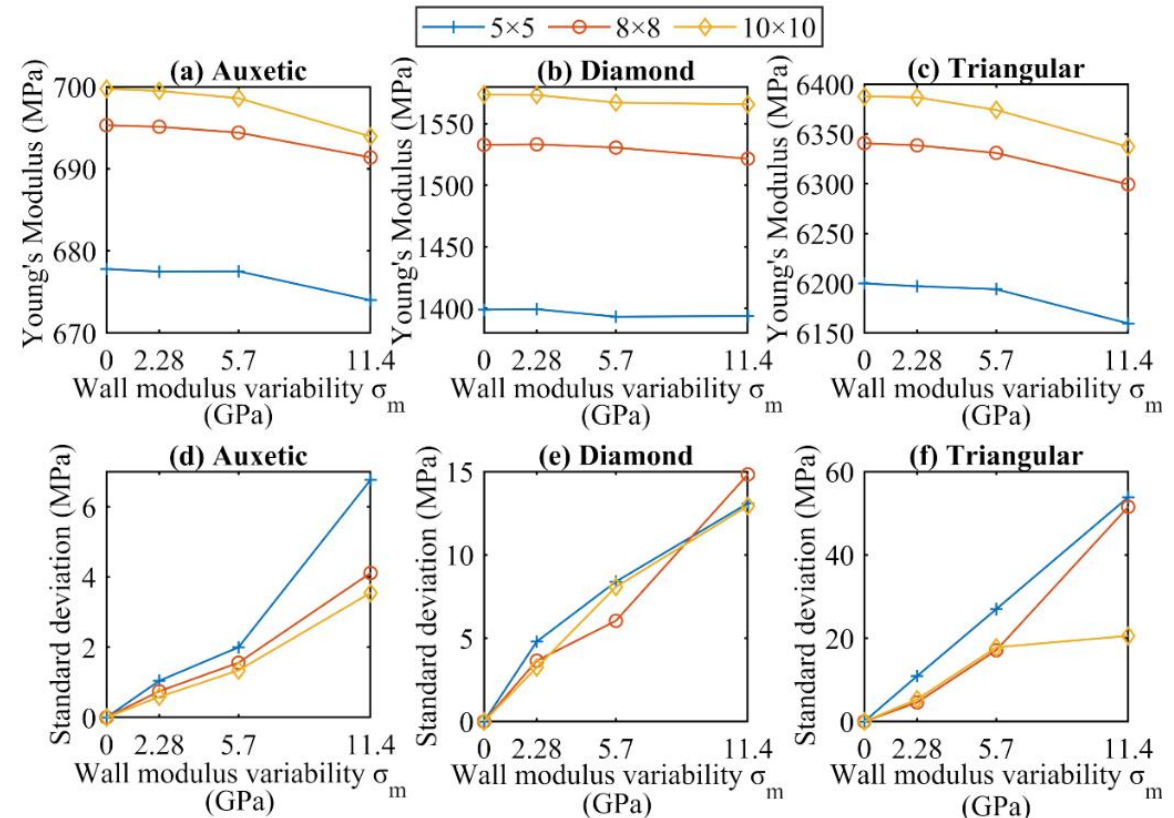

Fig. 5. 18 The relationships between the material elastic modulus variability and mean (a, $\mathrm{b}, \mathrm{c})$ / standard deviation (d, e, f)) of the elastic modulus for different designs

The multi-step failure characteristics of three structures can also be obtained through the strain-stress curves, which is similar to that in Section 5.3.2. Table 5.4 shows the stepwise failure strength and elastic modulus of the auxetic structures under different levels of wall modulus variabilities. From the results, the wall modulus variability has relatively insignificant effect on the stepwise properties compared to the wall strength variability. Both tensile strength and Young's modulus of the structures are relatively constant with varying wall modulus variabilities. 
Table 5. 4 Average tensile strength and Young's modulus of auxetic structures with different unit cell numbers and wall modulus variabilities at each failure step

\begin{tabular}{|c|c|c|c|c|}
\hline & \multirow[b]{2}{*}{$\sigma_{s}$} & \multirow[b]{2}{*}{$\sigma_{m}$} & \multicolumn{2}{|c|}{ Strength (MPa) } \\
\hline & & & Step 1 & Step 2 \\
\hline & $\mathbf{0}$ & $\mathbf{0}$ & 11.68 & 0.99 \\
\hline Auxetic & 0 & 2.28 & $11.51 \pm 0.07$ & $0.99 \pm 0.01$ \\
\hline \multirow[t]{3}{*}{$5 \times 5$} & 0 & 5.70 & $11.38 \pm 0.16$ & $1.27 \pm 0.44$ \\
\hline & 0 & 11.4 & $11.16 \pm 0.17$ & $1.17 \pm 0.37$ \\
\hline & $\mathbf{0}$ & $\mathbf{0}$ & 11.88 & 0.69 \\
\hline Auxetic & 0 & 2.28 & $11.81 \pm 0.04$ & $0.63 \pm 0.21$ \\
\hline \multirow[t]{3}{*}{$8 \times 8$} & 0 & 5.70 & $11.6 \pm 0.13$ & $0.57 \pm 0.32$ \\
\hline & 0 & 11.4 & $11.35 \pm 0.23$ & $0.57 \pm 0.45$ \\
\hline & $\mathbf{0}$ & $\mathbf{0}$ & 11.72 & --- \\
\hline Auxetic & 0 & 2.28 & $11.66 \pm 0.05$ & --- \\
\hline \multirow[t]{5}{*}{$10 \times 10$} & 0 & 5.70 & $11.5 \pm 0.09$ & --- \\
\hline & 0 & 11.4 & $11.35 \pm 0.19$ & --- \\
\hline & & & \multicolumn{2}{|c|}{ Modulus (MPa) } \\
\hline & $\sigma_{s}$ & $\sigma_{m}$ & Step 1 & Step 2 \\
\hline & $\mathbf{0}$ & $\mathbf{0}$ & 677.8 & 41.2 \\
\hline Auxetic & 0 & 2.28 & $677.47 \pm 1.04$ & $41.16 \pm 0.31$ \\
\hline \multirow[t]{3}{*}{$5 \times 5$} & 0 & 5.70 & $677.51 \pm 2$ & $56.97 \pm 24.6$ \\
\hline & 0 & 11.4 & $673.99 \pm 6.76$ & $51.23 \pm 19.86$ \\
\hline & $\mathbf{0}$ & $\mathbf{0}$ & 695.3 & 36.5 \\
\hline Auxetic & 0 & 2.28 & $695.12 \pm 0.75$ & $32.88 \pm 11.25$ \\
\hline \multirow[t]{3}{*}{$8 \times 8$} & 0 & 5.70 & $694.41 \pm 1.56$ & $30.65 \pm 17.57$ \\
\hline & 0 & 11.4 & $691.39 \pm 4.11$ & $32.32 \pm 26.21$ \\
\hline & $\mathbf{0}$ & $\mathbf{0}$ & 699.8 & $-\cdots$ \\
\hline Auxetic & 0 & 2.28 & $699.5 \pm 0.59$ & --- \\
\hline \multirow[t]{2}{*}{$10 \times 10$} & 0 & 5.70 & $698.6 \pm 1.34$ & --- \\
\hline & 0 & 11.4 & $693.94 \pm 3.55$ & --- \\
\hline
\end{tabular}


Table 5.5 shows the stepwise failure strength and elastic modulus of the diamond structures under different levels of wall modulus variabilities. Similar to the results of the auxetic structure, the stepwise properties of the diamond structures do not exhibit significant dependency on the wall modulus variabilities during the first 3 steps. The results for both the auxetic and the diamond structures might be associated with the non-rigid structural architecture of the bending-dominated designs, whose deformation mode is relatively less impacted by local damages.

Table 5.5 Average tensile strength and Young's modulus of diamond structures with different unit cell numbers and wall strength variabilities at each failure step

\begin{tabular}{|c|c|c|c|c|c|c|c|}
\hline & \multirow[b]{2}{*}{$\sigma_{s}$} & \multirow[b]{2}{*}{$\sigma_{m}$} & \multicolumn{5}{|c|}{ Strength $(\mathrm{MPa})$} \\
\hline & & & Step 1 & Step 2 & Step 3 & Step 4 & Step 5 \\
\hline \multirow{4}{*}{ Diamond $5 \times 5$} & $\mathbf{0}$ & $\mathbf{0}$ & 9.85 & 7.39 & --- & -- & --- \\
\hline & 0 & 2.28 & $9.74 \pm 0.07$ & $7.33 \pm 0.05$ & --- & --- & --- \\
\hline & 0 & 5.70 & $9.56 \pm 0.21$ & $7.71 \pm 0.52$ & $6.03 \pm 2.17$ & $3.72 \pm 2.5$ & $1.77 \pm 2.06$ \\
\hline & 0 & 11.4 & $9.39 \pm 0.36$ & $7.92 \pm 0.72$ & $6.20 \pm 1.59$ & $3.57 \pm 2.9$ & $1.44 \pm 1.85$ \\
\hline \multirow{4}{*}{ Diamond $8 \times 8$} & $\mathbf{0}$ & $\mathbf{0}$ & 9.62 & --- & --- & --- & -- \\
\hline & 0 & 2.28 & $9.43 \pm 0.08$ & --- & --- & --- & --- \\
\hline & 0 & 5.70 & $9.16 \pm 0.14$ & $5.58 \pm 4.30$ & --- & --- & --- \\
\hline & 0 & 11.4 & $8.73 \pm 0.28$ & $7.23 \pm 2.84$ & $4.86 \pm 3.68$ & $3.33 \pm 3.46$ & --- \\
\hline \multirow{6}{*}{ Diamond $10 \times 10$} & $\mathbf{0}$ & $\mathbf{0}$ & 8.78 & 8.48 & 8.47 & & \\
\hline & 0 & 2.28 & $8.67 \pm 0.09$ & $8.58 \pm 0.07$ & $8.48 \pm 0.07$ & $8.4 \pm 0.08$ & $7.98 \pm 1.88$ \\
\hline & 0 & 5.70 & $8.45 \pm 0.18$ & $8.5 \pm 0.22$ & $7.41 \pm 2.82$ & $4.77 \pm 4.2$ & --- \\
\hline & 0 & 11.4 & $8.31 \pm 0.36$ & $8.39 \pm 0.36$ & $7.73 \pm 1.99$ & $6.21 \pm 3.15$ & $3.18 \pm 3.45$ \\
\hline & & & \multicolumn{5}{|c|}{ Modulus (MPa) } \\
\hline & $\sigma_{s}$ & $\sigma_{m}$ & Step 1 & Step 2 & Step 3 & Step 4 & Step 5 \\
\hline \multirow{4}{*}{ Diamond $5 \times 5$} & $\mathbf{0}$ & $\mathbf{0}$ & 1398.9 & 992.6 & --- & -- & -- \\
\hline & 0 & 2.28 & $1399.1 \pm 4.8$ & $992.6 \pm 3.2$ & --- & --- & --- \\
\hline & 0 & 5.70 & $1393.1 \pm 8.4$ & $1088 \pm 109.3$ & $824.7 \pm 297.6$ & $501 \pm 336.3$ & $236.2 \pm 276.1$ \\
\hline & 0 & 11.4 & $1393 \pm 13.1$ & $1128 \pm 111.5$ & $847.1 \pm 226.7$ & $475 \pm 382.5$ & $188.7 \pm 243.5$ \\
\hline
\end{tabular}




\begin{tabular}{cccccccc}
\hline & $\mathbf{0}$ & $\mathbf{0}$ & $\mathbf{1 5 3 2 . 8}$ & --- & --- & --- & --- \\
Diamond 8×8 & 0 & 2.28 & $1533.2 \pm 3.6$ & --- & --- & --- & --- \\
& 0 & 5.70 & $1530.5 \pm 6.0$ & $916.5 \pm 706.4$ & --- & --- & --- \\
& 0 & 11.4 & $1521 \pm 14.8$ & $1220.8 \pm 478$ & $807.2 \pm 615.8$ & $542.6 \pm 570$ & \\
\hline \multirow{2}{*}{ Diamond 10×10 } & $\mathbf{0}$ & $\mathbf{0}$ & $\mathbf{1 5 7 3 . 7}$ & $\mathbf{1 5 0 3 . 6}$ & $\mathbf{1 4 3 4 . 8}$ & --- & --- \\
& 0 & 2.28 & $1573.2 \pm 3.2$ & $1537.8 \pm 3.1$ & $1502.8 \pm 3.0$ & $1468.4 \pm 2.9$ & $1362.6 \pm 320$ \\
& 0 & 5.70 & $1567.1 \pm 8.1$ & $1531.1 \pm 7.92$ & $129 \pm 493.5$ & $826 \pm 727.7$ & --- \\
& 0 & 11.4 & $1565.7 \pm 13$ & $1529.4 \pm 12.7$ & $1370.8 \pm 359$ & $1072 \pm 553.3$ & $539.6 \pm 539$ \\
\hline
\end{tabular}

5.3.2.2 Effect of cell wall modulus variability on failure response

The effect of the unit cell numbers and cell wall modulus variability on energy absorption of the structures was also studied. The energy absorption of individual runs for each types of structures are shown in Fig. 5.19. For the auxetic and triangular structures, the energy absorption generally decreases as the wall modulus variability increases. On the other hand, the diamond structures appear to not exhibit obvious trend as the wall modulus variability varies. 


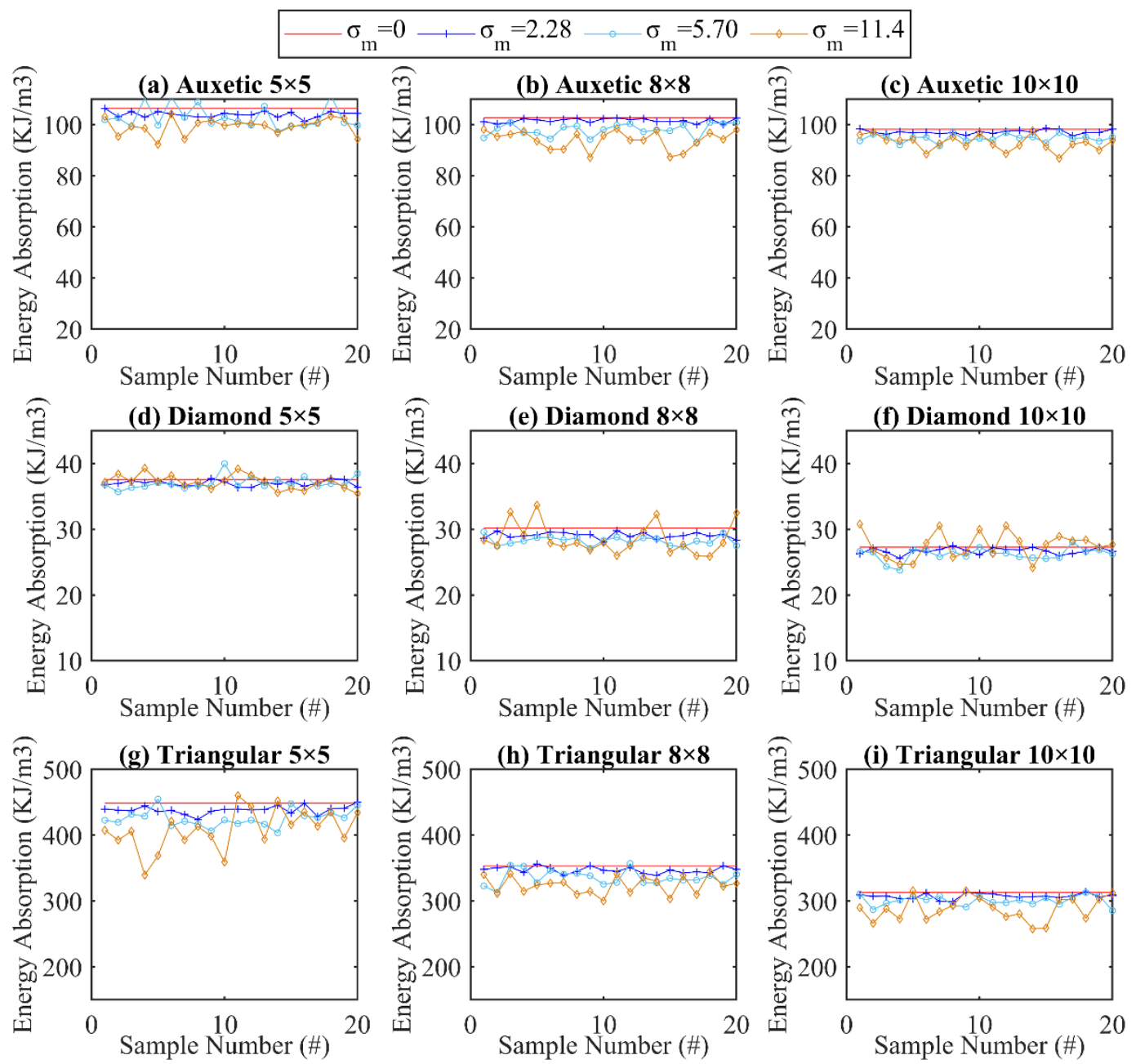

Fig. 5. 19 Energy absorption distribution of different designs

Fig. 5.20 further illustrate these observations, which could be explained from the results with both the initial failure strength (Fig.5.17) and elastic modulus (Fig. 5.18) of the different structures. From Fig. 5.20, it can be seen that the energy absorption of each types of unit cell design could be expressed as a linear function of the wall elastic modulus variability. Among three designs, the material modulus variability rarely affects the diamond structure. The triangular structure is the most sensitive structure to the material modulus variability. 

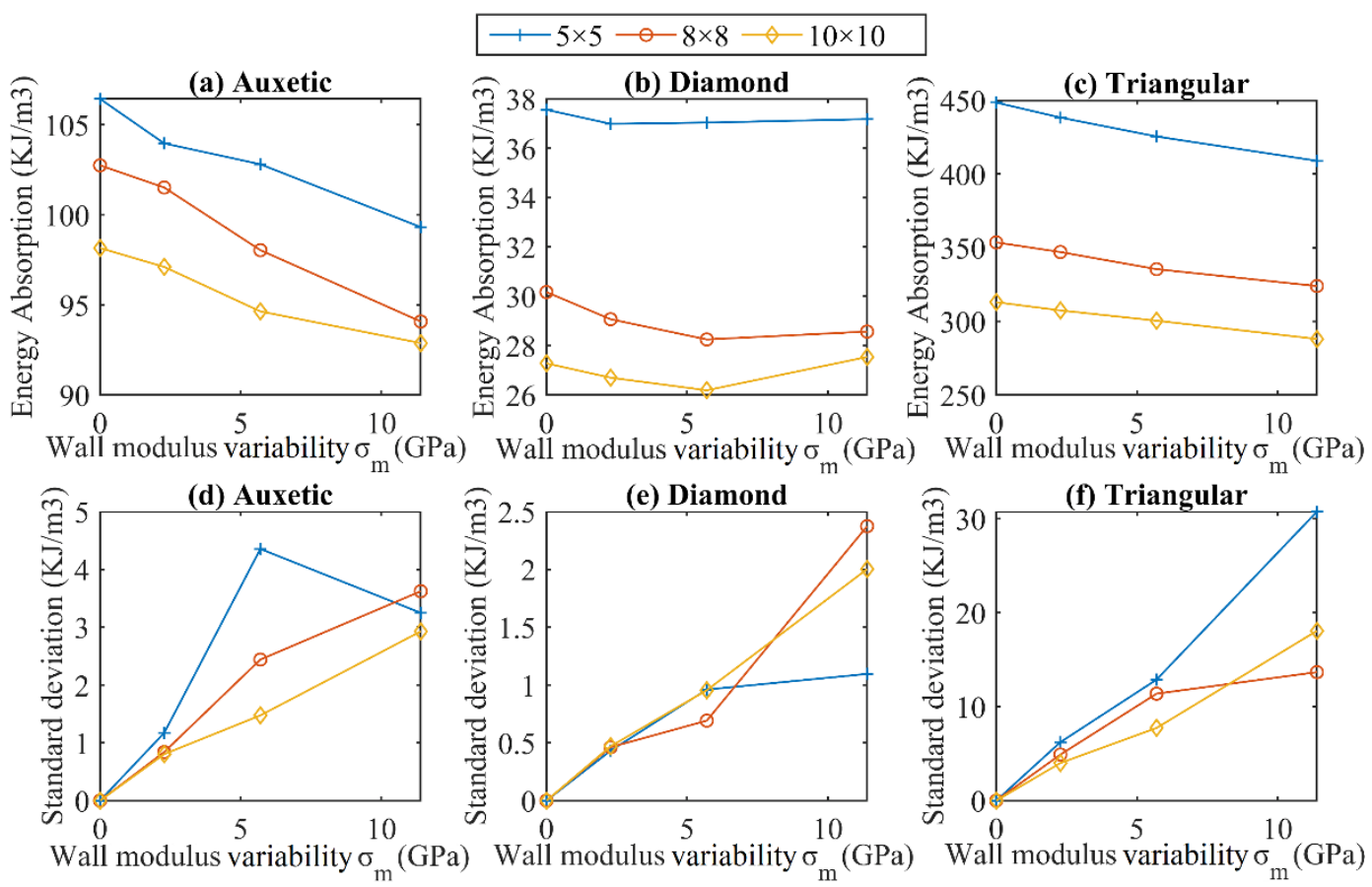

Fig. 5. 20 The relationships between the wall strength variability levels and mean (a, b c) / standard deviation $(\mathrm{d}, \mathrm{e}, \mathrm{f})$ of cellular energy absorption of different designs

Fig. 5.21-23 show the failure patterns of the three types of cellular designs with different unit cell numbers and cell wall modulus variabilities. Overall, the cell wall modulus variability influences the failure patterns of the cellular structures in similar ways as the cell wall strength variability but at less significant levels. For example, for the auxetic structures, at 10\% wall modulus variability the structures exhibit less frequent side-to-side failure patterns compared to the structures with $10 \%$ wall strength variability. 
(a) $\sigma_{\mathrm{s}}=0, \quad \sigma_{\mathrm{m}}=2.28$

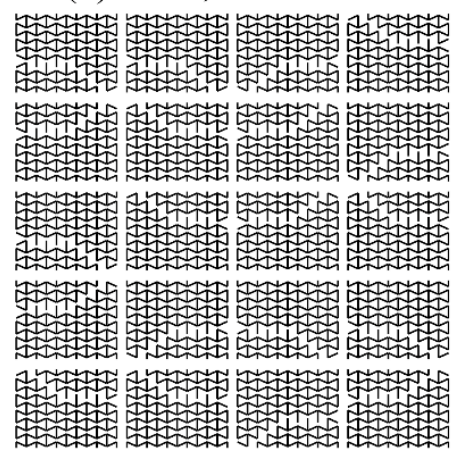

(d) $\sigma_{\mathrm{s}}=0, \sigma_{\mathrm{m}}=2.28$

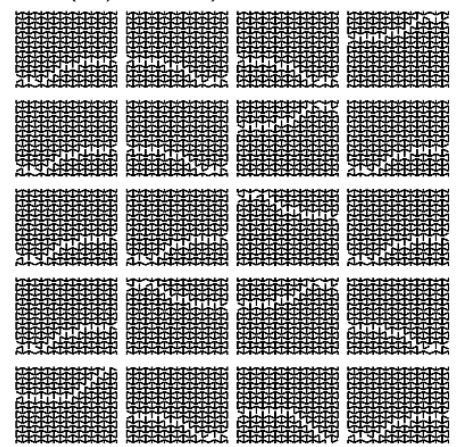

(g) $\sigma_{\mathrm{s}}=0.02, \sigma_{\mathrm{m}}=0$

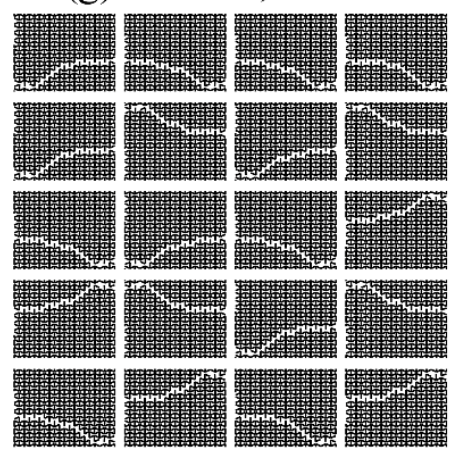

(b) $\sigma_{s}=0, \sigma m=5.70$

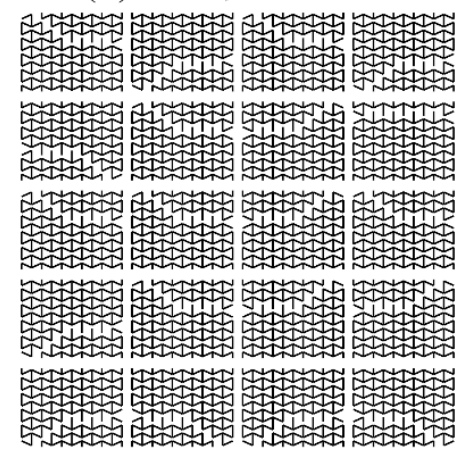

(e) $\sigma_{\mathrm{s}}=0, \sigma_{\mathrm{m}}=5.70$

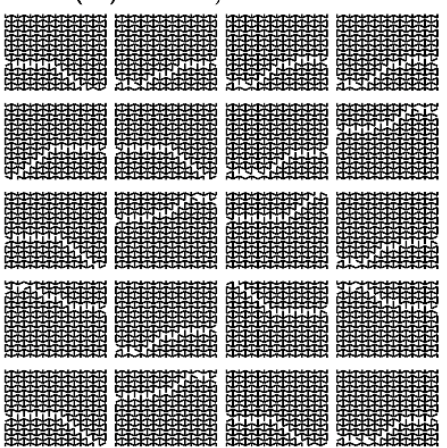

(h) $\sigma_{\mathrm{s}}=0.05, \sigma_{\mathrm{m}}=0$

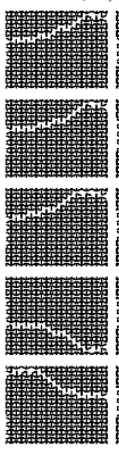

(c) $\sigma_{\mathrm{s}}=0, \sigma_{\mathrm{m}}=11.4$

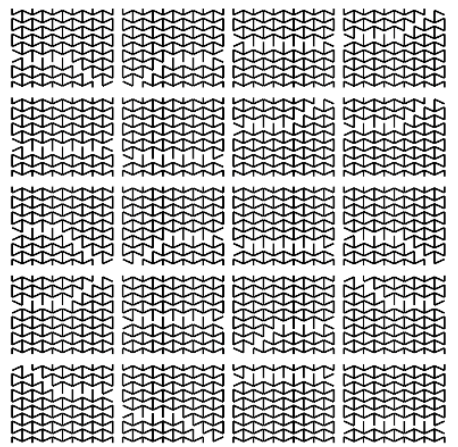

(f) $\sigma_{\mathrm{s}}=0, \sigma_{\mathrm{m}}=11.4$

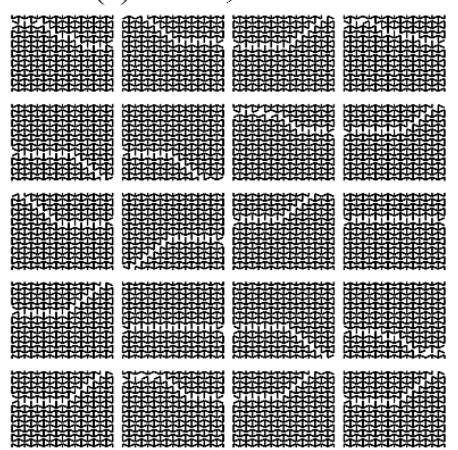

(i) $\sigma_{\mathrm{s}}=0.10, \sigma_{\mathrm{m}}=0$
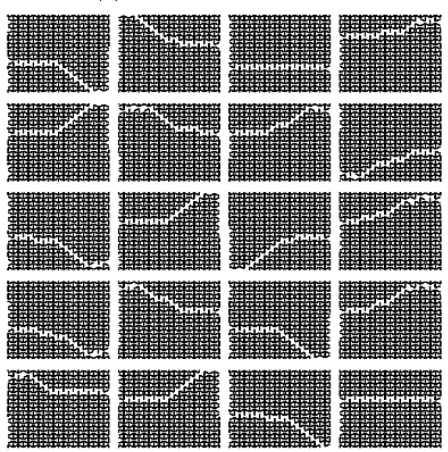

Fig. 5.21 The failure patterns of the auxetic structures with different unit cell numbers and wall modulus variability 
(a) $\sigma_{\mathrm{s}}=0, \sigma_{\mathrm{m}}=2.28$

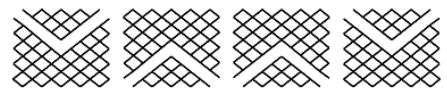

m $m$ m
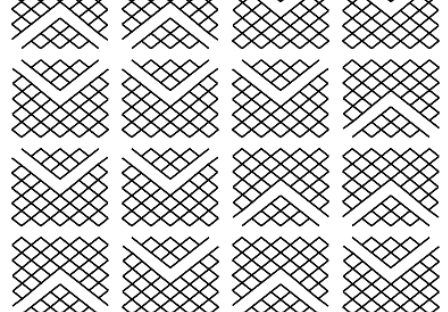

$$
\text { (d) } \sigma_{\mathrm{s}}=0, \sigma \mathrm{m}=2.28
$$

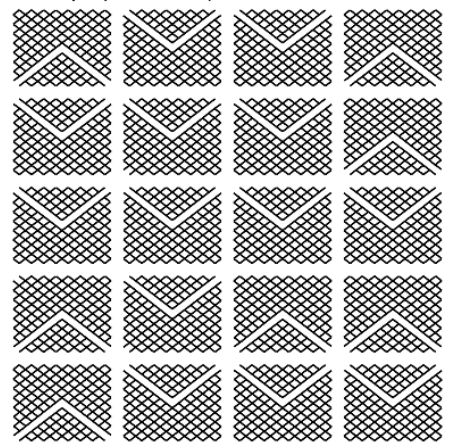

$$
\text { (g) } \sigma \mathrm{s}=0.02, \sigma \mathrm{m}=0
$$

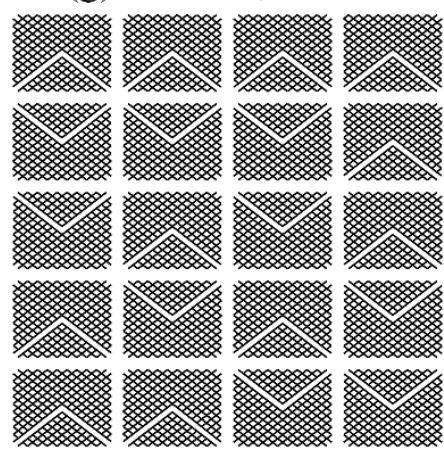

(b) $\sigma_{\mathrm{s}}=0, \sigma \mathrm{m}=5.70$

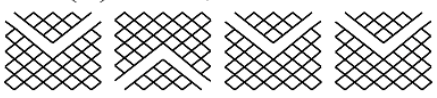

XX1

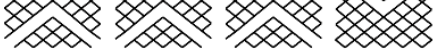

1)
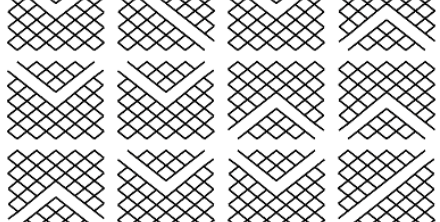

(e) $\sigma_{\mathrm{s}}=0, \sigma_{\mathrm{m}}=5.70$

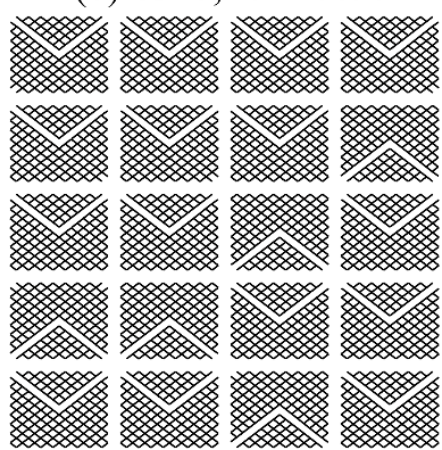

(h) $\sigma_{\mathrm{s}}=0.05, \sigma_{\mathrm{m}}=0$
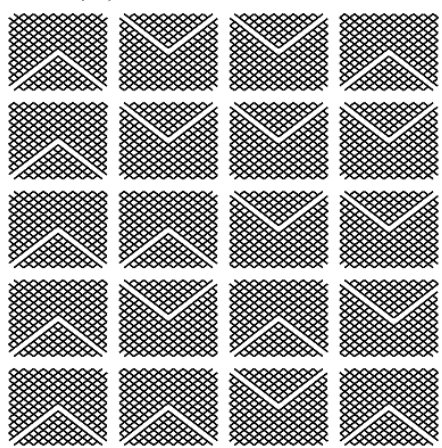

(c) $\sigma_{\mathrm{s}}=0, \sigma_{\mathrm{m}}=11.4$

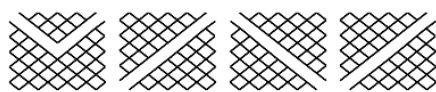

1) $12 \times 8$, W 3 m m y y s sys \%
(f) $\sigma \mathrm{s}=0, \sigma \mathrm{m}=11.4$

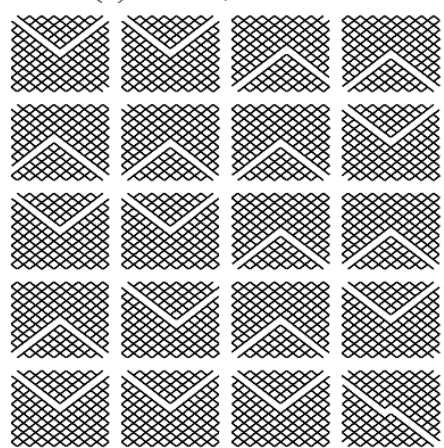

(i) $\sigma_{\mathrm{s}}=0.10, \sigma_{\mathrm{m}}=0$

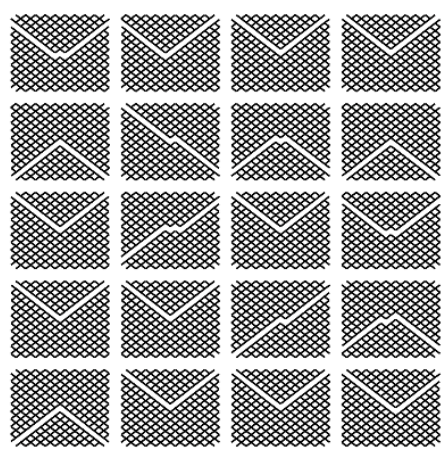

Fig. 5. 22 The failure patterns of the diamond structures with different unit cell numbers and wall modulus variability 
(a) $\sigma_{\mathrm{s}}=0, \sigma \mathrm{m}=2.28$

$\quad$ \% \% \%

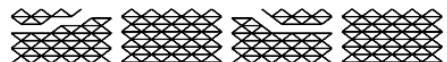

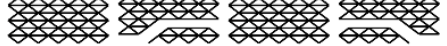

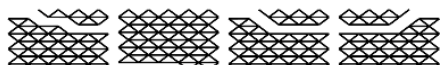

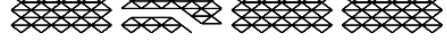
28 $20 \%$ \& $\quad \%$ W $\quad \square \quad \square$
(d) $\sigma_{\mathrm{s}}=0, \quad \sigma_{\mathrm{m}}=2.28$

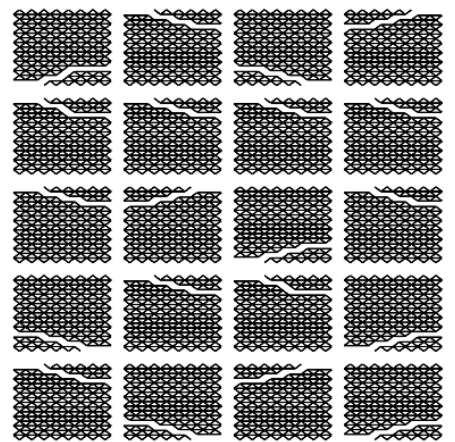

$(\mathrm{g}) \sigma_{\mathrm{s}}=0.02, \sigma_{\mathrm{m}}=0$

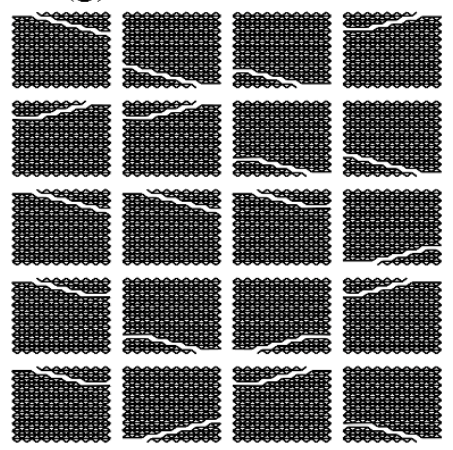

(b) $\sigma_{\mathrm{s}}=0, \quad \sigma \mathrm{m}=5.70$
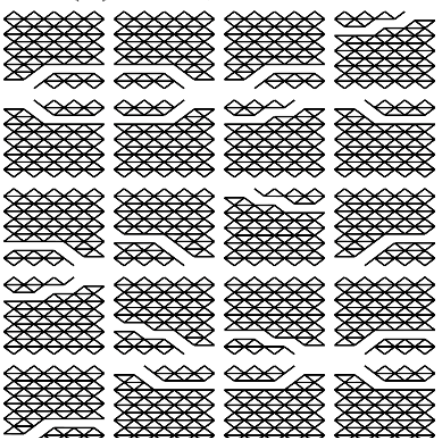

(e) $\sigma_{\mathrm{s}}=0, \sigma_{\mathrm{m}}=5.70$

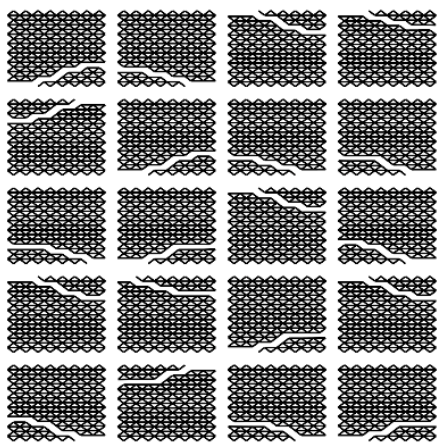

(h) $\sigma_{\mathrm{s}}=0.05, \quad \sigma_{\mathrm{m}}=0$

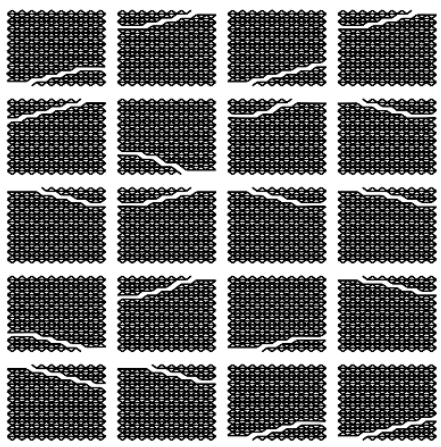

(c) $\sigma_{\mathrm{s}}=0, \sigma_{\mathrm{m}}=11.4$

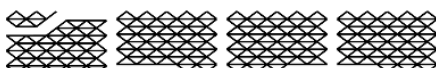

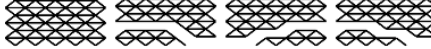

$\$ \forall$ W

$\square \quad \square$
$\quad \square \quad$

W \& \&

$\begin{array}{lll}\quad & \quad\end{array}$

\& $\quad$ \& W

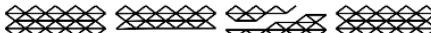
W

(f) $\sigma_{\mathrm{s}}=0, \sigma_{\mathrm{m}}=11.4$

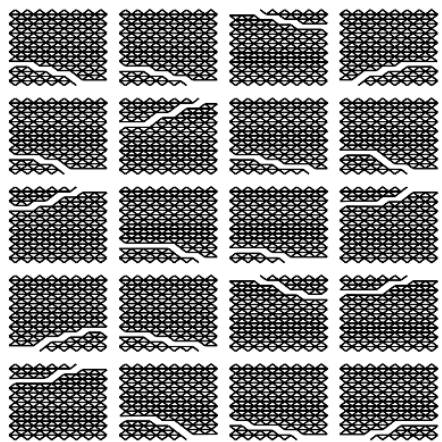

(i) $\sigma_{\mathrm{s}}=0.10, \sigma \mathrm{m}=0$

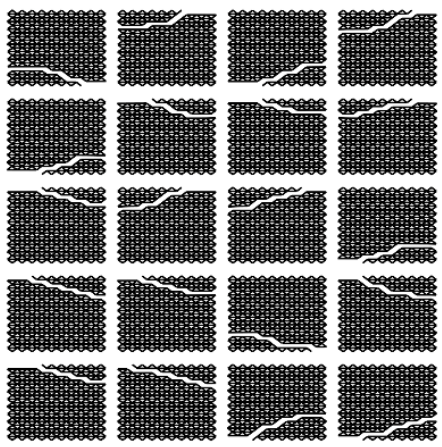

Fig. 5. 23 The failure patterns of the triangular structures with different unit cell numbers and wall modulus variability

\subsubsection{Combine effect}

Lastly, the combined effects of wall strength and wall modulus variabilities was studied. This was considered to be more representative to real-world situations, where the materials are likely to exhibit property variabilities with both the tensile strength and elastic modulus. For this study, the same three levels of variabilities $(2 \%, 5 \%, 10 \%)$ for wall 
strength and wall modulus were investigated, which resulted in 9 different combinations. The study was carried out in the same manner with 20 calculation runs for each types of sample designs. The pattern size for the cellular structures was kept constant at $8 \times 8$, since from the previous studies it has been shown that the effect of pattern size is independent and thus decouple-able.

(a) Auxetic

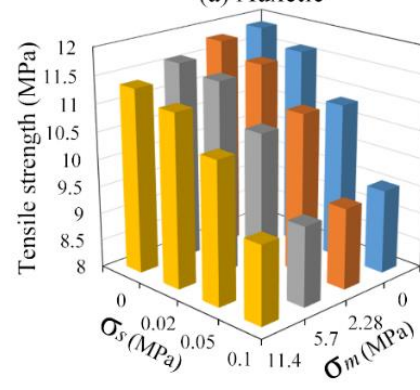

(d) Auxetic

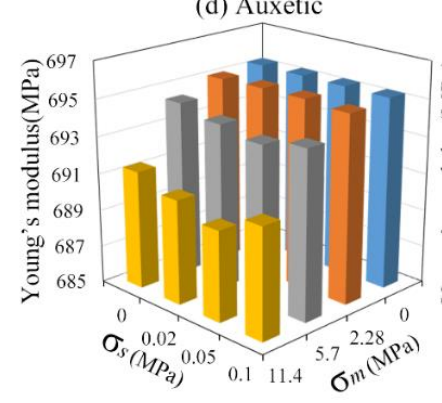

(b) Diamond

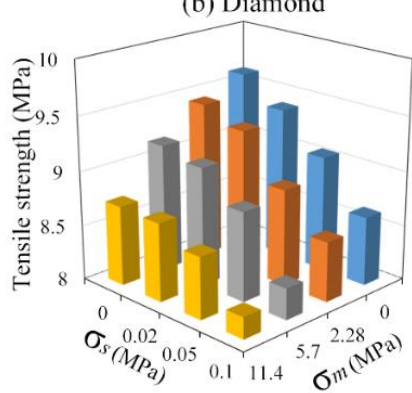

(e) Diamond

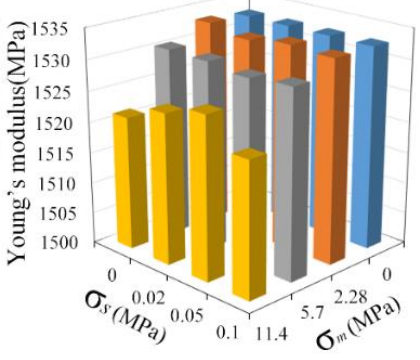

(c) Triangular

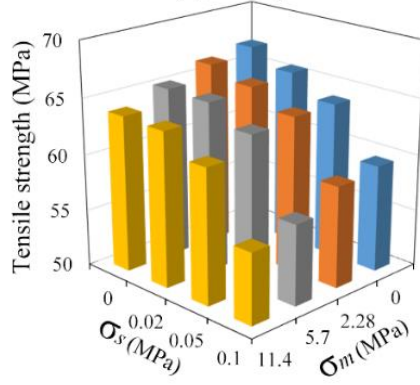

(f) Triangular

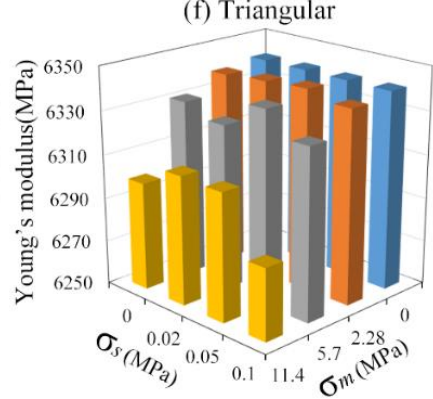

Fig. 5.24 The averaged mechanical properties of different cellular structures with wall strength and elastic modulus variabilities

Fig.5.24 shows the averaged results for both the tensile strength and Young's modulus of different structures. It is readily seen that both tensile strength and Young's modulus increase as the wall strength variability and wall modulus variability increase. The structural tensile strength follows a linear relationship with both the wall strength variability and the wall modulus variability, which is further illustrated in Fig. 5.25. Such relationship was found to be essentially a linear superposition of Equation (5.1) and Equation (5.2), which can be expressed as: 


$$
\sigma_{\text {tensile }}=\sigma_{\text {Baseline }}-C_{1} * \sigma_{s}-C_{2} * \sigma_{m}
$$

where $\sigma_{\text {tensile }}$ is the average value of the tensile strength of the structure, $\sigma_{\text {Baseline }}$ is the tensile strength of the structure with perfect material, and $C_{1}$ and $C_{2}$ take the same values as Equation (5.1) and Equation (5.2). Equation (5.3) was shown to achieve correlation levels $\left(R^{2}\right)$ of $99.28 \%, 92.88 \%$ and $95.70 \%$ for auxetic, diamond and triangular structures, respectively.
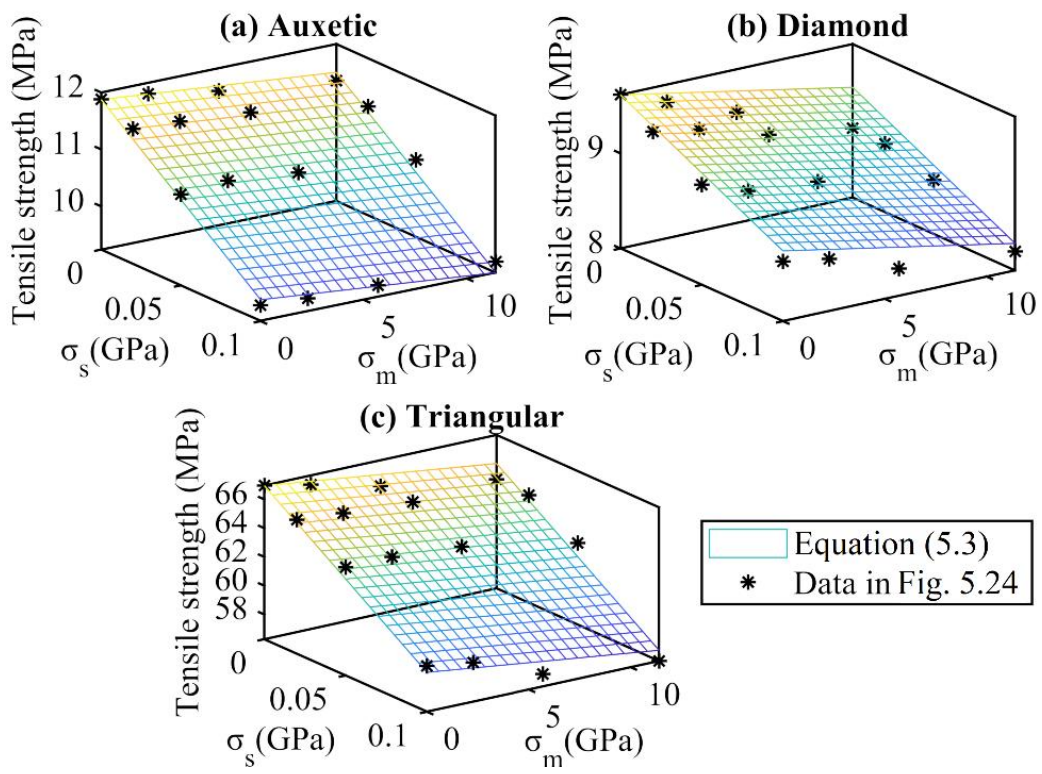

Fig. 5.25 Relationships between wall strength variability, wall modulus variability and tensile strength for different designs

\subsubsection{Discussion}

From the results discussed above, it can be concluded that the wall strength variability has more significant effect on the mechanical properties of the $2 \mathrm{D}$ cellular structures compared to the wall modulus variability. In this section, the analysis will be focused on the effect of the wall strength variability on the tensile strength of cellular structures (with a constant wall modulus variability of 0 ). Based on the calculation of our 
analytical model, the principle stress in each individual wall and the applied stress of the cellular structure are proportional to the applied strain and Young's modulus of the raw materials, shown in Equation (5.4) and Equation (5.5). In the equations, the $\sigma_{w a l l-i}$ is the principle stress level within walls $i$ and the $\sigma_{\text {lattice }}$ is the nominal stress applied to the cellular structures. $E$ is the Young's modulus of the raw materials and $\varepsilon$ is the applied displacement/strain loading to the cellular structure. $D$ and $D i$ are two coefficients at the structural and individual wall levels that are only dependent on the cellular geometry. It is noted that even within a cellular structure the values of $D i$ of individual walls could vary due to the inhomogeneity.

$$
\begin{aligned}
& \sigma_{\text {wall-i }}=D_{i} * E * \varepsilon \\
& \sigma_{\text {lattice }}=D * E * \varepsilon
\end{aligned}
$$

From Equation (5.4), when the principle stress $\sigma_{w a l l-i}$ of a critical wall (with corresponds coefficient $D_{\text {critical }}$ ) reaches the failure strength $\sigma_{\text {material }}$ of the solid materials, the cellular structure will fail under the brittle perfect-elastic material model. The corresponding $\sigma_{\text {lattice }}$ in Equation (5.5) will represent the tensile strength for the cellular structure, $\sigma_{\text {tensile }}$. Therefore, at this critical point, Equation (5.4) and (5.5) become

$$
\begin{aligned}
& \sigma_{\text {material }}=D_{\text {critical }} * E * \varepsilon_{\text {critical }}, \\
& \sigma_{\text {tensile }}=D * E * \varepsilon_{\text {critical }} .
\end{aligned}
$$

Rewrite Equation (5.6) and (5.7) into the relationship between the tensile strengths of the cellular structure $\sigma_{\text {tensile }}$ and the material $\sigma_{\text {material }}$ yields:

$$
\sigma_{\text {tensile }}=\frac{D}{D_{\text {critical }}} * \sigma_{\text {material }}
$$


It becomes clear that the strength of the cellular structures would exhibit the same type of variability distribution characteristics as the materials, with an additional scaling factor of $\frac{D}{D_{\text {critical }} \text {. }}$

In the case of 2D cellular structures, due to the symmetry of the geometries, there exist four identical walls with the same principal stress level when there are no material property variabilities. Therefore, when material property variability is considered, the determination of the critical wall becomes the problem of determining the minimum value of four independent variables with identical normal distribution functions, which is a classic problem from extreme value theory [143]. The numerical solution of such problem can be readily obtained, which is also a distribution function. Fig. 5.26 shows the calculated probability density curves of the resulting distributions for different structures with different levels of wall strength variability based on the $5 \times 5$ pattern size, accounting the four most critical walls identified from the analysis with perfect material properties. The probability density curves of wall strength variability for all three structures exhibit highly consistent patterns. The mean values of the tensile strength calculated based on these results are listed in Table 5.6.
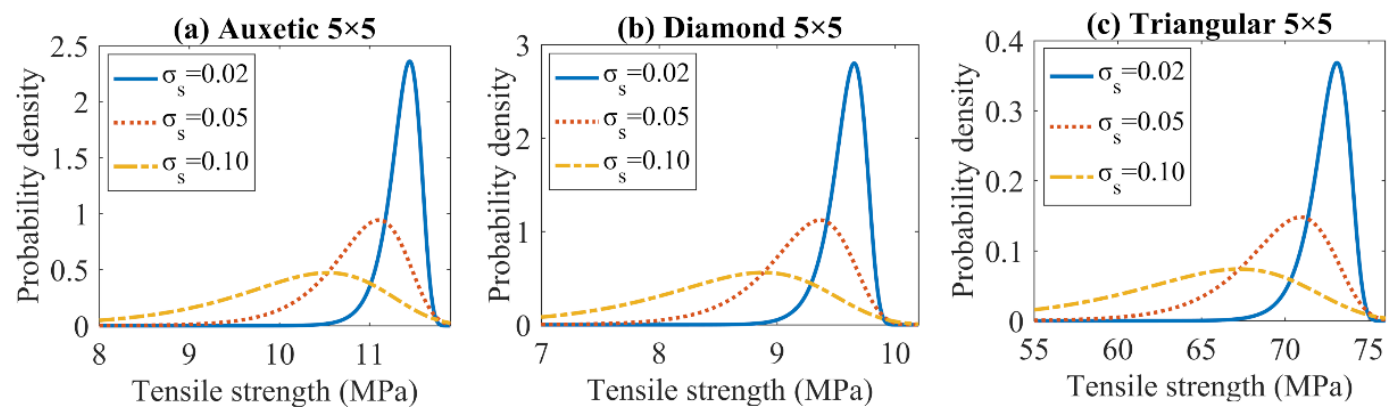

Fig. 5. 26 Probability density curves of tensile strength under different wall strength variability $\left(\sigma_{s}\right)$ for different structures considering the first four identical walls 
Table 5. 6 Tensile strength (MPa) for different structures under different wall strength variability $\left(\sigma_{s}\right)$ when considering the first four identical walls

\begin{tabular}{ccccc}
\hline Structure & \multicolumn{4}{c}{ Wall strength variability $\sigma_{s}(\mathrm{GPa})$} \\
\cline { 2 - 5 } & $\sigma_{s}=0$ & $\sigma_{s}=0.02$ & $\sigma_{s}=0.05$ & $\sigma_{s}=0.10$ \\
\hline Auxetic 5 $\times 5$ & 11.68 & 11.45 & 11.10 & 10.53 \\
Diamond 5 $\times 5$ & 9.85 & 9.65 & 9.36 & 8.88 \\
Triangular 5 $\times 5$ & 74.57 & 73.11 & 70.93 & 67.26 \\
\hline
\end{tabular}

From Table 5.6, the strength reduction ratios (tensile strength with wall variability divided by tensile strength without wall variability) for all three cellular structures exhibit the same magnitude as the wall strength variability levels $(2 \%, 5 \%$ and $10 \%$, or $\sigma_{s}=0.02 G P a, \sigma_{s}=0.05 G P a$ and $\left.\sigma_{s}=0.10 G P a\right)$. Compare the results from Table 5.6 with those from Fig. 5.6, the predictions from extreme value theory appear to agree well with the analytical modeling results for the diamond and triangular structures. On the other hand, there exist significant discrepancies for the auxetic structures. This was mostly attributed to the specific topological design of the auxetic structures, which, while exhibit minimal pattern size effects compared to the non-auxetic counterparts, also results in more homogeneous stress distributions and subsequently, more complex failure characteristics when there exist individual wall strength variabilities. To further understand this issue, Fig. 5.27 shows the most critical walls of each types of cellular topologies, indexed by their level of criticality under perfect material condition, i.e. in each topology, the most critical cell walls are labeled \#1, and the 7th critical cell walls are labeled \#7. Due to the symmetry of the structures, only a quadrant of each topologies is shown for clarity. In Fig. 5.27, the criticality level of the walls is expressed in the form of Equation (5.4), or as a function of the applied nominal displacement/strain. From Fig. 5.27, it becomes clear that for the 
auxetic structure, the stress levels of cell walls \#1 and \#2 differs by $\sim 1.8 \%$, whereas such difference is $\sim 14.09 \%$ and $\sim 7.0 \%$ for the diamond and triangular structures, respectively. In addition, for the auxetic structure, the differences of stress levels of the 7 most critical walls labeled in Fig. 5.27(b) are all smaller than 4.5\%. The direct implication of the small differences in criticality levels among these cell walls is that with even moderately small material strength variability they could all potentially become the most critical one.

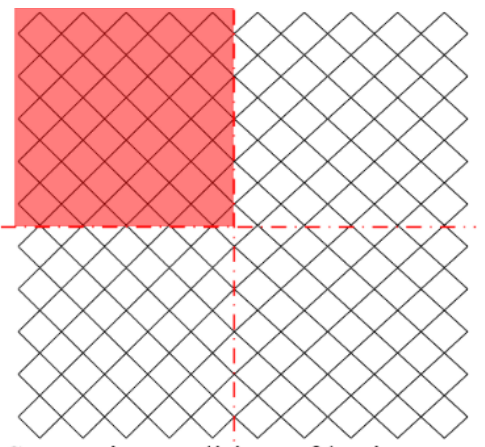

(a) Symetric condition of lattice structure

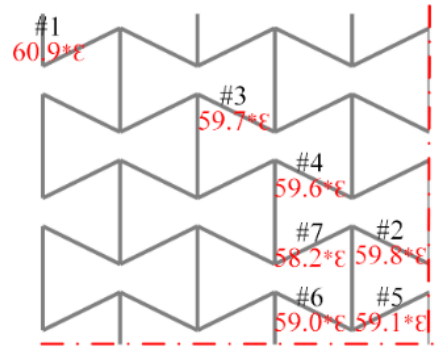

(b) Auxeic $5 \times 5$

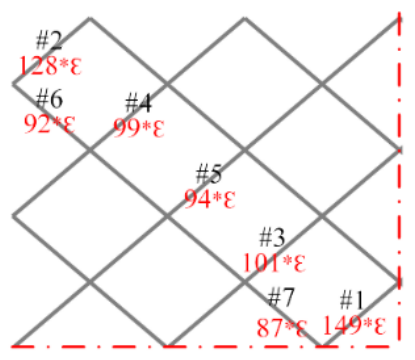

(c) Diamond $5 \times 5$

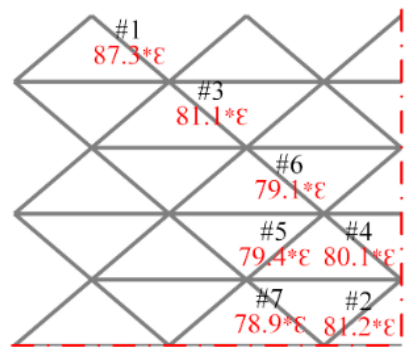

(d) Triangular $5 \times 5$

Fig. 5. 27 (a)The symetric conditon of cellular structure; (b,c,d) stress levels of top seven critial walls and the correponding locations of difference structures

Fig.5.28 illustrates the criticality probability densities of the \#1 and \#2 walls for the three types of cellular topologies, again based on $5 \times 5$ pattern size. For the auxetic structures, the probability densities of \#1 and \#2 walls exhibit significant overlap, which means that both sets of walls are subjected to similar levels of probabilities to become most critical. On the other hand, for the diamond structures, due to the large difference of the mean 
critical nominal stress levels between the distribution functions for \#1 and \#2 walls, even as material strength exhibits relatively large variability, the probability of \#1 walls to become more critical is still sufficiently high. The octahedral structures exhibit a criticality characteristic in between the other two types of topologies, with \#1 walls exhibiting mostly dominant probabilities as most critical under the material strength variability ranges investigated in this study.
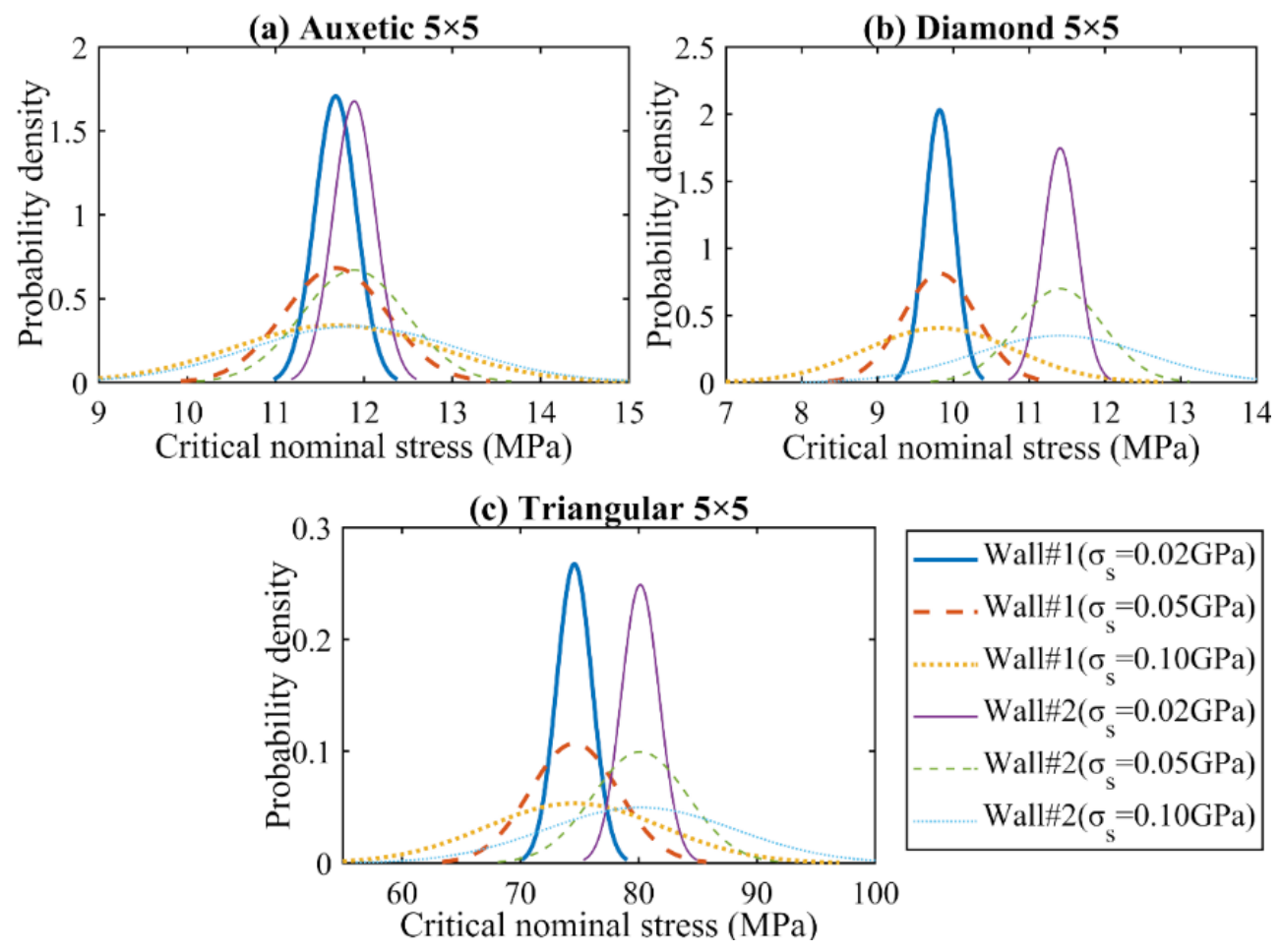

Fig. 5. 28 Probability density curves of critical nominal stress of Wall\#1 and Wall \#2 under different wall strength variability $\left(\sigma_{s}\right)$ for different structures

Therefore, the results shown in Table 5.6 will likely need to be revised for auxetic structures by including more walls into consideration in the extreme value analysis. For example, including both walls \#1 and \#2 would correspond to a problem that determines the minimum value of 8 independent normal distribution functions that follow 2 distribution characteristics. Fig. 5.29 illustrates the improvement of prediction accuracy of 
the average strength reduction of the structures as more critical wall indices are included into the analysis. As shown in Fig. 5.29(a), for the auxetic structure, even at moderate material strength variability level of $\sigma_{s}=0.05 G P a$, there is a significant improvement of prediction accuracy $(5 \% \rightarrow 6 \%)$ as the included criticality indices increases from 1 to 2 , and the prediction eventually converges to $\sim 8 \%$ when the included criticality indices increases to 7. For the octahedral structures, significant improvement is only gained for the case with high level of material strength variability $\left(\sigma_{s}=0.10 G P a\right)$, with prediction gradually converges from $\sim 10 \%$ to $14 \%$ as the included criticality indices increases from 1 to 7 . For the BCC structures, the increase of the included criticality indices does not alter the accuracy of the predictions, indicating the high criticality of the wall \#1 within the structures.

Another design implication of the observations from Fig. 5.29 lays within the balance between accuracy and efficiency. For real-world cellular structure designs, the number of cellular wall/strut elements could become computationally expensive even with the analytical modeling approach, and therefore it would benefit the design efficiency if the number of wall/strut elements that need to be analyzed for property variability evaluation can be reduced. For the diamond structure investigated in this study, for example, the conclusion clearly indicates that only the 4 most critical walls at the center of the structure need to be analyzed for accurate estimation of the structural strength variability. On the other hand, the characteristic of auxetic structure to minimize local stress concentration effects can become a challenge in the design process, as full-scale analysis might become necessary for adequate structural property predictions. 

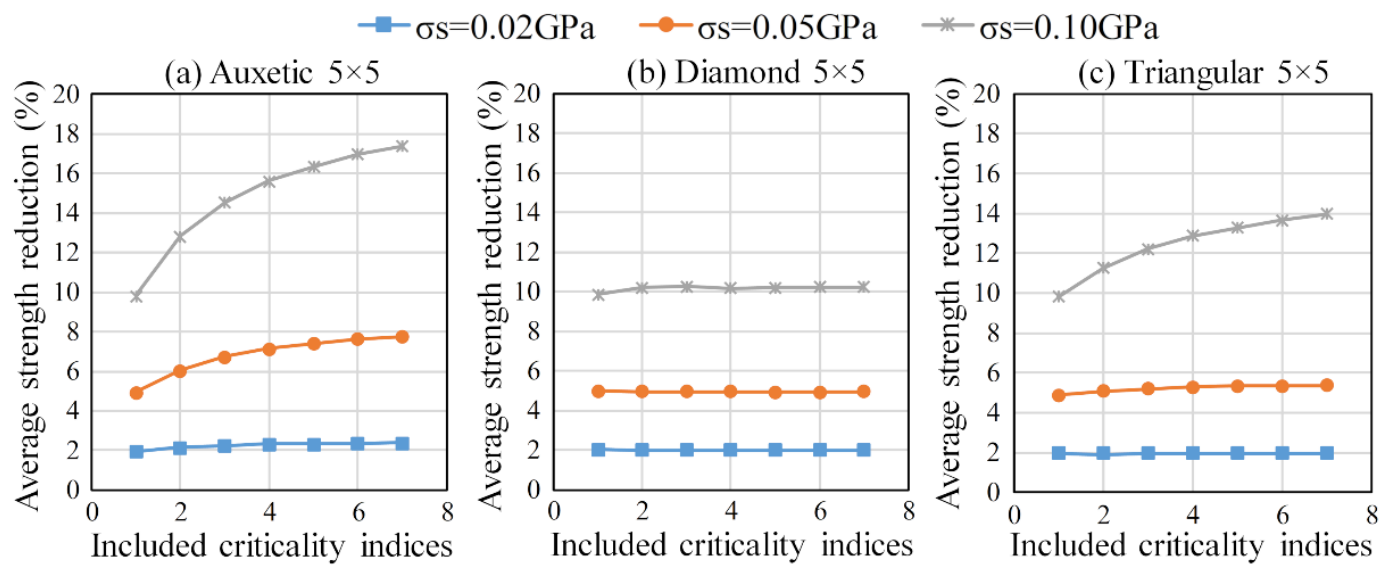

Fig. 5.29 The relationship between the included criticality indices and the average strength reduction

For the different failure patterns caused by the different level of material property variability, it can be explained with the help of the failure initiations. Based on the results shown in Section 5.3.1 and Section 5.3.2, it can be concluded that the wall strength variability has more significant effect on the mechanical properties of the $2 \mathrm{D}$ cellular structures compared to the wall modulus variability. Therefore, the effect of wall strength variability will be explored here. To elucidate the effect of cell wall strength variability, the initial failure locations of 50 individual runs for the three types of cellular structures at different levels of wall strength variabilities were marked and overlaid within a single structural graph for the $5 \times 5$ patterns, with the results shown in Fig. 5.30 . It can be seen that for different structures, the locations of critical walls follow specific distribution patterns, which could gradually vary with varying cell wall strength variabilities. With both the auxetic and triangular structures, as the wall strength variability increases, the locations of the critical walls gradually transition towards the center of the parts. Considering that for both types of structures the most critical walls for the cases with prefect material are located at the corners of the structures, it could be reasonably concluded that the center regions of 
these structures are the next critical locations, which could become more critical as the wall strength variability becomes significant. On the other hand, for the diamond structure, it appears that the structural effect is highly dominating, which leads to highly concentrated failure initiation site. As a result, even with relatively significant material strength variability, the failure pattern is still consistent.

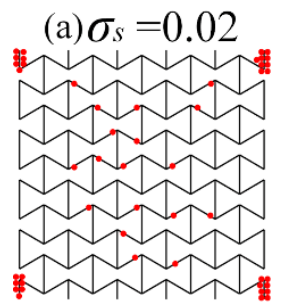

(d) $\sigma_{s}=0.02$

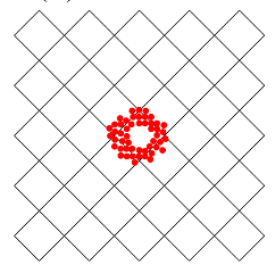

(g) $\sigma_{s}=0.02$

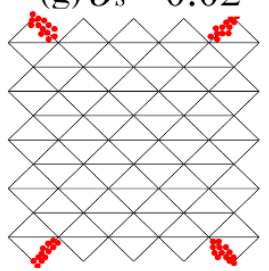

(b) $\sigma_{s}=0.05$

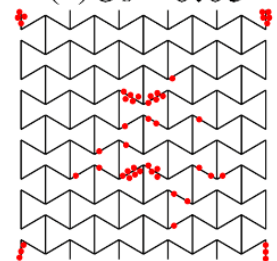

(e) $\sigma_{s}=0.05$

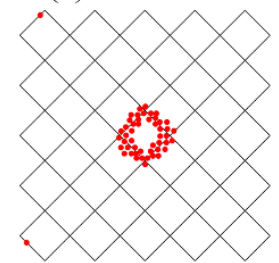

(h) $\sigma_{s}=0.05$

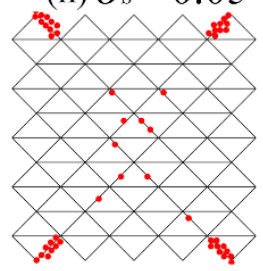

(c) $\sigma_{s}=0.10$

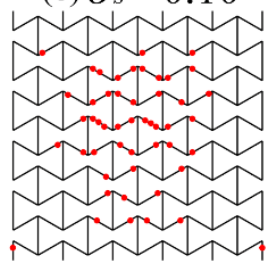

(f) $\sigma_{s}=0.10$

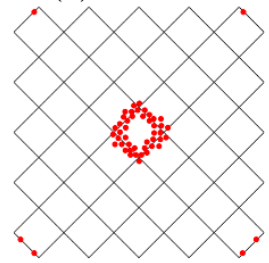

(i) $\sigma_{s}=0.10$

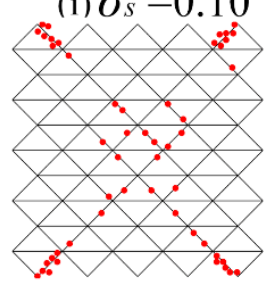

Fig. 5.30 The failure initiation distribution of different cellular types under different wall strength variability levels: $(\mathrm{a}, \mathrm{b}, \mathrm{c})$ auxetic structures; $(\mathrm{d}, \mathrm{e}, \mathrm{f})$ diamond structures; $(\mathrm{g}, \mathrm{h}, \mathrm{i})$ triangular structures

\subsection{Experimental verification}

\subsubsection{Sample design and fabrication}

A limited experimental verification was carried out to evaluate the accuracy of the analytical models. Thin wall and cellular structures made of Ti6Al4V (LPW, water 
atomized) using EOSINT M270 system. The process parameter setting closely followed the default process setting of M270 for support structure, i.e. laser power of $80 \mathrm{~W}$ and hatch scanning speed of $400 \mathrm{~mm} / \mathrm{s}$. In addition, the hatch spacing and layer thickness were set as $100 \mu \mathrm{m}$ and $30 \mu \mathrm{m}$, respectively. Other parameters were kept consistent to the default support structure parameter setting. No additional effort was made to optimize the process parameters, as it was irrelevant to the intended focus of this work. Thin wall tensile coupons with wall thickness of $0.5 \mathrm{~mm}$ was designs to obtain the baseline material property variability, and the cellular structure geometrical parameters were arbitrarily selected. For all the designs, the thickness in the $\mathrm{z}$ direction was arbitrarily set as $8 \mathrm{~mm}$, and the other geometrical design parameters are shown in Table 5.7. 10 tensile coupon samples and 5 samples of each cellular structure designs were fabricated, all in one batch in order to reduce the in-batch variability. All the thin walls were oriented along the same direction to ensure consistency. The samples on the substrate are shown in Fig. 5.31(a).

Table 5. 7 Design parameters of each fabricated cellular structure

\begin{tabular}{ccccccc}
\hline Structure & $\begin{array}{c}\text { Opening } \\
\text { angle }\end{array}$ & $\begin{array}{c}\text { Wall } \\
\text { length }\end{array}$ & $\begin{array}{c}\text { Wall } \\
\text { length }\end{array}$ & $\begin{array}{c}\text { Wall } \\
\text { thickness } \\
\left({ }^{\circ}\right)\end{array}$ & $\begin{array}{c}\text { Pattern } \\
\text { size }\end{array}$ & $\begin{array}{c}\text { Relative } \\
\text { density }\end{array}$ \\
\hline Auxetic & 60 & 2.89 & 1.98 & 0.5 & $5 \times 5$ & 0.362 \\
Diamond & 90 & 3.54 & - & 0.5 & $5 \times 5$ & 0.265 \\
Triangular & 90 & 3.54 & - & 0.5 & $5 \times 5$ & 0.411 \\
\hline
\end{tabular}

Tensile testing was carried out with the thin wall tensile coupons using an Instron 5569A (50kN load cell) testing system at a constant strain rate of $0.3 \mathrm{~mm} / \mathrm{min}$ for all the samples. Fig. 5.31b shows the strain-stress curves of the 10 thin wall tensile coupons. The 
cell wall strength was found to be $992.02 \mathrm{MPa}$ with a standard deviation of 92.85MPa. The cell wall modulus was found to be $111.75 \mathrm{GPa}$ with a standard deviation of $6.19 \mathrm{GPa}$.

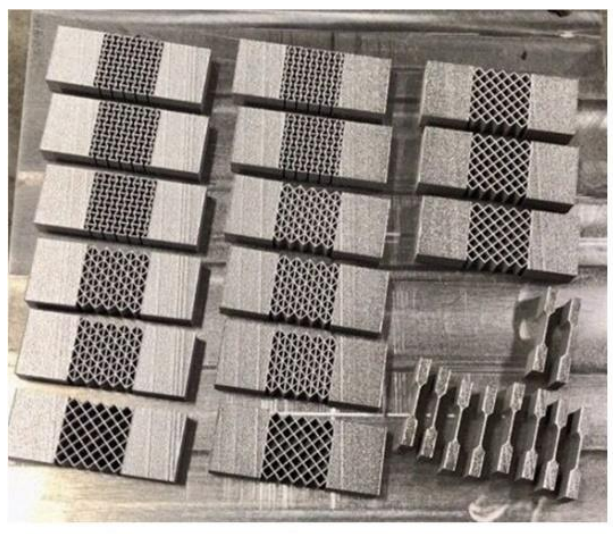

(a)

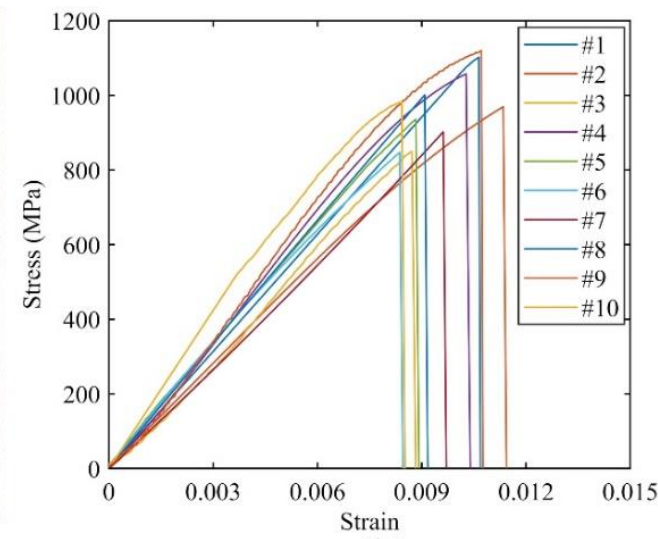

(b)

Fig. 5.31 Experimental verification using Ti6A14V samples fabricated via EOSINT M270: (a). Fabricated samples; (b). Stress-strain curves from tensile coupons

\subsubsection{Mechanical properties of cellular structures}

Fig. 5.32 exhibits the strain-stress curves of three cellular structures. Since the purpose of the study is to investigate the effect of the material property variability on the cellular structure property heterogeneity, the sample numbers are not labeled in the strainstress curves. As well-established for cellular structures under tensile loading, all tested structures initially exhibited an approximately elastic-catastrophic failure characteristic typical to the brittle materials with maximum stress failure mode that is adopted in this study. The figure shows that the cellular structures exhibited a progressive failure process similar to that in traditional honeycomb materials. Both auxetic and diamond structures exhibit obvious saw tooth-like stress-strain patterns with multiple stress peaks during the tensile failure process, with critical stress levels gradually decrease. As each stress peak corresponds to the fracture of one or more walls, multiple peaks indicate that the fracture is gradual. Therefore, for the bending-dominated auxetic and diamond structures, after one 
or multiple walls fail at each step, the remaining structures still exhibit some structural integrity and strength for further load application. For the triangular structures shown in Fig. 5.32(c), there is only one stress peak, which indicated that the stretching dominated triangular structures have a catastrophic failure. It is worth noting that even though both auxetic and diamond structures exhibit a decrease of the peak stresses during the failure, auxetic structures show a more dramatic decrease compared with diamond structures especially after the first peak stress.

(a)

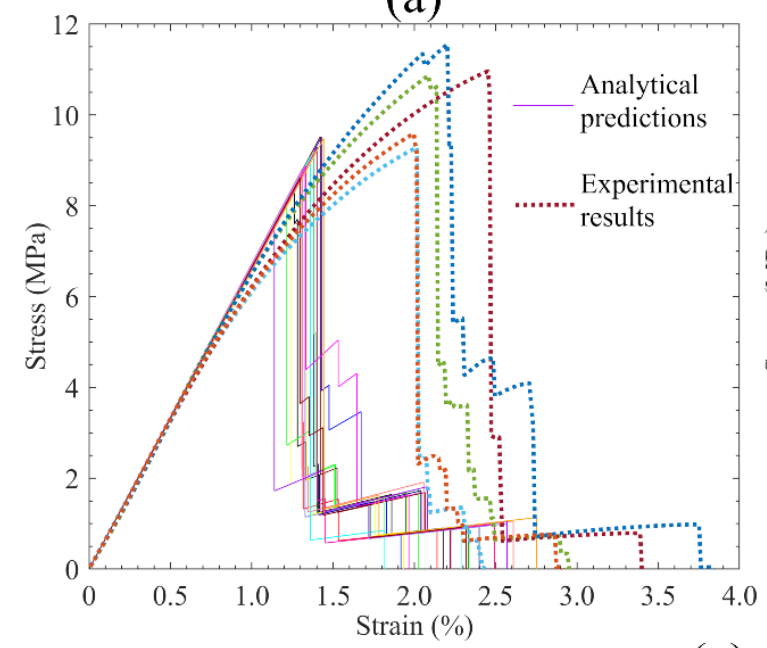

(b)

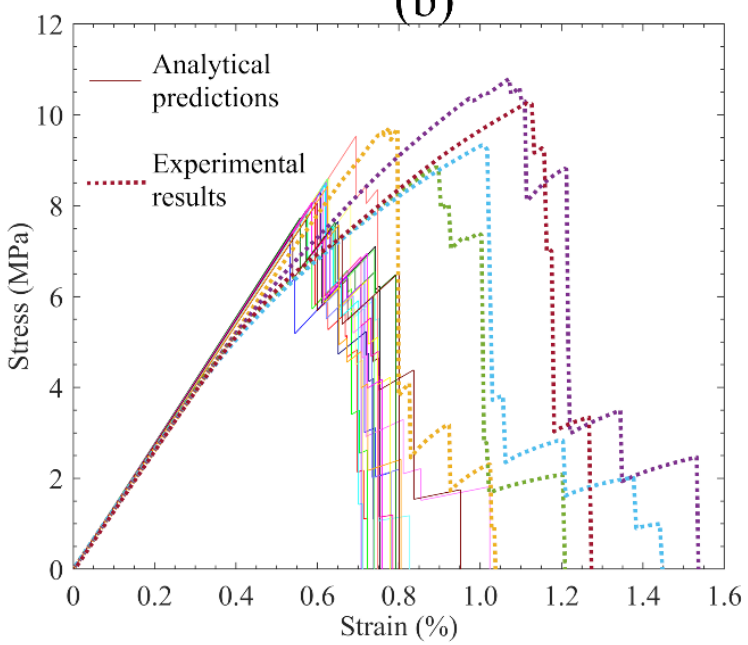

(c)

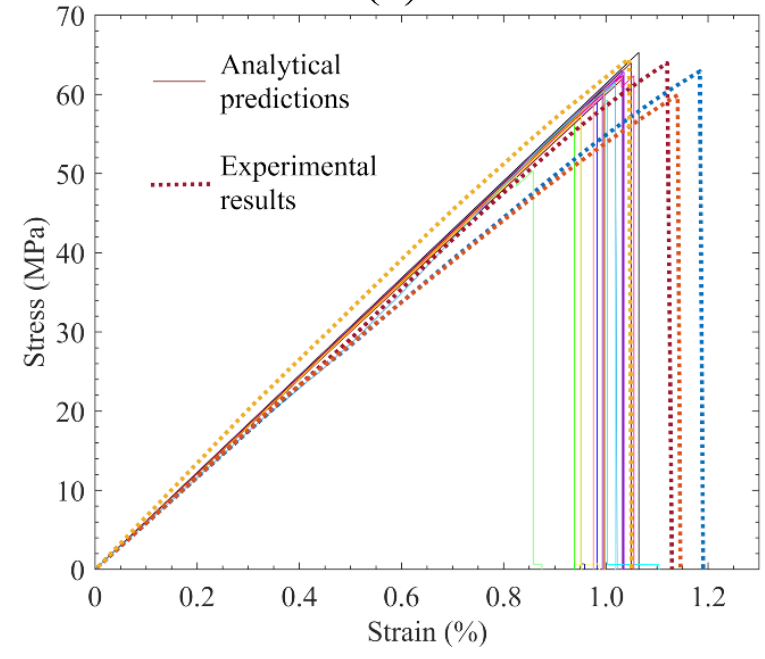

Fig. 5. 32 Strain-stress curves of (a)auxetic structures, (b) diamond structure; and (c) triangular structures 
Table 5.8 shows the comparison of the experimental results and the analytical predictions based on the experimentally established material variability. Overall, the predicted average strength and strength variability for all the structures are slightly lower than the experimental results. The discrepancy was attributed mainly to the plasticity of the material, which was not accounted for in the model but contribute to the load capacity of the structures. In addition, the plasticity of the material also tends to be a significant source of property variability. On the other hand, the predictions of the elastic modulus variabilities of the structures generally agree well with the experimental results. Another potential source or error could come from the data acquisition source for the strain, which was obtained from the displacement of the crosshead of the tensile testing system. As we discussed in Section 2.3, without the use of an extensometer, the estimation of elastic modulus with high-rigidity samples might be less accurate, which also appears to contribute to some of the lack of agreement of results with the elastic modulus of the triangular structures. Considering the comparison result that strain obtained from the DIC was around $70 \%$ of the strain obtained from the crosshead, we can see that using more accurate strain measurement method could result in a better agreement between the predicted and experimental results.

For the energy absorption results shown in Table 5.8, the predicted energy absorptions are obviously smaller than the experimental results. These discrepancies could be attributed mainly to the small portion of the plastic stage after elastic-linear stage of the material (shown in Fig. 5.31(b)), which was not accounted for in the model but contribute to a higher load capacity of the structures. When calculating the cellular tensile strength, only the first and maximum peak was utilized, which results in a small difference between 
the predicted and experimental strength. However, for the evaluation of the energy absorption, the deformation during the entire failure was included. The accumulation of energy absorption caused by the small plastic deformation of each failure step finally leads to larger difference between the predicted and experimental values. On the other hand, the predictions of the elastic modulus of the structures generally agree well with the experimental results. Nevertheless, from the failure characteristics based on the shape of the stress-strain curves, it is feasible to assume that the predicted results are reliable and can be used to make useful predictions.

Table 5.8 Analytical predictions and experimental results of the mechanical properties of different designs

\begin{tabular}{cccc}
\hline \multirow{2}{*}{ Cellular design } & Strength & Modulus & Energy absorption \\
\hline Auxetic & $8.867 \pm 0.527$ & $664.45 \pm 3.63$ & $71.79 \pm 5.80$ \\
Diamond & $8.200 \pm 0.483$ & $1368.82 \pm 9.58$ & $34.21 \pm 2.46$ \\
Triangular & $59.09 \pm 3.502$ & $6068.73 \pm 35.16$ & $291.89 \pm 28.97$ \\
\hline \multicolumn{4}{c}{ Experimental results } \\
Cellular design & Strength & Modulus & Energy absorption \\
\hline Auxetic & $10.462 \pm 0.974$ & $658.54 \pm 3.74$ & $146.77 \pm 28.11$ \\
Diamond & $9.788 \pm 0.754$ & $1316.32 \pm 8.05$ & $71.25 \pm 11.66$ \\
Triangular & $62.828 \pm 1.90$ & $5824.76 \pm 37.57$ & $372.94 \pm 39.67$ \\
\hline
\end{tabular}

5.4.3 Failure response of cellular structures

Fig. 5.33 illustrates the failure patterns of cellular structures obtained by experiments and analytical model, which are coincident with each other respectively. To exploring the effect of thin wall mechanical property variability on the cellular failure 
pattern variability, five samples were tested, and twenty analytical iterations were also generated to provide a stochastic result.

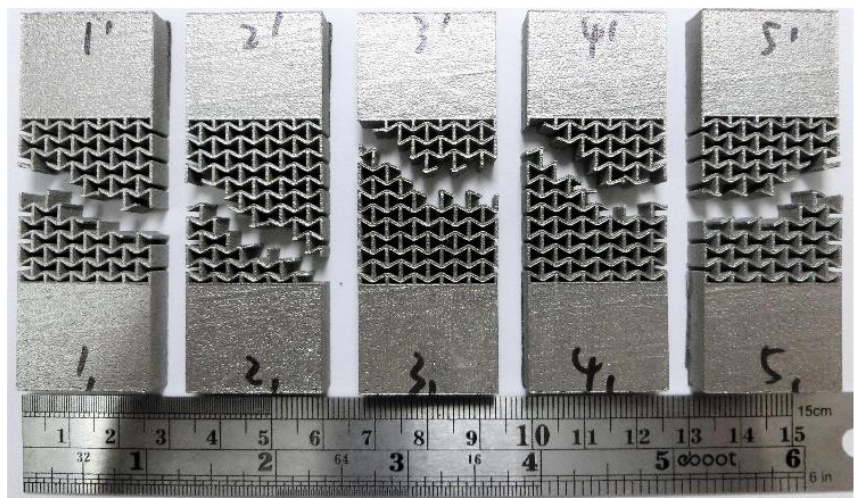

(a)

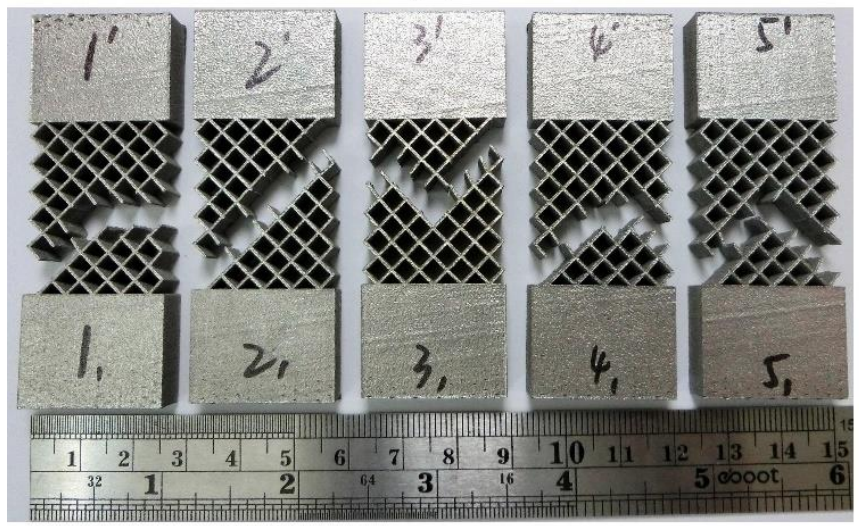

(c)

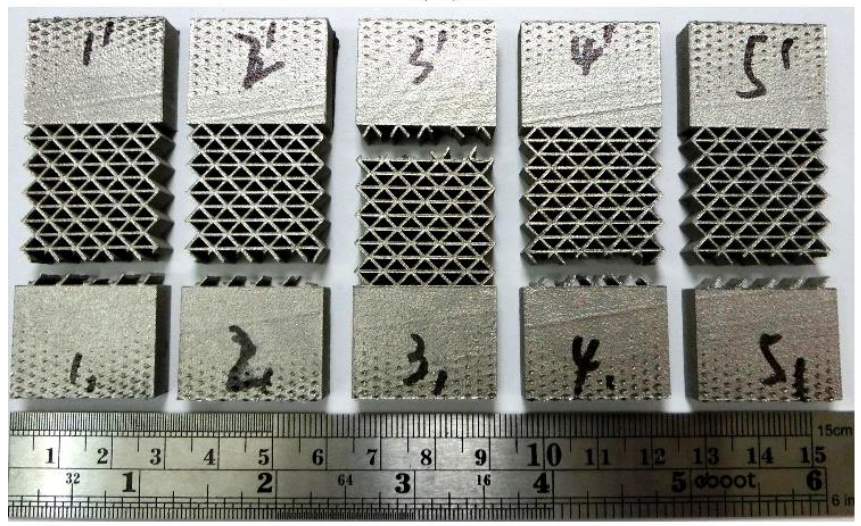

(e)

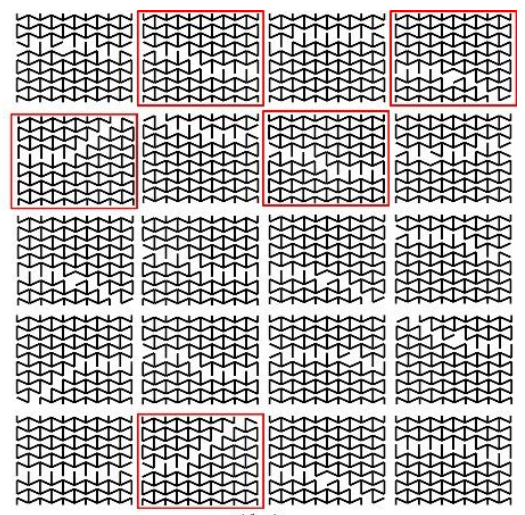

(b)

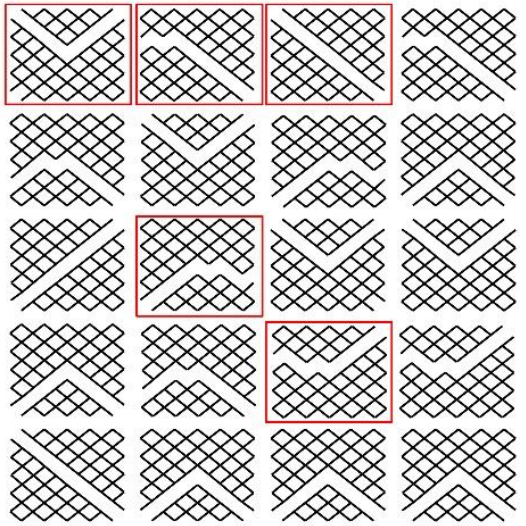

(d)

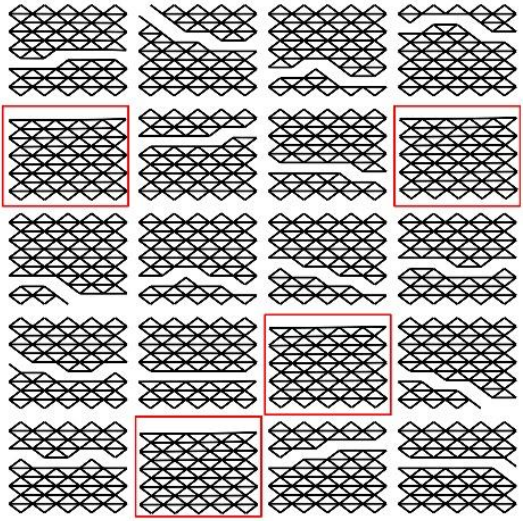

(f)

Fig. 5.33 Strain-stress curves of (a)auxetic structures, (b) diamond structure; and (c) triangular structures 
For the as-built auxetic structures, they mainly exhibited two typical failure patterns, shown in Fig. 5.33(a). Sample \#1 and \#2 showed a failure path located in the internal structure, while sample \#3, \#4 and \#5 have a failure path which transit from the corner to the internal of the structures, which matches well with the analytical predictions shown in Fig. 5.33(b). Based on stochastic result of the twenty random predictions, it can be seen that half of the samples show an internal fracture path and half of the samples show a transition fracture from corner to center when considering the material property variability. Comparing with the perfect auxetic structures which have an internal failure pattern, the existence of the defect lead to an additional failure pattern. For the as-built diamond structures, there are three main failure patterns shown in Fig. 5.33(c) even though there are some tiny different branches. Sample \#1 and \#4 showed a diagonal shear then horizontal pattern, and the other three samples have an either diagonal or V-shape failure pattern. And these experimental observations also agree with the analytical predictions shown in Fig. 5.33(d). Also based on the stochastic result of Fig. 5.33(d), it can be summarized that 9/20 of the samples exhibit a V-shape failure pattern, and 3/20 of the samples have a diagonal pattern, while $8 / 20$ of the samples exhibit a diagonal shear then horizontal pattern. For the perfect diamond structure, it is commonly known that the failure pattern tends to be diagonal. The process-induced defects make the failure pattern less consistent for diamond structures. For the triangular structures shown in Fig. 5.33(e) and Fig. 5.33(f), we can see that the experimental results were very consistent. The failure path all located on the top or bottom layer. However, for the analytical predictions shown in Fig. 5.33(f), the failure patterns tend to be less consistent. Apart from the top or bottom fractures, there also exist some fractures which locate in the middle of the structures. This discrepancy can be 
explained through the difference between the analytical model and fabrication model. For the analytical model, the top and bottom nodes can be simply constrained by the same displacements in the loading direction so that the cellular structures can be treated that both ends are fixed. However, for the fabricated sample, two rigid plates (Fig. 5.31(b)) need to be designed to help apply the displacement loading. For the auxetic structures (shown in Fig. 5.33(a)), it is the vertical walls that attached to the plates, which is consistent to the analytical model. For the diamond and triangular structures, two inclined walls generate the top and bottom nodes. For the analytical model shown in Fig. 2.11, these nodes have no interaction with the plates. For the fabricated model shown in Fig. 5.34(b,c), small portion $(0.2 \mathrm{~mm})$ of overlap design is needed for applying displacement loadings. This may result some discrepancy between the analytical and experimental results. Based on the FEA simulation result shown in Fig. 5.35, it can be found that compared with the auxetic and diamond structures, the triangular structures have obvious higher stress concentration on the top and bottom nodes which is attached to two plates. This higher stress concentration may result more potential failure on the boundary layer, which lead to the experimental failure pattern shown in Fig. 5.33(e).
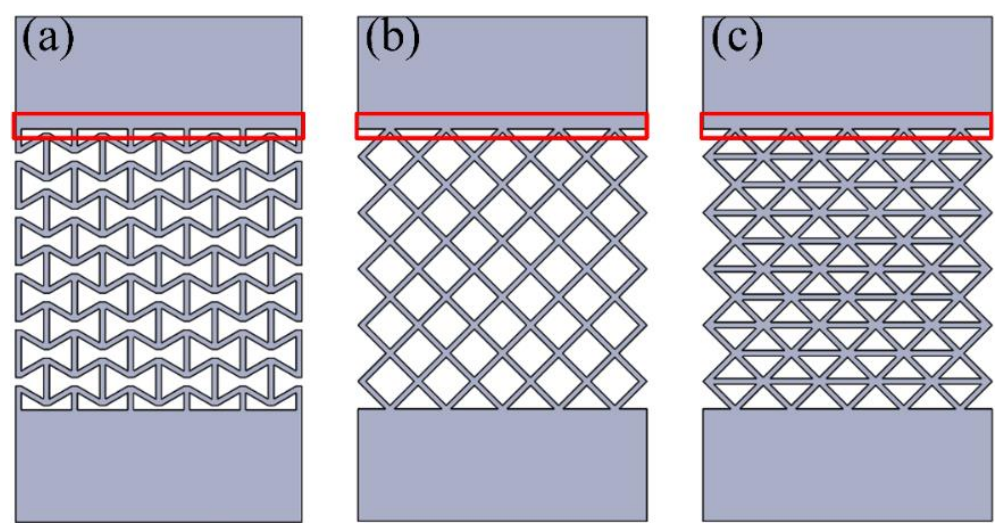

Fig. 5. 34 Boundary conditions of different structures: (a) auxetic structure; (b) diamond structure; (c) triangular structure 
(a)

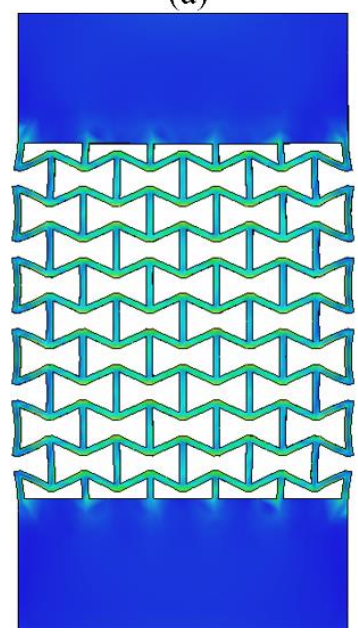

(b)

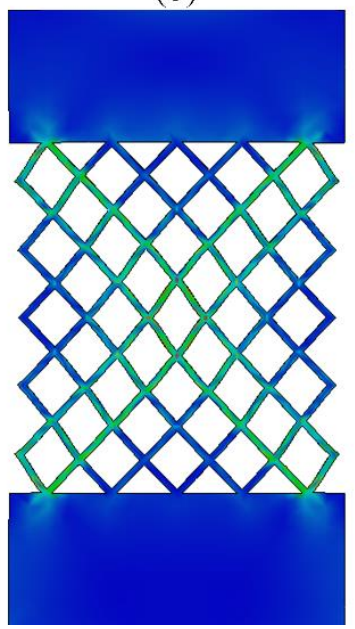

(c)

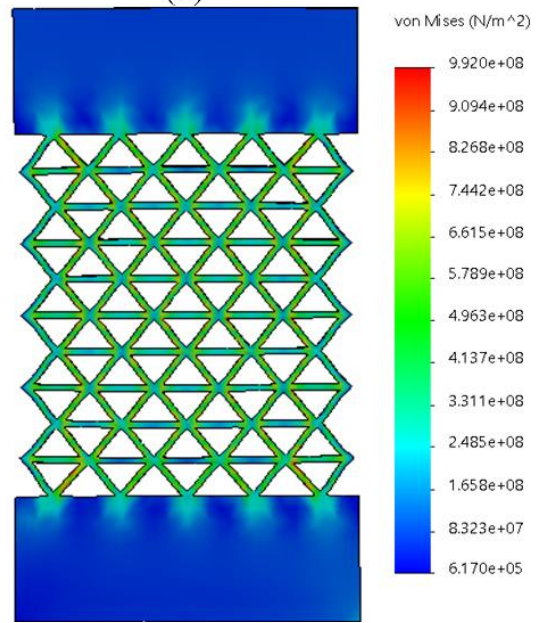

Fig. 5. 35 Simulation results for the perfect (a) auxetic, (b) diamond and (c) triangular structures

Combining with strain-stress curves shown section 5.4.2, it is found that the difference of the failure pattern for all structures does not significantly relate with the difference of their mechanical properties such as tensile strength, elastic modulus and energy absorption. However, for the cellular structures with defects, the failure patterns generally become various and difficult to predict.

\subsection{Conclusions}

In this section, an analytical model was established for the modeling of structural mechanical properties of cellular structures with imperfect materials at individual cellular element levels based on the model in Section 2. The effects of the local material property variability and structural pattern size on the mechanical properties of three $2 \mathrm{D}$ cellular structures, including the auxetic, diamond and triangular structures, were investigated with the help of the analytical models established. It was found that: 
1.The material property variability leads to a predictable decrease of the mechanical properties with the cellular structures. Intuitively, with higher level of material property variability, the structural properties tend to exhibit more significant decrease. In addition, the structural mechanical properties also exhibit increasing variability with increased material property variabilities, although generally at smaller levels. The mechanical properties of the structures are more sensitive to the material strength variability compared to the material modulus variability.

2.The structural pattern size only affects the averaged properties of the structures. The auxetic structures exhibit the smallest overall pattern size effects. In addition, the pattern size effects also significant deviate from the classic observations from previous literatures, which can be attributed to the boundary conditions imposed by finite-size structures.

3.Cellular structures with different topologies exhibit different characteristics to the material property variability levels. The triangular structures exhibit the highest sensitivity to material imperfection, while the auxetic structures exhibit the most significant overall strength reduction due to material imperfection.

4. The material property variability might also lead to multiple failure patterns to all three cellular structures. Besides, larger material property variability will result in more failure patterns.

5.The analytical model provides a reasonably good predictability to both the average and standard deviation of mechanical properties to the Ti6Al4V cellular structures fabricated via EOS M270 PBF system. Additional improvement of predictability could potentially be achieved via the incorporation of yield plasticity into the model. 


\section{CHAPTER VI}

\section{CONCLUSIONS}

\subsection{Conclusions}

In the current work, an analytical model based on stiffness matrix method for the mechanical property prediction for the cellular structure with finite unit cell patterns was established. The model was verified by the physical experiments for both mechanical properties and failure patterns, taking the advantage of the AM process. The model provides accurate predictions for the mechanical properties and failure response for cellular structures and could therefore be used as a convenient tool for design purposes.

It is widely recognized that the stretching-dominated structures are more advantageous for higher strength designs while the bending-dominated structures tend to exhibit more favorable compliance. In this work, the unit cell topology-size effect on the mechanical properties of several typical 2D stretching- and bending- dominated cellular structures were first systematically studied utilizing the established model. The conclusions of strength and modulus agree well with the existing knowledge grounds. The modulus and strength of a stretching-dominated cellular structure are much greater than those of a bending-dominated cellular structure with similar relative density. This makes stretchingdominated cellular structure attractive alternatives to bending-dominated structures for lightweight structure applications that need higher strength. For the bending-dominated structures, their progressive failure characteristic makes them more attractive as energyabsorbers since such application often requires a long and flat stress-strain response. 
However, it is worth nothing that the energy absorption capabilities of the 2D stretchingdominated structures still tend to be significantly higher than that of the 2D bendingdominated structures, which seems to contradict the argument that bending-dominated structures are always the first choice for energy-absorbers [25][26]. This could be explained by their failure characteristics and their initial strength. From the modeling analysis, the $2 \mathrm{D}$ bending-dominated structures with lower nodal connectivity exhibited relatively progressive crack propagation patterns, while the $2 \mathrm{D}$ stretching-dominated structures with higher nodal connectivity appear to exhibit rather catastrophic "brittle" fracture failure. Usually progressive failure response represents higher energy absorption ability. However, the smaller initial strength of bending-dominated structures still leads to small energy absorption ability compared to stretching-dominated structures. This provides a useful perspective for the energy absorber design of cellular structures that the choice of bending-or stretching- dominated mechanism is not the only factor to be considered. The initial strength of the structures also plays a significant role. Also, although the stretching dominated structures can absorb more energy, they also fail more catastrophically. On the other hand, the bending dominated structures fail progressively but absorb less energy.

The pure analytical analysis can easily provide a systematical investigation of the cellular structures with different topologies and geometries. However, for accurate predictions for the mechanical properties of the cellular structures, it is also critically important to identify and quantify about the fabrication issues that could affect the performance. Due to the complex geometry of cellular structures, AM processes, which offers the possibility to produce complex parts without the design constraints of traditional manufacturing routes, have been widely used for the fabrication of cellular structures. AM 
cellular structures are still inherently susceptible to certain levels of defects due to the complexity of the AM process. These defects will always result in the materials anisotropy and material property variability, which might reduce the accuracy of the predictions based on analytical models.

How the material anisotropy affects the mechanical properties of cellular structures is mainly depended on geometry of unit cell type and the baseline properties of materials, which can be established experimentally using single struts or walls with different build orientations. Among all the build orientations, vertical struts (perpendicular to the build plate) have the highest strength while horizontal struts (parallel to the build plate) have the lowest. This leads to the results that the cellular structures that contain vertical struts might have a higher relative strength compared to the cellular structures that contain horizontal struts. For the cellular structures with vertical/horizontal struts, changing the fabrication orientation can lead to significant mechanical property change. For the inclined struts, changing the build angle will lead to a significant change of its mechanical properties, which will further affect the mechanical properties of the whole cellular structures. Therefore, a baseline investigation of relationship between the strut build angle and its mechanical properties is necessary for providing guidelines for the optimal build orientation of cellular structures.

The other manufacturing factor, the material property variability, generally will lead to a predictable decrease of the mechanical properties and diverging failure patterns with the cellular structures. Higher level of material property variability always leads to more significant structural properties decrease for a given topology. Cellular structures with different topologies exhibit different sensitivities to the material property variability 
levels. For example, it is also worth noting that for some structures such as diamond structures, lower material variability does not result in a significant increase of the mechanical properties or more consistent failure responses. This might imply that it is necessary to balance the cost that might incur on improving the material quality (such as using fine powders, optimizing process parameters, or using post surface finishing process) versus the desirable mechanical quality control.

Combing all the factors discussed above, some basic design rules can be concluded and summarized below:

1. For the cellular applications which need higher strength and modulus, the stretching dominated cellular structures are recommend. Besides, during the fabrication, the optimal build orientation would be the orientation that can keep all the strut angle larger to the build plate considering from the material anisotropy aspect.

2. For the actual lightweight applications in which the fail-safe design is needed, bending-dominated structures, with a progressive failure mode, might possess some advantages. In addition, for a given cellular topology, changing the unit cell numbers can also make the design change from catastrophic failure to progressive failure, so that the rest of the structures can still retain partial loading capability after the initial failure. Additionally, intentionally increase material variability can also generate the progressive failure mode with some designs, which is counterintuitive but useful. This can be done through using relatively rough powder or suboptimal process parameters, which can also save the fabrication cost and optimization time. 
3. For the applications with enough design space in which consistent performance is the priority, increasing the unit cell number or decrease the material variability can both results in more consistent strength, modulus, and failure pattern.

Besides, to avoid the costs of fabrication and experimentation of cellular structures, analytical models which can precisely and efficiently predict the behavior of cellular structures are necessary. While FEA modeling method can be an optimal choice, managing the inherent tradeoff between the computational expense and accuracy of these models for the mechanical behavior prediction of cellular structures is still very important. The approach to model and characterize the manufacturing defects of the complex cellular structures which is adopted in this work also provide a more convenient and low-cost method. Regardless of the detailed defect information (such as rough surface and internal porosities), all this information is represented by the variability of the material modulus and strength anisotropy, which can reduce the time and cost of calculation and simulation without losing the accuracy. Therefore, it is entirely possible to employ this approach to realize the accurate and low-cost mechanical predictions for cellular structure, even for other manufacturing processes besides the AM processes.

Overall, this work does not only establish the analytical models for the cellular structures, but also investigates the process related issues of cellular structures when using the analytical model for the predictions. The analytical investigation, together with the experimental verification of different cellular designs provide guidelines for the future design of various cellular structures fabricated by various AM processes. 


\subsection{Future work and perspective}

Currently, the proposed analytical model for the cellular structure is based on the linear elastic material. The plasticity of material is still not modeled. This model might work well for the cellular structures made by brittle materials. For the materials with obvious plastic deformation, the accuracy of this model might not be desirable. In the future work, the plastic deformation after the linear elastic stage can be taken into consideration to provide a more comprehensive model for the plastic materials.

Additionally, the current study explored the size effect, material anisotropy and material property variability individually in different sections. However, in the real application of the cellular structures, these three issues might all exist in a single design. Even though this work offers some guideline to make a decision based on these three aspects, a more comprehensive model which incorporates all this information can be developed to provide a more accurate prediction for the cellular structure properties. 


\section{REFERENCES}

[1] Yang, L., Harrysson, O., West, H., \& Cormier, D. (2013). A comparison of bending properties for cellular core sandwich panels. Materials Sciences and Applications, 4(08), 471.

[2] Novak, N., Starčevič, L., Vesenjak, M., \& Ren, Z. (2019). Blast response study of the sandwich composite panels with 3D chiral auxetic core. Composite Structures, 210, $167-178$.

[3] Zhang, L., Chen, Y., He, R., Bai, X., Zhang, K., Ai, S., ... \& Fang, D. (2020). Bending behavior of lightweight $\mathrm{C} / \mathrm{SiC}$ pyramidal lattice core sandwich panels. International Journal of Mechanical Sciences, 171, 105409.

[4] Wu, X., Xiao, K., Yin, Q., Zhong, F., \& Huang, C. (2018). Experimental study on dynamic compressive behaviour of sandwich panel with shear thickening fluid filled pyramidal lattice truss core. International Journal of Mechanical Sciences, 138, 467475.

[5] Kooistra, G. W., Queheillalt, D. T., \& Wadley, H. N. (2008). Shear behavior of aluminum lattice truss sandwich panel structures. Materials Science and Engineering: A, 472(1-2), 242-250.

[6] Rehme, O., \& Emmelmann, C. (2006, February). Rapid manufacturing of lattice structures with selective laser melting. In Laser-based Micropackaging (Vol. 6107, p. 61070K). International Society for Optics and Photonics. 
[7] Bici, M., Brischetto, S., Campana, F., Ferro, C. G., Seclì, C., Varetti, S., ... \& Mazza, A. (2018). Development of a multifunctional panel for aerospace use through SLM additive manufacturing. Procedia CIRP, 67, 215-220.

[8] Scarpa, F., Ciffo, L. G., \& Yates, J. R. (2003). Dynamic properties of high structural integrity auxetic open cell foam. Smart Materials and Structures, 13(1), 49.

[9] Bai, L., Gong, C., Chen, X., Sun, Y., Xin, L., Pu, H., ... \& Luo, J. (2020). Mechanical properties and energy absorption capabilities of functionally graded lattice structures: experiments and simulations. International Journal of Mechanical Sciences, 105735.

[10] Chen, X., Ji, Q., Wei, J., Tan, H., Yu, J., Zhang, P., ... \& Kadic, M. (2020). Lightweight shell-lattice metamaterials for mechanical shock absorption. International Journal of Mechanical Sciences, 169, 105288.

[11] Evans, A. G., Hutchinson, J. W., \& Ashby, M. F. (1998). Multifunctionality of cellular metal systems. Progress in materials science, 43(3), 171-221.

[12] Qiu, L., Zou, H., Tang, D., Wen, D., Feng, Y., \& Zhang, X. (2018). Inhomogeneity in pore size appreciably lowering thermal conductivity for porous thermal insulators. Applied Thermal Engineering, 130, 1004-1011.

[13] Hutmacher, D. W. (2000). Scaffolds in tissue engineering bone and cartilage. Biomaterials, 21(24), 2529-2543.

[14] Karageorgiou, V., \& Kaplan, D. (2005). Porosity of 3D biomaterial scaffolds and osteogenesis. Biomaterials, 26(27), 5474-5491.

[15] Vilardell, A. M., Takezawa, A., du Plessis, A., Takata, N., Krakhmalev, P., Kobashi, M., ... \& Yadroitsev, I. (2019). Topology optimization and characterization of 
Ti6Al4V ELI cellular lattice structures by laser powder bed fusion for biomedical applications. Materials Science and Engineering: A, 766, 138330.

[16] Egan, P. F., Bauer, I., Shea, K., \& Ferguson, S. J. (2019). Mechanics of threedimensional printed lattices for biomedical devices. Journal of Mechanical Design, 141(3).

[17] Alabort, E., Barba, D., \& Reed, R. C. (2019). Design of metallic bone by additive manufacturing. Scripta Materialia, 164, 110-114.

[18] Free, B. R. 3D printing in healthcare market size and industry analysis report: global market forecast, market share, and trends, 2014-2022.

[19] Finnegan, K. A. (2007). Carbon fiber composite pyramidal lattice structures (Doctoral dissertation, University of Virginia).

[20] Chen Chu, Greg Graf, and David W. Rosen, (2008). Design for additive manufacturing of cellular structures, computer-aided design and applications, 5(5), 686-696.

[21] Ashby, M. F. (2000). Metal foams: a design guide. Butterworth-Heinemann. sintering of metals. Rapid Prototyp J 1995;1:26-36.

[22] Hussein, A. Y. (2013). The development of lightweight cellular structures for metal additive manufacturing.

[23] Kooistra, G. W., Deshpande, V. S., \& Wadley, H. N. (2004). Compressive behavior of age hardenable tetrahedral lattice truss structures made from aluminium. Acta Materialia, 52(14), 4229-4237.

[24] Gibson, L.J., Ashby, M.F., (1997).Cellular Solids, Structure and Properties, 2nd Edition, Cambridge, Cambridge University Press. 
[25] Deshpande V.S., Fleck N.A., (2001). Collapse of truss core sandwich beams in 3point bending, Int. J. Solids \& Structures, 38, p.6275.

[26] Deshpande, V. S., Ashby, M. F., \& Fleck, N. A. (2001). Foam topology: bending versus stretching dominated architectures. Acta materialia, 49(6), 1035-1040.

[27] Maxwell, J. C., Philosophical Magazine, 1864, 27, 294.

[28] Tao, W., \& Leu, M. C. (2016, August). Design of lattice structure for additive manufacturing. In 2016 International Symposium on Flexible Automation (ISFA) (pp. 325-332). IEEE.

[29] Brooks, W., Sutcliffe, C., Cantwell, W., Todd, J., \& Mines, R. (2005, August). Rapid design and manufacture of ultralight cellular materials. In 2005 International Solid Freeform Fabrication Symposium.

[30] Wettergreen, M. A., Bucklen, B. S., Starly, B., Yuksel, E., Sun, W., \& Liebschner, M. A. (2005). Creation of a unit block library of architectures for use in assembled scaffold engineering. Computer-Aided Design, 37(11), 1141-1149.

[31] Chen, Y. (2007). 3D texture mapping for rapid manufacturing. Computer-Aided Design and Applications, 4(6), 761-771.

[32] Jung, Y., Chu, K. T., \& Torquato, S. (2007). A variational level set approach for surface area minimization of triply-periodic surfaces. Journal of Computational Physics, 223(2), 711-730.

[33] Gandy, P. J., Bardhan, S., Mackay, A. L., \& Klinowski, J. (2001). Nodal surface approximations to the P, G, D and I-WP triply periodic minimal surfaces. Chemical physics letters, 336(3-4), 187-195. 
[34] Wang, Y. (2007). Periodic surface modeling for computer aided nano design. Computer-Aided Design, 39(3), 179-189.

[35] Kapfer, S. C., Hyde, S. T., Mecke, K., Arns, C. H., \& Schröder-Turk, G. E. (2011). Minimal surface scaffold designs for tissue engineering. Biomaterials, 32(29), 68756882.

[36] Rodrigues, H. C. (2006). Topology optimization of structures: applications in the simulation and design of cellular materials. In Computational Methods in Engineering \& Science (pp. 101-112). Springer, Berlin, Heidelberg.

[37] Nguyen, C. H. P., Kim, Y., \& Choi, Y. (2019). Design for additive manufacturing of functionally graded lattice structures: a design method with process induced anisotropy consideration. International Journal of Precision Engineering and Manufacturing-Green Technology, 1-17.

[38] García-Domínguez, A., Claver, J., \& Sebastián, M. A. (2019). Infill optimization for pieces obtained by 3D printing. Procedia Manufacturing, 41, 193-199.

[39] Brackett, D., Ashcroft, I., \& Hague, R. (2011, August). Topology optimization for additive manufacturing. In Proceedings of the solid freeform fabrication symposium, Austin, TX (Vol. 1, pp. 348-362).

[40] Zadpoor, A. A. (2019). Mechanical performance of additively manufactured metabiomaterials. Acta biomaterialia, 85, 41-59.

[41] Ashby, M. F. (2005). The properties of foams and lattices. Philosophical Transactions of the Royal Society A: Mathematical, Physical and Engineering Sciences, 364(1838), $15-30$. 
[42] Koehnen, P., Haase, C., Bueltmann, J., Ziegler, S., Schleifenbaum, J. H., \& Bleck, W. (2018). Mechanical properties and deformation behavior of additively manufactured lattice structures of stainless steel. Materials \& Design, 145, 205-217.

[43] Merkt, S., Hinke, C., Bültmann, J., Brandt, M., \& Xie, Y. M. (2015). Mechanical response of TiAl6V4 lattice structures manufactured by selective laser melting in quasistatic and dynamic compression tests. Journal of laser applications, 27(S1), S17006.

[44] Campoli, G., Borleffs, M. S., Yavari, S. A., Wauthle, R., Weinans, H., \& Zadpoor, A. A. (2013). Mechanical properties of open-cell metallic biomaterials manufactured using additive manufacturing. Materials \& Design, 49, 957-965.

[45] Ahmadi, S. M., Yavari, S. A., Wauthle, R., Pouran, B., Schrooten, J., Weinans, H., \& Zadpoor, A. A. (2015). Additively manufactured open-cell porous biomaterials made from six different space-filling unit cells: The mechanical and morphological properties. Materials, 8(4), 1871-1896.

[46] Sallica-Leva, E., Jardini, A. L., \& Fogagnolo, J. B. (2013). Microstructure and mechanical behavior of porous $\mathrm{Ti}-6 \mathrm{Al}-4 \mathrm{~V}$ parts obtained by selective laser melting. Journal of the mechanical behavior of biomedical materials, 26, 98-108.

[47] Esfahani, S. N., Andani, M. T., Moghaddam, N. S., Mirzaeifar, R., \& Elahinia, M. (2016). Independent tuning of stiffness and toughness of additively manufactured titanium-polymer composites: Simulation, fabrication, and experimental studies. Journal of Materials Processing Technology, 238, 22-29.

[48] Kadkhodapour, J., Montazerian, H., Darabi, A. C., Anaraki, A. P., Ahmadi, S. M., Zadpoor, A. A., \& Schmauder, S. (2015). Failure mechanisms of additively 
manufactured porous biomaterials: Effects of porosity and type of unit cell. Journal of the mechanical behavior of biomedical materials, 50, 180-191.

[49] Ahmadi, S. M., Campoli, G., Yavari, S. A., Sajadi, B., Wauthlé, R., Schrooten, J., ... \& Zadpoor, A. A. (2014). Mechanical behavior of regular open-cell porous biomaterials made of diamond lattice unit cells. Journal of the mechanical behavior of biomedical materials, 34, 106-115.

[50] Shi, X., Liao, W., Li, P., Zhang, C., Liu, T., Wang, C., \& Wu, J. (2020). Comparison of Compression Performance and Energy Absorption of Lattice Structures Fabricated by Selective Laser Melting. Advanced Engineering Materials, 2000453.

[51] Liu, X., Sekizawa, K., Suzuki, A., Takata, N., Kobashi, M., \& Yamada, T. (2020). Compressive Properties of Al-Si Alloy Lattice Structures with Three Different Unit Cells Fabricated via Laser Powder Bed Fusion. Materials, 13(13), 2902.

[52] Maskery, I., Aboulkhair, N. T., Aremu, A. O., Tuck, C. J., Ashcroft, I. A., Wildman, R. D., \& Hague, R. J. (2016). A mechanical property evaluation of graded density AlSi10-Mg lattice structures manufactured by selective laser melting. Materials Science and Engineering: A, 670, 264-274.

[53] Zargarian, A., Esfahanian, M., Kadkhodapour, J., Ziaei-Rad, S., \& Zamani, D. (2019). On the fatigue behavior of additive manufactured lattice structures. Theoretical and Applied Fracture Mechanics, 100, 225-232.

[54] Yavari, S. A., Ahmadi, S. M., Wauthle, R., Pouran, B., Schrooten, J., Weinans, H., \& Zadpoor, A. A. (2015). Relationship between unit cell type and porosity and the fatigue behavior of selective laser melted meta-biomaterials. Journal of the mechanical behavior of biomedical materials, 43, 91-100. 
[55] Balla, V. K., Bodhak, S., Bose, S., \& Bandyopadhyay, A. (2010). Porous tantalum structures for bone implants: fabrication, mechanical and in vitro biological properties. Acta biomaterialia, 6(8), 3349-3359.

[56] Ahmadi, S. M., Hedayati, R., Li, Y., Lietaert, K., Tümer, N., Fatemi, A., ... \& Zadpoor, A. A. (2018). Fatigue performance of additively manufactured meta-biomaterials: The effects of topology and material type. Acta biomaterialia, 65, 292-304.

[57] Yavari, S. A., Wauthlé, R., van der Stok, J., Riemslag, A. C., Janssen, M., Mulier, M., ... \& Zadpoor, A. A. (2013). Fatigue behavior of porous biomaterials manufactured using selective laser melting. Materials Science and Engineering: C, 33(8), 4849-4858.

[58] Hrabe, N. W., Heinl, P., Flinn, B., Körner, C., \& Bordia, R. K. (2011). Compressioncompression fatigue of selective electron beam melted cellular titanium (Ti-6Al-4V). Journal of Biomedical Materials Research Part B: Applied Biomaterials, 99(2), 313320.

[59] Li, S. J., Murr, L. E., Cheng, X. Y., Zhang, Z. B., Hao, Y. L., Yang, R., ... \& Wicker, R. B. (2012). Compression fatigue behavior of Ti-6Al-4V mesh arrays fabricated by electron beam melting. Acta Materialia, 60(3), 793-802.

[60] Aşı, E. E., \& Bor, Ş. (2015). Fatigue behavior of Ti-6Al-4V foams processed by magnesium space holder technique. Materials Science and Engineering: A, 621, 157165.

[61] Leuders, S., Thöne, M., Riemer, A., Niendorf, T., Tröster, T., Richard, H. A., \& Maier, H. J. (2013). On the mechanical behaviour of titanium alloy TiAl6V4 manufactured 
by selective laser melting: Fatigue resistance and crack growth performance. International Journal of Fatigue, 48, 300-307.

[62] Van Hooreweder, B., Apers, Y., Lietaert, K., \& Kruth, J. P. (2017). Improving the fatigue performance of porous metallic biomaterials produced by Selective Laser Melting. Acta biomaterialia, 47, 193-202.

[63] Wauthle, R., Vrancken, B., Beynaerts, B., Jorissen, K., Schrooten, J., Kruth, J. P., \& Van Humbeeck, J. (2015). Effects of build orientation and heat treatment on the microstructure and mechanical properties of selective laser melted Ti6Al4V lattice structures. Additive Manufacturing, 5, 77-84.

[64] Amirkhani, S., Bagheri, R., \& Yazdi, A. Z. (2012). Manipulating failure mechanism of rapid prototyped scaffolds by changing nodal connectivity and geometry of the pores. Journal of biomechanics, 45(16), 2866-2875.

[65] Geng, X., Lu, Y., Liu, C., Li, W., \& Yue, Z. (2019). Fracture characteristic analysis of cellular lattice structures under tensile load. International Journal of Solids and Structures, 163, 170-177.

[66] Fleck, N. A., \& Qiu, X. (2007). The damage tolerance of elastic-brittle, twodimensional isotropic lattices. Journal of the Mechanics and Physics of Solids, 55(3), $562-588$.

[67] Yang, Y., Shan, M., Zhao, L., Qi, D., \& Zhang, J. (2019). Multiple strut-deformation patterns based analytical elastic modulus of sandwich BCC lattices. Materials \& Design, 181, 107916.

[68] Gibson, L. J., \& Ashby, M. F. (1999). Cellular solids: structure and properties. Cambridge university press. 
[69] Zhang, L., Ferreira, J. M., Olhero, S., Courtois, L., Zhang, T., Maire, E., \& Rauhe, J. C. (2012). Modeling the mechanical properties of optimally processed cordieritemullite-alumina ceramic foams by X-ray computed tomography and finite element analysis. Acta Materialia, 60(10), 4235-4246.

[70] Pabst, W., Uhlířová, T., Gregorová, E., \& Wiegmann, A. (2018). Young's modulus and thermal conductivity of closed-cell, open-cell and inverse ceramic foams-modelbased predictions, cross-property predictions and numerical calculations. Journal of the European Ceramic Society, 38(6), 2570-2578.

[71] Wang, Y., Wang, J., \& Jia, P. (2011). Performance of forced convection heat transfer in porous media based on Gibson-Ashby constitutive model. Heat transfer engineering, 32(11-12), 1093-1098.

[72] Agbisit, R., Alavi, S., Cheng, E., Herald, T., \& Trater, A. (2007). Relationships between microstructure and mechanical properties of cellular cornstarch extrudates. Journal of Texture Studies, 38(2), 199-219.

[73] Yan, C., Hao, L., Hussein, A., Young, P., \& Raymont, D. (2014). Advanced lightweight 316L stainless steel cellular lattice structures fabricated via selective laser melting. Materials \& Design, 55, 533-541.

[74] Yakout, M., Elbestawi, M. A., \& Veldhuis, S. C. (2019). Density and mechanical properties in selective laser melting of Invar 36 and stainless steel 316L. Journal of Materials Processing Technology, 266, 397-420.

[75] McKown, S. S. S. S., Shen, Y., Brookes, W. K., Sutcliffe, C. J., Cantwell, W. J., Langdon, G. S., ... \& Theobald, M. D. (2008). The quasi-static and blast loading 
response of lattice structures. International Journal of Impact Engineering, 35(8), 795810.

[76] Li, B. Q., Wang, C. Y., \& Lu, X. (2013). Effect of pore structure on the compressive property of porous Ti produced by powder metallurgy technique. Materials \& Design, $50,613-619$.

[77] Kenesei, P., Kádár, C., Rajkovits, Z. S., \& Lendvai, J. (2004). The influence of cellsize distribution on the plastic deformation in metal foams. Scripta Materialia, 50(2), 295-300.

[78] Maconachie, T., Leary, M., Lozanovski, B., Zhang, X., Qian, M., Faruque, O., \& Brandt, M. (2019). SLM lattice structures: Properties, performance, applications and challenges. Materials \& Design, 183, 108137.

[79] Yang, L., Harrysson, O., West, H., \& Cormier, D. (2015). Mechanical properties of 3D re-entrant honeycomb auxetic structures realized via additive manufacturing. International Journal of Solids and Structures, 69, 475-490.

[80] Gümrük, R. E. C. E. P., \& Mines, R. A. W. (2013). Compressive behaviour of stainless steel micro-lattice structures. International Journal of Mechanical Sciences, 68, 125139.

[81] Zhang, S., Dilip, S., Yang, L., Miyanaji, H., \& Stucker, B. (2015). Property evaluation of metal cellular strut structures via powder bed fusion AM. In Proceedings of Solid Freeform Fabrication (SFF) Symposium, Austin, TX, USA.

[82] Bhate, D., Van Soest, J., Reeher, J., Patel, D., Gibson, D., Gerbasi, J., \& Finfrock, M. (2016). A validated methodology for predicting the mechanical behavior of ULTEM- 
9085 honeycomb structures manufactured by fused deposition modeling. In Solid Freeform Fabrication Symposium (pp. 2095-2106).

[83] Zhu, H. X., Hobdell, J. R., \& Windle, A. H. (2000). Effects of cell irregularity on the elastic properties of open-cell foams. Acta materialia, 48(20), 4893-4900.

[84] Zhu, H. X., Hobdell, J. R., \& Windle, A. H. (2001). Effects of cell irregularity on the elastic properties of 2D Voronoi honeycombs. Journal of the Mechanics and Physics of Solids, 49(4), 857-870.

[85] Zhou, J., Shrotriya, P., \& Soboyejo, W. O. (2004). On the deformation of aluminum lattice block structures: from struts to structures. Mechanics of materials, 36(8), 723737.

[86] Imbalzano, G., Tran, P., Ngo, T. D., \& Lee, P. V. (2016). A numerical study of auxetic composite panels under blast loadings. Composite Structures, 135, 339-352.

[87] Deshpande, V. S., Fleck, N. A., \& Ashby, M. F. (2001). Effective properties of the octet-truss lattice material. Journal of the Mechanics and Physics of Solids, 49(8), 1747-1769.

[88] Wan, H., Ohtaki, H., Kotosaka, S., \& Hu, G. (2004). A study of negative Poisson's ratios in auxetic honeycombs based on a large deflection model. European Journal of Mechanics-A/Solids, 23(1), 95-106.

[89] Yan, C., Hao, L., Hussein, A., Bubb, S. L., Young, P., \& Raymont, D. (2014). Evaluation of light-weight AlSi10Mg periodic cellular lattice structures fabricated via direct metal laser sintering. Journal of Materials Processing Technology, 214(4), 856864. 
[90] Robert, F. (1985). An isotropic three-dimensional structure with Poisson's ratio=- 1 . Journal of Elasticity, 15, 427-430.

[91] Sarker, A., Tran, N., Rifai, A., Elambasseril, J., Brandt, M., Williams, R., ... \& Fox, K. (2018). Angle defines attachment: Switching the biological response to titanium interfaces by modifying the inclination angle during selective laser melting. Materials \& Design, 154, 326-339.

[92] Cansizoglu, O., Harrysson, O., Cormier, D., West, H., \& Mahale, T. (2008). Properties of Ti-6Al-4V non-stochastic lattice structures fabricated via electron beam melting. Materials Science and Engineering: A, 492(1-2), 468-474.

[93] Suard, M., Martin, G., Lhuissier, P., Dendievel, R., Vignat, F., Blandin, J. J., \& Villeneuve, F. (2015). Mechanical equivalent diameter of single struts for the stiffness prediction of lattice structures produced by Electron Beam Melting. Additive Manufacturing, 8, 124-131.

[94] Isa, M. A., \& Lazoglu, I. (2019). Five-axis additive manufacturing of freeform models through buildup of transition layers. Journal of Manufacturing Systems, 50, 69-80.

[95] Gebhardt, A., Hötter, J. S., \& Ziebura, D. (2014, January). Impact of SLM build parameters on the surface quality. In RTejournal-Forum für Rapid Technologie (Vol. 2014, No. 1).

[96] Alsalla, H., Hao, L., \& Smith, C. (2016). Fracture toughness and tensile strength of 316L stainless steel cellular lattice structures manufactured using the selective laser melting technique. Materials Science and Engineering: A, 669, 1-6.

[97] Qiu, C., Yue, S., Adkins, N. J., Ward, M., Hassanin, H., Lee, P. D., ... \& Attallah, M. M. (2015). Influence of processing conditions on strut structure and compressive 
properties of lattice lattice structures fabricated by selective laser melting. Materials Science and Engineering: A, 628, 188-197.

[98] Weißmann, V., Drescher, P., Bader, R., Seitz, H., Hansmann, H., \& Laufer, N. (2017). Comparison of single Ti6Al4V struts made using selective laser melting and electron beam melting subject to part orientation. Metals, 7(3), 91.

[99] Weißmann, V., Bader, R., Hansmann, H., \& Laufer, N. (2016). Influence of the structural orientation on the mechanical properties of selective laser melted Ti6Al4V open-porous scaffolds. Materials \& Design, 95, 188-197.

[100] Yan, C., Hao, L., Hussein, A., Bubb, S. L., Young, P., \& Raymont, D. (2014). Evaluation of light-weight AlSi10Mg periodic lattice lattice structures fabricated via direct metal laser sintering. Journal of Materials Processing Technology, 214(4), 856864.

[101] Yan, C., Hao, L., Hussein, A., \& Raymont, D. (2012). Evaluations of lattice structures manufactured using selective laser melting. International Journal of Machine Tools and Manufacture, 62, 32-38.

[102] Alcisto, J., Enriquez, A., Garcia, H., Hinkson, S., Steelman, T., Silverman, E., ... \& Dorey, J. (2011). Tensile properties and microstructures of laser-formed Ti-6Al-4V. Journal of materials engineering and performance, 20(2), 203-212.

[103] Kasperovich, G., \& Hausmann, J. (2015). Improvement of fatigue resistance and ductility of TiAl6V4 processed by selective laser melting. Journal of Materials Processing Technology, 220, 202-214.

[104] Edwards, P., \& Ramulu, M. (2014). Fatigue performance evaluation of selective laser melted Ti-6Al-4V. Materials Science and Engineering: A, 598, 327-337. 
[105] Koike, M., Greer, P., Owen, K., Lilly, G., Murr, L. E., Gaytan, S. M., ... \& Okabe, T. (2011). Evaluation of titanium alloys fabricated using rapid prototyping technologies_electron beam melting and laser beam melting. Materials, 4(10), 17761792.

[106] Edwards, P., O'conner, A., \& Ramulu, M. (2013). Electron beam additive manufacturing of titanium components: properties and performance. Journal of Manufacturing Science and Engineering, 135(6).

[107] Dallago, M., Winiarski, B., Zanini, F., Carmignato, S., \& Benedetti, M. (2019). On the effect of geometrical imperfections and defects on the fatigue strength of cellular lattice structures additively manufactured via Selective Laser Melting. International Journal of Fatigue, 124, 348-360.

[108] Park, S. I., Rosen, D. W., \& Duty, C. E. (2014, August). Comparing mechanical and geometrical properties of lattice structure fabricated using Electron Beam Melting. In Proceedings of the 2014 Annual International Solid Freeform Fabrication Symposium-An Additive Manufacturing Conference, Austin, TX, USA (pp. 4-6).

[109] Park, S. I., Rosen, D. W., Choi, S. K., \& Duty, C. E. (2014). Effective mechanical properties of lattice material fabricated by material extrusion additive manufacturing. Additive Manufacturing, 1, 12-23.

[110] Yang, L., Harrysson, O., West, H., \& Cormier, D. (2013). Modeling of uniaxial compression in a 3D periodic re-entrant lattice structure. Journal of Materials Science, 48(4), 1413-1422.

[111] Liu, L., Kamm, P., García-Moreno, F., Banhart, J., \& Pasini, D. (2017). Elastic and failure response of imperfect three-dimensional metallic lattices: the role of geometric 
defects induced by Selective Laser Melting. Journal of the Mechanics and Physics of Solids, 107, 160-184.

[112] Lei, H., Li, C., Meng, J., Zhou, H., Liu, Y., Zhang, X., ... \& Fang, D. (2019). Evaluation of compressive properties of SLM-fabricated multi-layer lattice structures by experimental test and $\mu$-CT-based finite element analysis. Materials \& Design, 169, 107685.

[113] Lozanovski, B., Leary, M., Tran, P., Shidid, D., Qian, M., Choong, P., \& Brandt, M. (2019). Computational modelling of strut defects in SLM manufactured lattice structures. Materials \& Design, 171, 107671.

[114] Amani, Y., Dancette, S., Delroisse, P., Simar, A., \& Maire, E. (2018). Compression behavior of lattice structures produced by selective laser melting: X-ray tomography based experimental and finite element approaches. Acta Materialia, 159, 395-407.

[115] Ozdemir, Z., Hernandez-Nava, E., Tyas, A., Warren, J. A., Fay, S. D., Goodall, R., Todd, I., \& Askes, H. (2016). Energy absorption in lattice structures in dynamics: Experiments. International Journal of Impact Engineering, 89, 49-61.

[116] Ozdemir, Z., Tyas, A., Goodall, R., \& Askes, H. (2017). Energy absorption in lattice structures in dynamics: Nonlinear FE simulations. International Journal of Impact Engineering, 102, 1-15.

[117] Li, P., Wang, Z., Petrinic, N., \& Siviour, C. R. (2014). Deformation behaviour of stainless steel microlattice structures by selective laser melting. Materials Science and Engineering: A, 614, 116-121. 
[118] Maskery, I., Aboulkhair, N. T., Aremu, A. O., Tuck, C. J., \& Ashcroft, I. A. (2017). Compressive failure modes and energy absorption in additively manufactured double gyroid lattices. Additive Manufacturing, 16, 24-29.

[119] Yang, L. (2016). A study about size effects of 3D periodic cellular structures. In Proceedings of the 27th International Solid Freeform Fabrication (SFF) Symposium, Austin, TX.

[120] Ashby, M. F., \& Medalist, R. M. (1983). The mechanical properties of cellular solids. Metallurgical Transactions A, 14(9), 1755-1769.

[121] Maiti, S. K., Ashby, M. F., \& Gibson, L. J. (1984). Fracture toughness of brittle cellular solids. Scripta Metallurgica, 18(3), 213-217.

[122] O'Masta, M. R., Dong, L., St-Pierre, L., Wadley, H. N. G., \& Deshpande, V. S. (2017). The fracture toughness of octet-truss lattices. Journal of the Mechanics and Physics of Solids, 98, 271-289.

[123] Maimí, P., Turon, A., \& Trias, D. (2011). Crack propagation in quasi-brittle twodimensional isotropic lattices. Engineering Fracture Mechanics, 78(1), 60-70.

[124] Anderson, T. L. (2017). Fracture mechanics: fundamentals and applications. CRC press.

[125] Griffith, A. A. (1921). VI. The phenomena of rupture and flow in solids. Philosophical transactions of the royal society of london. Series A, containing papers of a mathematical or physical character, 221(582-593), 163-198.

[126] Huang, J. S., \& Gibson, L. J. (1991). Fracture toughness of brittle honeycombs. Acta metallurgica et materialia, 39(7), 1617-1626. 
[127] Huang, J. S., \& Gibson, L. J. (1991). Fracture toughness of brittle foams. Acta metallurgica et materialia, 39(7), 1627-1636.

[128] Hedayati, R., Hosseini-Toudeshky, H., Sadighi, M., Mohammadi-Aghdam, M., \& Zadpoor, A. A. (2016). Computational prediction of the fatigue behavior of additively manufactured porous metallic biomaterials. International journal of fatigue, 84, 67-79.

[129] Onck PR, Andrews EW, Gibson LJ. Size effects in ductile cellular solids. Part I: Modeling. Int J Mech Sci 2001;43(3):681-699. https://doi.org/10.1016/S00207403(00)00042-4.

[130] Brezny R, Green DJ. Characterization of edge effects in cellular materials. J Mater Sci 1990;25(11):4571-4578. https://doi.org/10.1007/BF01129908.

[131] Kiliçaslan C, Güden M, Odaci IK, Taşdemirci A. Experimental and numerical studies on the quasi-static and dynamic crushing responses of multi-layer trapezoidal aluminum corrugated sandwiches. Thin-Walled Struct 2014;78:70-78. https://doi.org/10.1016/j.tws.2014.01.017.

[132] Li C, Lei H, Liu Y, Zhang X, Xiong J, Zhou H, et al. Crushing behavior of multi-layer metal lattice panel fabricated by selective laser melting. Int J Mech Sci 2018; 145:389399. https://doi.org/10.1016/j.ijmecsci.2018.07.029.

[133] Yang L. Experimental-assisted design development for an octahedral cellular structure using additive manufacturing. Rapid Prototyp J 2015;21(2):168-176.

[134] Wang, A. J., \& McDowell, D. L. (2004). In-plane stiffness and yield strength of periodic metal honeycombs. J. Eng. Mater. Technol., 126(2), 137-156. 
[135] Wang, A. J., \& McDowell, D. L. (2005). Yield surfaces of various periodic metal honeycombs at intermediate relative density. International Journal of Plasticity, 21(2), 285-320.

[136] Huang, J. S., \& Lin, J. Y. (1996). Mixed-mode fracture of brittle lattice materials. Journal of materials science, 31(10), 2647-2652.

[137] Parthasarathy, J., Starly, B., Raman, S., \& Christensen, A. (2010). Mechanical evaluation of porous titanium (Ti6Al4V) structures with electron beam melting (EBM). Journal of the mechanical behavior of biomedical materials, 3(3), 249-259.

[138] Yang, L., Hsu, K., Baughman, B., Godfrey, D., Medina, F., Menon, M., \& Wiener, S. (2017). Additive Manufacturing of Metals: The Technology, Materials, Design and Production. Springer.

[139] Yang, L., Harrysson, O., Cormier, D., West, H., Gong, H., \& Stucker, B. (2015) Metal cellular structures with additive manufacturing: Design and fabrication. TMS Journal of Materials. 67, 608-615.

[140] List, F. A., Dehoff, R. R., Lowe, L. E., \& Sames, W. J. (2014). Properties of Inconel 625 mesh structures grown by electron beam additive manufacturing. Materials Science and Engineering: A, 615, 191-197.

[141] Lhuissier, P., de Formanoir, C., Martin, G., Dendievel, R., \& Godet, S. (2016). Geometrical control of lattice structures produced by EBM through chemical etching: Investigations at the scale of individual struts. Materials \& design, 110, 485-493.

[142] Vrána, R., Koutný, D., Paloušek, D., Pantělejev, L., Jaroš, J., Zikmund, T., \& Kaiser, J. (2018). Selective laser melting strategy for fabrication of thin struts usable in lattice structures. Materials, 11(9), 1763. 
[143] De Haan, L., \& Ferreira, A. (2007). Extreme value theory: an introduction. Springer Science \& Business Media. 


\section{CURRICULUM VITA}

\section{Yan $\mathrm{Wu}$}

Education

- Ph.D. candidate in Industrial Engineering, University of Louisville Expected November 2020 Louisville, KY, USA

- M.E. in Materials Processing Engineering, Huazhong University of Science \& Technology 2015 Wuhan, Hubei, China

- B.E. in Mechanical Design Manufacture and Automation, Wuhan University of Technology 2012 Wuhan, Hubei, China

Research Experience \& Projects

- University of Louisville | Design, modeling and simulation of cellular structures Louisville, KY, USA

Ph.D. Researcher |Advisor: Li Yang

Aug. 2015 - present

$>$ Proposed and built a theoretical model for the lightweight cellular structure for mechanical property prediction

$>$ Developed a Matlab program that could successfully predict the progressive failure of cellular structures

$>$ Verified the proposed theoretical model through both experiment and simulation methods

$>$ Evaluated their mechanical properties to establish a design guideline for potential future applications

- University of Louisville | Mechanical characterization of thin wall/strut structure

Louisville, KY, USA

Ph.D. Researcher |Advisor: Li Yang

Aug. 2017 - Aug. 2018

Applied experimental investigation to establish a preliminary understanding of the material characteristics of the Ti6Al4V cellular wall/struts using Powder Bed Fusion process under various geometry design conditions

Built a relationship between the mechanical properties and geometry parameters of thin 
wall/ struts to provide a design guideline for the cellular structures

- University of Louisville | Predicting quality-related geometric parameters using machine learning for additive manufacturing

Louisville, KY, USA

Ph.D. Researcher |Advisor: Li Yang

Aug. 2017 - Aug. 2019

$>$ Designed and fabricated a series of thin wall structures to build a database of the relationship between AM processing parameter and part density

$>$ Applied machine learning to successfully predict the quality-related geometric parameters

Research Interest

- Additive manufacturing processes and materials

- Structure-property relations of cellular structures: Modeling, simulation and experiments

- Failure mechanism of cellular structures

Technical Skills

- Lightweight structure design: Analytical modeling for periodic cellular structure such as BCC, Octet truss and Auxetic structures

- Additive manufacturing processes: Electron Beam Melting, Selective Laser Melting, Selective Laser Sintering

- Materials characterization: Optical Microscope, Scanning Electron Microscope, X-ray powder diffraction

- Computational skills: Finite Element Analysis (ANSYS, Solidworks), Matlab Programming, Maple

Honors and Awards

- Industrial Engineering Graduate Student Award

- 2018 BASF Design Contest Winner

- Graduate Teaching Assistant scholarship

- OPPEIN scholarship

- Distinguished Contributions to Research Award

- Outstanding Undergraduate

- Student Merit Award

Journal Publications 
- Wu, Y., \& Yang, L. (2020). The effect of unit cell size and topology on tensile failure behavior of 2D lattice structures. International Journal of Mechanical Sciences, 170, 105342.

- Wu, Y., Xue, P., Wei, Q., Huang, J., Shi, Y., \& Liu, J. (2015). An integrated forming process for manufacturing Ti6Al4V impeller with a functional coating layer using hot isostatic pressing. Materials and Manufacturing Processes, 30(6), 756-765.

- Wu, Y., Xue, P., Wei, Q., \& Shi, Y. (2015). Near-Net-Shaping Hot Isostatic Pressing of Ti6Al4V Alloys Monolithic Bladed Disks. RARE METAL MATERIALS AND ENGINEERING, 44(2), 360-364.

- Xue, P., Wu, Y., Huang, J., Wei, Q., \& Shi, Y. (2014). Rapidly and Effectively Forming Complex Parts with High Performance by the Compound Process of HIP and SLS/SLM. Electromachining \& Mould, 1, 014.

- Wang, M., Wu, Y., Wei, Q., \& Shi, Y. (2020). Thermal Fatigue Properties of H13 Hot-Work Tool Steels Processed by Selective Laser Melting. Metals, 10(1), 116.

- Wang, M., Li, W., Wu, Y., Li, S., Cai, C., Wen, S., ... \& Chen, Z. (2019). High-Temperature Properties and Microstructural Stability of the AISI H13 Hot-Work Tool Steel Processed by Selective Laser Melting. Metallurgical and Materials Transactions B, 50(1), 531-542.

- Cai, C., Song, B., Wei, Q., Wu, Y., Xue, P., \& Shi, Y. (2017). Effect of tooling material on the internal surface quality of Ti6Al4V parts fabricated by hot isostatic pressing. Metallurgical and Materials Transactions A, 48(1), 34-38.

Manuscripts in Preparation

- Wu, Y., \& Yang, L. Modeling and analysis of material anisotropy-topology effects of 3D cellular structures fabricated by powder bed fusion additive manufacturing. Under review

- Wu, Y., \& Yang, L. Modeling of the effect of local material imperfection to the structural mechanical property variability of 2D finite-size cellular structures. Under review

- Wu, Y., \& Yang, L. Modeling of Progressive Failure of Brittle Lattice Structures. To be submitted for publication in spring 2020.

Conference Papers

- Wu, Y., \& Yang, L. Size and Topology Effects on Fracture Behavior of Cellular Structures. Proceedings of the 2018 Solid Freeform Fabrication Symposium.

- Ewing, C., Wu, Y., \& Yang, L. Microstructural and Mechanical Characterization of Ti6Al4V Cellular Struts Fabricated by Electron Beam Powder Bed Fusion Additive Manufacturing. Proceedings of the 2018 Solid Freeform Fabrication Symposium.

- Dobson, S., Wu, Y., \& Yang, L. Material Characterization for Lightweight Thin Wall Structures Using Laser Powder Bed Fusion Additive Manufacturing. Proceedings of the 2018 Solid Freeform Fabrication Symposium.

- Orange, A., Wu, Y., \& Yang, L. An Investigation of the Fatigue Strength of Multiple Cellular Structures Fabricated by Electron Beam Powder Bed Fusion Additive Manufacturing Process. Proceedings of the 2018 Solid Freeform Fabrication Symposium.

- Wu, Y., \& Yang, L. Modeling of crack propagation in 2D brittle finite lattice structures assisted by additive manufacturing. Proceedings of the 2017 Solid Freeform Fabrication Symposium. 
- Yang, L., O’Neil, C., \& Wu, Y. The Use of Electropolishing Surface Treatment on In718 Parts Fabricated by Laser Powder Bed Fusion Process. Proceedings of the 2017 Solid Freeform Fabrication Symposium.

- Yang, L., Wu, Y., Lassell, A., \& Zhou, B. Electropolishing of Ti6Al4V parts fabricated by electron beam melting. Proceedings of the 2016 Solid Freeform Fabrication Symposium.

- Xue, P. J., Wu, Y., Huang, J., Wei, Q. S., Shi, Y. S., \& Yan, C. Z. (2014). Near net shape forming process of a titanium alloy component using hot isostatic pressing with graphite mould. In Applied Mechanics and Materials (Vol. 599, pp. 81-87). Trans Tech Publications.

\section{Conference Presentations}

- Wu, Y. Modelling for the Tensile Fracture Characteristic of Cellular Structures under Tensile Load with Size Effect. Proceedings of the 2019 Solid Freeform Fabrication Symposium.

- Wu, Y. Size and Topology Effects on Fracture Behavior of Cellular Structures. Proceedings of the 2018 Solid Freeform Fabrication Symposium.

- Wu, Y. Modeling of crack propagation in 2D brittle finite lattice structures assisted by additive manufacturing. Proceedings of the 2017 Solid Freeform Fabrication Symposium. 